\title{
Signal Processing Methods in Riemannian Geometry with Application to Drone Detection
}

\author{
by \\ Hossein M. Chahrour, M.Sc. \\ A dissertation submitted to the \\ Faculty of Graduate and Postdoctoral Affairs \\ in partial fulfillment of the requirements for the degree of
}

Doctor of Philosophy in Electrical Engineering

Ottawa-Carleton Institute for Electrical and Computer Engineering

Department of Systems and Computer Engineering

Carleton University

Ottawa, Ontario

Septmeber, 2021

(C) Copyright

Hossein M. Chahrour, 2021 


\section{Abstract}

The number of drones manufactured by many companies, such as DJI, Parrot, and 3D-Robotics, is always on the rise. Drones are widely used for commercial purposes, such as the delivery of goods, surveying and monitoring public places. On the other hand, drones can also be used to perform terrorist attacks or can be used to transport illegal drugs. Thus, a fast and reliable drone detection technique is very much needed to allow enough time for countermeasures in critical situations. Drones are considered complex targets which can range in size from $10 \mathrm{~m}^{2}$ to $0.01 \mathrm{~m}^{2}$ with symmetrical shape and fluctuating radar cross section (RCS), hence low signal-to-interference-plusnoise ratio (SINR). Current radar systems with classical signal processing techniques might fail to detect drones in low SINR environments with limited number of received snapshots. Multiple-input multiple-output (MIMO) radar systems with signal processing methods in Riemannian space can be exploited to improve the probability of drone detection, enhance the robustness of the direction of arrival estimation and improve the minimum variance distortionless response beamforming by estimating the interference-plus-noise covariance matrix in Riemannian space.

This dissertation utilizes uniform linear array (ULA) MIMO radar systems and proposes two Riemannian geometry-based constant false alarm rate (CFAR) detectors, a direction of arrival estimation technique based on Riemannian mean and distance, and interference-plus-noise covariance matrix estimation for beamforming in a Riemannian space. All proposed techniques exploit the regularized Burg algorithm (RBA) to convert each range bin into a Toeplitz Hermitian positive definite (THPD) matrix, which represents a point on the Riemannian manifold. Although Toeplitz structure is generated from ULA configurations, non linear array configurations would produce non-Toeplitz covariance matrices even if RBA guarantee Toeplitz structure. The proposed Riemannian-Brauer matrix (RBM) CFAR detector is based on the Riemannian distance between the Riemannian mean of the clutter-plus-noise Brauer bound and the THPD covariance matrices of the outliers. Also, the proposed 
angle-based hybrid-Brauer (ABHB) CFAR detector is based on the calculated angle on the Riemannian manifold between the Riemannian mean and median of the clutter-plus-noise Brauer bound and the THPD covariance matrix of the outliers. The direction of arrival estimation problem is formulated as a linear search optimization problem that searches for the minimum Riemannian distance between the Riemannian mean of all THPD covariance matrices residing on the manifold and the Hermitian positive definite (HPD) matrix for each of the steering vectors. The estimation of the interference-plus-noise covariance matrix is formulated as a linear combination of THPD covariance matrices where the weights of the linear combination operation are based on the Riemannian distance between the Riemannian mean and each THPD covariance matrix. The largest distance (potential target) will have zero weight and the smallest distance will have maximum weight. Simulations and real data analysis validate the robustness and performance of all techniques in low SINR and small sample size. 


\section{Acknowledgments}

First and foremost, I would to thank ALLAH, the most gracious, the most merciful and the most beneficent for all His blessings and generosity all the time. Second, I would like to thank my wife for her encouragement, support, patience and motivation. Also, I would like to thank all my family for their support, especially my daughter Reina and son Hassan.

I would like to express my deep and sincere gratitude to my supervisors, Professor Richard Dansereau and Professor Sreeraman Rajan, for their motivation, support, patience and funding. Also, a debt of gratitude is owed to Dr Bhashyam Balaji from Defence Research and Development Canada for his support and motivation.

Last but not the least, I would like to thank Defence Research and Development Canada and Natural Sciences and Engineering Research Council of Canada for their support and funding. 


\section{Contents}

Abstract $\quad$ ii

Acknowledgments $\quad$ iv

Table of Contents $\quad$ v

List of Tables $\quad$ ix

List of Figures $\quad$ x

Nomenclature $\quad$ xiv

1 Introduction 1

1.1 Drone Detection and Motivation . . . . . . . . . . . . . . 1

1.2 Problem Statement and Research Objective . . . . . . . . . . . 3

1.2.1 Hypothesis Statement . . . . . . . . . . . . 3

1.3 Dissertation Organization ................ 4

1.4 Contributions and Publications ............... . . 5

1.4.1 Matrix CFAR Detector . . . . . . . . . . . . 5

1.4.2 Direction of Arrival Estimation in Riemannian Space . . . . . 6

1.4.3 Covariance Matrix Estimation for Beamforming Applications . 6

2 Related Work and Fundamental Technology $\quad 8$

2.1 Drone Detection Techniques . . . . . . . . . . . . . . . . 8

2.2 Radar Technology . . . . . . . . . . . . . . . . . . . . 10

2.2.1 Multi-Antenna Radar Systems . . . . . . . . . . . . . . . . . . 12

2.2.2 Basic Radar Detection . . . . . . . . . . . . . . . . . 13

2.2.3 Constant False Alarm Rate Concept . . . . . . . . . . . . . 16 
2.3 Unmanned Aerial Vehicle . . . . . . . . . . . . . . . . . . . . 17

2.4 Regularized Burg Algorithm . . . . . . . . . . . . . . . . . . 19

2.5 Hermitian Positive Definite Matrices . . . . . . . . . . . . . 22

2.6 Differential Geometry and Manifolds . . . . . . . . . . . . . 23

2.6.1 Riemannian Manifold . . . . . . . . . . . . . . . . . . . 23

2.7 Eigenvalue Localization Techniques . . . . . . . . . . . . . 28

2.8 Conclusion . . . . . . . . . . . . . . . . . . . . . . . . 31

3 Matrix CFAR Detection in Riemannian Space 32

3.1 Cell-Averaging CFAR Detector _ . . . . . . . . . . . . 33

3.2 Signal Model and Detection Formulation . . . . . . . . . . . 36

3.3 Riemannian Distance Based Detector . . . . . . . . . . . . . 37

3.3 .1 RD-CFAR Detector . . . . . . . . . . . . . . . . 37

3.4 Kullback-Leibler Detector . . . . . . . . . . . . . . . . . . 39

3.5 Riemannian-Brauer Based Detectors . . . . . . . . . . . . . . 41

3.5.1 Motivation . . . . . . . . . . . . . . . . . . . 41

3.5.2 Brauer Cluster Bound . . . . . . . . . . . . . . . . 42

3.5.3 Riemannian-Brauer Matrix CFAR Detector . . . . . . . . 43

3.5.4 Angle Based Hybrid-Brauer CFAR Detector . . . . . . . . . 46

3.6 Simulations Results . . . . . . . . . . . . . . . . . . . 50

3.7 Real Data Analysis . . . . . . . . . . . . . . . . . . 60

3.8 Conclusion . . . . . . . . . . . . . . . . . . . . . . . . 69

4 Direction of Arrival Estimation in the Riemannian Space $\quad \mathbf{7 0}$

4.1 Uniform Linear Antenna Array and System Signal Model . . . . . . 71

4.2 Overview of DOA Estimation Techniques . . . . . . . . . . 72

4.3 Riemannian Geometry-based DOA Estimation . . . . . . . . 76

4.3 .1 Motivation . . . . . . . . . . . . . . . 76

$4.3 .2 \quad$ DOA Signal Model . . . . . . . . . . . . . . . . . . 77

4.3.3 DOA Estimation in the Riemannian Space . . . . . . . . 78

4.4 Simulation Results . . . . . . . . . . . . . . . . . . . . 79

4.5 Real Data Analysis . . . . . . . . . . . . . . . . . . . . . 91

4.6 Conclusion . . . . . . . . . . . . . . . . . . . . . . . . . 94 
5 Covariance Matrix Estimation for Beamforming Applications $\quad 95$

5.1 Array Signal Model and Adaptive Beamforming . . . . . . . . . 96

5.1 .1 Array Signal Model . . . . . . . . . . . . . . . . . 99 96

5.1 .2 MVDR Beamforming Algorithm . . . . . . . . . . . . . 98

5.2 Overview of Interference-plus-Noise Covariance Matrix Estimation

Techniques . . . . . . . . . . . . . . . . . . . . . . 999

5.3 Interference-plus-Noise Covariance Matrix Estimation in Riemannian

Space . . . . . . . . . . . . . . . . . . . . . 101

5.3 .1 Signal Model . . . . . . . . . . . . . . . . . . . . . . . . 102

5.3.2 Riemannian Geometry-based Interference-plus-Noise Covariance Matrix Estimation . . . . . . . . . . . . . . . . 103

5.4 Simulation Results . . . . . . . . . . . . . . . . . . . . . . 104

5.5 Real Data Analysis . . . . . . . . . . . . . . . . . . 110

5.6 Conclusion . . . . . . . . . . . . . . . . . . . . . . . . . . . . . 113

6 Conclusion and Future Work 114

6.1 Conclusion . . . . . . . . . . . . . . . . . . . . . . . . . 114

6.2 Limitations . . . . . . . . . . . . . . . . . . . 115

6.2.1 Covariance Matrix Structure . . . . . . . . . . . . . . . . 115

6.2.2 Antenna Array Configuration . . . . . . . . . . . . . 116

6.2 .3 Minimum Number of Range Bins . . . . . . . . . . . . . . 116

6.3 Future Work . . . . . . . . . . . . . . . . . . . . . 116

6.3.1 Antenna Configurations . . . . . . . . . . . . 116

6.3.2 Geometric Means and Medians . . . . . . . . . . . . . . 117

6.3.3 Cramér-Rao Lower Bound . . . . . . . . . . . . . . . . . . . . 117

6.3.4 Micro-Doppler Signature in Riemannian Space . . . . . . . . . 117

6.3.5 Target Detection and Classification with Manifold Learning . 117

$\begin{array}{ll}\text { List of References } & 119\end{array}$

Appendix A DOA Estimation Techniques $\quad 130$

A.1 Conventional DOA Estimation . . . . . . . . . . . . . . . . . 130

A.1.1 Delay and Sum Technique . . . . . . . . . . . . . . . . 130

A.1.2 Minimum Variance Distortionless Response . . . . . . . . . . . 132

A.2 Subspace Based DOA Estimation . . . . . . . . . . . . . . . 133 
A.2.1 MUSIC Algorithm . . . . . . . . . . . . . . . . 133

A.2.2 ESPRIT Algorithm . . . . . . . . . . . . . . . . . 135

A.3 Compressive Sensing and Weighted Spatial Filter DOA Techniques . 139

A.3.1 Compressive Sensing $\ell_{2,1}$ DOA estimation . . . . . . . . 139

A.3.2 Weighted Spatial Filter $\ell_{2,1}$ DOA Estimation . . . . . . . . 142

Appendix B Interference plus Noise Covariance matrix Estimation $\mathbf{1 4 5}$

B.1 Sample Covariance Matrix with Adaptive Diagonal Loading . . . . . 145

B.2 Shrinkage Algorithm for Covariance Matrix Estimation . . . . . . . 147

B.3 Subspace Based Interference-plus-Noise Covariance Matrix Estimation 149

B.4 Sparsity-Based Interference-plus-Noise Covariance Matrix Estimation 151 


\section{List of Tables}

3.1 AWR1642 automotive radar specifications. . . . . . . . . . . . . 60 


\section{List of Figures}

2.1 Block diagram of a typical conventional radar. . . . . . . . . . . . . . 10

2.2 Radar range and range resolution. . . . . . . . . . . . . . . . . 11

2.3 A typical 4 antenna element phased array radar transmitter. . . . . . 13

2.4 A typical $2 \times 2$ MIMO radar system. . . . . . . . . . . . . . . 14

2.5 ROC curves non-fluctuating target. . . . . . . . . . . . . 16

2.6 General CFAR processor. . . . . . . . . . . . . . . . 17

2.7 Yuneec Typhoon H drone. . . . . . . . . . . . . . . . . . . . . . 19

2.8 Tangent space of the manifold $\mathcal{M}$ at point $\mathbf{P}$ and the projection of $\mathbf{P}_{1}$ onto the tangent of point P. . . . . . . . . . . . . . . . . 24

2.9 Riemannian distance between two HPD covariance matrices $\mathbf{P}$ and $\mathbf{P}_{1}$ on the Riemannian manifold $\mathcal{M}$. . . . . . . . . . . . . . . . 26

2.10 Riemannian mean of the HPD covariance matrices on the Riemannian manifold M. . . . . . . . . . . . . . . . . . . . . . . . . . . . . . . . . 28

2.11 Typical Gershgorin discs for real eigenvalues. . . . . . . . . . . . 29

2.12 Gershgorin and Brauer discs for the maximum eigenvalue of covariance matrix R. . . . . . . . . . . . . . . . . . . . 30

3.1 Gaussian probability density functions of noise and target. . . . . . . 35

3.2 Cell averaging CFAR detector based on FFT. . . . . . . . . . . . . 36

3.3 Riemannian distance CFAR detector. . . . . . . . . . . . . 38

3.4 KLB based matrix CFAR detector. . . . . . . . . . . . . . . . 40

3.5 Example of Brauer cluster bound for clutter-plus-noise eigenvalues and maximum eigenvalues of potential targets THPD covariance matrices with their associated Brauer discs. . . . . . . . . . . . . . . . 44

3.6 RBM-CFAR detector. . . . . . . . . . . . . . . . . 45

3.7 Clutter-plus-noise THPD covariance matrices cluster bound and THPD covariance matrices of potential targets. . . . . . . . . . . . . 46

3.8 Geodesical triangle showing the angle at potential target on the manifold. 47 
3.9 ABHB-CFAR detector. . . . . . . . . . . . . . . . . . 49

3.10 Typical collocated $8 \times 8$ ULA antenna system with a drone target. . . 51

3.11 Typical collocated $8 \times 8$ RSLA antenna system with internal antennas spacing $[0.3 \lambda, 0.9 \lambda, 1.75 \lambda, 0.25 \lambda, 0.15 \lambda, 2.1 \lambda, 0.4 \lambda]$ and drone target. 52

3.12 Performance comparison of the CA-CFAR, RD-CFAR, KLB-CFAR, RMB-CFAR and ABHB-CFAR detectors with different $P_{f} \ldots \ldots .54$

3.13 Detection performance curves for original and scaled signal and noise.

3.14 Brauer bound for clutter-plus-noise and potential target at the $9^{\text {th }}$ range bin for ULA and RSLA configurations for $5 \mathrm{~dB}$ SNR. . . . . . 55

3.15 Detection performance of RBM and ABHB CFAR detectors for 16 snapshots pulse under $5 \mathrm{~dB}$ SNR. . . . . . . . . . . . . 56

3.16 Detection performance of CA-CFAR, RD-CFAR, KLB-CFAR, RBMCFAR and ABHB-CFAR detectors for pulse 1 for ULA. . . . . . . . .

3.17 Detection performance of CA-CFAR, RD-CFAR, KLB-CFAR, RBMCFAR and ABHB-CFAR detectors for pulse 1 for RSLA. . . . . . . .

3.18 Performance comparison of ABHB and RBM CFAR detectors for ULA, SPLA and RSLA for $P_{f}=10^{-4} \ldots \ldots \ldots \ldots \ldots$

3.19 Box plot for ABHB-CFAR and RBM-CFAR detectors for ULA, SPLA and RSLA configurations. . . . . . . . . . .

3.20 Brauer bound for clutter-plus-noise and potential targets at the $9^{\text {th }}$ and $10^{\text {th }}$ range bins for pulse $1 \ldots \ldots \ldots \ldots$

3.21 Brauer bound for clutter-plus-noise and potential targets at the $11^{\text {th }}$ and $12^{\text {th }}$ range bins for pulse $2 \ldots \ldots \ldots \ldots$

3.22 Brauer bound for clutter-plus-noise and potential targets at the $13^{\text {th }}$ and $14^{\text {th }}$ range bins for pulse $3 \ldots \ldots \ldots \ldots$

3.23 Detection performance of the RBM-CFAR detector for three consecutive pulses. . . . . . . . . . . . . . . .

3.24 Detection performance of the ABHB-CFAR detector for three consecutive pulses. . . . . . . . . . . . . . . .

3.25 Detection performance of the CA-CFAR, the RD-CFAR, the KLBCFAR, the RBM-CFAR and the ABHB-CFAR detectors for pulse 1 . .

3.26 Detection performance of the CA-CFAR, the RD-CFAR, the KLBCFAR, the RBM-CFAR and the ABHB-CFAR detectors under scaled SNR for pulse $1 \ldots \ldots \ldots \ldots \ldots \ldots \ldots \ldots \ldots$ 
3.27 Performance comparison of the CA-CFAR, the RD-CFAR, the KLBCFAR, the RBM-CFAR and the ABHB-CFAR detectors for different $P_{f} \ldots \ldots \ldots \ldots \ldots \ldots \ldots \ldots$

4.1 ULA structure with signal impinging from a particular direction. . . . 71

4.2 Spatial spectrum for RGDOA, DAS, MVDR and MUSIC for SNR=10

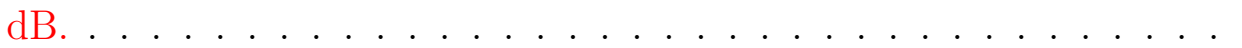

4.3 Spatial spectrum for RGDOA, DAS, MVDR and MUSIC for SNR=0

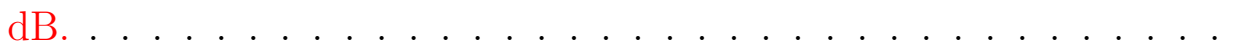

4.4 Spatial spectrum for RGDOA, DAS, MVDR and MUSIC for SNR=-5

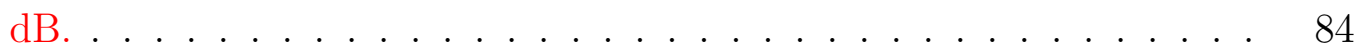

4.5 Spatial spectrum for RGDOA, DAS, MVDR and MUSIC for SNR=-10 dB............................... 85

4.6 DOA estimation with different sample sizes at $\mathrm{SNR}=10 \mathrm{~dB} \ldots \ldots$. . $\quad 86$

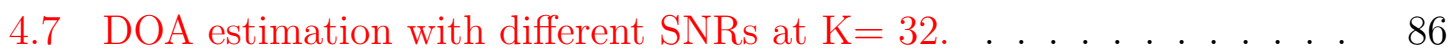

4.8 Spatial spectrum for RGDOA, DAS, MVDR and MUSIC for different closely spaced targets at 10 dB SNR and 16 snapshots. . . . . . . . . 87

4.9 Spatial spectrum for RGDOA, DAS, MVDR and MUSIC for different closely spaced targets at $-10 \mathrm{~dB}$ SNR and 8 snapshots. . . . . . . . .

4.10 Spatial spectrum of RGDOA for ULA and RSLA configurations for 16 snapshots under different SNRs. . . . . . . . . . . . . .

4.11 RMSE between true DOA and the RGDOA estimates for ULA and RSLA configurations for 8 snapshots for different SNRs. . . . . . . .

4.12 Spatial spectrum for RGDOA, DAS, MVDR and MUSIC for original data. . . . . . . . . . . . . . . . . . . 92

4.13 Spatial spectrum for RGDOA, DAS, MVDR and MUSIC for scaled SNR. 93

5.1 Block diagram of adaptive beamforming system. . . . . . . . . . . 97

5.2 Siegel distance between the asymptotic interference-plus-noise covariance and diagonally loaded, shrinkage, subspace, sparse and Riemannian geometry interference-plus-noise covariance matrices. . . . . . . 106

5.3 MVDR beamforming for different interference-plus-noise covariance matrix estimation techniques at $\mathrm{SNR}=10 \mathrm{~dB}$. . . . . . . . . . . . 107

5.4 MVDR beamforming for different interference-plus-noise covariance matrix estimation techniques at $\mathrm{SNR}=0 \mathrm{~dB}$. . . . . . . . . . . . 108 
5.5 MVDR beamforming for different interference-plus-noise covariance matrix estimation techniques at $\mathrm{SNR}=-10 \mathrm{~dB}$ with mismatch $\theta_{i}=28$ degrees. . . . . . . . . . . . . . . . . . . . . 109

5.6 MVDR beamforming for different interference-plus-noise covariance matrix estimation techniques for original data. . . . . . . . . . . 111

5.7 MVDR beamforming for different interference-plus-noise covariance matrix estimation techniques for scaled SNR. . . . . . . . . . . 112

A.1 Illustration of the classical beamforming structure. . . . . . . . . . . 131

A.2 Comparison of MVDR and MUSIC estimation methods. Two signals of equal power at an SNR of $20 \mathrm{~dB}$ arrive at an 8-element uniformly spaced array with an inter-element spacing equal to half a wavelength at angles 15 and 17 degrees respectively. . . . . . . . . . . 136 


\section{Nomenclature}

\begin{tabular}{|c|c|}
\hline Acronym & Meaning \\
\hline $\mathrm{ABHB}$ & angle-based hybrid Brauer \\
\hline $\mathrm{BD}$ & Brauer disc \\
\hline $\mathrm{CA}$ & cell-averaging \\
\hline CA-CFAR & cell-averaging constant false alarm rate \\
\hline CFAR & constant false alarm rate \\
\hline CPI & coherent processing interval \\
\hline CS & compressive sensing \\
\hline CUT & cell under test \\
\hline DARPA & Defense Advanced Research Projects Agency \\
\hline DAS & delay and sum \\
\hline DOA & direction of arrival \\
\hline DSSS & direct sequence spread spectrum \\
\hline EINC & estimated interference-plus-noise covariance \\
\hline $\mathrm{EO} / \mathrm{IR}$ & electro-optical/infrared \\
\hline ESPRIT & estimation of signal parameters via rotational invariance technique \\
\hline FFT & fast Fourier transform \\
\hline FHSS & frequency hoping spread spectrum \\
\hline FMCW & frequency-modulated continuous-wave \\
\hline
\end{tabular}




\begin{tabular}{|c|c|}
\hline GC & guard cells \\
\hline GLRT & generalized likelihood ratio test \\
\hline GPS & global positioning system \\
\hline $\mathrm{INC}$ & interference-plus-noise covariance \\
\hline HPD & Hermitian positive-definite \\
\hline KLB & Kullback-Leibler \\
\hline LRT & likelihood ratio test \\
\hline LW & Ledoit and Wolf \\
\hline MIMO & multiple input multiple output \\
\hline MSE & mean-squared error \\
\hline MVDR & minimum variance distortionless response \\
\hline MUSIC & multiple signal classification \\
\hline NP & Neyman-Pearson \\
\hline PA & phased array \\
\hline $\mathrm{PD}$ & positive definite \\
\hline $\mathrm{PDF}$ & probability density function \\
\hline PRD & passive radio detection \\
\hline PRI & pulse repetition interval \\
\hline $\mathrm{RBA}$ & regularized Burg algorithm \\
\hline $\mathrm{RBM}$ & Riemannian Brauer matrix \\
\hline $\mathrm{RC}$ & reference cells \\
\hline $\mathrm{RCS}$ & radar cross section \\
\hline $\mathrm{RD}$ & Riemannian distance \\
\hline $\mathrm{RG}$ & Riemannian geometry \\
\hline RMSE & root-mean-square error \\
\hline
\end{tabular}




\begin{tabular}{l|l} 
ROC & receiver operating characteristics \\
RS-INC & Riemannian space-based interference-plus-noise covariance \\
RSLA & randomly spaced linear array \\
SCR & signal-to-clutter ratio \\
SINR & signal-to-interference-plus-noise ratio \\
SNR & signal-to-noise ratio \\
SOI & signal of interest \\
SPLA & slightly perturbed linear array \\
THPD & Toeplitz Hermitian positive definite \\
UAS & unmanned aerial systems \\
UAV & unmanned aerial vehicles \\
ULA & uniform linear antenna \\
WSF & weighted spatial filter \\
\hline
\end{tabular}




\section{Chapter 1}

\section{Introduction}

\subsection{Drone Detection and Motivation}

Drones or unmanned aerial vehicles (UAV) are aircraft devices that are capable of flying above ground and carrying out missions without direct human piloting, not only for defense purposes but also for commercial, professional, and entertainment applications. Radio controlled drones can be obtained at an affordable price in a large variety of models, from a micro quad-copter, which can hold a small camera, up to a large multi-copter, which can carry a payload of several kilograms. Affordability by both common people and commercial organizations have led to an increase in use of drones in various applications. Drones can be used for surveillance missions, securing private facilities and borders, environmental inspection and as a flying hobby. On the other hand, drones can also be used as a spying tool, can be used to transport biological or chemical weapons to perform terrorist attacks or can be used to transport illegal drugs. Even friendly drones can be a potential risk if they get out of control or fly toward people, buildings or airports [1]. Therefore, a fast and reliable drone detection technique is need as a countermeasure for such threats.

In 2016, Defense Advanced Research Projects Agency (DARPA) released a public request for identifying new methods and approaches for fast detection, identification and neutralization of small unmanned aerial systems (UAS) [2]. A new protection program was initiated by DARPA to counter drone attacks by integrating the best solutions response received as responses to the public request. Fast and reliable drone detection technique would be a stringent requirement for such countermeasure systems. 
Several sensor-based passive detection techniques, such as acoustic [3], electrooptical/infrared (EO/IR) [4], passive radio [5] and other non sensor-based techniques like shooting and netting [6] are used to detect drones. Each of these techniques has it own capabilities in detecting drones. For instance acoustic systems can sense the oscillating parts of a potential target regardless of its size, EO/IR can detect the heat generated by a drone's motor and passive radio can monitor the communication channel between the drone and its operator. These techniques fail when the drones are auto-piloted and no radio-frequency waves are emanated or perform poorly in dark, misty or noisy environments.

Radar systems can provide long range sensing capabilities day and night in allweather and light conditions, along with the possibility of quantifying range, direction of arrival and velocity concurrently. These advantages has led to successful applications of radar in civil, automotive and military applications [7]. However, UAVs exhibit characteristics which are difficult to detect by typical conventional radar, such as low radar cross section (RCS), low-flying in urban areas, confusion with birds [8], unconventional flight patterns with small Doppler change and slow flying in a cluttered environment [9]. Also, modern radars might fail to detect drones in the presence of terrain masking effects, strong ground clutter or jamming by opponents. These issues cannot be solved with standard classical signal processing methods. One of the ways to overcome the signal processing challenges is to explore the drone detection problem from the Riemannian space perspective.

Riemannian space is a branch of differential geometry which is characterized by a mathematical object called a manifold. The Riemannian statistical manifold exploits the information geometry present in the space of probability distributions parametrized by their covariance matrices [10]. Signal processing in Riemannian space can be effective in the presence of small sample size due to its powerful statistical representations and entropy-based covariance matrix estimation. Riemannian metrics, such as distance and angle, are considered powerful tools in manipulating points on the Riemannian manifold. Each range bin of the received signal can be converted into a Toeplitz Hermitian positive definite matrix (THPD) residing on the the Riemannian manifold represents, where its eigenvalues can be localized using Brauer disc theorem [11]. The Toeplitz structure of the THPD covariance matrices are generated from uniform linear arrays as opposed to non-linear array configuration which generate non-Toeplitz covariance matrices. The drone detection and estimation 
problems can be improved by exploiting the statistical Riemannian manifold and the Brauer disc theorem.

\subsection{Problem Statement and Research Objective}

UAVs and micro-drones are becoming more available to the general public for leisure activities and exploited in commercial applications. However, there are growing concerns for accidental or even criminal misuses of these platforms. There are many challenges in detecting low flying drones in an urban contested environment [12]. Drones may not be detected by radar at all or may appear and disappear from radar's view. As a consequence, radar echo signals from drones might appear only in limited number of range bins (snapshots). This might lead to inaccurate estimation of the covariance matrix (rank-deficient) [13], and therefore leading to poor performance when classical signal processing methods for detection are considered. In addition, drones may vary in size from $10 \mathrm{~m}^{2}$ to $0.01 \mathrm{~m}^{2}$ with symmetrical shape and fluctuating RCS; hence, detection has to be performed under low signal-to-interference-plus-noise ratio (SINR) [8] conditions.

Drone detection using classical radar detection techniques and the standard estimation methods like subspace and sparsity may deteriorate under low SINR and limited number of received snapshots situations. The drone detection problem can be explored in the Riemannian space where every range bin is converted to a Hermitian positive definite covariance matrix representing a point on the Riemannian manifold $[14,15]$. Riemannian and Euclidean metrics can be exploited to improve the probability of detection, estimate the direction of arrival and the interference-plusnoise covariance matrix for beamforming applications.

\subsubsection{Hypothesis Statement}

It is hypothesised that the probability of drone detection and direction estimation can be further improved by employing Brauer disc theorem and exploiting signal processing in a Riemannian space. These approaches are believed to be effective in low SINR environment and for limited number of received radar snapshots.

The focus of this dissertation is to test the effectiveness of using Riemannian metrics in addition to Brauer disc theorem to improve the signal processing of drone 
detection, direction of arrival estimation, and interference-plus-noise covariance matrix for beamforming applications.

\subsection{Dissertation Organization}

As discussed in the previous sections, this dissertation focuses on drone detection and parameter estimation in a Riemannian space.

Chapter 2 focuses on literature review of drone detection techniques and introduces radar technology with a focus on multi-antenna radar systems and the concepts of CFAR detection. Also, the chapter provides a discussion on the Riemannian space of Hermitian positive definite (HPD) covariance matrices and Riemannian metrics, in addition to the eigenvalue localization techniques, such as, Gershgorin and Brauer disc theories.

Chapter 3 provides a background on fast Fourier transform, Riemannian distance and Kullback-Leibler CFAR detectors. In addition, it also proposes a new CFAR detector, Riemannian Brauer matrix (RBM), which is based on the Riemannian distance between Riemannian mean of the clutter-plus-noise THPD covariance matrices and potential targets' THPD covariance matrices. Furthermore, it proposes another new detector, angle-based hybrid Brauer (ABHB) CFAR, that exploits the Euclidean tangent space and Riemannian geodesical distances between Riemannian mean THPD covariance matrix, Riemannian median THPD covariance matrix and potential target THPD covariance matrix to compute the Riemannian angle at the potential target point on the Riemannian manifold. Furthermore, this chapter examines the performance of RBM-CFAR and ABHB-CFAR detectors for non-uniform linear array configuration in comparison with uniform linear array configuration. The performance of the RBM-CFAR and ABHB-CFAR detectors are validated using simulations and real data.

Chapter 4 presents a brief introduction to the direction of arrival (DOA) estimation techniques, multiple signal classification (MUSIC), estimation of signal parameters via rotational invariance technique (ESPRIT), minimum variance distortion-less response (MVDR) algorithms and compressive sensing with weighted spatial filter technique. In addition, it proposes a new Riemannian geometry-based direction of arrival (RGDOA) optimization technique, based on finding the minimum Riemannian distance between Riemannian mean of all THPD covariance matrices residing 
on the Riemannian manifold and steering vectors covariance matrices for DOA angles. Furthermore, this chapter investigates the affect of non-uniform linear array configuration on the RGDOA estimation technique in comparison with uniform linear array configuration. Simulations and real data analysis show that RGDOA maintained a robust performance for small sample size and low SNR.

Chapter 5 introduces a brief background on MVDR optimal beamforming and provides a brief introduction to the covariance matrix estimation techniques: sample covariance, adaptive diagonally loaded sample covariance, shrinkage algorithm, subspace-based interference-plus-noise covariance matrix estimation, sparsity-based interference-plus-noise covariance matrix estimation. In addition, it presents the Riemannian geometry based interference-plus-noise covariance matrix estimation technique. Also, it proposes a new interference-plus-noise covariance (INC) matrix estimation technique to increase the signal-to-noise ratio through MVDR beamforming. This technique is based on linear combination of THPD covariance matrices, where the weights are the max-min normalization of the Riemannian distance between the Riemannian mean and each of the THPD covariance matrices residing on the Riemannian manifold. Furthermore, simulations and real data analysis demonstrate the better performance of the INC in comparison with other covariance matrix estimation techniques through MVDR beamforming.

Chapter 6 concludes this dissertation and provides a brief summary of the contributions. Moreover, it provides future research direction.

\subsection{Contributions and Publications}

The following are the main contributions of the dissertation and the associated publications that have come out of this research work.

\subsubsection{Matrix CFAR Detector}

The contributions of Chapter 3 are:

- A new technique based on Brauer disc theorem to cluster clutter-plus-noise Hermitian positive definite covariance matrices.

- A Riemannian Brauer matrix (RBM) constant false alarm rate (CFAR) detector, which is based on the Riemannian distance between the Riemannian mean 
of the clutter-plus-noise cluster and any outliers (potential targets).

- An angle-based hybrid Brauer (ABHB) CFAR detector that utilizes Euclidean tangent space and Riemannian geodesical distances between Riemannian mean of the clutter-plus-noise cluster, Riemannian median of the clutter-plus-noise cluster and potential target points. The Riemannian detection angle at the potential target on the manifold is computed using law of cosines on the manifold.

The contributions of chapter 3 have been accepted for publication in IEEE Access journal:

- H. Chahrour, R. Dansereau, S. Rajan and B. Balaji, "Target detection through Riemannian Geometric Approach with Application to Drone Detection", accepted to IEEE Access.

\subsubsection{Direction of Arrival Estimation in Riemannian Space}

The contributions of Chapter 4 are:

- A Riemannian geometry direction of arrival (RGDOA) technique formulated as a distance optimization problem between the Riemannian mean and the steering vector covariance matrix for all possible direction of arrival angles.

- The solution for RGDOA distance optimization problem is a linear search for the minimum distance that corresponds to the direction of arrival estimate.

The following publication is related to this contribution:

- H. Chahrour, R. Dansereau, S. Rajan and B. Balaji, "Direction of Arrival Estimation using Riemannian Mean and Distance", 2019 IEEE Radar Conference (RadarConf), Boston, MA, USA, Apr. 2019, pp. 1-5.

\subsubsection{Covariance Matrix Estimation for Beamforming Appli- cations}

The contributions of Chapter 5 are:

- The estimated interference-plus-noise covariance (EINC) matrix formulated as a linear combination of the Toeplitz Hermitian positive definite (THPD) covariance matrices residing on the Riemannian manifold. 
- The weights of the linear combination operation based on the max-min normalization of the Riemannian distance between the Riemannian mean and each THPD covariance matrix on the manifold, where the largest Riemannian distance (potential target) will have a zero weight and the smallest distance will have maximum weight.

- The EINC improves the minimum variance distortionless response beamforming which leads to increase in the target's SNR.

This contribution was published in the following conference paper:

- H. Chahrour, R. Dansereau, S. Rajan and B. Balaji, "Improved Covariance Matrix Estimation using Riemannian Geometry for Beamforming Applications", 2020 International IEEE Radar Conference (RadarConf), Washington, DC, USA, Apr. 2020, pp. 693-697. 


\section{Chapter 2}

\section{Related Work and Fundamental Technology}

This chapter provides brief summaries of the state-of-art technologies used for drone detection. Also, it discusses briefly the concept of radar and its multi-antenna configurations, such as phased array and multiple input multiple output (MIMO) radar systems, and the concept of constant false alarm rate (CFAR) detection. Furthermore, this chapter defines UAV and its functionality and provides background information on Hermitian positive definite matrices and its Riemannian space. Finally, the chapter explores briefly the mathematical concept of eigenvalue localization techniques, such as Gershgorin and Brauer disc theorems.

\subsection{Drone Detection Techniques}

There are many techniques used to detect drones, such as acoustic, electro-opticalinfrared, passive radio and radar systems.

Acoustic sensors are based on the sound of acoustic wave energy produced by oscillating parts of a potential target, and the detection of target using acoustic sensors does not depend on the target size $[16,17]$. Acoustic systems usually consist of a microphone array that detects pressure changes created during wave transmission. Typically, drones produce hissing or buzz-like sound in frequencies ranging from 400 $\mathrm{Hz}$ to $8 \mathrm{kHz}$ and a unique acoustic signature can be extracted and separated from background noise using different audio analysis techniques [18]. The localization of drones using acoustic systems is based on estimating the time difference of arrival of the received audio signal [19]. Although acoustic sensors are easy to obtain, they 
fail to provide a precise location of a drone due to extreme environmental noise and limited detectable range.

Electro-optical-infrared (EO/IR) systems consist of high resolution digital video cameras, thermal and scanning sensors which generate a 2D heat image of potential targets [20]. EO/IR sensors collect environmental information in the visible and infrared light spectrum, which incorporates electromagnetic radiation with wavelengths between 400 nanometres and 1 millimetre. In addition, some EO/IR systems are capable of detecting, tracking and identifying drones by imaging the heat emitted by the drone's electric motor, paired with image analysis software [4]. Furthermore, EO/IR sensors are completely passive and are able to detect non-emitting (RF silent) drones. Even though EO/IR are efficient for detecting drones in both day and night, they cannot differentiate between complex background noise and targets and sometimes suffer from thermal image saturation which limits the efficiency of the detection process [21].

Passive radio detection (PRD) is based on a wideband radio spectrum analyser which monitors the two way communication channels between the drone and the operator [5]. PRD sensors analyze the radio signatures and modulations specific to drone signals and are capable of identifying certain drone models and manufacturers as well as locating the signal's transmission origin (operator) [22]. In addition, PRD sensors employ several signal processing techniques, like direction finding, received signal strength indicator and time difference of arrival to help localize the source of a drone associated signal. Although a PRD sensor can be very effective in detection drones, some radio control protocols used by drone communication links are hard to detect and break such as direct-sequence spread spectrum (DSSS) and frequencyhoping spread spectrum (FHSS) [23], and auto-piloted drones can be invisible to the PRD systems.

Radar systems can provide good capabilities in comparison with other detection systems. Radar systems can operate day and night, in all weather conditions, and can measure range, direction of arrival and Doppler shift. Conventional radar systems may encounter difficulties detecting low flying small complex targets, e.g. a small symmetrical shaped drone made of non-reflective material and with a small RCS [24]. Theoretically, modern radars can be an effective sensor in detecting a variety of targets in contested and cluttered environments. However, radar detection of small targets like drones present challenging factors, like low flying altitude with reduced Doppler shift and small RCS in urban areas [25]. In addition to challenging factors, drones 
need to be differentiated from biological targets, like birds and insects, which may have comparable RCS and flying pattern [8].

\subsection{Radar Technology}

Radar is a detection system that uses radio waves to detect objects and estimate their ranges, velocities and directions. Radar systems transmit electromagnetic waves toward an object and process echo waves to determine the range, velocity, direction of arrival and altitude [26]. It can be used to detect aircraft, spacecraft, drones, ships, ballistic or guided missiles, motor vehicles, clouds and hurricanes.

Radar systems generally consist of a transmitter which is responsible for producing an electromagnetic signal to be radiated into space by an antenna. Antennas are key components in radar systems which receive the reflected signal and feed it to the receiver. The receiver responsibility is to process the reflected signal to determine the geographical statistics of the object. The range is determined by calculating the time taken by the signal to travel from the radar to the target and back. The target's direction of arrival can be calculated from the angle that maximizes signal to noise ratio. In addition, the speed information of the target is extracted from the Doppler effect [27]. A block diagram of a typical conventional radar is depicted in Fig. 2.1.

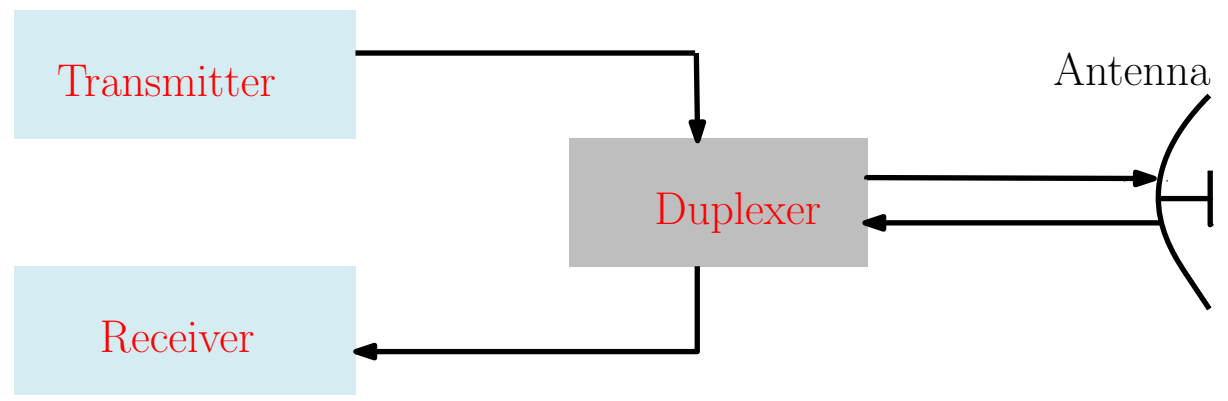

Figure 2.1: Block diagram of a typical conventional radar.

Radar systems can be categorized into two types: monostatic and bistatic. Monostatic radars have the transmitter and the receiver collocated while the transmitter and receiver of the bistatic radar are far apart [28]. In addition, radar systems can be further categorized into continuous waveform radar and pulse radar. The continuous waveform radar transmits a single continuous waveform while the pulse radar transmits multiple pulses every pulse repetition interval (PRI). Each PRI is evenly divided 
into a number of range cells or snapshots. The time delay $t_{d}$ of the reflected signal from a target is directly related to the distance between the radar and the target. The distance between the radar and the target is called the range $R_{T}$ and can be computed as [29]

$$
R_{T}=\frac{c t_{d}}{2}
$$

where $c$ is the speed of light and the factor 2 accounts for the round trip of the radar signal illuminating the target and back. The range resolution $\Delta r$ of the radar can be calculated using

$$
\Delta r=\frac{c}{2 B}
$$

where $B$ is the bandwidth of the radar. Figure 2.2 shows the range resolution and how the range is evenly divided into range cells for a typical radar. This dissertation focuses only on monostatic radar and the simulations are based on pulsed radar.

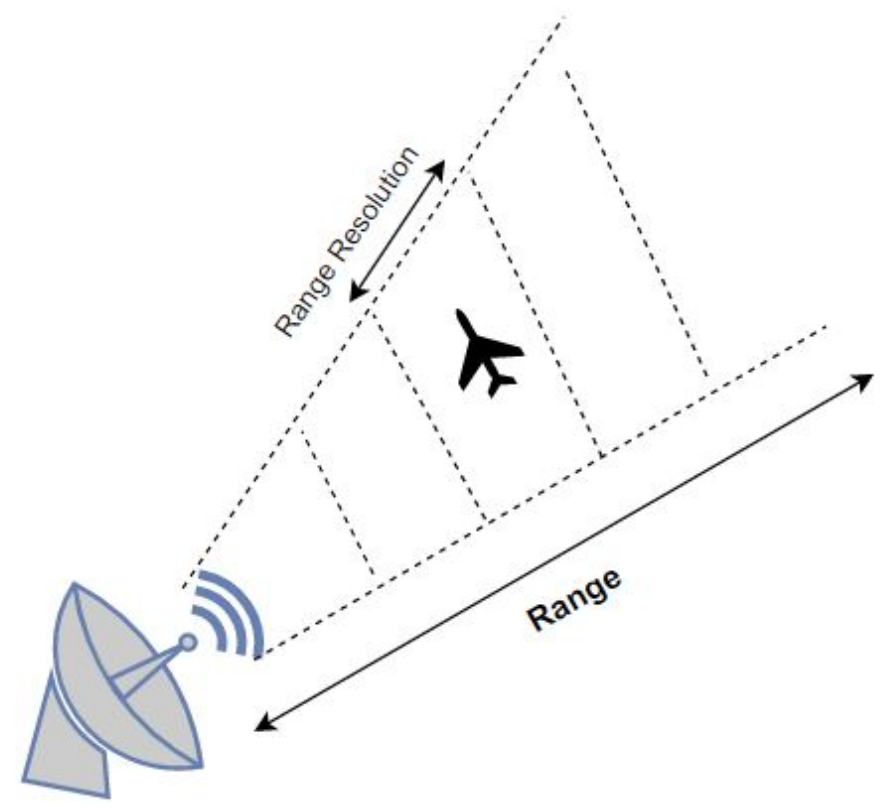

Figure 2.2: Radar range and range resolution.

In addition, the velocity of a moving target can be extracted from the Doppler frequency shift in the returned signal. The Doppler frequency shift is proportional to the component of velocity in the direction of the radar. The magnitude of the Doppler 
shift is maximum when the target is travelling toward or away from the radar. The Doppler shift is zero when the target is crossing orthogonally to the radar [30]. The Doppler frequency can be defined as

$$
f_{d}=\frac{2 v_{r}}{\lambda} \cos \zeta
$$

where $v_{r}$ is the radial speed of a target, $\lambda$ is the wavelength and $\zeta$ is the angle between velocity vector of the target and look angle of the radar. The Doppler frequency of a target can be extracted by applying a $p$-point discrete Fourier transform (DFT) for each discrete time index $n$ along the pulse repetition interval [31].

\subsubsection{Multi-Antenna Radar Systems}

Multi-antenna radar systems have been in service since World War II for military and aviation purposes. One of the most popular radar configurations is the phased-array (PA) radar system. PA radars utilize multiple transmitters and multiple receivers with collocated antenna elements. A single waveform is fed to multiple transmitter antenna elements that are equipped with a phase shifter each to create constructive and destructive interference to steer the transmitted energy toward a desired direction [32] as shown in Fig. 2.3. At the receiver array, the received signals can be beamformed by steering to the direction that maximizes the signal-to-noise ratio (SNR). There are two types of beamforming, analog and digital. Analog beamforming is based on phase shifters in the different receiver architectures and digital beamforming is based on adaptive processing. Digital beamforming offers several advantages over its analog counterpart, including the capability to steer multiple simultaneous beams [33] and the possibility to implement single and multiple sidelobe cancellers [34].

Another important type of multi-antenna radar system is the multiple-input multiple-output (MIMO) radar. MIMO radar also employs multiple transmitter and multiple receiver elements with multiple waveforms which can be correlated or uncorrelated (orthogonal) [35]. MIMO radars offer more degrees of freedom which lead to improved angular resolution, spatial diversity, larger area coverage, improved parameter identifiability, and more flexibility for transmit beampattern design [36] in comparison to the PA systems. Additionally, MIMO radars can synthesize larger virtual arrays which increase both the resolution and the number of targets that can be 


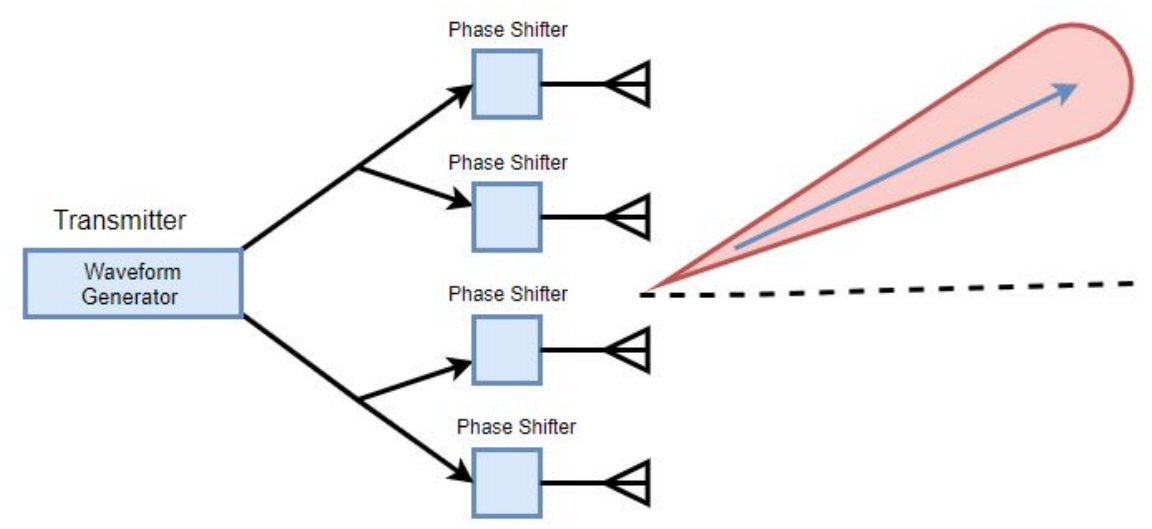

Figure 2.3: A typical 4 antenna element phased array radar transmitter.

detected [37]. On the other hand, MIMO radar illuminates a target with lower gain during transmission since it covers more volume space and it requires a longer coherent processing interval (CPI) to maintain the same SNR as a phased array radar [38]. In addition, MIMO radar increases the signal processing complexity due to extra matched filters at the receiver side [39]. A typical MIMO radar system is shown in Fig. 2.4.

Millimeter wave MIMO radars are ideal for surveillance tasks and allow high range resolution, however they are limited in detectable range due to their higher operating frequencies. Texas Instruments AWR1642 MIMO radar, which is based on frequency-modulated continuous-wave technology, can be used for many applications, such as, automotive and drone detection. On the other hand, pulsed radars provide range accuracy and better Doppler performance for low speed targets [26], like minidrones in comparison with continuous radar. This dissertation exploits the AWR1642 continuous returned signals to generate pulses with limited number of range bins to investigate the performance of detection and estimation techniques in Riemannian space. More details of the Texas Instruments AWR1642 MIMO radar are given in Sec. 3.7.

\subsubsection{Basic Radar Detection}

Radar can perform many functions such as detection, tracking and imaging. The primary function of radar is to detect targets or examine whether a radar measurement 


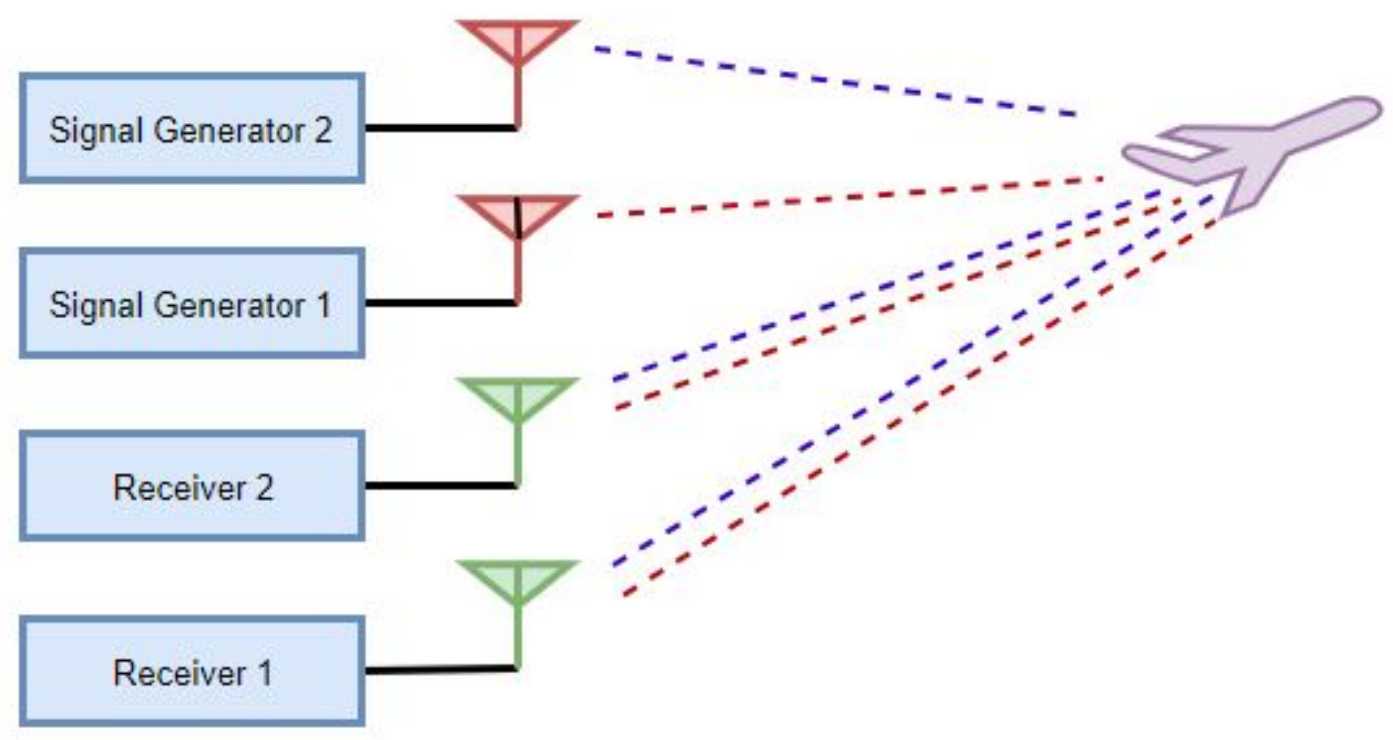

Figure 2.4: A typical $2 \times 2$ MIMO radar system.

is an echo from a target or simply represents background noise or clutter. Range detection is the simplest detection case where each range bin (fast time sample) for each pulse can be individually examined to determine if a target is present at the range corresponding to the range bin. The number of range bins can be in the thousands or more, so the radar can be making thousands of detection decisions per second [31]. The detection problem can be formulated as the hypotheses testing problem

$$
\left\{\begin{array}{l}
H_{0}: \mathbf{y}=\mathbf{n} \\
H_{1}: \mathbf{y}=\mathbf{s}+\mathbf{n} .
\end{array}\right.
$$

The first hypothesis $H_{0}$ represents the null hypothesis and $H_{1}$ represents the alternative hypothesis. Under the null hypothesis $H_{0}$, the received signal consists of background noise or clutter and under the alternative hypothesis $H_{1}$, the received signal contains target plus background noise or clutter. The detection analysis can be viewed from a statistical point of view where the probability density function (PDF) of the received signal can be examined under the $H_{0}$ and $H_{1}$ hypotheses [40]

- $p_{y}\left(y \mid H_{0}\right)$ PDF of $y$ when target not present; 
- $p_{y}\left(y \mid H_{1}\right)$ PDF of $y$ when target is present.

The classical approach of hypothesis testing is based on the Neyman-Pearson (NP) theorem and the Bayesian minimization of Bayes' risk. In context of radars, the NP criterion is designed to maximize the probability of detection $P_{d}$ under a constraint that the probability of false alarm does not exceed a set constant. This leads to the likelihood ratio test (LRT) decision rule [41]

$$
\frac{p_{y}\left(y \mid H_{1}\right)}{p_{y}\left(y \mid H_{0}\right)} \underset{H_{0}}{\stackrel{H_{1}}{\gtrless}}-T
$$

where $T$ is a Lagrange multiplier factor.

For the case of detection in Gaussian noise with variance $\beta^{2}$, the joint PDFs of $N$-dimensional normal distribution for both hypotheses can be written as

$$
\begin{gathered}
p_{y}\left(y \mid H_{0}\right)=\prod_{n=0}^{N-1} \frac{1}{\sqrt{2 \pi \beta^{2}}} \exp \left\{-\frac{1}{2}\left(\frac{y_{n}}{\beta}\right)\right\} \\
p_{y}\left(y \mid H_{1}\right)=\prod_{n=0}^{N-1} \frac{1}{\sqrt{2 \pi \beta^{2}}} \exp \left\{-\frac{1}{2}\left(\frac{y_{n}-m}{\beta}\right)\right\}
\end{gathered}
$$

where $m$ is the sample mean of the received signal. Applying the log-LRT, the probability of false alarm $P_{f}$ can be derived to be [42]

$$
P_{f}=\frac{1}{2}\left[1-\operatorname{erf}\left(\frac{T}{\sqrt{2 N \beta^{2}}}\right)\right]
$$

where $T$ is the detection threshold and erf is the error function. In addition, the $P_{d}$ can be derived to be

$$
P_{d}=\frac{1}{2} \operatorname{erfc}\left\{\operatorname{erfc}^{-1}\left(2 P_{f}\right)-\sqrt{\frac{\chi}{2}}\right\}
$$

where erfc is the complementary error function and $\chi$ is the estimated signal to noise ratio. Figure 2.5 illustrates a typical receiver operating characteristics (ROC) $P_{d}$ vs $P_{f}$ for radar detection for different SNR values in Gaussian noise for a non-fluctuating target. 


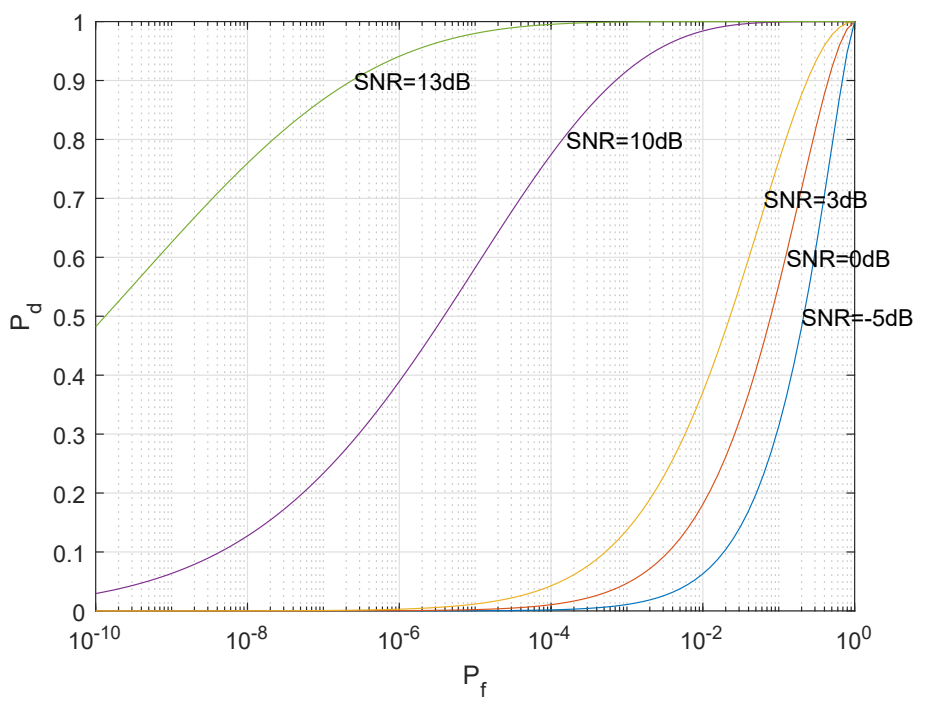

Figure 2.5: ROC curves non-fluctuating target.

\subsubsection{Constant False Alarm Rate Concept}

Noise and clutter power in real environments are non-stationary random processes varying with time. The concept of fixed threshold in detection problems may increase the probability of false alarms, while not achieving the desired probability of detection $P_{d}$. CFARs are designed to maintain the probability of false alarm $P_{f}$ of the background noise or clutter at a fixed level [41]. The CFAR detectors are designed to estimate the background power level from the samples around the sample under test to set a detection threshold adaptively that adjusts with the power level of the noise or clutter. The background noise in this dissertation is assumed to be independent Gaussian noise samples.

\section{CFAR Architecture}

The CFAR detector consists of four main elements: a cell under test (CUT), guard cells (GC), several reference cells (RC) and a CFAR multiplier $\alpha$, as shown in Fig. 2.6. These four elements assist the processor to vary threshold which follows the structure of background noise or clutter while maintaining a constant probability of false alarm [43]. The CUT is located in the middle of the CFAR window, where the threshold is going to be applied. The cells on either sides of CUT are considered as GCs. These GCs are not used to estimate the interference statistic and are used to eliminate any 


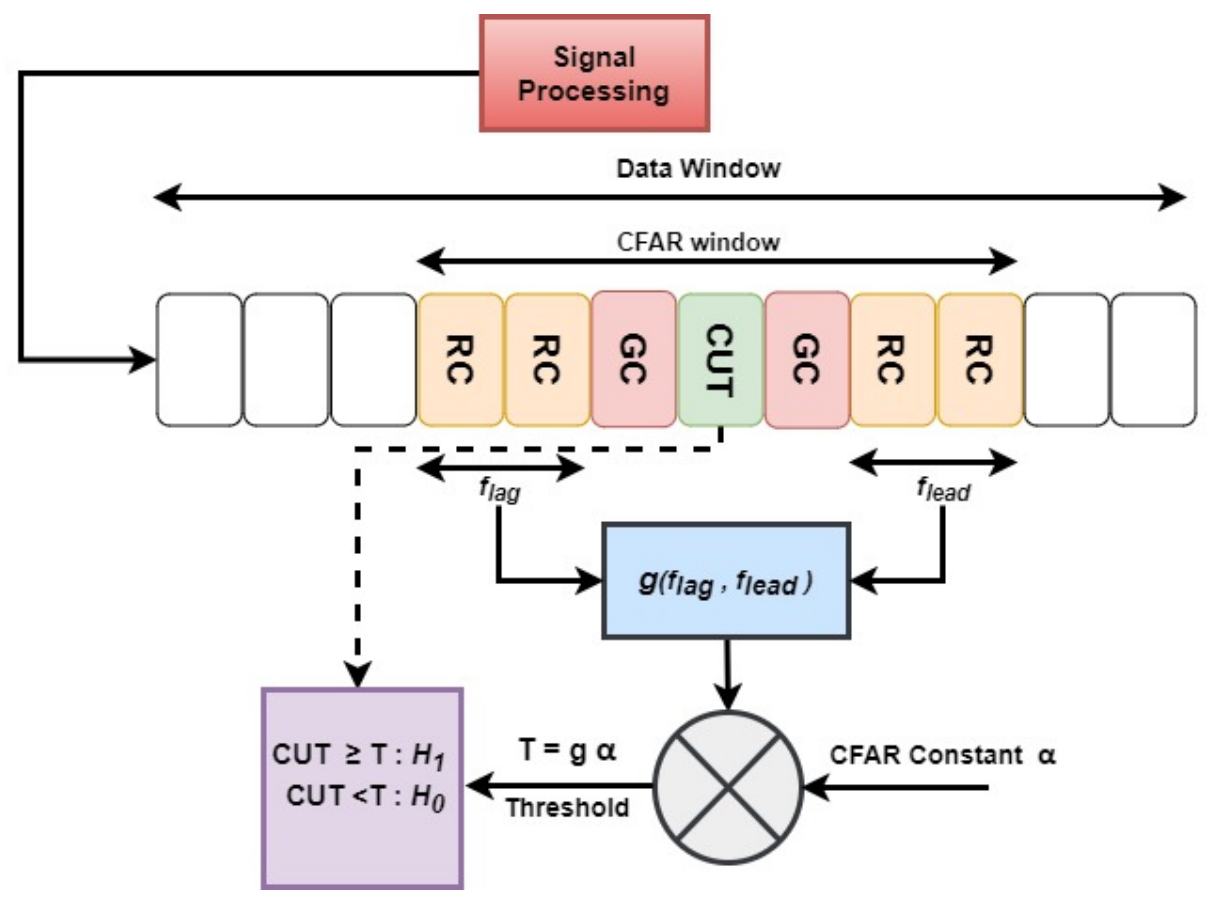

Figure 2.6: General CFAR processor.

spill over from the target if the target extends to more than one cell. This provides a better estimation of the background noise or clutter. The RCs are the outer cells of the CFAR processor. As shown in Fig. 2.6, $f_{\text {lag }}$ and $f_{\text {lead }}$ are RCs. $g\left(f_{\text {lag }}, f_{\text {lead }}\right)$ and are used to estimate the background noise or clutter. The more samples in $g\left(f_{\text {lag }}, f_{\text {lead }}\right)$, the better the estimation of the threshold. The CFAR multiplier $\alpha$ is also called CFAR constant and it is based on the desired probability of false alarm. Also, the threshold $T$ can be computed using the CFAR constant $\alpha$ and the background noise or clutter from the RCs. If the CUT exceeds the threshold, a successful detection will be declared.

\subsection{Unmanned Aerial Vehicle}

An unmanned aerial vehicle (UAV), well known as a drone, is defined as an aircraft without a human pilot on board that is replaced by a computer system and a radiolink. UAVs are a component of an unmanned aircraft system (UAS), which can be operated at various levels of autonomy, i.e. remote controlled, fully autonomous, and can carry payloads while on specific missions [44]. In addition to the payloads, drones can carry video cameras and different sensors such as radar, thermal and acoustic 
sensors to collect information and surveillance of areas of interest. In addition, they are equipped with a global positioning system (GPS) to determine the precise location that indicates the path of the mission [45].

Drones come in all sizes: some fit in the palm of a hand, while others are as large as full size aircraft. There are four main types of UAVs: fixed wing, which resembles airplanes, multi-rotor, single-rotor, which resembles a helicopter and fixed wing hybrid, which combine the benefits of fixed wing models and rotor based models.

UAVs can be exploited for defense, commercial, professional, and entertainment applications. Some of these functionality may include

- military surveillance missions,

- aerial photography for journalism and film,

- gathering information or supplying essentials for disaster management,

- search and rescue operations

- geographic mapping of inaccessible terrain and locations,

- building safety inspections,

- precision crop monitoring,

- unmanned cargo transport,

- law enforcement and border control surveillance, and

- storm tracking and forecasting hurricanes and tornadoes.

In this dissertation, a Yuneec Typhoon $\mathrm{H}$ drone is used to collect real data as shown in Fig. 2.7. 


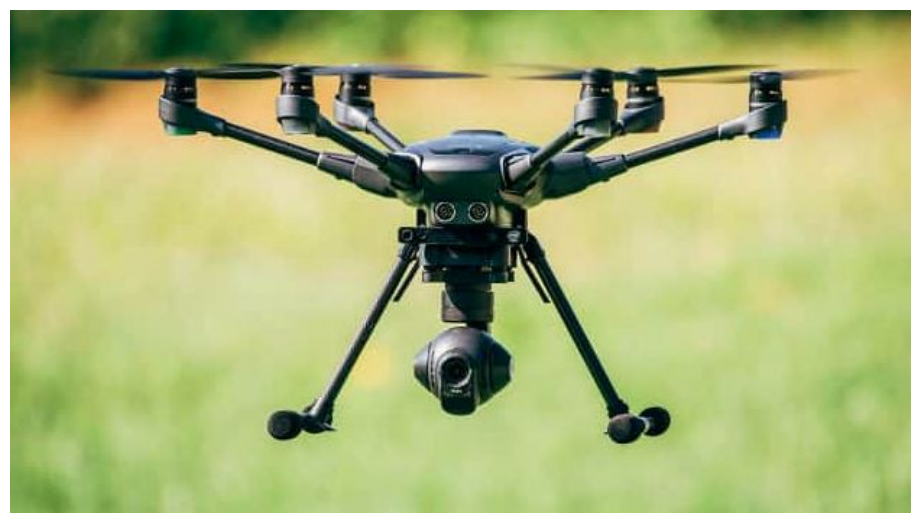

Figure 2.7: Yuneec Typhoon H drone.

\subsection{Regularized Burg Algorithm}

Burg's method for maximum entropy spectral analysis plays a key role in spectral estimation, speech processing and radar covariance matrix estimation [46, 47]. Burg algorithm estimates the autoregressive model parameters without computing a correlation matrix and solving Yule-Walker equation while satisfying Levinson recursion. It provides higher spectral resolution than conventional spectral estimation methods for small data records [48]. The reflection coefficients are based on autoregressive model order and minimizing the sum of mean squared values of the forward and backward prediction errors. The regularized Burg algorithm (RBA) estimates the reflection coefficients through regularized method based on Bayesian adaptive spectral estimation. This algorithm maintains the lattice structure which provides robustness against coefficient value perturbation and less round off noise [49]. The RBA will be exploited to estimate the reflection coefficients for every snapshot of the radar observation data. From the reflection coefficients, we can estimate the Toeplitz Hermitian positive definite (THPD) covariance matrix for each radar snapshot.

Consider an $N$ element received baseband complex radar snapshot which can be written as $\mathbf{x}=\left(x_{0}, \ldots, x_{N-1}\right)$ and is realized as a multivariate stationary Gaussian process with zero mean, $\mathbf{x} \sim \mathcal{C N}(0, \mathbf{R})$, which can be written as

$$
P(\mathbf{x} \mid \mathbf{R})=\frac{1}{\sqrt{\pi^{N} \operatorname{det}(\mathbf{R})}} \exp \left(-\mathbf{x}^{H} \mathbf{R}^{-1} \mathbf{x}\right)
$$

where $P(\mathbf{x} \mid \mathbf{R})$ is the probability density function (PDF) of a circular Gaussian random vector and $\mathbf{R}$ is a THPD covariance matrix which can be estimated using Algorithm 
$1[14,46,50]$. In Algorithm 1, $\psi_{1}$ is a regularization parameter and $N$ represents the number of reflection coefficients and autoregressive model order.

Algorithm 1 Regularized Burg Algorithm [14,46,50]

Initialization

$$
\begin{aligned}
f_{0}(k) & =z(k), k=1, \ldots, N, \\
b_{0}(k) & =z(k), \\
P_{0} & =\frac{1}{N} \sum_{k=1}^{N}|z(k)|^{2} \text { and } a_{0}^{(0)}=1
\end{aligned}
$$

for $n=1$ to $\mathrm{N}-1$ do

$$
\mu_{n}=-\frac{\frac{2}{N-n} \sum_{k=n+1}^{N} f_{n-1}(k) \bar{b}_{n-1}(k-1)+\delta_{n}}{\frac{1}{N-n} \sum_{k=n+1}^{N}\left\{\left|f_{n-1}(k)\right|^{2}+\left|b_{n-1}(k-1)\right|^{2}\right\}+\delta_{d}},
$$

with

$$
\begin{aligned}
\beta_{k}^{(n)} & =\psi_{1}(2 \pi)^{2}(k-n)^{2}, \\
\delta_{n} & =2 \sum_{k=1}^{n-1} \beta_{k}^{(n)} a_{k}^{(n-1)} a_{n-k}^{(n-1)}, \\
\delta_{d} & =2 \sum_{k=0}^{n-1} \beta_{k}^{(n)}\left|a_{k}^{(n-1)}\right|^{2}
\end{aligned}
$$

and

$$
\begin{gathered}
\left\{\begin{array}{l}
a_{0}^{(n)}=1, \\
a_{k}^{(n)}=a_{k}^{(n-1)}+\mu_{n} \bar{a}_{n-k}^{(n-1)}, k=1, \ldots, n-1 \\
a_{n}^{(n)}=\mu_{n}
\end{array}\right. \\
\begin{cases}f_{n}(k)=f_{n-1}(k)+\mu_{n} b_{n-1}(k-1), \\
b_{n}(k)=b_{n-1}(k-1)+\mu_{n}^{*} f_{n-1}(k) .\end{cases}
\end{gathered}
$$

end for

The reflection coefficients $\mu_{k}$ can be used to calculate $r_{k}$, which represent the elements of the THPD covariance matrix $\mathbf{R}$ of snapshot $\mathbf{x}$ written as 


$$
\mathbf{R}=\left[\begin{array}{cccc}
r_{0} & \bar{r}_{1} & \cdots & \bar{r}_{N-1} \\
r_{1} & r_{0} & \cdots & \bar{r}_{N-2} \\
\vdots & \ddots & \ddots & \vdots \\
r_{N-1} & \cdots & r_{1} & r_{0}
\end{array}\right]
$$

The $r_{k}$ are computed using the following equations [51]

$$
\begin{aligned}
& r_{0}=P_{0}, r_{1}=-P_{0} \mu_{1}, \\
& r_{k}=-P_{k-1} \mu_{k} \boldsymbol{\alpha}_{k-1}^{T} \mathbf{J}_{k-1} \mathbf{R}_{k-1}^{-1} \boldsymbol{\alpha}_{k-1}, 2 \leqslant k \leqslant N-1
\end{aligned}
$$

where

$$
\begin{aligned}
\boldsymbol{\alpha}_{k-1}= & {\left[\begin{array}{c}
r_{1} \\
\vdots \\
r_{k-1},
\end{array}\right], \mathbf{J}_{0}=1, \mathbf{J}_{1}=\left[\begin{array}{ll}
0 & 1 \\
1 & 0
\end{array}\right], } \\
\mathbf{J}_{k-1}= & {\left[\begin{array}{cccc}
0 & 0 & \cdots & 1 \\
0 & \cdots & 1 & 0 \\
& \cdots & \cdots & \\
1 & \cdots & 0 & 0
\end{array}\right], } \\
\mathbf{R}_{k-1}= & \mathbf{R}(2: k, 1: k-1), P_{k-1}=P_{0} \prod_{i=1}^{k-1}\left(1-\left|\mu_{i}\right|^{2}\right) .
\end{aligned}
$$

The RBA guarantees the Toeplitz structure for every THPD covariance matrix regardless of the antenna array configuration. Uniform linear array produce Toeplitz covariance matrices, while non-linear array configurations produce non-Toeplitz covariance matrices. In this dissertation, every radar range bin will be converted to a THPD covariance matrix. 


\subsection{Hermitian Positive Definite Matrices}

Positive definite (PD) matrices can be applied in many applications, such as complex analysis, vibrations of mechanical systems, applied matrix theory and radar signal processing [52]. Covariance and Hessian matrices are good examples of positive definite matrices. In addition, PD matrices used in optimization algorithms and linear regression models [53].

Given a matrix $\mathbf{A} \in \mathbb{C}^{n \times n}$, the scalars $\lambda_{i} \in \mathbb{C}, i=1, \ldots, n$, are called the eigenvalues of matrix $\mathbf{A}$. Let us also consider the singular matrix, $\mathbf{A}-\lambda_{i} I_{n}$, where $I_{n}$ is the identity matrix of size $n$. Any vector $\mathbf{v}_{i} \in \mathbb{C}$, which satisfies $\mathbf{A} \mathbf{v}_{i}=\lambda_{i} \mathbf{v}_{i}$, is called an eigenvector of $\mathbf{A}$ corresponding to the eigenvalue $\lambda_{i}$. PD matrices have the following properties [54]:

- $\mathbf{a}^{T} \mathbf{A} \mathbf{a}>0$ for all $\mathbf{a} \in \mathbb{C}^{n}$.

- $\mathbf{A}=\mathbf{L L}^{T}$ is called Cholesky factorization, $\mathbf{L} \in \mathbb{C}^{n \times n}$ is a lower triangular matrix.

- if $\mathbf{A}$ is an $n \times n$ PD matrix and $\mathbf{B}$ is an $n \times n$ PD matrix, then $\mathbf{A}+\mathbf{B}$ is an PD matrix.

- All eigenvalues of PD matrices are positive real numbers

- The determinant and trace of PD matrices are positive real number.

- Principal submatrix of a PD matrix is PD.

- The inverse of a PD matrix is also PD.

- The largest element of PD matrix is on the main diagonal.

A positive definite matrix is Hermitian for which all eigenvalues are real positive. In addition, the RBA from the previous section allows the Hermitian positive definite (HPD) matrices to be Toeplitz matrices as shown in (2.11). In this dissertation, we will only consider the complex definition of Toeplitz Hermitian positive definite (THPD) matrix for every received snapshot. 


\subsection{Differential Geometry and Manifolds}

Differential geometry is a branch of mathematics that exploits the techniques of differential calculus, integral calculus, linear algebra and multi-linear algebra to solve problems in higher spaces [55]. Differential geometry is connected with differential topology and geometric differential equations. There are many diverse applications of differential geometry in many areas, such as biology, mathematical finance, machine learning and statistical probability. Einstein applied differential geometry generalization and Finsler geometry to formulate the theory of general relativity.

Since the late $19^{\text {th }}$ century, differential geometry has generally focused on geometric structures called differential manifolds. The most popular geometrical structures used are Grassmannian, Kähler and Riemannian manifolds. Grassmannian manifold is the space of lines through the origin generated by parameterizing dimensional linear subspaces of the higher dimensional vector space [56]. Kähler manifold [57] is a manifold with three mutually compatible structures: a complex structure, a Riemannian structure and a symplectic structure. Riemannian manifold is equipped with a positive definite inner product on every point of the tangent space [58]. In addition, Riemannian submanifold inherits the Riemannian properties of a Riemannian manifold especially the Riemannian metric. In contrast to Grassmannian and Kähler manifolds, Riemannian metric, such as, distance, which is the information geometry distance between Hermitian positive definite matrices on the Riemannian manifold can be an effective tool in signal processing. This dissertation focuses only on the Riemannian manifold and its metrics.

\subsubsection{Riemannian Manifold}

Riemannian geometry is a branch of differential geometry, which is characterized by a mathematical object called a manifold. A Riemannian manifold consists of a topological space with some similarity to a Euclidean space. Every point on the Riemannian manifold has a neighbourhood for which there exists a homeomorphism ${ }^{1}$. In addition, a Riemannian manifold is a differentiable manifold in which each tangent space has an inner product $g$ that varies smoothly from point to point $[58,59]$. A Riemannian metric on $\mathcal{M}$ allows one to measure lengths of smooth paths in $\mathcal{M}$ by

\footnotetext{
${ }^{1}$ Homeomorphism is a continuous function between topological spaces that has a continuous inverse function and the mappings that preserve all the topological properties of a given space.
} 
taking the infimum of the lengths of smooth paths between two points.

\section{Tangent Spaces on Riemannian manifold}

Tangent spaces can be defined at every point on the Riemannian manifold. That is, for each $\mathbf{P} \in \mathcal{M}$, we have a tangent space $\mathbf{T}_{p} \mathcal{M}$. Figure 2.8 shows the tangent space of $\mathcal{M}$ at $\mathbf{P}$.

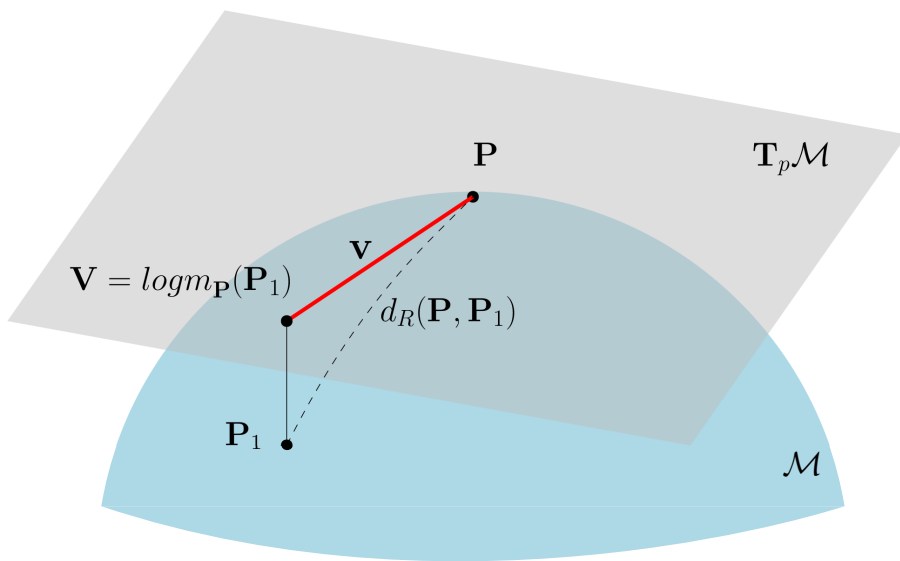

Figure 2.8: Tangent space of the manifold $\mathcal{M}$ at point $\mathbf{P}$ and the projection of $\mathbf{P}_{1}$ onto the tangent of point $\mathbf{P}$.

The concept of tangent space can be used to manipulate HPD covariance matrices in Euclidean space by using a Riemannian matrix log map operator. Any HPD covariance matrix on the manifold can be projected onto a tangent plane of a different HPD covariance matrix, as shown in Fig. 2.8. The projection can be achieved using [60]

$$
\begin{aligned}
\mathbf{V} & =\operatorname{Logm}_{\mathbf{P}}\left(\mathbf{P}_{1}\right) \\
& =\mathbf{P}^{1 / 2} \operatorname{logm}\left(\mathbf{P}^{-1 / 2} \mathbf{P}_{1} \mathbf{P}^{-1 / 2}\right) \mathbf{P}_{1 / 2}
\end{aligned}
$$

where $\operatorname{logm}(\cdot)$ is the matrix logarithmic map and $\mathbf{P}$ and $\mathbf{P}_{1}$ are HPD covariance matrices on the Riemannian manifold. Furthermore, the projection back to the space of HPD covariance matrices can be done using the affine-invariant metric [61] and 
matrix exponential operator

$$
\begin{aligned}
\mathbf{P}_{1} & =\operatorname{Expm}_{\mathbf{P}}(\mathbf{V}) \\
& =\mathbf{P}^{1 / 2} \operatorname{expm}\left(\mathbf{P}^{-1 / 2} \mathbf{V} \mathbf{P}^{-1 / 2}\right) \mathbf{P}^{1 / 2}
\end{aligned}
$$

where $\operatorname{expm}(\cdot)$ is the matrix exponential map. Both operators are crucial in the manipulation of HPD covariance matrices residing on the Riemannian manifold.

\section{Riemannian Metric}

A Riemannian manifold $(\mathcal{M}, g)$ is a differentiable manifold equipped with a Riemannian metric $g$. The metric $g$ is defined by a local inner product on tangent vectors [62]

$$
g_{\mathbf{P}}(\cdot, \cdot): \mathbf{T}_{\mathbf{p}} \mathcal{M} \times \mathbf{T}_{\mathbf{p}} \mathcal{M} \rightarrow \mathbb{R}, \quad \mathbf{P} \in \mathcal{M}
$$

such that, for all differentiable vector fields $\mathbf{X}$ and $\mathbf{Y}$, the application

$$
\mathcal{M} \rightarrow \mathbb{R}, \quad \mathbf{P} \mapsto g(\mathbf{X}(\mathbf{P}), \mathbf{Y}(\mathbf{P}))
$$

is differentiable. The Riemannian metric enables us to define lengths of curves on the Riemannian manifold between two HPD covariance matrices. The Riemannian distance $d_{R}$ can be defined as [10]

$$
d_{\mathrm{R}}^{2}\left(\mathbf{P}, \mathbf{P}_{1}\right)=\left\|\operatorname{logm}\left(\mathbf{P}^{-1 / 2} \mathbf{P}_{1} \mathbf{P}^{-1 / 2}\right)\right\|_{F}^{2}=\sum_{k=1}^{l} \log ^{2}\left(\lambda_{k}\right)
$$

where $\|\cdot\|_{F}$ is the Frobenius norm, $\left\{\lambda_{1}, \ldots, \lambda_{l}\right\}$ are the eigenvalues of $\mathbf{P}^{-1 / 2} \mathbf{P}_{1} \mathbf{P}^{-1 / 2}$ and $l$ is the number of eigenvalues. The Riemannian distance represents the geodesical distance taking in consideration the curvature of the Riemannian manifold as shown in Fig. 2.9.

\section{Siegel Distance}

The Siegel distance can be defined as the geodesical distance between two distributions parametrized by their covariance matrices [63]. Siegel distance can be measured on Riemannian manifold where each covariance matrix is a HPD. The Siegel distance 


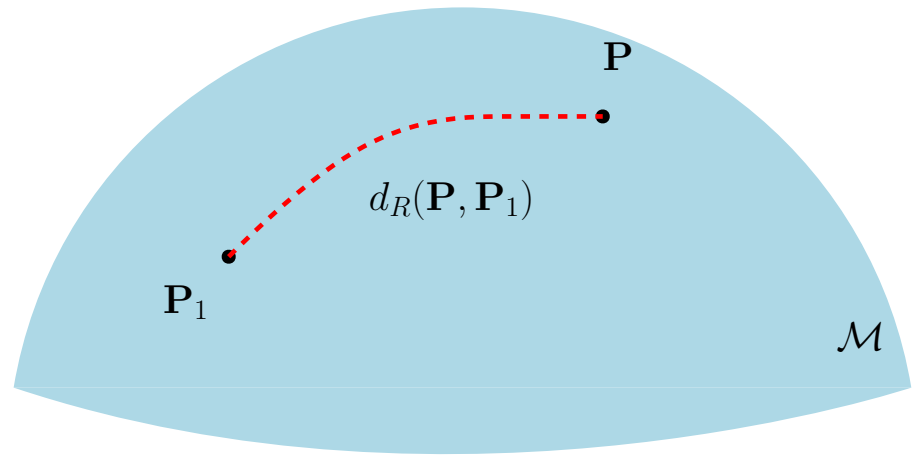

Figure 2.9: Riemannian distance between two HPD covariance matrices $\mathbf{P}$ and $\mathbf{P}_{1}$ on the Riemannian manifold $\mathcal{M}$.

can be defined by [64]

$$
d_{\mathrm{S}}^{2}\left(\mathbf{P}, \mathbf{P}_{1}\right)=\left\|\operatorname{logm}\left(\mathbf{P}^{-1 / 2} \mathbf{P}_{1} \mathbf{P}^{-1 / 2}\right)\right\|_{F}^{2}=\sum_{k=1}^{l} \log ^{2}\left(\lambda_{k}\right)
$$

The Siegel distance, also referred to as the Riemannian distance defined in (2.18), represents the geodesical distance between two HPD covariance matrices on the space of Hermitian positive definite matrices [63]. Also, they measure the distance between multivariate normal distributions parametrized by their covariance matrices on higher dimensional manifold.

\section{Riemannian Mean and Median of HPD Covariance Matrices}

Riemannian mean and median can be effective mathematical and computational tools for analyzing high dimensional data [65]. Unlike the arithmetic mean and median, which are usually used in statistical signal processing, the Riemannian (geometric) mean and median are based on the on the minimum sum squared of the Riemannian distances to all HPD covariance matrices on the manifold.

Each point on the Riemannian manifold is a HPD covariance matrix $\mathbf{R}$ which belongs to a convex symmetric cone set residing on the manifold

$$
\mathbb{P}=\{\mathbf{R} \in \mathcal{M}, \mathbf{R} \succ 0\}
$$

where $\mathcal{M}=\left\{\mathbf{R}, \mathbf{R}^{H}=\mathbf{R}\right\}$ defines a Riemannian space, $\succ$ denotes Hermitian positive definite matrix and the quadratic form of $\mathbf{R}, a^{H} \mathbf{R} a>0, \forall a \in \mathbb{C}$. The process of 
acquiring a Riemannian mean (geometric) of $K$ HPD matrices is an iterative gradient algorithm based on the Jacobi field and exponential map, also known as Karcher Barycenter algorithm [50], which can be expressed as

$$
\mathbf{A}_{t+1}=\mathbf{A}_{t}^{1 / 2} \exp \left\{\epsilon \sum_{k=1}^{K} \operatorname{logm}\left(\mathbf{A}_{t}^{-1 / 2} \mathbf{R}_{k} \mathbf{A}_{t}^{-1 / 2}\right)\right\} \mathbf{A}_{t}^{1 / 2}
$$

where $t$ is the iteration index, $\epsilon$ is the step size, $K$ is the number of snapshots, $\left\{\mathbf{R}_{1}, \ldots, \mathbf{R}_{K}\right\}$ is a set of HPD matrices, $\mathbf{A}_{1}$ is the arithmetic mean of the HPD matrices and the converged $\mathbf{A}_{t+1}$ is the Riemannian mean $\mathbf{R}_{r m}$. Figure 2.10 shows the HPD covariance matrices and their Riemannian mean on Riemannian manifold. Similarly, the Riemannian median can be defined using the same parameters as the Riemannian mean [66]

$$
\mathbf{A}_{t+1}=\mathbf{A}_{t}^{1 / 2} \exp \left\{\epsilon \sum_{k=1}^{K} \frac{\operatorname{logm}\left(\mathbf{A}_{t}^{-1 / 2} \mathbf{R}_{k} \mathbf{A}_{t}^{-1 / 2}\right)}{C}\right\} \mathbf{A}_{t}^{1 / 2}
$$

where $C=\left\|\operatorname{logm}\left(\mathbf{A}_{t}^{-1 / 2} \mathbf{R}_{k} \mathbf{A}_{t}^{-1 / 2}\right)\right\|_{F},\|\cdot\|_{F}$ is the Frobenius norm and the converged $\mathbf{A}_{t+1}$ is the Riemannian median $\mathbf{R}_{r m e d}$.

The Riemannian mean and median are the corner stones for a new radar detection technique based on the Brauer disc theorem and the law of cosines on the Riemannian manifold proposed in this dissertation. 


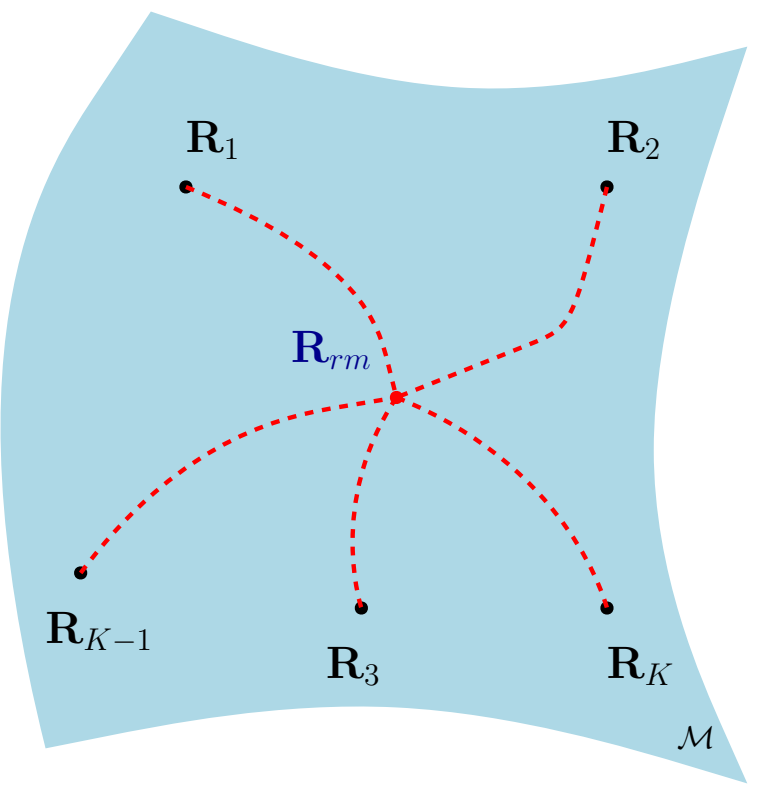

Figure 2.10: Riemannian mean of the HPD covariance matrices on the Riemannian manifold $\mathcal{M}$.

\subsection{Eigenvalue Localization Techniques}

The Gershgorin disc theorem [67] is a well-known and an efficient method for determining the inclusion domain of the eigenvalues of a matrix in terms of its entries. This means that all eigenvalues of a matrix must lie in a union of discs, each centred at a diagonal element of the matrix and having a radius equal to the corresponding deleted row sum [68]. Specifically, given an $n \times n$ matrix $A$ whose entries are denoted by $a_{i j}$, we define the Gershgorin disc as

$$
D_{i}=\left\{z \in \mathbb{C}:\left|z-a_{i i}\right| \leqslant \sum_{j \neq i}\left|a_{i j}\right|\right\}
$$

Figure 2.11 shows typical Gershgorin discs for $3 \times 3$ matrix with real eigenvalues. The Gershgorin disc theorem can also be applied to THPD covariance matrices. The Toeplitz property makes all the discs of the THPD matrix share the same disc centre. In addition, the Hermitian property imposes disc overlapping. The Gershgorin disc theorem for Toeplitz Hermitian $\mathbf{R}$ can be defined using the following theorem.

Theorem 2.7.1 [11][The Gershgorin disc theorem] 


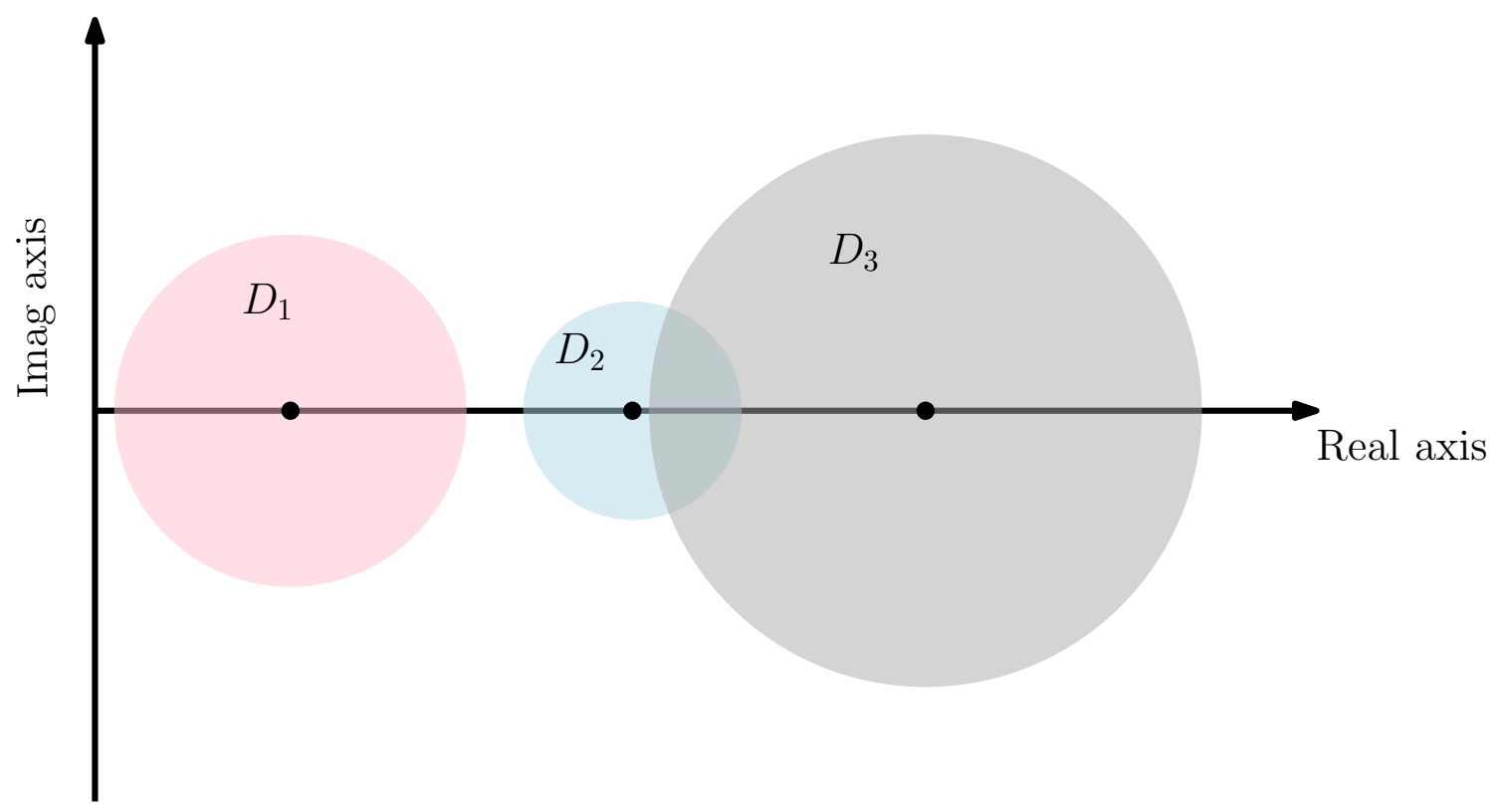

Figure 2.11: Typical Gershgorin discs for real eigenvalues.

Let $\mathbf{R}=\left[r_{i j}\right] \in \mathbb{C}^{n \times n}$ be a Toeplitz matrix, $r_{i i}=\bar{r}, n \geqslant 2$ and $\sigma(\mathbf{R})$ be spectrum of R. Then,

$$
\begin{aligned}
\sigma(\mathbf{R}) \subseteq \Gamma(\mathbf{R}) & =\bigcup_{i=1}^{\left\lceil\frac{n}{2}\right\rceil} \Gamma_{i}(\mathbf{R}) \\
\text { where } \Gamma_{i}(\mathbf{R}) & =\left\{z \in C:|z-\bar{r}| \leq \max _{i \in N} a_{i}(\mathbf{R})\right\} \\
a_{i}(\mathbf{R}) & =\sum_{i \neq j}\left|r_{i j}\right| \text { and } N=1,2, \ldots, n \\
\left\lceil\frac{n}{2}\right\rceil & =\left\{\begin{array}{ccc}
\frac{n}{2}, & \text { if } n \text { is even } \\
\frac{n+1}{2}, & \text { if } n \text { is odd }
\end{array}\right.
\end{aligned}
$$

$\Gamma(\mathbf{R})$ is the Gershgorin disc set of the covariance matrix $\mathbf{R}$. Although the Gershgorin disc theorem provides a good inclusion bound for the eigenvalues, the Brauer disc theory provides a tighter inclusion bound, which depends on the row and column summations for the region of eigenvalues.

Theorem 2.7.2 [11][The Brauer disc theorem]

Let $\mathbf{R}=\left[r_{i j}\right] \in \mathbb{C}^{n \times n}$ be a Toeplitz matrix, $r_{i i}=\bar{r}, n \geqslant 2$ and $\sigma(\mathbf{R})$ be spectrum of 
R. Then,

$$
\begin{aligned}
\sigma(\mathbf{R}) \subseteq \Upsilon(\mathbf{R}) & =\bigcup_{i=1}^{\left\lceil\frac{n}{2}\right\rceil} \Upsilon_{i}(\mathbf{R}) \\
\text { where } \Upsilon_{i}(\mathbf{R}) & =\left\{z \in C:|z-\bar{r}| \leq \max _{i, j \in N, i \neq j} \sqrt{a_{i}(\mathbf{R}) a_{j}(\mathbf{R})}\right\} \\
a_{i}(\mathbf{R}) & =\sum_{i \neq j}\left|r_{i j}\right| \text { and } N=1,2, \ldots, n \\
\left\lceil\frac{n}{2}\right\rceil & =\left\{\begin{array}{lll}
\frac{n}{2}, & \text { if } n \text { is even } \\
\frac{n+1}{2}, & \text { if } n \text { is odd }
\end{array}\right.
\end{aligned}
$$

$\Upsilon(\mathbf{R})$ is the Brauer disc set of the covariance matrix $\mathbf{R}$. Based on [69], the Brauer eigenvalue inclusion set is tighter than the Gershgorin set with $\Upsilon(\mathbf{R}) \subseteq \Gamma(\mathbf{R})$ as shown in Fig 2.12. In addition, the maximum eigenvalue of $\mathbf{R}$ is bounded by the largest Gershgorin and Brauer discs [70]. The Brauer disc theorem will be used to establish a cluster bound around clutter plus noise THPD covariance matrices and the potential targets' THPD covariance matrices.

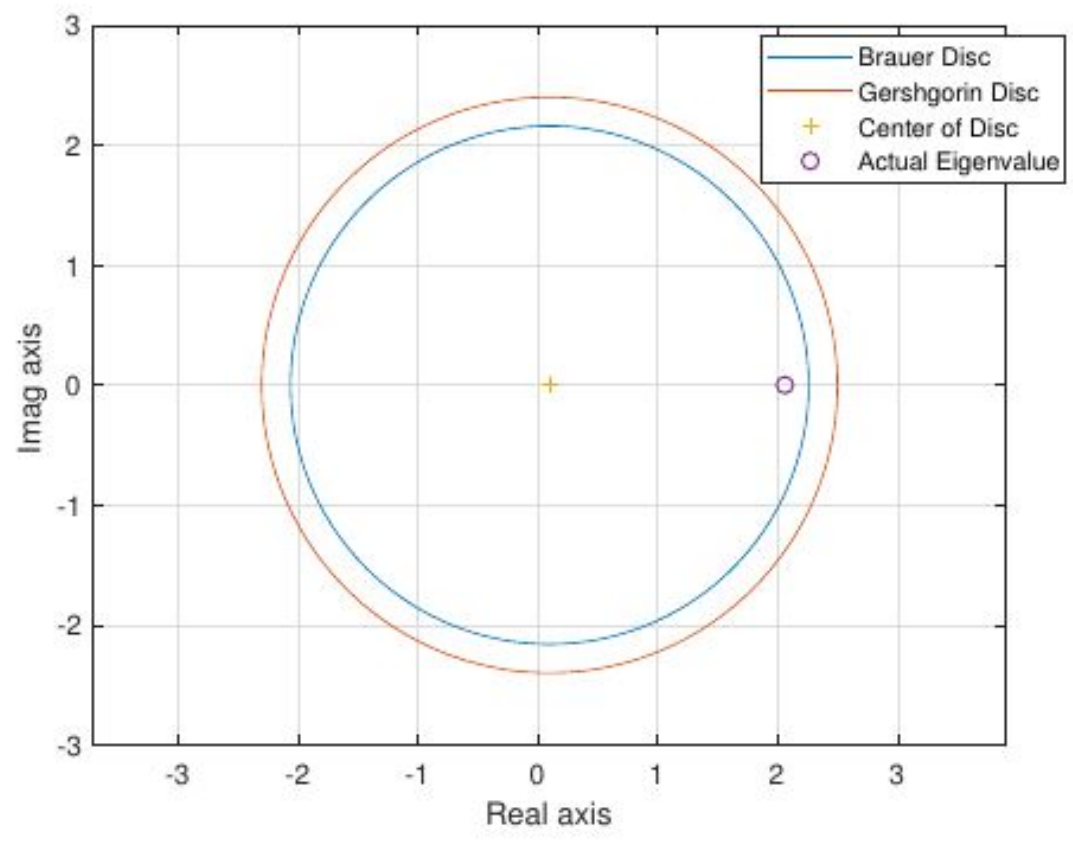

Figure 2.12: Gershgorin and Brauer discs for the maximum eigenvalue of covariance matrix $\mathbf{R}$. 


\subsection{Conclusion}

In this chapter, we have discussed the pros and cons of drone detection technologies. The radar theory with multi-antenna configurations and the concept of CFAR

detection have been introduced. In addition, the concept of Riemannian space of HPD covariance matrices a focus on the Riemannian mean, the Riemannian median and the Riemannian distance has been put forward. Moreover, the mathematical concepts of the Gershgorin and the Brauer disc theorems have been presented. The mathematical concepts discussed in this chapter will be used in this dissertation. 


\section{Chapter 3}

\section{Matrix CFAR Detection in Riemannian Space}

Radar target detection in the presence of clutter and noise under a low signal-to-noise ratio (SNR) condition is an important and evolving problem in signal processing [71]. Specifically, the detection of small drones presents more challenging factors, such as low radar cross section, low altitude flying in cluttered environments and slow flying in the urban areas. These factors reduce the SNR and limit the number of received radar snapshots and result in a low probability of detection.

In this chapter, we focus on the problem of designing constant false alarm rate (CFAR) detectors that operate under low signal-to-clutter ratio (SCR) with limited number of snapshots. In addition, we discuss briefly the concept of the cell averaging CFAR detector which is based on the fast Fourier transform (FFT). Also, we present the CFAR detection problem in the Riemannian space where the Riemannian mean and the Riemannian distance are exploited to improve the probability of detection. Moreover, we also investigate a Kullback-Leibler detector, the Kullback-Leibler divergence and the Kullback-Leibler mean are utilized to further improve the probability of detection.

The main contributions in this chapter are two non-parametric CFAR detectors, the Riemannian-Brauer matrix and angle-based hybrid-Brauer. Both CFAR detectors exploit the Brauer disc theorem and the Riemannian and Euclidean spaces. The Riemannian-Brauer matrix and angle-based hybrid-Brauer CFAR detectors can further more enhanced the probability of detection for small sample size and low SNR.

This chapter also discusses the detection performance of the uniform linear array (ULA) in comparison with randomly spaced and slightly perturbed linear arrays. 
The randomly spaced linear array (RSLA) antenna configurations can be applicable to many applications and produce non-Toeplitz covariance matrices. In addition, slightly perturbed linear array (SPLA), which can be caused by phase noise or slight mismatch between antenna elements, produces non-Toeplitz covariance matrices. In addition, other antenna configuration, such as, circular, planar and 3D arrays may require alternative processing techniques and manifold structures. These arrays will be considered in future research.

\subsection{Cell-Averaging CFAR Detector}

A radar system's return signals may include not only target signals but also noise and clutter signals. Noise is an unwanted signal added to the desired signal and may be from various sources including, but not limited to, power supplies and amplifiers. A common statistical model for noise uses Gaussian distribution model. In addition, clutter can be defined as unwanted back-scattered signals or echoes generated from physical objects in the natural environment like ground, sea and birds. Most common clutter models are the Gaussian, the Weibull [72] and the K-distribution [73]. CFAR detection needs to adaptively adjust the detection threshold according to the background environment at a certain probability of false alarm $P_{f}$.

Cell-averaging CFAR (CA-CFAR) examines the returned signal to determine whether a target is present or absent. The interference power and the threshold are estimated by averaging the power of the cells excluding the cell under test and guards cells. Clutter and noise can cause a radar to miss detecting a real target or can increase the probability of false alarms. The probability of either of these occurrences depends on the ratio of the returned signal power to the variance of the noise level. Therefore, the SNR is a key parameter in determining the probability of detection $P_{d}$ of a target being above a specific threshold [31].

The detection threshold can be determined using random process modelling and statistical analysis of the noise, clutter and $P_{f}$. So, the returned signal $\mathbf{x}$ could have white Gaussian noise $\mathbf{n}$ plus clutter $\mathbf{c}$ only or could have a target $\mathbf{s}$ plus noise and 
clutter, then a single target detector must decide between two hypotheses

$$
\left\{\begin{array}{l}
H_{0}: \mathbf{x}=\mathbf{n}+\mathbf{c} \\
H_{1}: \mathbf{x}=\mathbf{s}+\mathbf{n}+\mathbf{c}
\end{array}\right.
$$

where the null hypothesis $H_{0}$ is background white Gaussian noise plus clutter and $H_{1}$ is a target return plus noise plus clutter. Both hypotheses can be treated as Gaussian random processes with the two probability density functions (PDF) $P_{x}\left(x \mid H_{0}\right)$ and $P_{x}\left(x \mid H_{1}\right)$ respectively.

For the case of only Gaussian noise present, the generalized likelihood ratio test (GLRT) decision rule for the returned signal $x$ can be written as

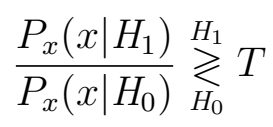

where $T$ is the detection threshold defined for a certain $P_{f}$ which can be defined as $[31]$

$$
T=N_{c}\left(P_{f}^{-1 / N_{c}}-1\right) \bar{z}
$$

where $N_{c}$ is the number of reference cells and $\bar{z}$ is the arithmetical mean of the reference cells as shown in Fig. 3.2.

Figure 3.1 illustrates the relationship between the threshold and the PDFs under the two hypotheses and shows how the $P_{d}$ and $P_{f}$ relative values depend on the relation between the two PDFs. $P_{d}$ and $P_{f}$ are the areas under the PDFs controlled by the threshold $T$. The distance between the means of the PDFs is proportional to the power of the target and is related to the SNR. The less the overlap between the PDFs the higher the $P_{d}$ and the lower the $P_{f}$. CA-CFAR detector is summarized in Fig. 3.2. Each range resolution cell $z_{i}$ is obtained from a square law detector of Doppler filter banks, where the Doppler power spectral density of the range bins of $\mathbf{x}$ is estimated by the fast Fourier transform (FFT). The CA-CFAR detector performs comparison of the cell under test $z_{D}$ with an adaptive detection threshold $T$ such that a CFAR is maintained. The adaptive detection threshold $T$ is determined by estimating the background clutter power for a desired $P_{f}$. The power of the background noise and clutter is estimated by the arithmetic mean of the reference cells around $z_{D}$ excluding 


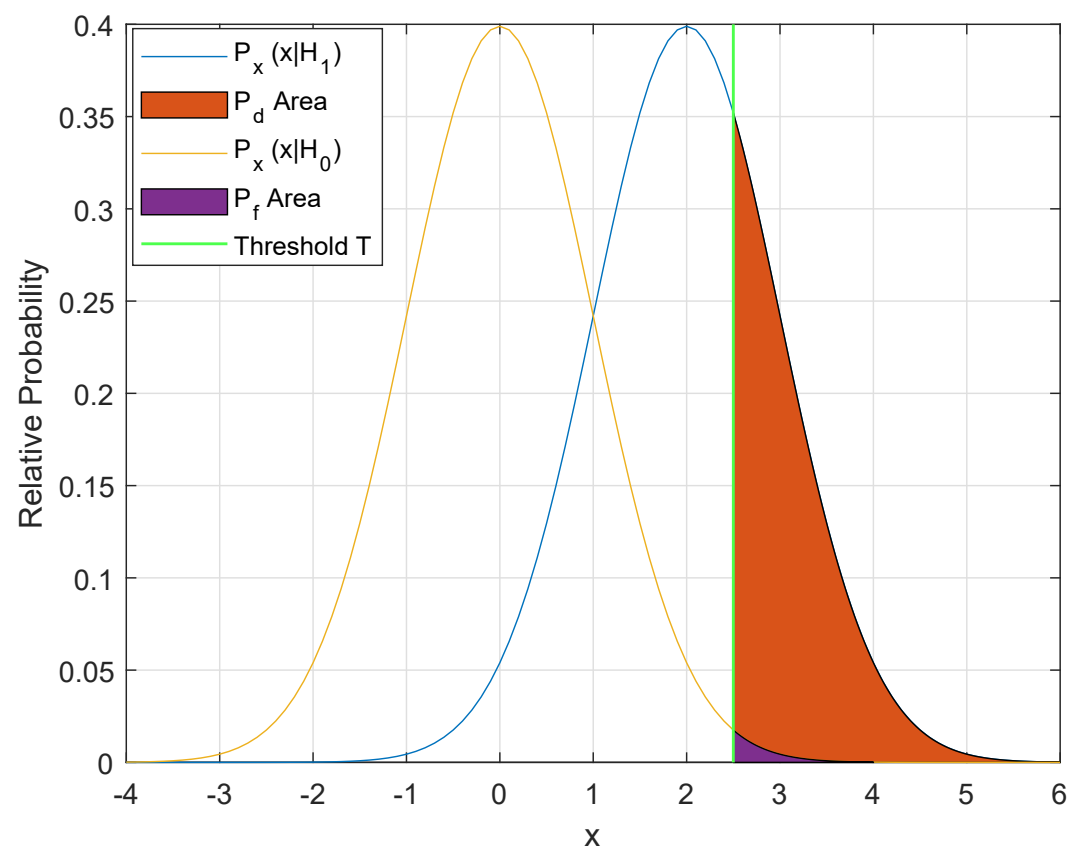

Figure 3.1: Gaussian probability density functions of noise and target.

the guard cells [74].

Although the CA-CFAR detector is easy to implement and does not require a lot of computations, it has poor Doppler resolution and suffers from performance degradation due to small number of snapshots [15]. CFAR detectors can be viewed from a geometrical point of view, where every range bin can be converted to a covariance matrix. 


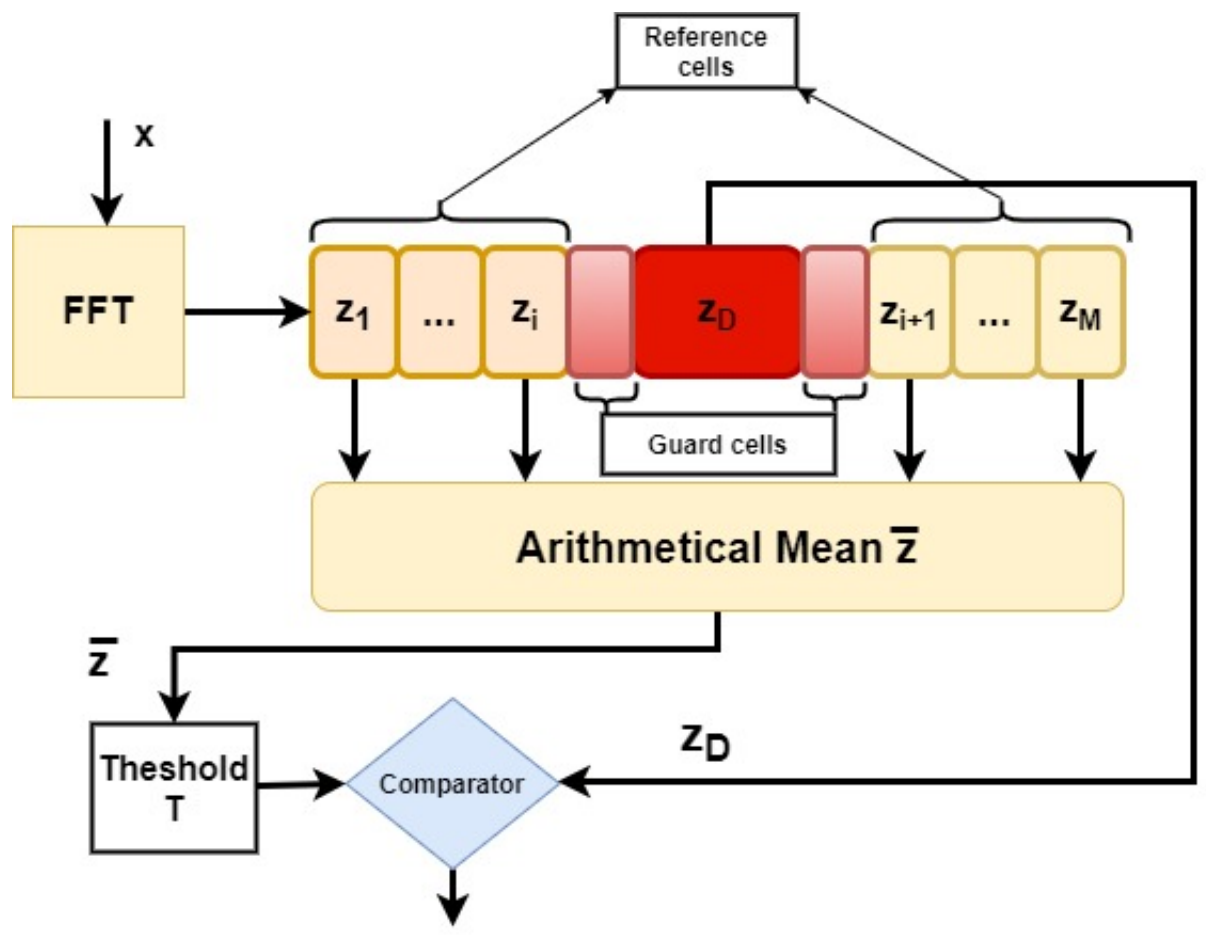

Figure 3.2: Cell averaging CFAR detector based on FFT.

\subsection{Signal Model and Detection Formulation}

The received complex radar data for an $N$ element snapshot can be written as $\mathbf{x}=$ $\left(x_{0}, \ldots, x_{N-1}\right)$ and is realized as a multivariate stationary Gaussian process with zero mean, $\mathbf{x} \sim \mathcal{C} \mathcal{N}(0, \mathbf{R})$, which can be written as

$$
P(\mathbf{x} \mid \mathbf{R})=\frac{1}{\sqrt{\pi^{N} \operatorname{det}(\mathbf{R})}} \exp \left(-\mathbf{x}^{H} \mathbf{R}^{-1} \mathbf{x}\right)
$$

where $P(\mathbf{x} \mid \mathbf{R})$ is the PDF of a circular Gaussian random vector and $\mathbf{R}$ is a THPD covariance matrix.

The hypothesis testing formulated in (3.2) can be written in terms of covariance matrices [15]

$$
\left\{\begin{array}{l}
H_{0}: \mathbf{x} \sim \mathcal{C N}\left(0, \Omega_{n}+\Omega_{c}\right) \\
H_{1}: \mathbf{x} \sim \mathcal{C N}\left(0, \Omega_{n}+\Omega_{s}+\Omega_{c}\right)
\end{array}\right.
$$

where $\Omega_{s}, \Omega_{n}$ and $\Omega_{c}$ denote the covariance matrices of the signal, noise and clutter, 
respectively. Under the null hypothesis $H_{0}$, the received snapshot consists of only noise plus clutter, and under the alternative hypothesis $H_{1}$, the received snapshot contains the target in addition to the noise and clutter.

\subsection{Riemannian Distance Based Detector}

A radar depends on reflections of its emitted energy in order to make target detection. The presence of clutter and interference sources complicate the detection process. In addition, a limited number of snapshots might add more challenges to the classical CFAR detector. The CA-CFAR detector suffers from performance degradation due to Doppler filter energy spread and poor Doppler resolution. The Riemannian distance (RD) CFAR investigates the detection problem in the space of Hermitian positivedefinite (HPD) matrices where every radar range bin (snapshot) represents a THPD covariance matrix residing on the Riemannian manifold [75]. The RD-CFAR detector is based on the information distance between the THPD covariance matrix under test and the Riemannian mean of the reference THPD covariance matrices.

\subsubsection{RD-CFAR Detector}

The RD-CFAR detector is based on the Riemannian distance $d_{R}$ between the Riemannian mean $\mathbf{R}_{r m}$ of the reference THPD covariance matrices and the THPD covariance matrix $\mathbf{R}_{\mathbf{D}}$ under test [51]. The Riemannian distance is compared with the adaptive detection threshold $T$ which can be calculated using clutter power level, the desired probability of false alarm and the parameters of the clutter model. The decision rule can be written using (2.18) and (2.21) as

$$
d_{R}\left(\mathbf{R}_{r m}, \mathbf{R}_{D}\right) \underset{H_{0}}{\stackrel{H_{1}}{\gtrless}} T .
$$

As illustrated in Fig. 3.3, the snapshot $\mathbf{x}_{i}$ is converted to THPD covariance matrix $\mathbf{R}_{i}$ and the Riemannian distance $d_{R}$ between $\mathbf{R}_{\mathbf{D}}$ and Riemannian mean $\mathbf{R}_{r m}$ is computed. The detection decision is made using (3.6) in accordance with the adaptive detection threshold $T$. For the Weibull distribution clutter model, the threshold $T$ 
can be defined as [76]

$$
T=\frac{\left(P_{f}^{-1 / N_{r}} N_{r}-1\right)^{1 / \beta}}{G\left(N_{r}, \beta\right)} \operatorname{tr}\left(\mathbf{R}_{r m}\right)
$$

where $N_{r}$ is the number of reference covariance matrices as shown in Fig. 3.3, $\beta$ is the shape parameter of the Weibull distribution, tr is the matrix trace operator, $\operatorname{tr}\left(\mathbf{R}_{r m}\right)$ is the clutter power, $G\left(N_{r}, \beta\right)=\Gamma\left(\frac{1}{\beta}+1\right)+\left(1-\Gamma\left(\frac{1}{\beta}+1\right)\right) / N_{r}^{\beta}$ and $\Gamma(\cdot)$ is the gamma function.

Although the RD-CFAR detector has better detection performance than CACFAR [77,78], it comes with added complexity. Different distance measures can be adopted in detectors and may change the detection performance. The following section will discuss the Kullback-Leibler divergence as a distance measure.

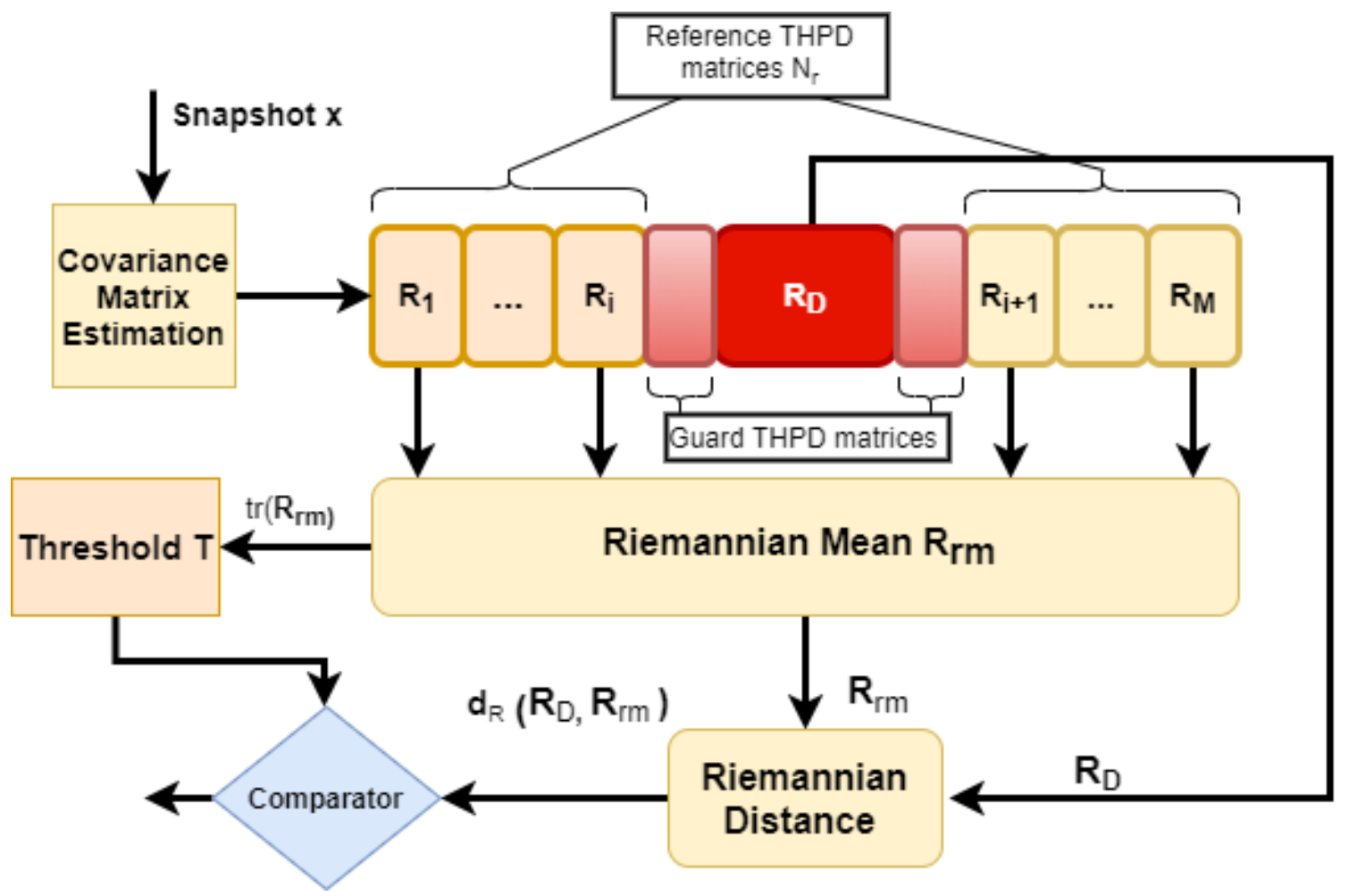

Figure 3.3: Riemannian distance CFAR detector. 


\subsection{Kullback-Leibler Detector}

The Kullback-Leibler (KLB) divergence is widely used to measure the similarity (distance) between two distributions and is also used in information theory as a measure of relative entropy [79]. In addition, the KLB divergence is a major player in the theory of statistical inference [80]. On the other hand, the KLB divergence fails to be symmetric or satisfy the triangle inequality and is not considered a genuine metric for distance as it is justified by the Pinsker-Csiszar inequality [81].

Consider $\mathbf{x}=\left(x_{0}, \ldots, x_{N-1}\right)$ are $N$ independent identically distributed observations from a statistical model whose probability density function is $q(x)$. The two hypotheses for $q(x)$ denoted by $p_{0}(x)$ and $p_{1}(x)$ are referred to as the null hypothesis and alternative hypothesis, respectively. The KLB divergence can be regarded as the expected log-likelihood ratio which can be denoted by

$$
d_{K L B}(q \| p)=\int_{-\infty}^{\infty} q(x) \ln \left(\frac{q(x)}{p(x)}\right) d x .
$$

For two multivariate Gaussian distributions parametrized by their covariance matrices, $p_{0}=\mathcal{C N}\left(\mu_{0}, \Sigma_{0}\right)$ and $p_{1}=\mathcal{C N}\left(\mu_{1}, \Sigma_{1}\right)$ and means $\mu_{0}$ and $\mu_{1}$ respectively, the KLB divergence from $p_{0}$ to $p_{1}$ can be determined using the closed form $[15,82]$

$$
d_{K L B}\left(p_{0} \| p_{1}\right)=\frac{1}{2}\left[\ln \left(\frac{\operatorname{det} \Sigma_{1}}{\operatorname{det} \Sigma_{0}}\right)+\operatorname{tr}\left(\Sigma_{1}^{-1} \Sigma_{0}\right)+\left(\mu_{1}-\mu_{0}\right)^{H} \Sigma_{1}^{-1}\left(\mu_{1}-\mu_{0}\right)-d\right] .
$$

where $d$ is the dimension of the HPD covariance matrix. The KLB mean of $M$ HPD covariance matrices can be obtained using the geometric mean of the arithmetic mean and harmonic mean of $\left\{\mathbf{R}_{1}, \mathbf{R}_{2}, \ldots, \mathbf{R}_{M}\right\}$ and can be expressed as [77,83]

$$
\mathbf{R}_{K L B}=\left(\left(\frac{1}{M} \sum_{i=1}^{M} \mathbf{R}_{i}\right)\left(\frac{1}{M} \sum_{k=1}^{M} \mathbf{R}_{k}^{-1}\right)^{-1}\right) .
$$

The detection process using the KLB divergence is illustrated in Fig. 3.4. Each snapshot is converted to THPD covariance matrix $\mathbf{R}_{i}$ using RBA in Sec. 2.4. The $\mathbf{R}_{K L B}$ is calculated using (3.10) for the reference THPD covariance matrices. The $d_{K L B}$ in (3.9) can be exploited to compute the distance between THPD covariance matrix under test $\mathbf{R}_{D}$ and $\mathbf{R}_{K L B}$. The $d_{K L B}$ is then compared to the adaptive detection threshold $T_{k}$, which is based on the clutter power, the number of reference 
covariance matrices, the parameters of the clutter model and the $P_{f}$. The detection rule can written as

$$
d_{K L B}\left(\mathbf{R}_{D}, \mathbf{R}_{\mathbf{K L B}}\right) \underset{H_{0}}{\stackrel{H_{1}}{\gtrless}} T_{k} .
$$

The threshold $T_{k}$ for Weibull distribution clutter model can be written as

$$
T_{k}=\frac{\left(P_{f}^{-1 / N_{k}} N_{k}-1\right)^{1 / \beta}}{G\left(N_{k}, \beta\right)} \operatorname{tr}\left(\mathbf{R}_{K L B}\right)
$$

where $N_{k}$ is the number of reference covariance matrices and $\operatorname{tr}\left(\mathbf{R}_{K L B}\right)$ is the clutter power.

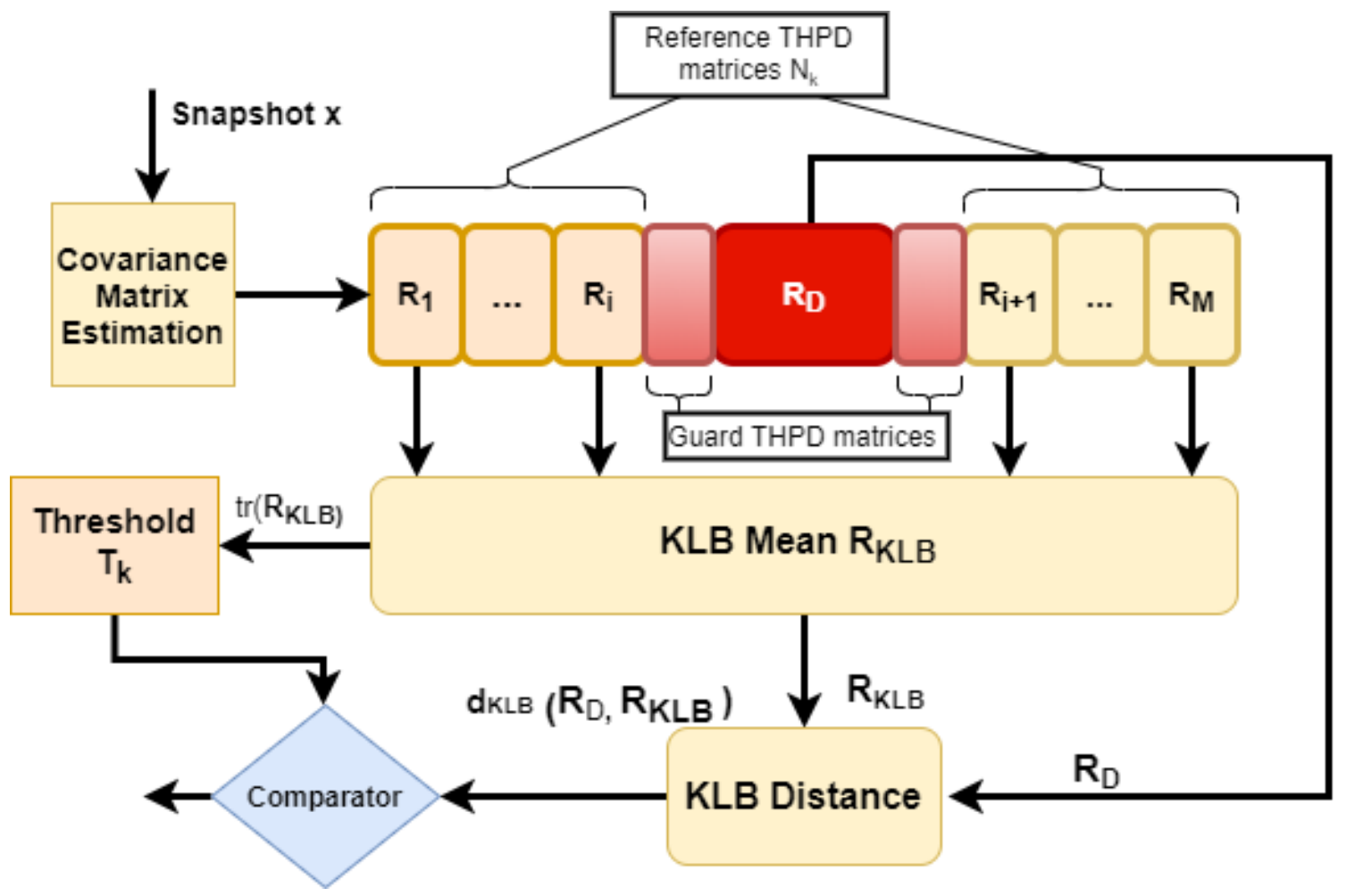

Figure 3.4: KLB based matrix CFAR detector.

The KLB-CFAR detector outperforms the CA-CFAR and RD-CFAR detectors and does not require extra computational power for computing the geometric mean in comparison with the RD-CFAR detector [78]. However, KLB-CFAR and RDCFAR detectors are not robust to outliers (THPD covariance matrices that are far 
from the Riemannian) which may affect the detection performance. The following sections will focus on designing two new CFAR detectors that are robust against outliers and require less computations to achieve better detection results.

\subsection{Riemannian-Brauer Based Detectors}

This section proposes two non-parametric CFAR detectors that exploit the Brauer disc (BD) theorem and the Riemannian space, referred to as the Riemannian-Brauer matrix (RBM) CFAR and angle-based Brauer-hybrid (ABHB) CFAR detectors, and are implemented using a two stage process. The first stage focuses on establishing a clutter-plus-noise cluster bound using the Brauer disc theorem and the second stage exploits the Riemannian and the Euclidean metrics to improve the probability of target detection.

\subsubsection{Motivation}

The radar detection of small drones in the presence of noise and clutter is considered an important task especially near sensitive places like airports or military facilities. Drones, in general, are considered as complex targets that possess a symmetrical shape with varying radar cross section. Also, the returned radar signal from drone illumination could have small sample size (limited number of snapshots) which may complicate the standard radar signal processing. Hence, robust and reliable CFAR detectors that successfully detect drones in cluttered and noisy environments with limited number of snapshots are much needed to undertake countermeasures.

Classical detection schemes like CA-CFAR utilize the FFT to obtain the correlation of sample data. This CFAR technique performs well for a large sample size data [31]. However, for small number of snapshots, the CA-CFAR detector suffers from performance degradation and poor Doppler resolution [15]. [51] proposed the RD-CFAR detector to overcome these drawbacks by projecting the detection problem into the Riemannian space of HPD matrices. The RD-CFAR detector exploits the Riemannian mean of the HPD covariance matrices and the Riemannian distance between the Riemannian mean and the HPD covariance matrix under test to improve the detection performance. The KLB-CFAR is similar in process to the RD-CFAR, but the KLB mean and the KLB divergence are used instead of the Riemannian mean and the Riemannian distance. Unlike the Riemannian mean, the KLB mean is based 
on the geometric mean of the arithmetic mean and the harmonic mean of the HPD matrices, which require less computation. However, the RD-CFAR and the KLBCFAR have two major drawbacks. First, the number of Riemannian mean and KLB mean computations are proportional to the number of snapshots, which are computationally expensive due to the matrix exponential, logarithm and the geometric mean calculations. Second, the Riemannian mean and the KLB mean should be potential targets (outliers) free, however they are not robust to outliers [77].

The eigenvalues of covariance matrices can be a good indicator of desired signal and noise. In addition, eigenvalues play a significant role in determining Riemannian distance between two points on the Riemannian manifold. Motivated by the importance of the eigenvalues and their inclusion regions, the drawbacks of RD-CFAR and the KLB-CFAR can be tackled by establishing inclusion cluster bounds around the clutter-plus-noise and potential targets eigenvalues using the Brauer disc theorem.

Two non-parametric CFAR detectors, the Riemannian-Brauer matrix (RBM) CFAR and angle-based hybrid-Brauer (ABHB) CFAR detectors are proposed to overcome the challenges in the RD-CFAR and the KLB-CFAR. The RBM-CFAR and ABHB-CFAR detectors explore the idea of utilizing the Brauer disc theorem to establish a cluster bound around the clutter-plus-noise covariance matrices by examining the maximum eigenvalue for each of the THPD covariance matrices residing on the Riemannian manifold. Also, the Riemannian distance computations are proportional to the number of outliers and the Riemannian mean of the clutter-plus-noise covariance matrices is computed only once.

\subsubsection{Brauer Cluster Bound}

The Brauer disc theorem 2.7.2 can be exploited to establish a cluster bound around the maximum eigenvalues of the $N$ THPD covariance matrices. The maximum eigenvalues of the $N$ THPD covariance matrices are bounded by the largest BDs. The centre of the largest BD $c_{i}$ for each THPD covariance matrix, which is the inclusion region for the maximum eigenvalue, represents the power of the snapshot $P_{0}$ as shown in Algorithm 1. In addition, the minimum BD of the maximum eigenvalues of the $N$ clutter-plus-noise THPD covariance matrices represents the minimum bound with radius $\rho$ as shown in Fig 3.5. Utilizing the BD centres of the $N$ THPD covariance matrices, the Brauer cluster bound $T_{B}$ for the clutter-plus-noise can be established 
as

$$
T_{B}=\frac{\frac{1}{M} \sum_{i=1}^{M} c_{i}}{\left(\prod_{i=1}^{M} c_{i}\right)^{\frac{1}{M}}} \rho
$$

where $\frac{1}{M} \sum_{i=1}^{M} c_{i}$ and $\left(\prod_{i=1}^{M} c_{i}\right)^{\frac{1}{M}}$ are the arithmetic mean and geometric mean of the centres of the Brauer discs, respectively. The THPD covariance matrices corresponding to the eigenvalues inside the Brauer cluster bound are considered clutter-plus-noise covariance matrices. On the other hand, the THPD covariance matrices associated with the eigenvalues outside the Brauer cluster bound are considered THPD matrices with potential targets. As an example, consider a radar received signal of 8 snapshots. Each snapshot is converted to a THPD covariance matrix and the BD for the maximum eigenvalues of the THPD covariance matrices with (3.13) can be used to establish a Brauer cluster bound as shown in Fig. 3.5. The THPD covariance matrices associated with the eigenvalues outside the Brauer cluster bound are considered as the potential targets.

\subsubsection{Riemannian-Brauer Matrix CFAR Detector}

The RBM-CFAR detector is a two stage process based on the Riemannian distance between the Riemannian mean and the potential targets' THPD covariance matrices. The first stage utilizes the RBA to convert each range cell into a THPD covariance matrix and exploits the BD theorem to cluster the maximum eigenvalues of the THPD covariance matrices. By using (3.13), a clutter-plus-noise cluster bound and a potential target bound of the THPD covariance matrices can be established. The second stage involves computing the Riemannian distance $d_{R B}$ between the Riemannian mean of the clutter-plus-noise THPD covariance matrices and the potential target THPD covariance matrices. The rule of detection for the RBM-CFAR detector can be formulated using (2.21), (2.18) and (3.13) as

$$
d_{R B}\left(\mathbf{R}_{r m}\left(T_{B}\right), \mathbf{R}_{T_{i}}\right) \underset{H_{0}}{\stackrel{H_{1}}{\gtrless}} \delta
$$




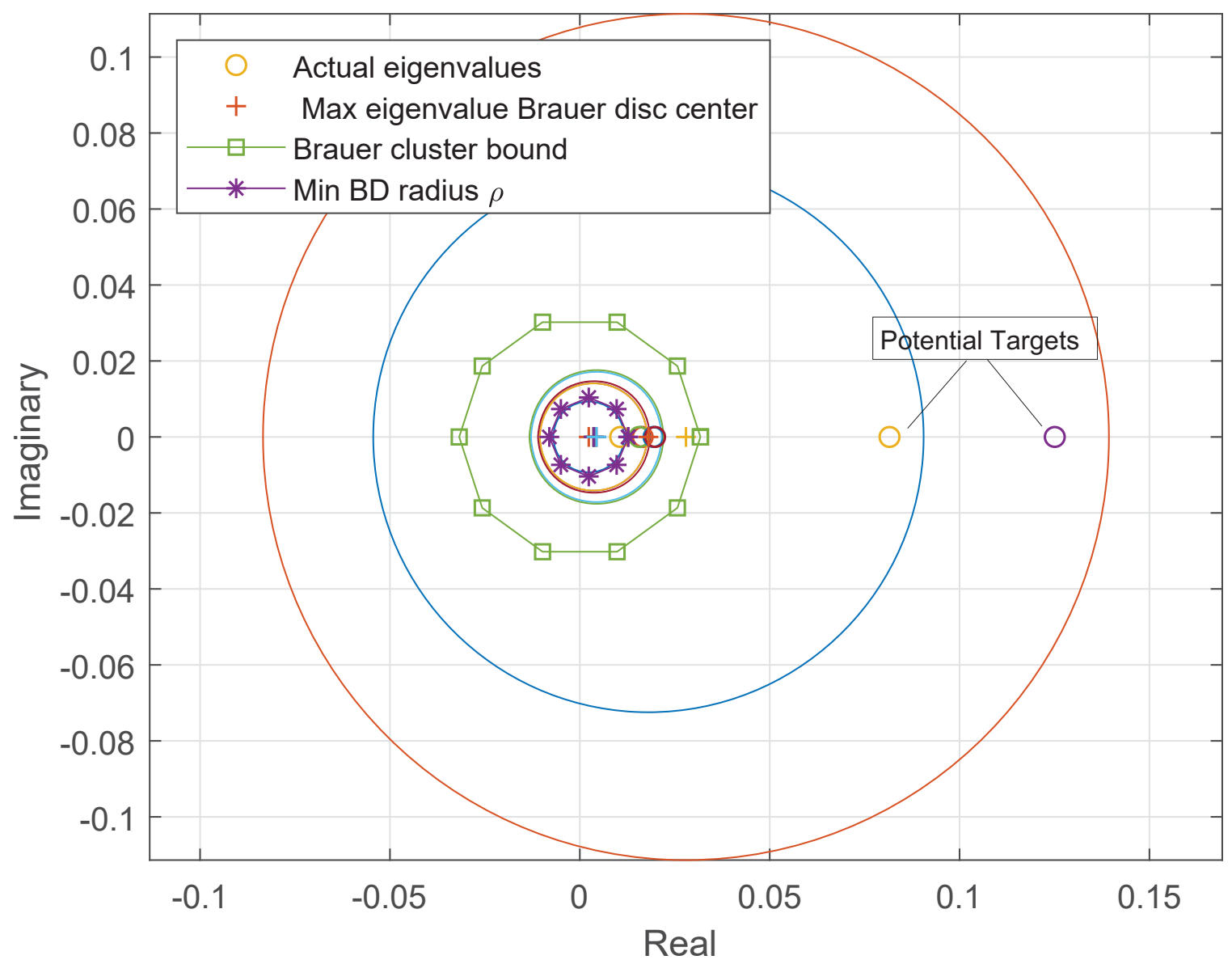

Figure 3.5: Example of Brauer cluster bound for clutter-plus-noise eigenvalues and maximum eigenvalues of potential targets THPD covariance matrices with their associated Brauer discs.

where $\mathbf{R}_{r m}\left(T_{B}\right)$ is the Riemannian mean of the THPD covariance matrix inside the Brauer cluster bound, $\mathbf{R}_{T_{i}}$ is the THPD covariance matrix of a potential target and $\delta$ is an adaptive threshold. $\delta$ is based on the probability of false alarm $P_{f}$ and noise or clutter power levels obtained from the $\mathbf{R}_{r m}$ of the clutter-plus-noise covariance matrices, which can be written as

$$
\delta=-\operatorname{tr}\left(\mathbf{R}_{r m}\right) \ln \left(P_{f}\right)
$$

The RBM-CFAR detection process is illustrated in Fig. 3.6. The first step of the RBM-CFAR detector is converting all of the snapshots into THPD covariance matrices using RBA from Sec. 2.4 and applying the BD theorem 2.7.2 to cluster 


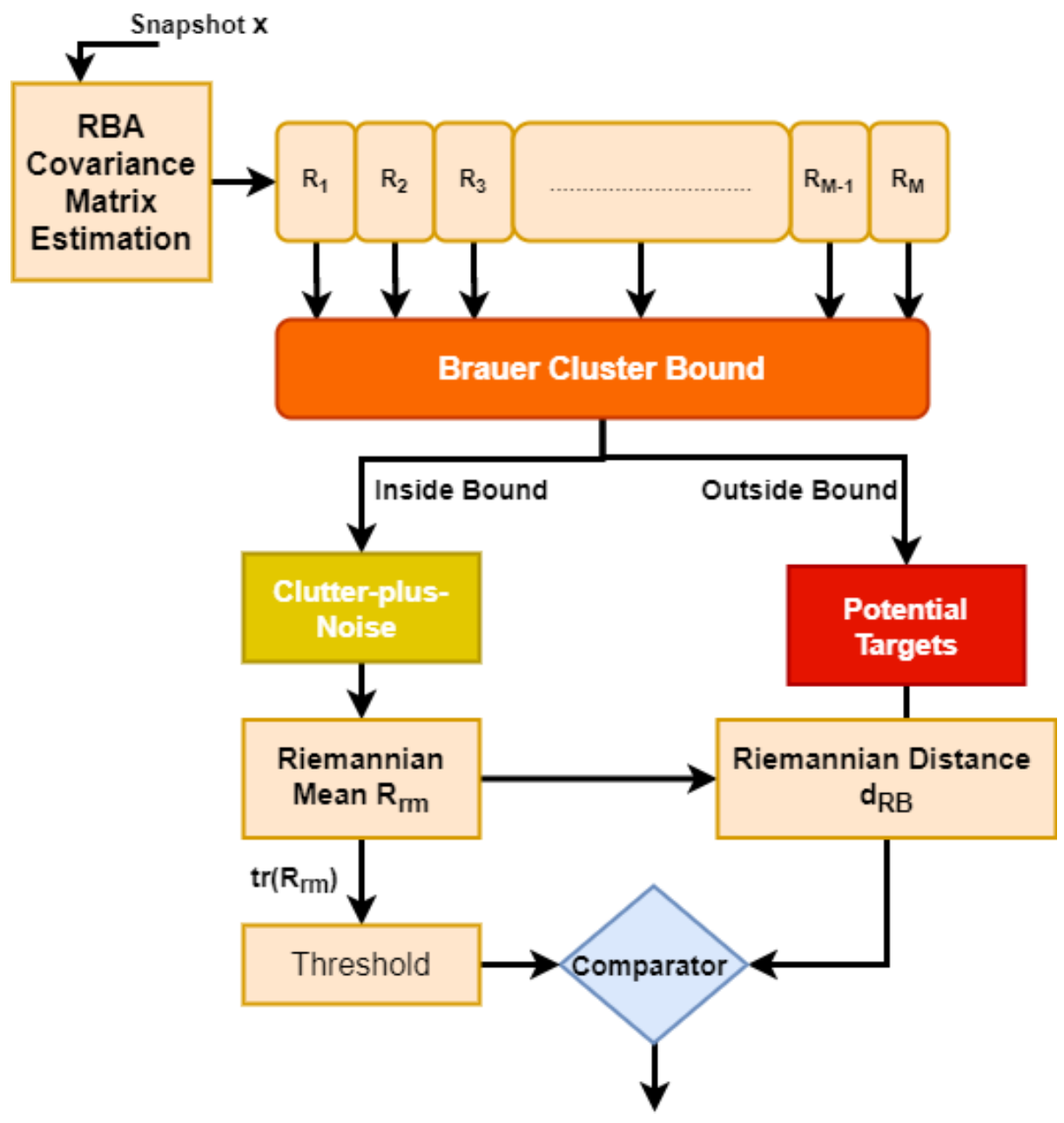

Figure 3.6: RBM-CFAR detector.

the THPD covariance matrices of the the clutter-plus-noise and identify the THPD covariance matrices of the potential targets outside the cluster bound. Then, we calculate the Riemannian mean of the clutter-plus-noise covariance matrices and the Riemannian distance between the Riemannian mean and the potential targets. The calculated Riemannian distance is compared to the threshold $\delta$.

The RBM-CFAR detector has many advantages over RD-CFAR detector [51]. First, the computations of the Riemannian mean and the median do not include any potential targets since the principle eigenvalues of the THPD covariance matrix of the potential targets are larger than the eigenvalues of the clutter-plus-noise THPD covariance matrices; hence, the Riemannian mean and the Riemannian median are 


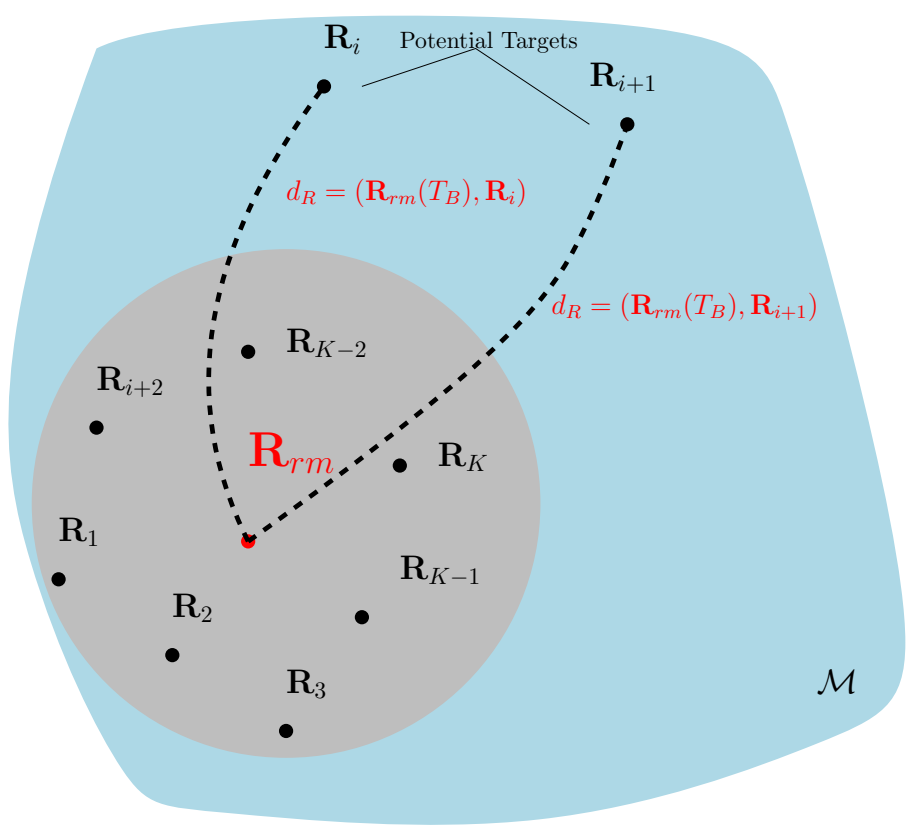

Figure 3.7: Clutter-plus-noise THPD covariance matrices cluster bound and THPD covariance matrices of potential targets.

robust to outliers. Second, the computational cost is less since the Riemannian mean is only calculated once as opposed to the RD-CFAR as shown in Figures 3.3 and 3.6. The Riemannian distance calculation depends on the number of outliers as shown in Fig. 3.7.

\subsubsection{Angle Based Hybrid-Brauer CFAR Detector}

The ABHB-CFAR detector exploits the Euclidean tangent space and the Riemannian geodesical distances between points on the Riemannian manifold. In addition, it utilizes the Riemannian mean of the clutter-plus-noise cluster bound $\mathbf{R}_{r m}$, the Riemannian median of the clutter-plus-noise cluster bound $\mathbf{R}_{r m e d}$ and projection of both the $\mathbf{R}_{r m}$ and $\mathbf{R}_{r m e d}$ on the tangent space of the potential target point. The triangle connecting the $\mathbf{R}_{r m}, \mathbf{R}_{r m e d}$ and potential target on the Riemannian manifold can generate a geodesic triangle in which the law of cosines can be used [84] as shown in Fig 3.8.

Recall if $a, b, c$ lie in Euclidean space, the law of cosines states that

$$
\|b-c\|^{2}=\|b-a\|^{2}+\|c-a\|^{2}-2\langle b-a, c-a\rangle
$$




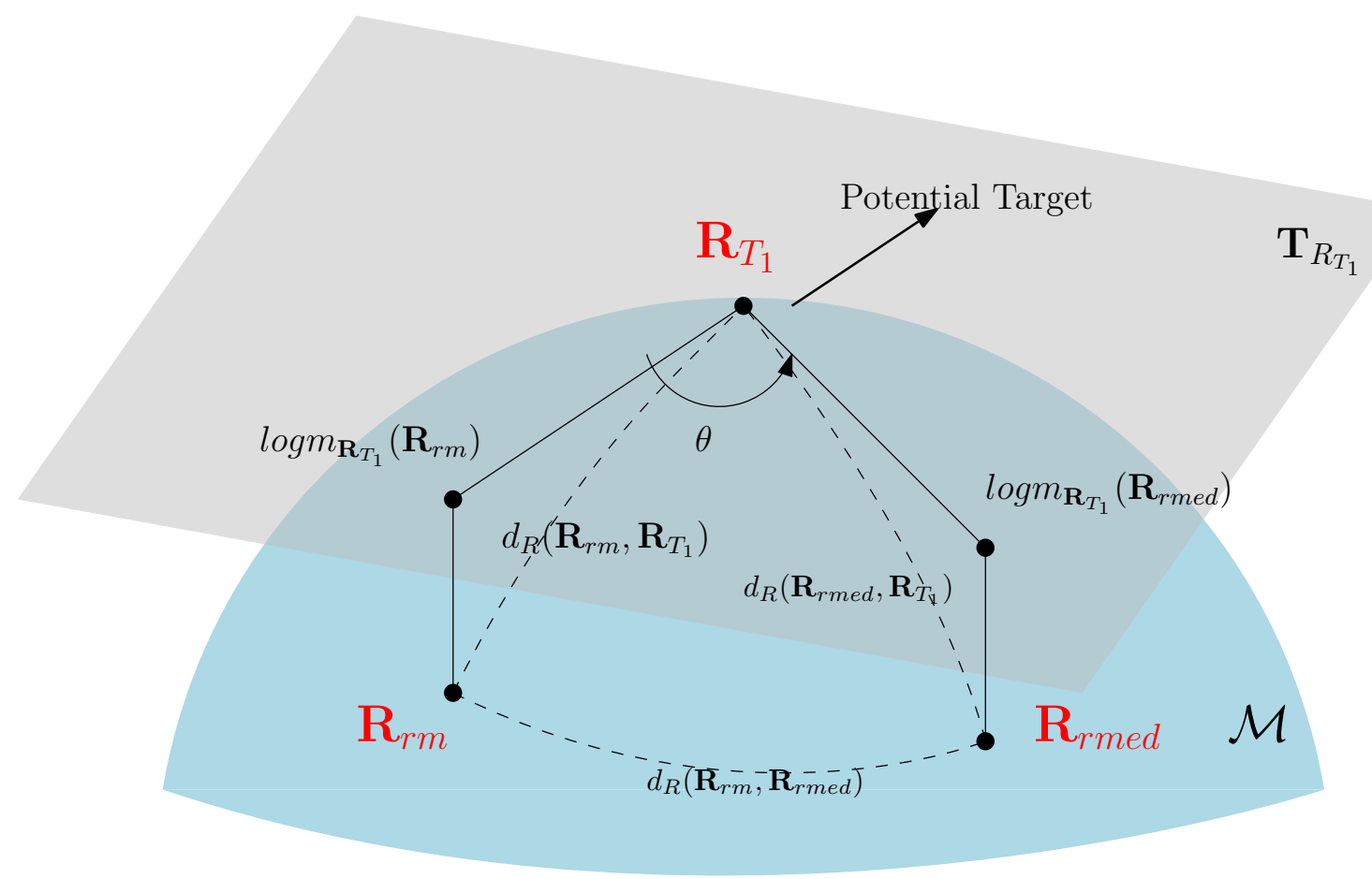

Figure 3.8: Geodesical triangle showing the angle at potential target on the manifold.

where $\|\cdot\|$ is the magnitude and $\langle\cdot, \cdot\rangle$ represents the dot product. The law of cosines can be applied to the geodesic triangle on the Riemannian manifold where the vertices of the triangle are $\mathbf{R}_{r m}, \mathbf{R}_{r m e d}$ and potential target point $\mathbf{R}_{T_{i}}$. Using (2.21), (2.22), (2.14), (2.18), (3.13) and (3.16), the law of cosines on manifold can be formulated as $\left\|d_{R}\left(\mathbf{R}_{r m}, \mathbf{R}_{r m e d}\right)\right\|^{2}=\left\|d_{R}\left(\mathbf{R}_{r m}, \mathbf{R}_{T_{i}}\right)\right\|^{2}+\left\|d_{R}\left(\mathbf{R}_{r m e d}, \mathbf{R}_{T_{i}}\right)\right\|^{2}-2\left\langle\log \mathrm{R}_{\mathbf{R}_{i}}\left(\mathbf{R}_{r m}\right), \log \mathrm{R}_{\mathbf{R}_{T_{i}}}\left(\mathbf{R}_{r m e d}\right)\right\rangle_{\mathbf{R}_{T_{i}}}$

where $\log \mathbf{R}_{\mathbf{R}_{i}}\left(\mathbf{R}_{r m}\right)$ is the projection of $\mathbf{R}_{r m}$ onto the tangent space of $\mathbf{R}_{T_{i}}$. The dot product term can be written in terms of angle

$$
\left\langle\operatorname{logm}_{\mathbf{R}_{T_{i}}}\left(\mathbf{R}_{r m}\right), \log \mathrm{R}_{\mathbf{R}_{T_{i}}}\left(\mathbf{R}_{r m e d}\right)\right\rangle_{\mathbf{R}_{T_{i}}}=\left\|\log \mathrm{m}_{\mathbf{R}_{T_{i}}}\left(\mathbf{R}_{r m}\right)\right\|\left\|\log \mathrm{m}_{\mathbf{R}_{T_{i}}}\left(\mathbf{R}_{r m e d}\right)\right\| \cos (\theta)
$$


where $\theta$ is the angle at point $\mathbf{R}_{T_{i}}$ on the tangent space which can be written as

$$
\cos (\theta)=\frac{\left\|d_{R}\left(\mathbf{R}_{r m}, \mathbf{R}_{T_{i}}\right)\right\|^{2}+\left\|d_{R}\left(\mathbf{R}_{r m e d}, \mathbf{R}_{T_{i}}\right)\right\|^{2}-\left\|d_{R}\left(\mathbf{R}_{r m}, \mathbf{R}_{r m e d}\right)\right\|^{2}}{2\left\|\operatorname{logm}_{\mathbf{R}_{T_{i}}}\left(\mathbf{R}_{r m}\right)\right\|\left\|\operatorname{logm}_{\mathbf{R}_{T_{i}}}\left(\mathbf{R}_{r m e d}\right)\right\|}
$$

The rule of detection for the ABHB CFAR detector can be formulated using (3.19) as

$$
\theta \underset{H_{1}}{\stackrel{H_{0}}{\gtrless}} \gamma
$$

where $\gamma$ is an adaptive threshold angle of detection which can be formulated using (3.15), (3.17) and the Riemannian median threshold $-\operatorname{tr}\left(\mathbf{R}_{r m e d}\right) \ln \left(P_{f}\right)$ as

$$
\gamma=\operatorname{acos}\left(\frac{\left\|-\operatorname{tr}\left(\mathbf{R}_{r m}\right) \ln \left(P_{f}\right)\right\|^{2}+\left\|-\operatorname{tr}\left(\mathbf{R}_{r m e d}\right) \ln \left(P_{f}\right)\right\|^{2}-\left\|d_{R}\left(\mathbf{R}_{r m}, \mathbf{R}_{r m e d}\right)\right\|^{2}}{2\left\|-\operatorname{tr}\left(\mathbf{R}_{r m}\right) \ln \left(P_{f}\right)\right\|\left\|-\operatorname{tr}\left(\mathbf{R}_{r m e d}\right) \ln \left(P_{f}\right)\right\|}\right)
$$

The farther the potential target point, the smaller the angle on the manifold. The ABHB-CFAR detector is illustrated in Fig. 3.9. All snapshots are converted to THPD covariance matrices and the BD theorem 2.7.2 is applied to cluster the THPD covariance matrices into two bounds: clutter-plus-noise bound and potential targets bound. For every potential target, the law of cosines is applied using the Riemannian distances between the Riemannian mean, the Riemannian median and the potential target. The calculated angle at the potential target on the Riemannian manifold is compared with angle threshold $\gamma$. 


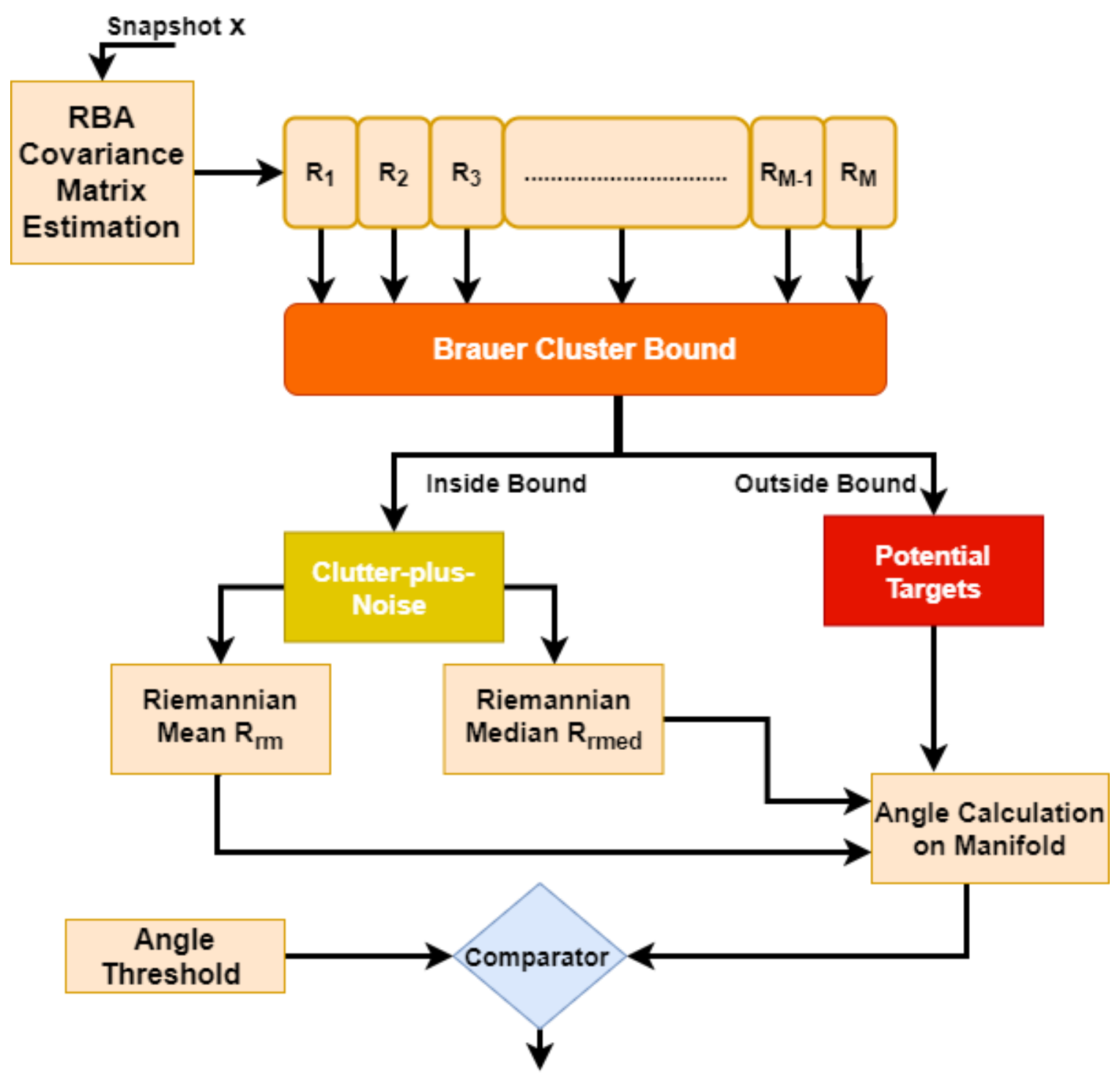

Figure 3.9: ABHB-CFAR detector. 


\subsection{Simulations Results}

The simulation results in this section consist of three parts: evaluate the performance of the RBM-CFAR and ABHB-CFAR techniques in comparison with CA-CFAR, RDCFAR and KLB-CFAR detectors via Monte Carlo simulations, test the CFAR property of the proposed CFAR detectors and investigate the effect of ULA versus RSLA configurations on the detection process. A collocated directional ULA is used with $N=8$ receivers and the antenna elements spaced half a wavelength $\lambda$ apart from each other as shown in Fig. 3.10. Also, a collocated directional RSLA with 8 antenna elements spaced randomly as shown in Fig. 3.11 is also considered in the simulation. The snapshots are generated from a moving target with a velocity of $v=10 \mathrm{~m} / \mathrm{s}$ away from the radar. Every snapshot (range bin) is converted to a THPD covariance using the RBA. The RBA regularization parameter is set to $\psi_{1}=0.01$ and the autoregressive order model is $N=8$. Also, the Riemannian mean step size is $\epsilon=0.01$ and the number of iterations is set to $t=1000$ with tolerance level set to $10^{-6}$.

The first part of the simulations is based on comparing the detection performance of the proposed CFAR detector with the CA-CFAR, the RD-CFAR and the KLBCFAR CFAR detectors. The data is generated from 5 received pulses and $M=25$ snapshots and the target is located at the $10^{\text {th }}$ range bin. The clutter model is based on a Weibull distribution with scale parameter $\alpha=1$ and shape parameter $\beta=3$. The covariance matrix model $\Sigma_{d}$ for generating the snapshots can be defined as [72]

$$
\Sigma_{d}(i, k)=\sigma_{c}^{2} \rho^{|i-k|} \exp \left(j 2 \pi \frac{v}{\lambda}(i-k)\right) i, k=1, \ldots, N
$$

where $\sigma_{c}$ is the clutter power, $\rho$ is the one lag correlation coefficient and $\lambda$ is the wavelength. In this simulation, $\rho=0.9$ and $\lambda=1 \mathrm{~m}$. For CA-CFAR, the number of cells that are averaged is $Q=10$ and for the RD-CFAR and the KLB-CFAR, the number of THPD covariance matrices averaged is $Q=10$. The RBM-CFAR and the ABHB-CFAR Riemannian mean is based on the THPD covariance matrices inside the BD cluster bound, which is not biased to any outlier. The covariance matrix model for the RD-CFAR, the KLB-CFAR, the RBM-CFAR and the ABHB-CFAR is based on (2.11).

The detection threshold for the Weibull clutter model is calculated using (3.7) 


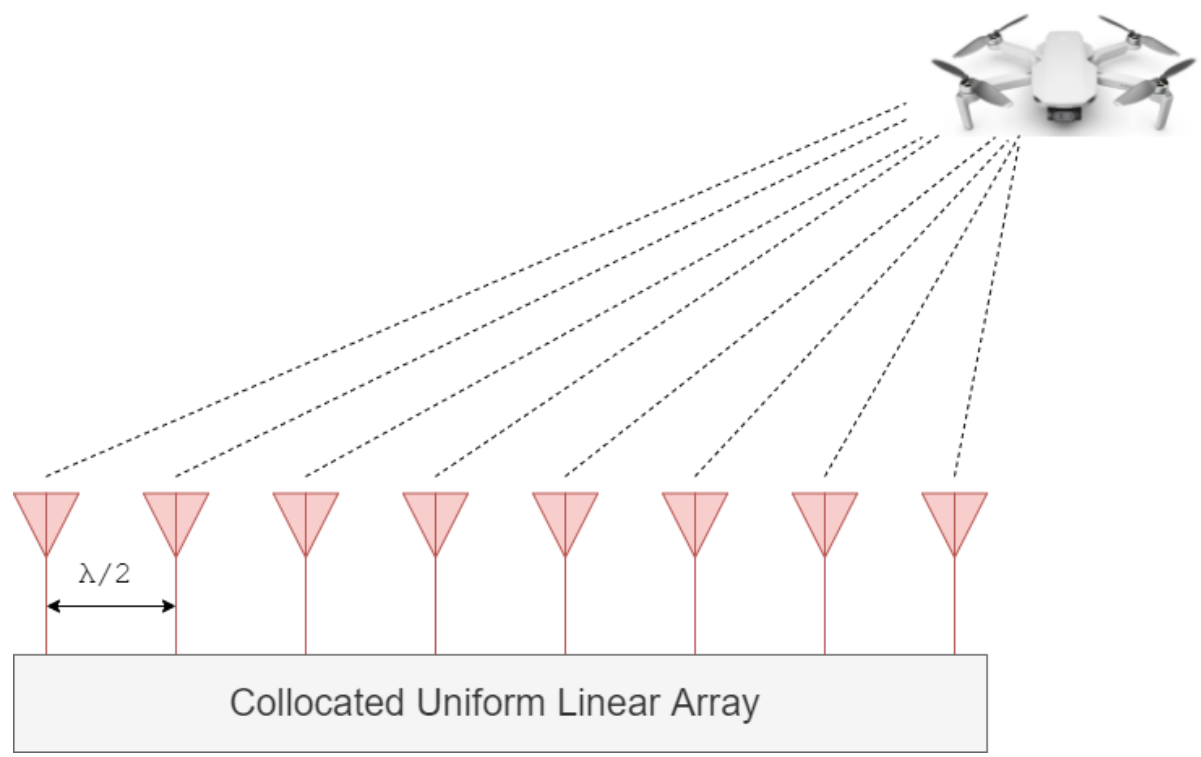

Figure 3.10: Typical collocated $8 \times 8$ ULA antenna system with a drone target.

and (3.12). The simulation is based on 20000 Monte Carlo runs to determine the probability of detection $P_{d}$ and the detection threshold. The performance of all five CFAR detectors for different $P_{f}$ are shown in Fig. 3.12. We observe that at high $P_{f}=10^{-3}$, all the CFAR techniques except the RD-CFAR require less than $5 \mathrm{~dB}$ SCR to be able to detect a target. Also, at low $P_{f}=10^{-6}$, only the RBM-CFAR and ABHB-CFAR require less than $5 \mathrm{~dB}$ SCR for target detection. In addition, the simulation results demonstrate that both RD-CFAR and KLB-CFAR outperform the CA-CFAR at high and low $P_{f}$. Furthermore, the performance of the RBM-CFAR and ABHB-CFAR is better than the RD-CFAR and the KLB-CFAR with at least $2-5 \mathrm{~dB}$ signal-to-clutter improvement at high and low $P_{f}$.

The second part of the simulations examines the CFAR property of the RBM and ABHB CFAR detectors. According to the CFAR property, the performance of the detector must not change if we scale the signal and noise. The data generated using ULA configuration and a target was placed in the $4^{\text {th }}$ range bin. This simulation is based on 1000 Monte Carlo runs to determine the probability of detection $P_{d}$ for constant false alarm $P_{f}=10^{-4}$. The signal and noise scaling factors are 10 and 50 . As shown in Fig. 3.13, we notice a slight variation between the original detection curve and the scaled detection curves for both CFAR detectors. This is due to the computation of the Riemannian mean, which is an iterative gradient algorithm (2.21). 


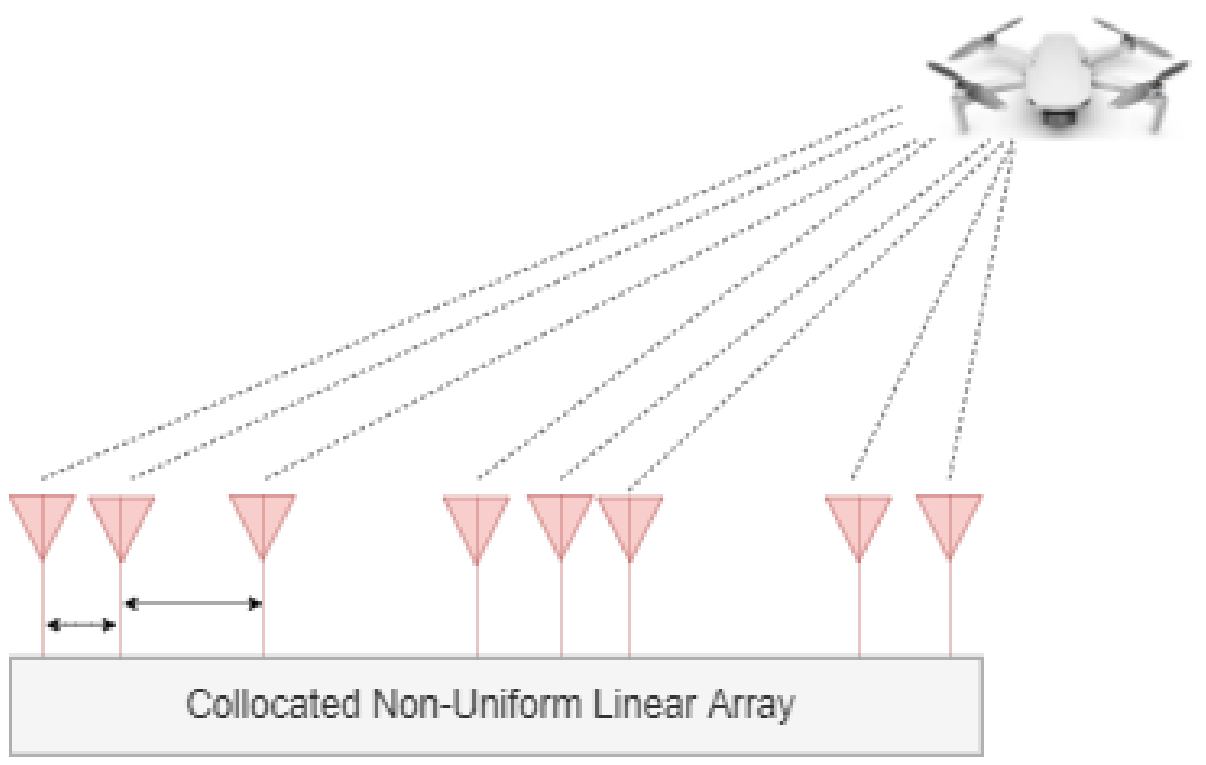

Figure 3.11: Typical collocated $8 \times 8$ RSLA antenna system with internal antennas spacing $[0.3 \lambda, 0.9 \lambda, 1.75 \lambda, 0.25 \lambda, 0.15 \lambda, 2.1 \lambda, 0.4 \lambda]$ and drone target.

The performance of the RBM an ABHB CFAR detector satisfy the CFAR property while maintaining a constant false alarm.

The third part of the simulations studies the influence of RSLA and SPLA on the detection process. The target was placed at the $9^{\text {th }}$ bin. The Toeplitz structure of the sample covariance matrices are usually generated from a ULA configuration. Other array configurations, such as RSLA configuration, may generate non-Toeplitz covariance matrices, which may affect the target detection performance in the Riemannian space. The RBA estimates a THPD covariance matrix for every snapshot regardless of the antenna configuration, however by varying the spacing between internal antenna elements while keeping the same size array has an impact on the propagation delay between antennas which affects the eigenvalues of the estimated covariance matrices. Hence, the Riemannian distance between THPD covariance matrices may change. Figure 3.14 shows the Brauer bound for clutter-plus-noise and potential target at the $9^{\text {th }}$ range bin for ULA and RSLA configurations. The Brauer disc and the eigenvalue of the target for the ULA are larger than the RSLA due to random spacing between antenna elements. Also, the RBM and ABHB CFAR detectors for ULA and RSLA maintained a good detection for 16 snapshots at $5 \mathrm{~dB}$ SNR, as shown in Fig. 3.15. In addition, the performance of the RBM and ABHB CFAR detectors are compared 
with the CA-CFAR, the RD-CFAR and the KLB-CFAR at 5 dB SNR for ULA and RSLA configurations as shown in Figs. 3.16 and 3.17. All techniques detected the target at the $9^{\text {th }}$ range bin for both ULA and RSLA configurations.

The performance of the proposed CFAR detectors under ULA, SPLA and RSLA configurations can be investigated by varying the SNR of the data and maintaining a constant $P_{f}$ for 16 snapshots pulses. Figure 3.18 illustrates the detection performance at $P_{f}=10^{-4}$. We notice that at higher SNR, the ABHB and the RBM CFAR detectors under the slight perturbed ULA loses around $1 \mathrm{~dB}$ and under the RSLA loses around $2.5 \mathrm{~dB}$ in comparison with ULA configuration. On the other hand, at low SNR, the ABHB and RBM CFAR detectors under ULA, SPLA and RSLA maintained the same performance. Figure. 3.19 shows the distribution of averaged $P_{d}$ over 2500 pulses of 16 snapshots for ULA, SPLA and RSLA configurations for RBM and ABHB CFAR detectors respectively. We can see that the median $P_{d}$ for ULA is higher than the SPLA and RSLA and the SPLA is higher than the RSLA. The RBA guarantees Toeplitz structure regardless of the array configuration, however the RSLA and the SPLA affect the eigenvalues of the THPD covariance matrices; hence, their performances are degraded in comparison with the ULA configuration. In addition, ULA, RSLA and SPLA suffer from ambiguities caused by the direction of arrival of the targets and by increasing the spacing amongst the antenna elements. Some direction of arrival angles may cause linear dependency amongst the steering vectors, which may generate ambiguous errors and lead to poor detection performance. Also, increasing the spacing between antenna elements to more than half-wavelength may generate grating lobes and high sidelobe levels, which may mask the targets and lead to degraded detection performance. 


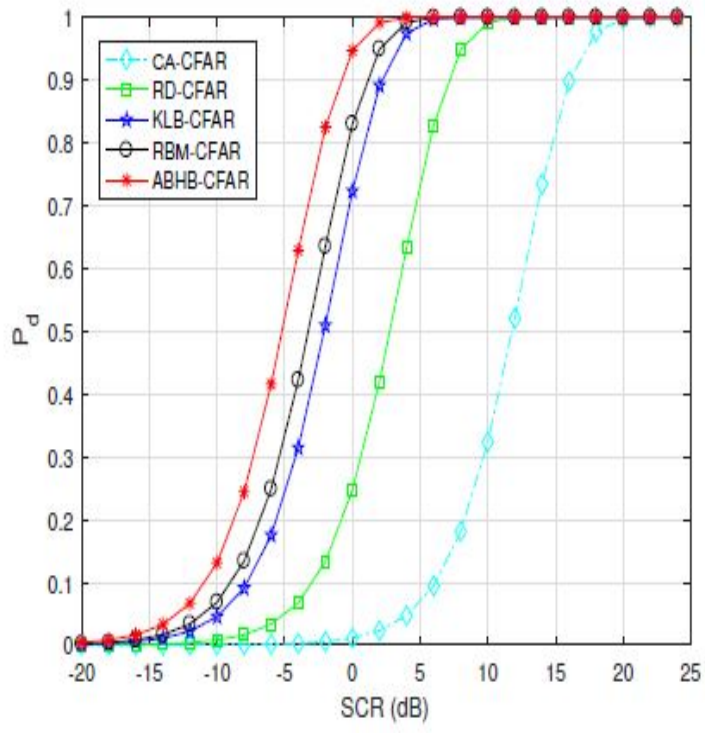

(a) $P_{f}=10^{-3}$

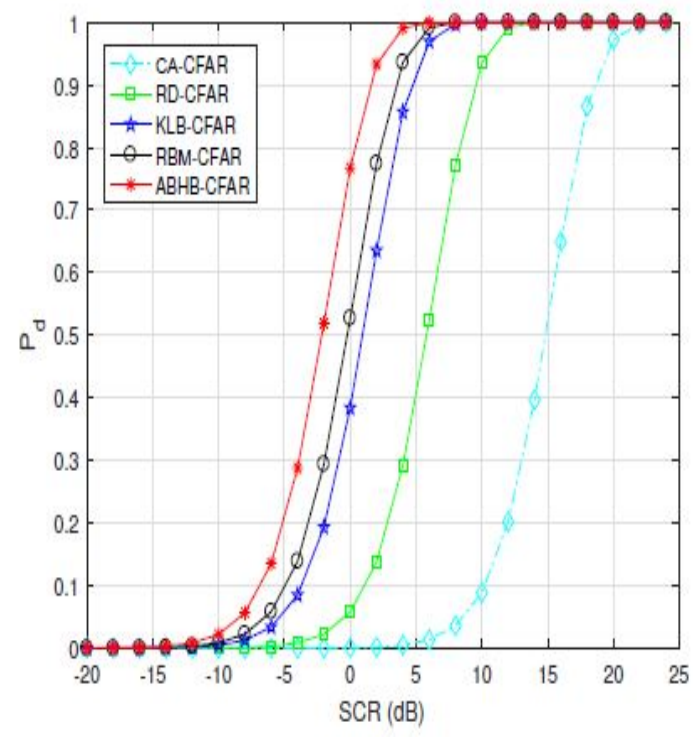

(c) $P_{f}=10^{-5}$

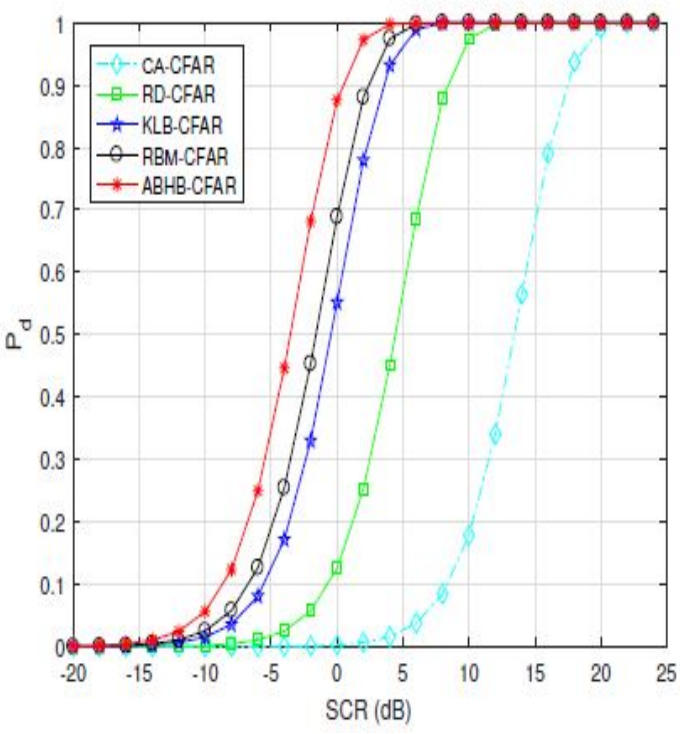

(b) $P_{f}=10^{-4}$

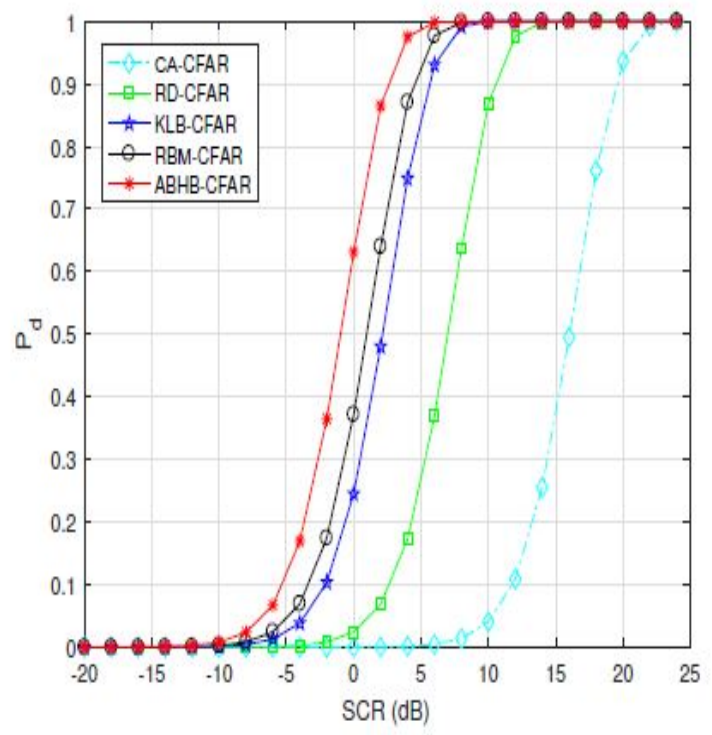

(d) $P_{f}=10^{-6}$

Figure 3.12: Performance comparison of the CA-CFAR, RD-CFAR, KLB-CFAR, RMB-CFAR and ABHB-CFAR detectors with different $P_{f}$. 


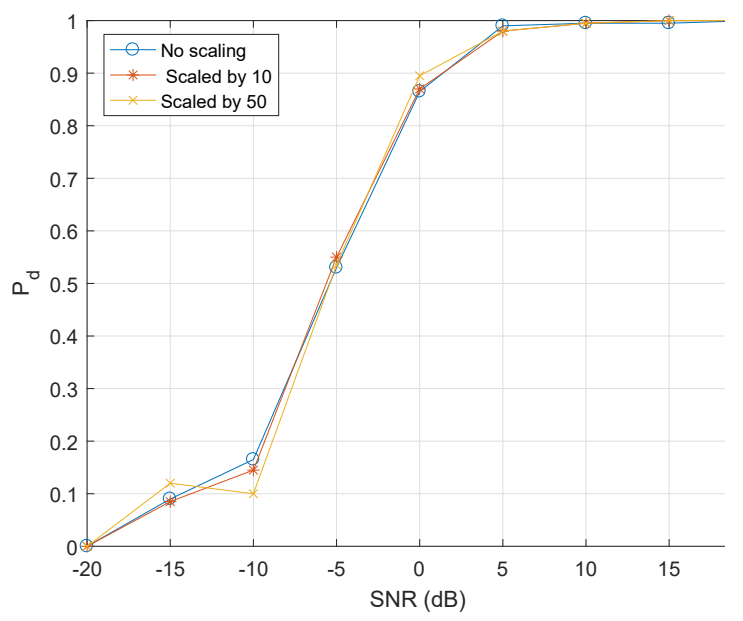

(a) ABHB-CFAR detector

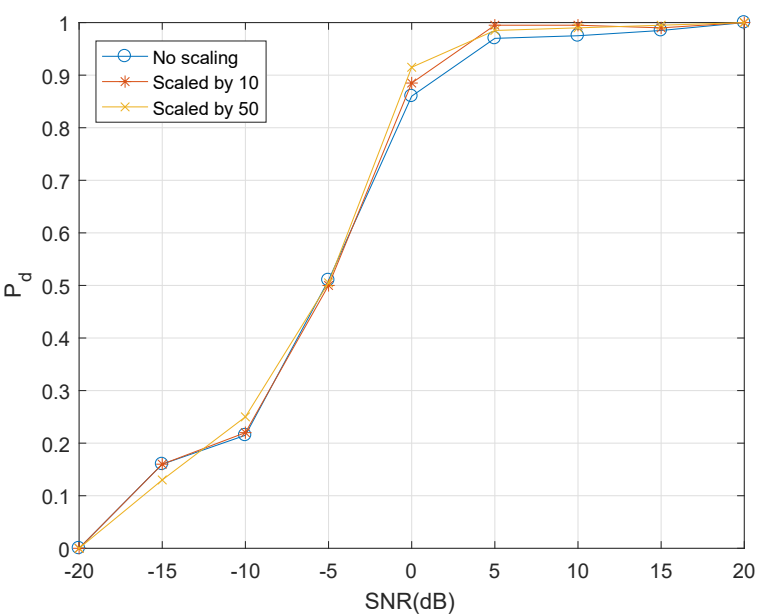

(b) RBM-CFAR detector

Figure 3.13: Detection performance curves for original and scaled signal and noise.

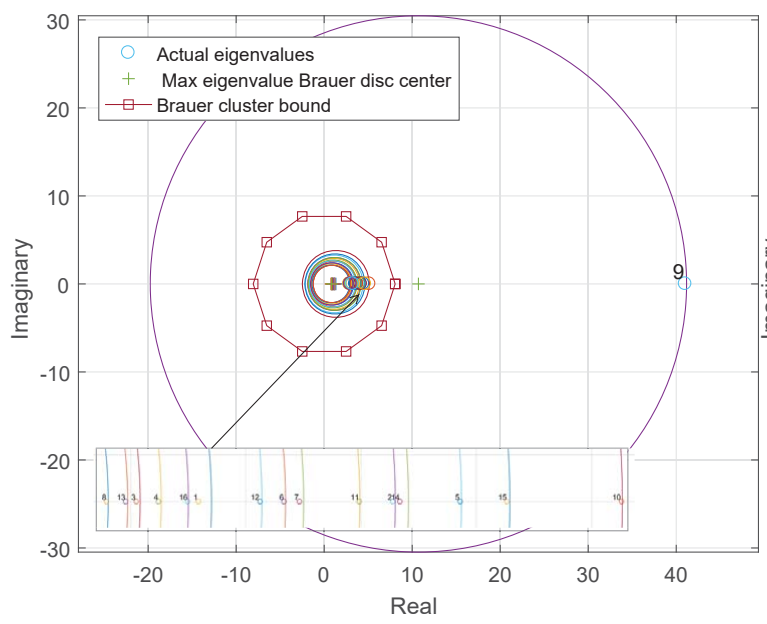

(a) ULA

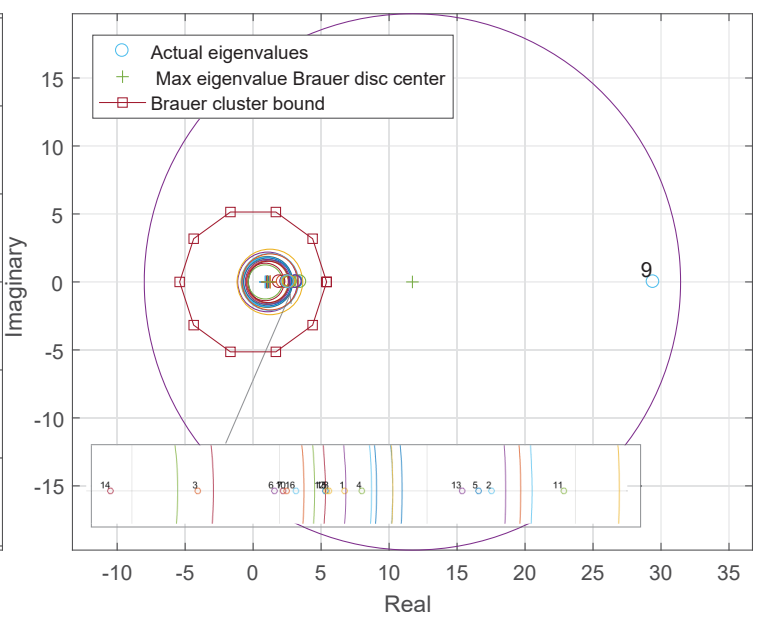

(b) RSLA

Figure 3.14: Brauer bound for clutter-plus-noise and potential target at the $9^{\text {th }}$ range bin for ULA and RSLA configurations for $5 \mathrm{~dB}$ SNR. 


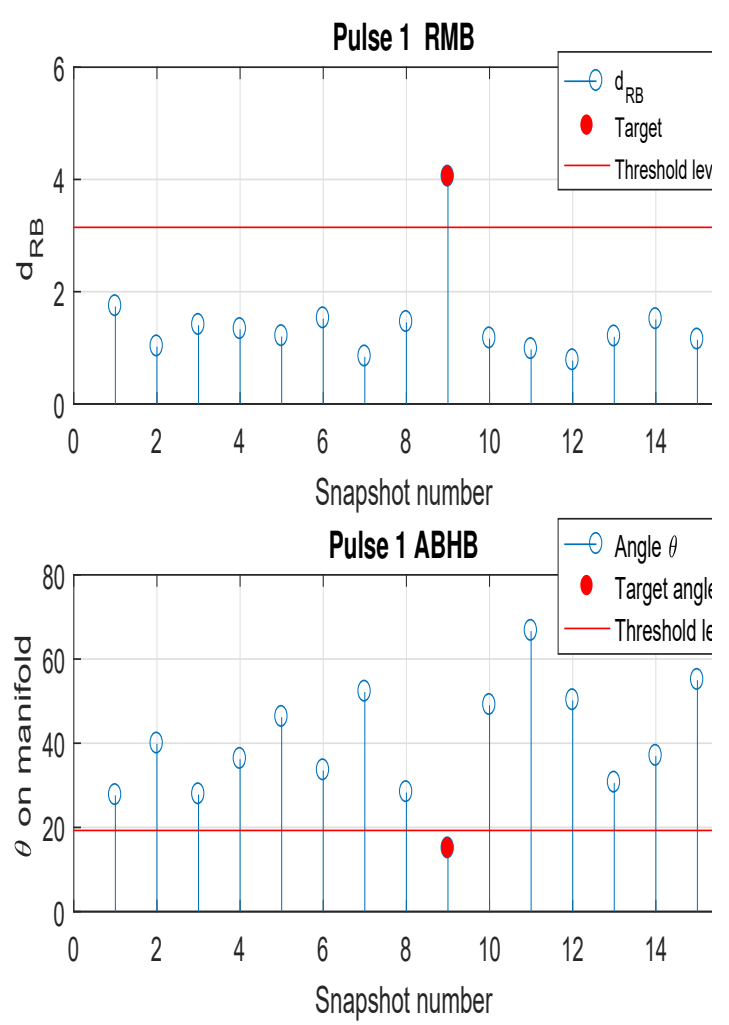

(a) ULA
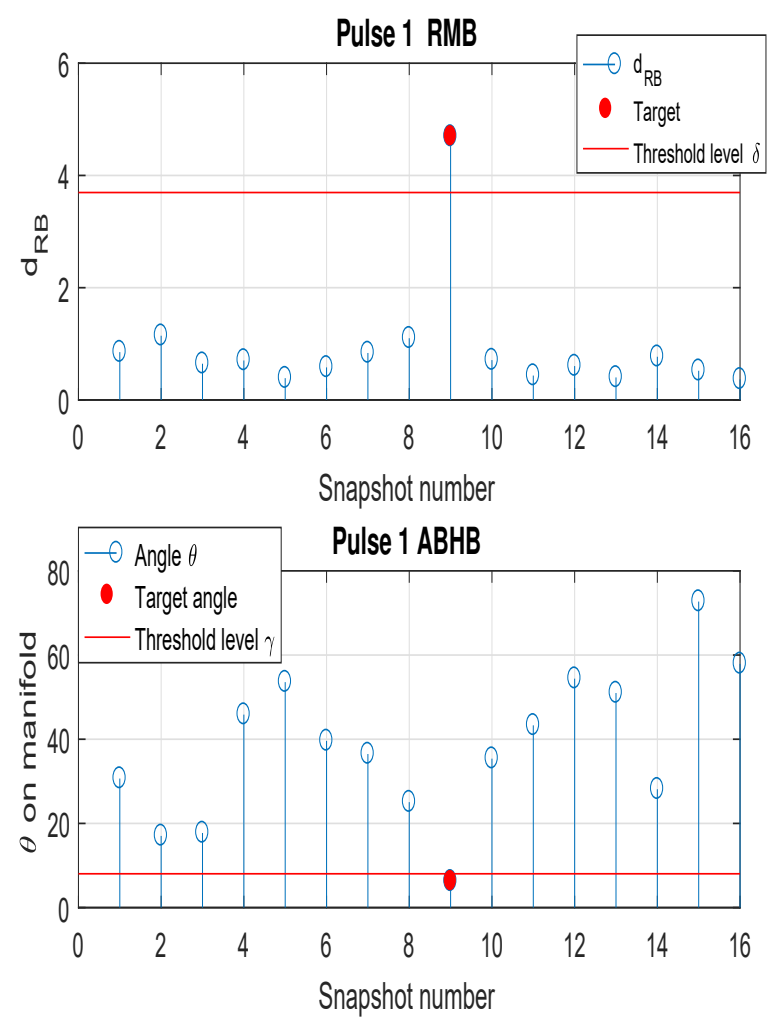

(b) RSLA

Figure 3.15: Detection performance of RBM and ABHB CFAR detectors for 16 snapshots pulse under $5 \mathrm{~dB}$ SNR. 

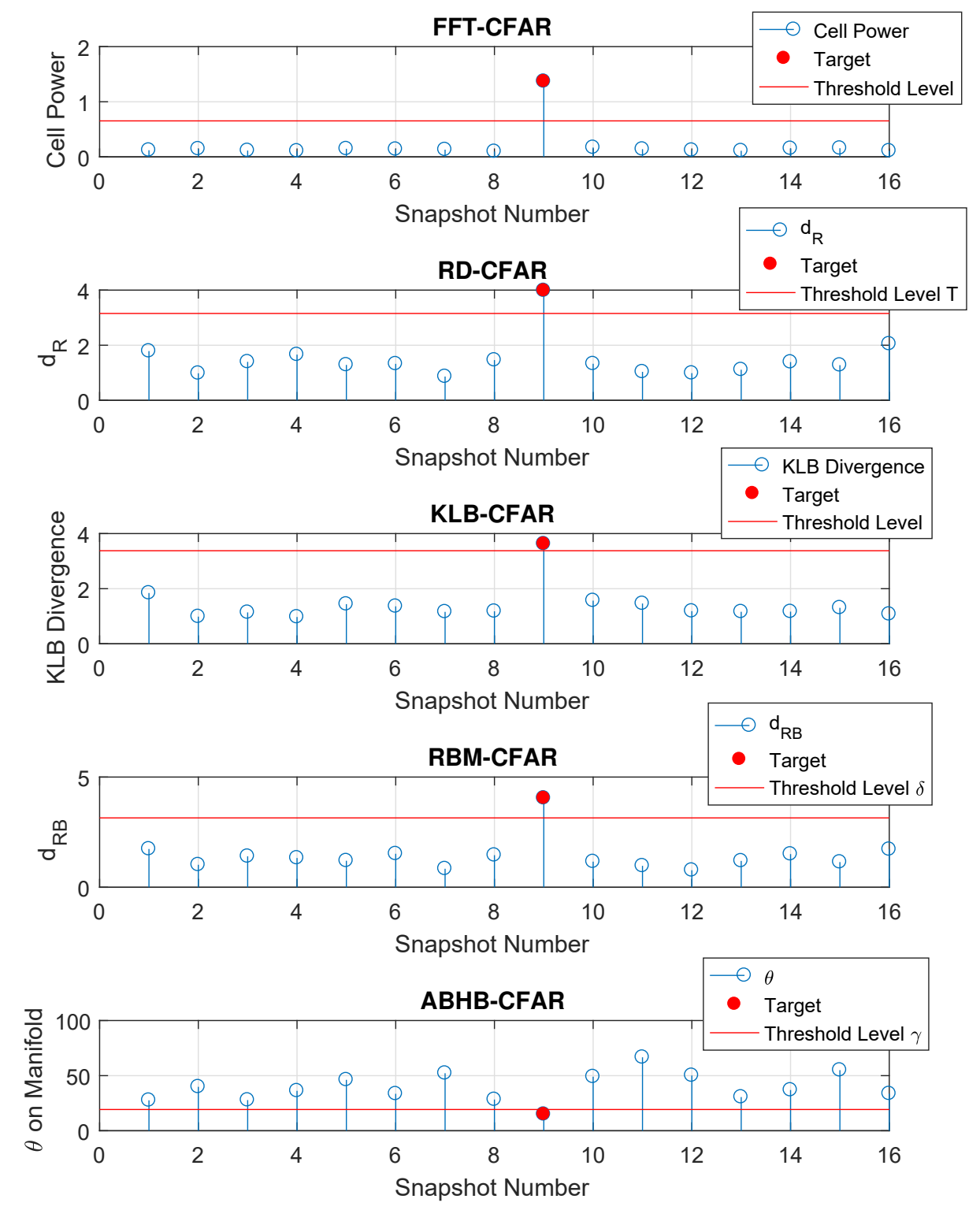

Figure 3.16: Detection performance of CA-CFAR, RD-CFAR, KLB-CFAR, RBMCFAR and ABHB-CFAR detectors for pulse 1 for ULA. 

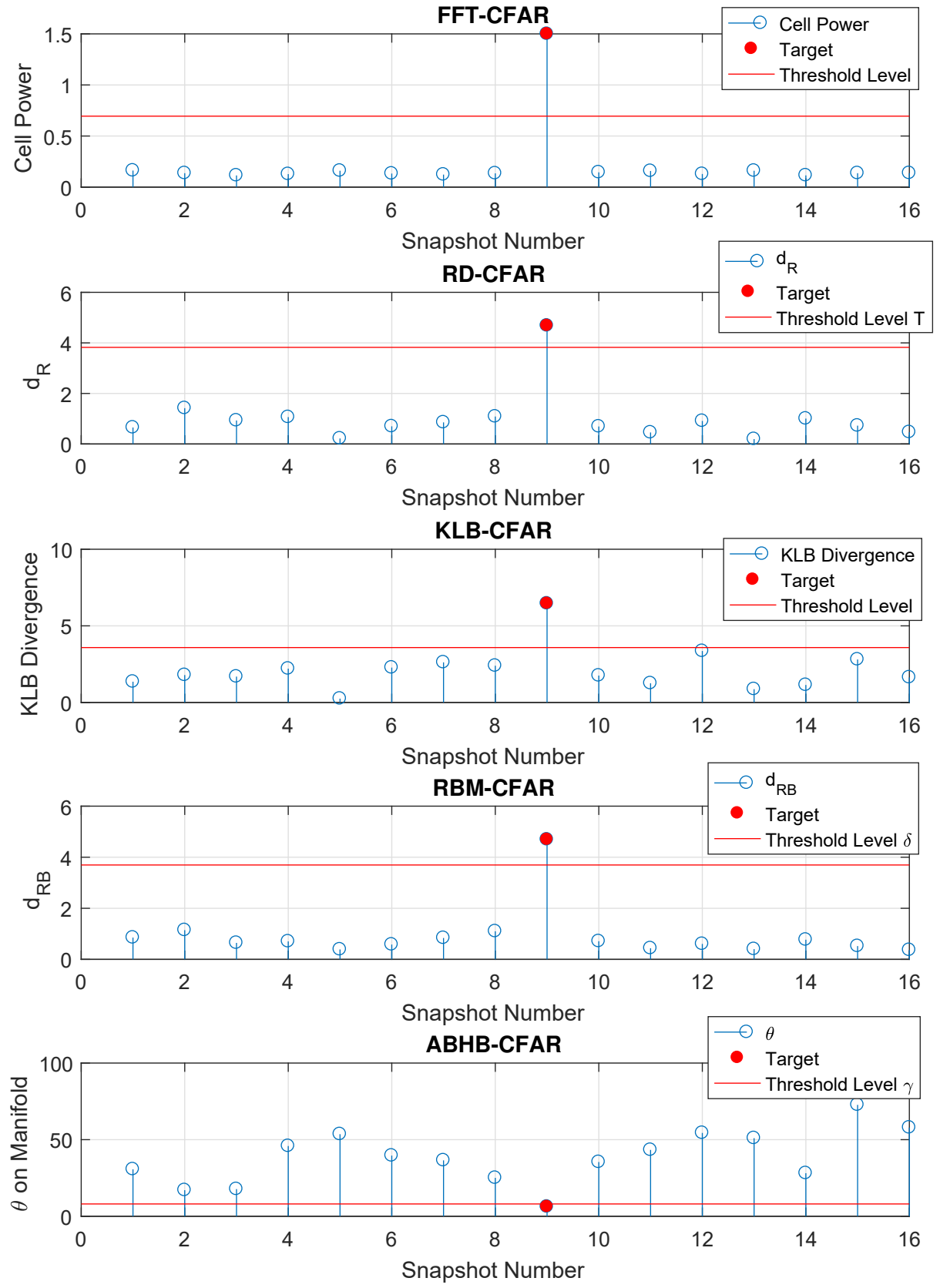

Figure 3.17: Detection performance of CA-CFAR, RD-CFAR, KLB-CFAR, RBMCFAR and ABHB-CFAR detectors for pulse 1 for RSLA. 


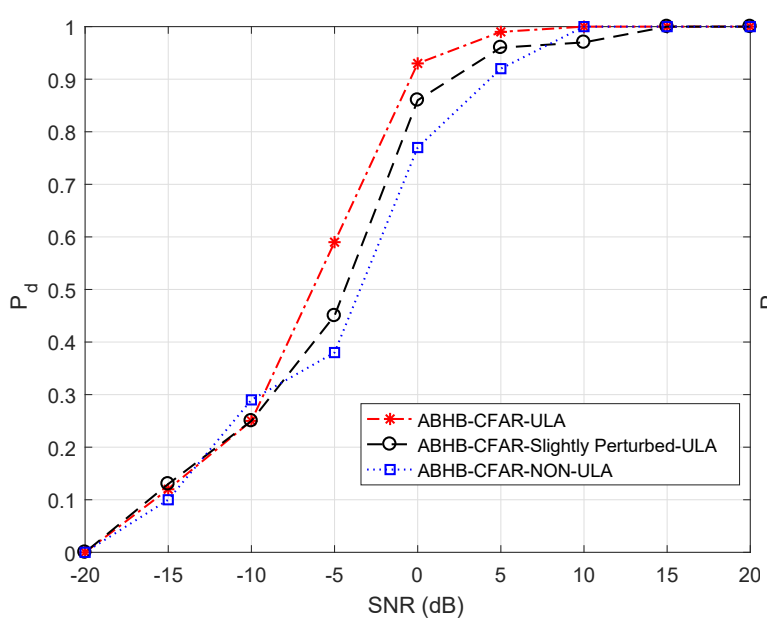

(a) ABHB-CFAR Detector

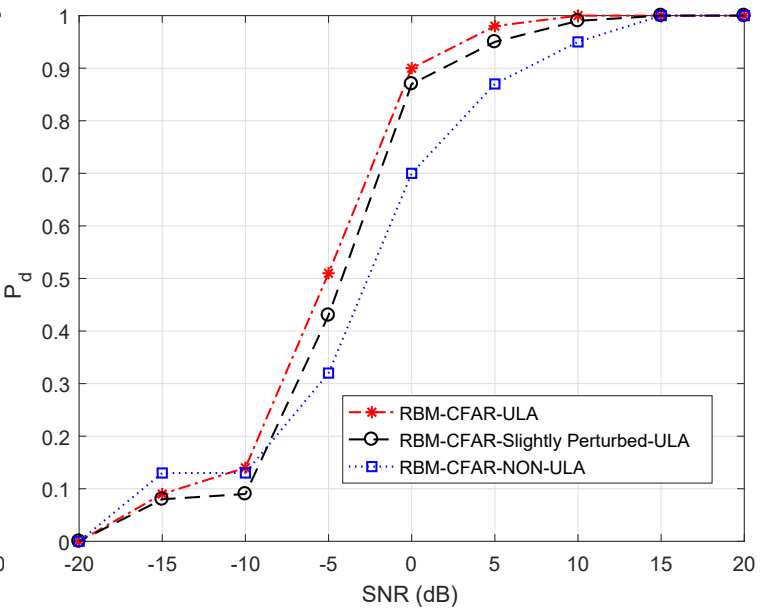

(b) RBM-CFAR Detector

Figure 3.18: Performance comparison of ABHB and RBM CFAR detectors for ULA, SPLA and RSLA for $P_{f}=10^{-4}$.

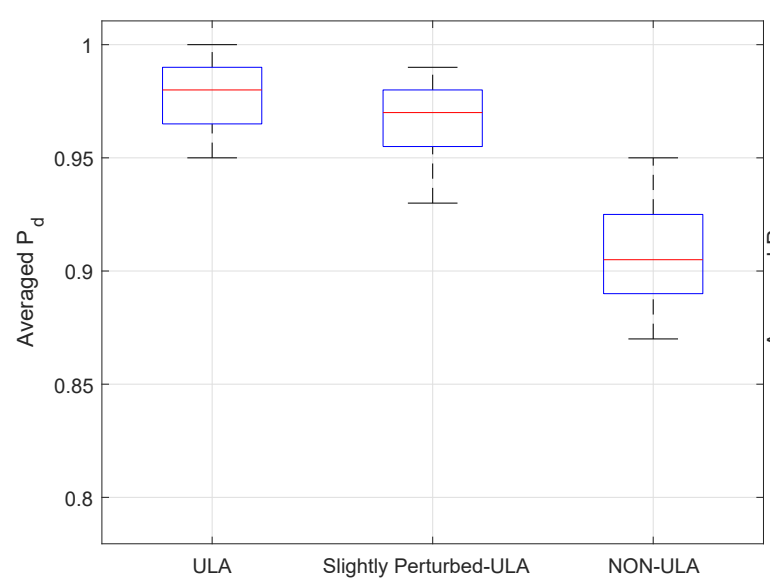

(a) ABHB-CFAR Detector

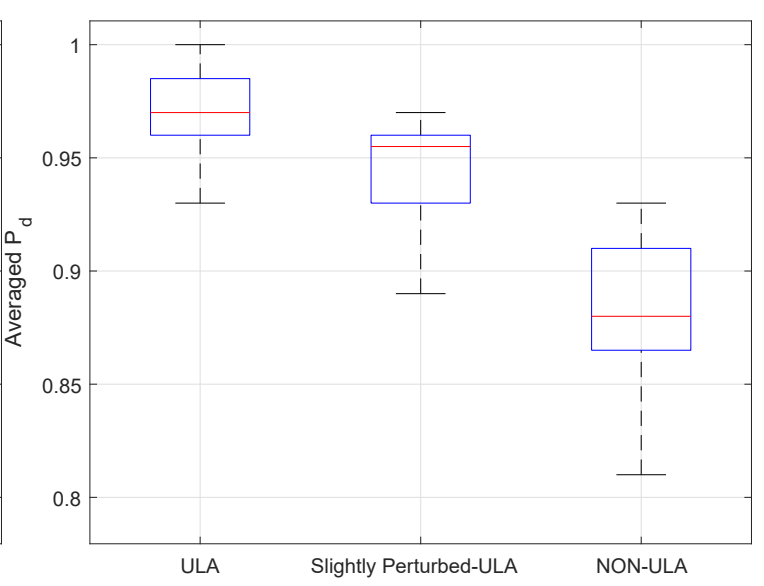

(b) RBM-CFAR Detector

Figure 3.19: Box plot for ABHB-CFAR and RBM-CFAR detectors for ULA, SPLA and RSLA configurations. 


\subsection{Real Data Analysis}

The performance of the RBM and ABHB CFAR detectors were tested on real data from a drone detection experiment. The data was collected using 1-D ULA Texas Instruments AWR1642 automotive radar with a moving Yuneec Typhoon H drone target. The AWR1642 radar is based on frequency-modulated continuous-wave (FMCW) with integrated phase locked loop and analog-to-digital converter. The experiment parameters and the radar specifications are given in Table 3.1.

\begin{tabular}{cc}
\hline \hline Feature & Specification \\
\hline \hline Centre freq. & $76-81 \mathrm{GHz}$ \\
Bandwidth & $4 \mathrm{GHz}$ \\
Pulse Repetition Frequency & $26.3 \mathrm{KHz}$ \\
Receive Channels & 4 Antennas \\
Tx Channels & 2 Antennas \\
Tx Power & $12.5 \mathrm{dBm}$ \\
Antenna spacing & $0.2 \mathrm{~cm}$ \\
Range Resolution & $3.75 \mathrm{~cm}$ \\
Sample freq. & $12.5 \mathrm{MHz}$ \\
Elevation Angle & 15 degrees \\
Azimuth Angle & 0 degrees \\
Max Range & 45 metres \\
Antenna Gain & $30 \mathrm{~dB}$ \\
\hline
\end{tabular}

Table 3.1: AWR1642 automotive radar specifications.

The performance of the proposed techniques is tested for 3 consecutive pulses of 16 range cells (snapshots) extracted from the data set. The extracted snapshots are converted to THPD covariance matrices using the RBA and a clutter-plus-noise bound using BD theorem was established. Figures. 3.20 to 3.22 illustrate the BD cluster bound for the maximum eigenvalues of the THPD covariance matrices. We notice that, there are two eigenvalues outside the BD cluster bound, the THPD covariance matrices associated with these two eigenvalues are considered potential targets. The 
detection threshold calculation is based on $P_{f}=10^{-3}$. In addition, Figs. 3.23 and 3.24 of the three consecutive pulses show some robustness in the performance of the RBM and ABHB techniques. For the RBM detector, both the Riemannian distance of the $9^{\text {th }}$ and $10^{\text {th }}$ snapshots' of the first pulse exceeded the detection threshold. Likewise, for ABHB, the $9^{\text {th }}$ and $10^{\text {th }}$ snapshots of the first pulse also have minimum angles. However, the AWR1642 data set contains only one target and the detection techniques are showing potentially two targets due to the range resolution of the AWR1642 being $3.75 \mathrm{~cm}$ and the target occupying two range bins (two snapshots). The $9^{\text {th }}$ snapshot contains most of the target's energy and the $10^{\text {th }}$ contains some of the target's energy. Furthermore, similar performance for pulses 2 and 3 are shown in Figs. 3.23 and 3.24 .

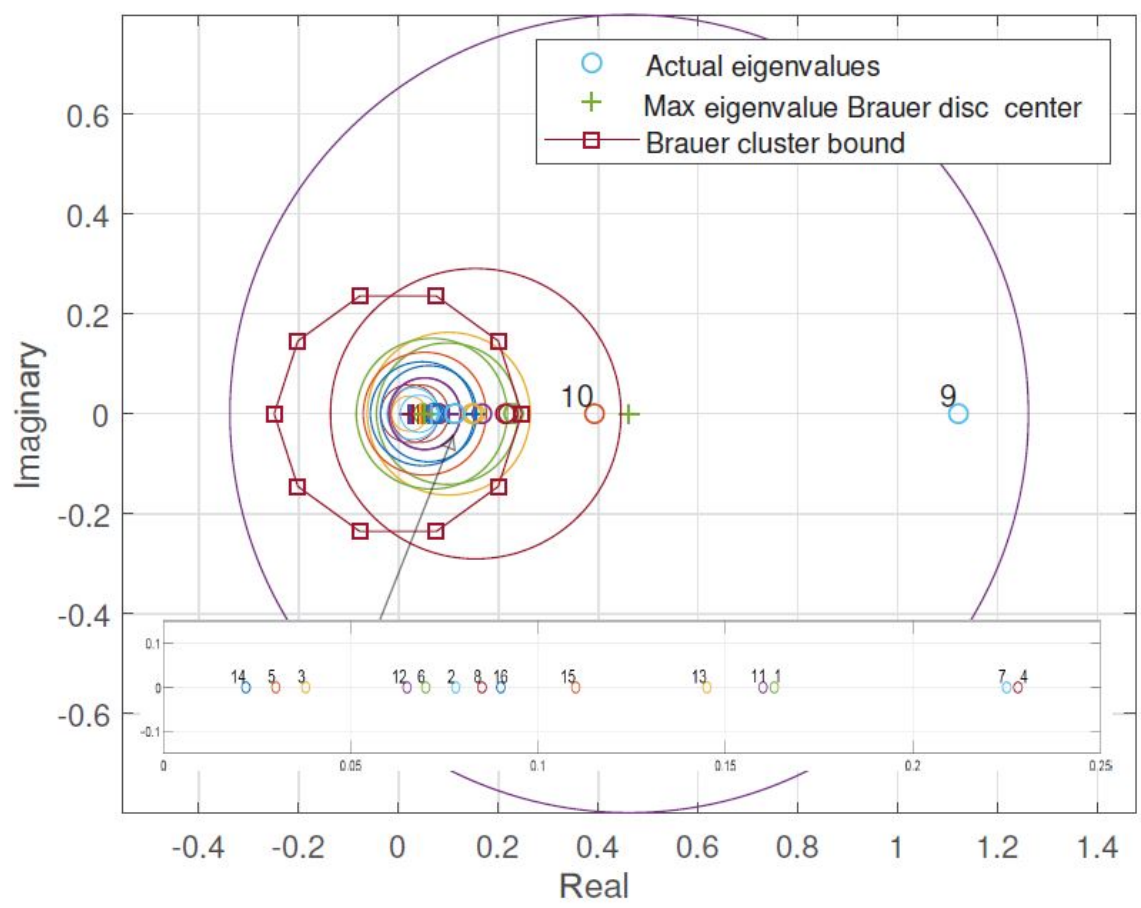

Figure 3.20: Brauer bound for clutter-plus-noise and potential targets at the $9^{\text {th }}$ and $10^{\text {th }}$ range bins for pulse 1 .

The performance of the RBM-CFAR and ABHB-CFAR techniques is compared with the CA-CFAR, the RD-CFAR and the KLB-CFAR as shown in Fig. 3.25. We 


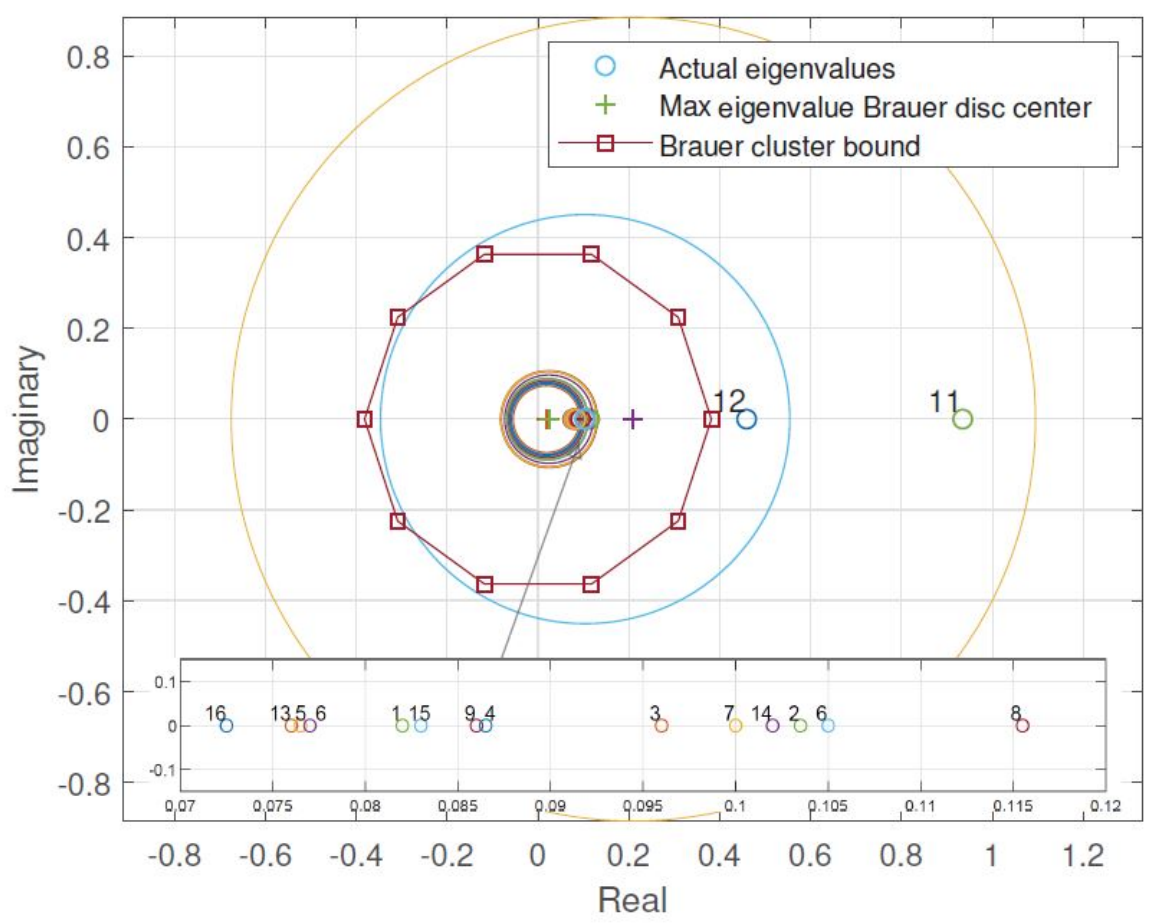

Figure 3.21: Brauer bound for clutter-plus-noise and potential targets at the $11^{\text {th }}$ and $12^{\text {th }}$ range bins for pulse 2 .

notice that all techniques detected the target at range bins 9 and 10 since the data collected has high SNR. By scaling the SNR by 0.2, the performance of the CA-CFAR and the RD-CFAR degraded in comparison to the KLB-CFAR, the RBM-CFAR and the ABHB-CFAR as shown in Fig. 3.26. In addition, the ABHB-CFAR and the RBMCFAR outperformed the KLB-CFAR and maintained the largest Riemannian distance and the smallest angle on the Riemannian manifold target point. Furthermore, the performance of the proposed CFAR detectors can be studied by varying the SNR of the real data and maintain a constant $P_{f}$. Figure 3.27 illustrates the detection performance at different $P_{f}$. We notice that at higher SNR, all CFAR detectors maintain a good detection performance. However, at lower SNR (less than $5 \mathrm{~dB}$ ), both the CA-CFAR and the RD-CFAR suffer degradation in performance in comparison with the other CFARs due to the limited number of range cells. Also, at lower $P_{f}$ the RBM-CFAR and the ABHB-CFAR maintained a superior performance while the KLB-CFAR, the CA-CFAR and the RD-CFAR failed to retain detection performance. 


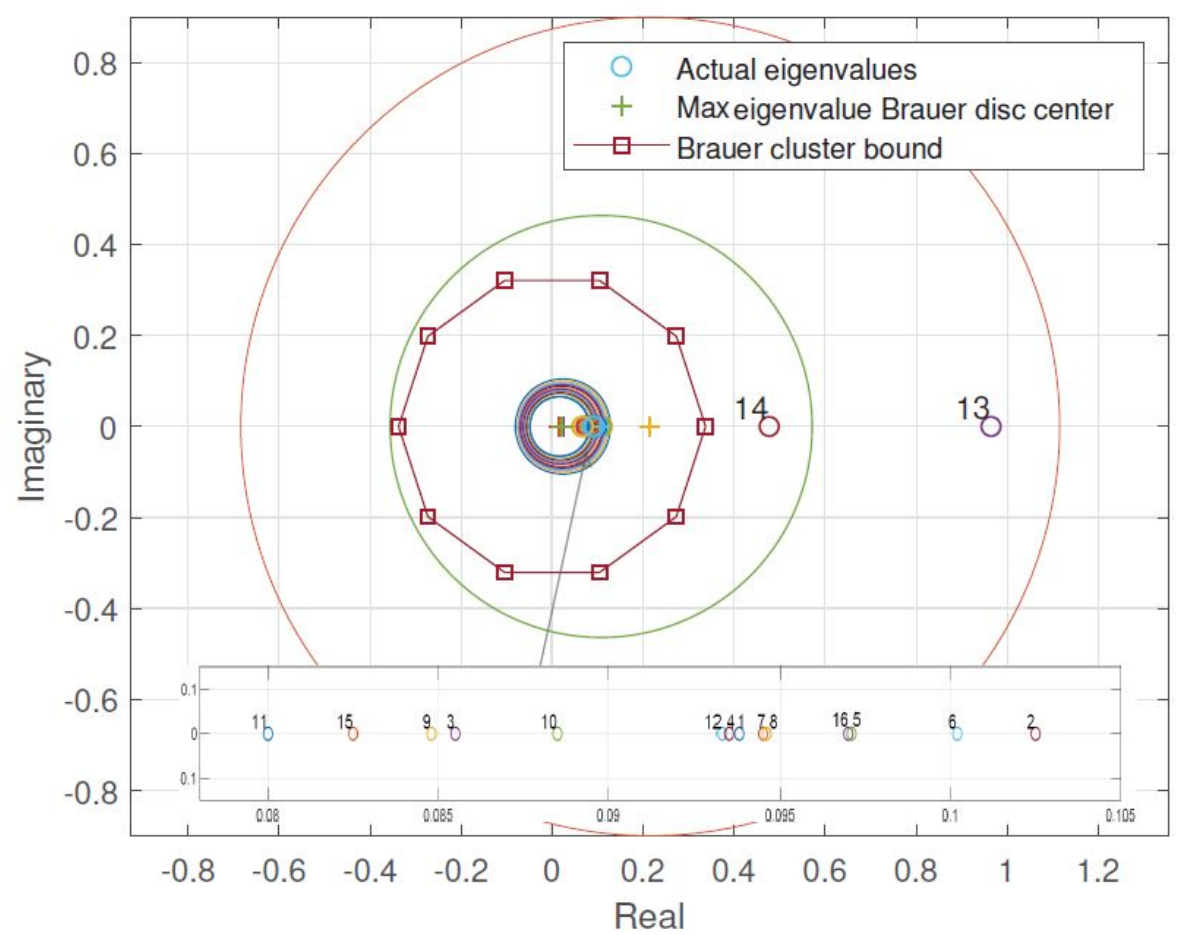

Figure 3.22: Brauer bound for clutter-plus-noise and potential targets at the $13^{\text {th }}$ and $14^{\text {th }}$ range bins for pulse 3 . 

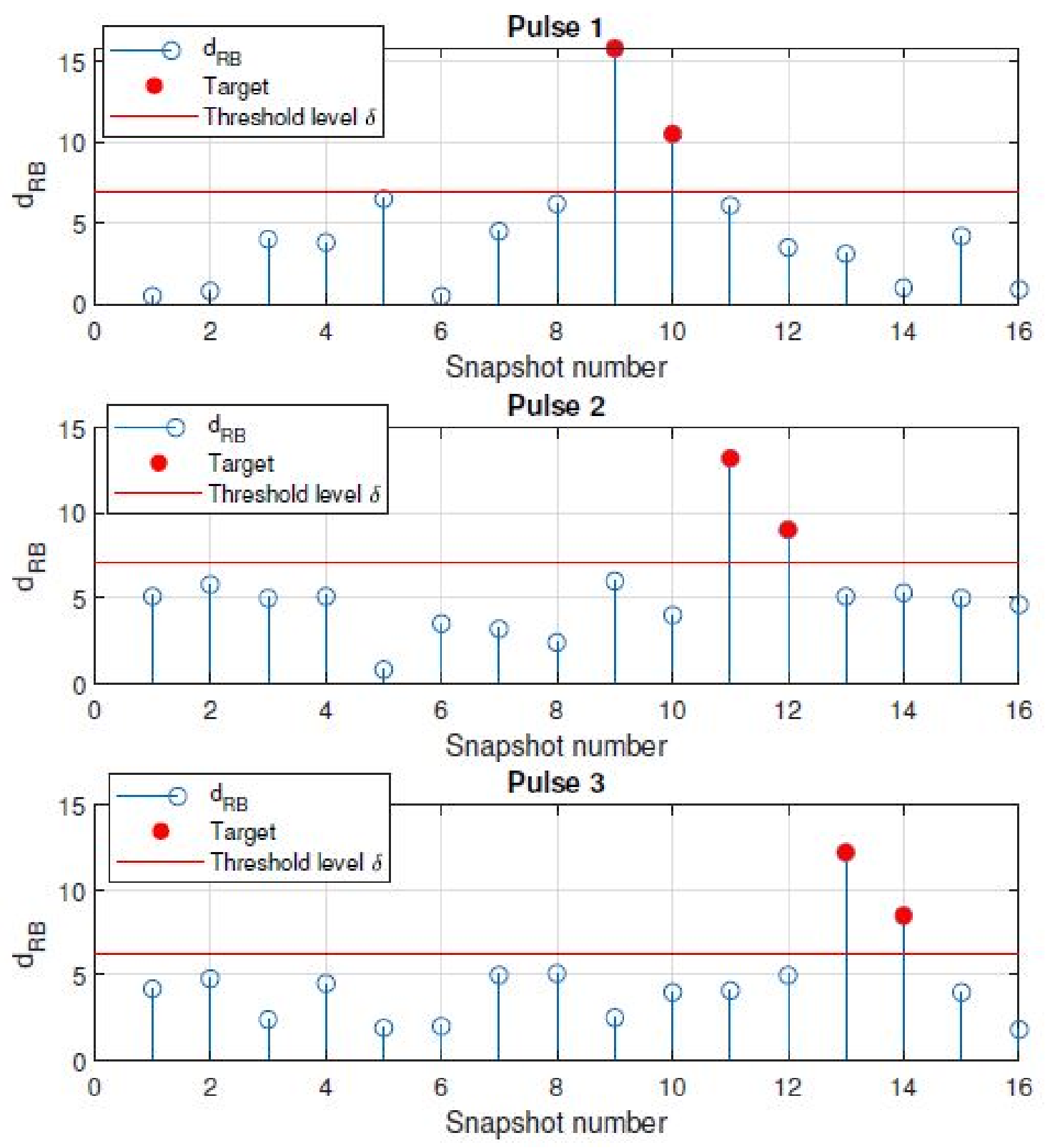

Figure 3.23: Detection performance of the RBM-CFAR detector for three consecutive pulses. 

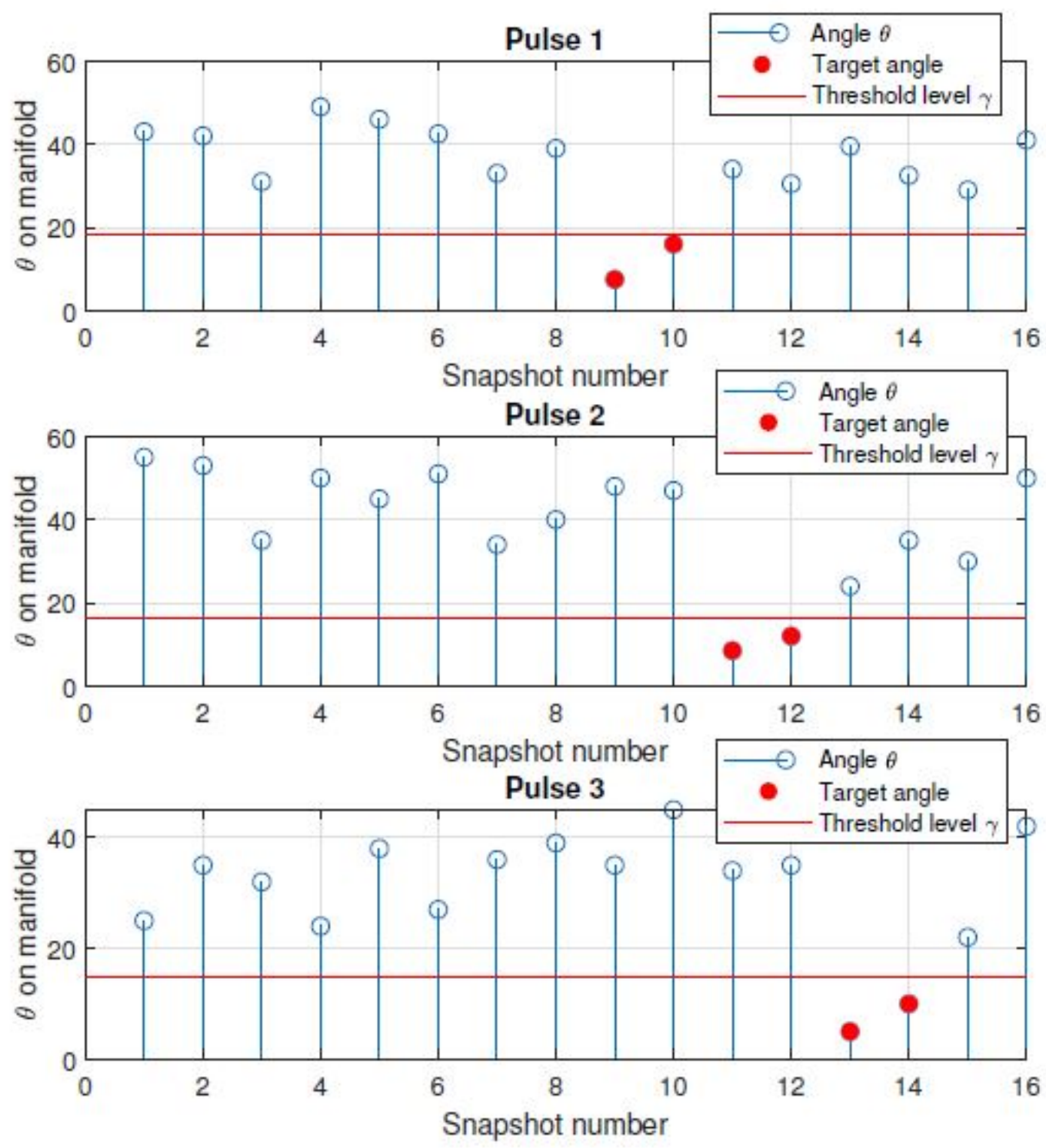

Figure 3.24: Detection performance of the ABHB-CFAR detector for three consecutive pulses. 

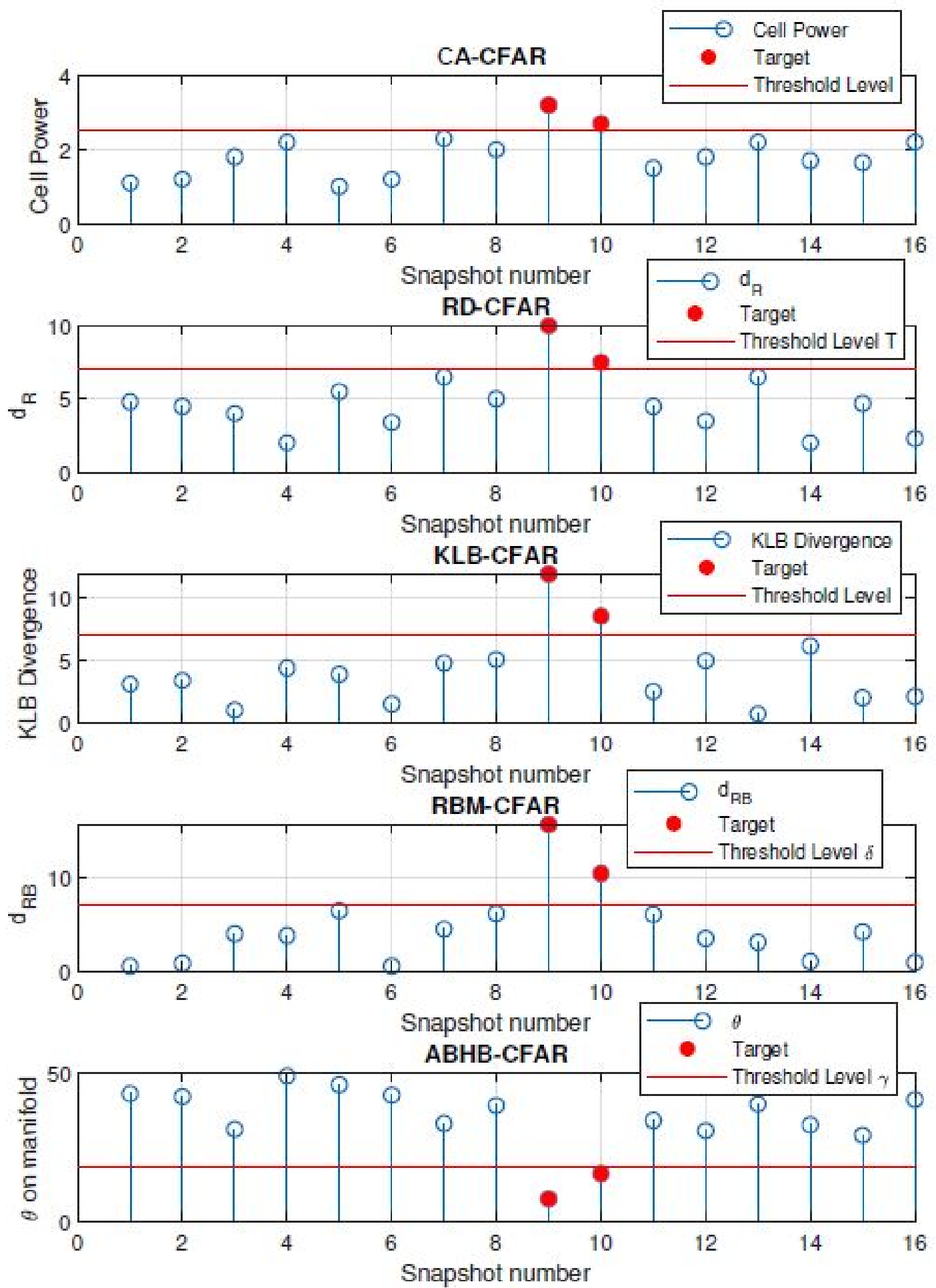

Figure 3.25: Detection performance of the CA-CFAR, the RD-CFAR, the KLBCFAR, the RBM-CFAR and the ABHB-CFAR detectors for pulse 1 . 

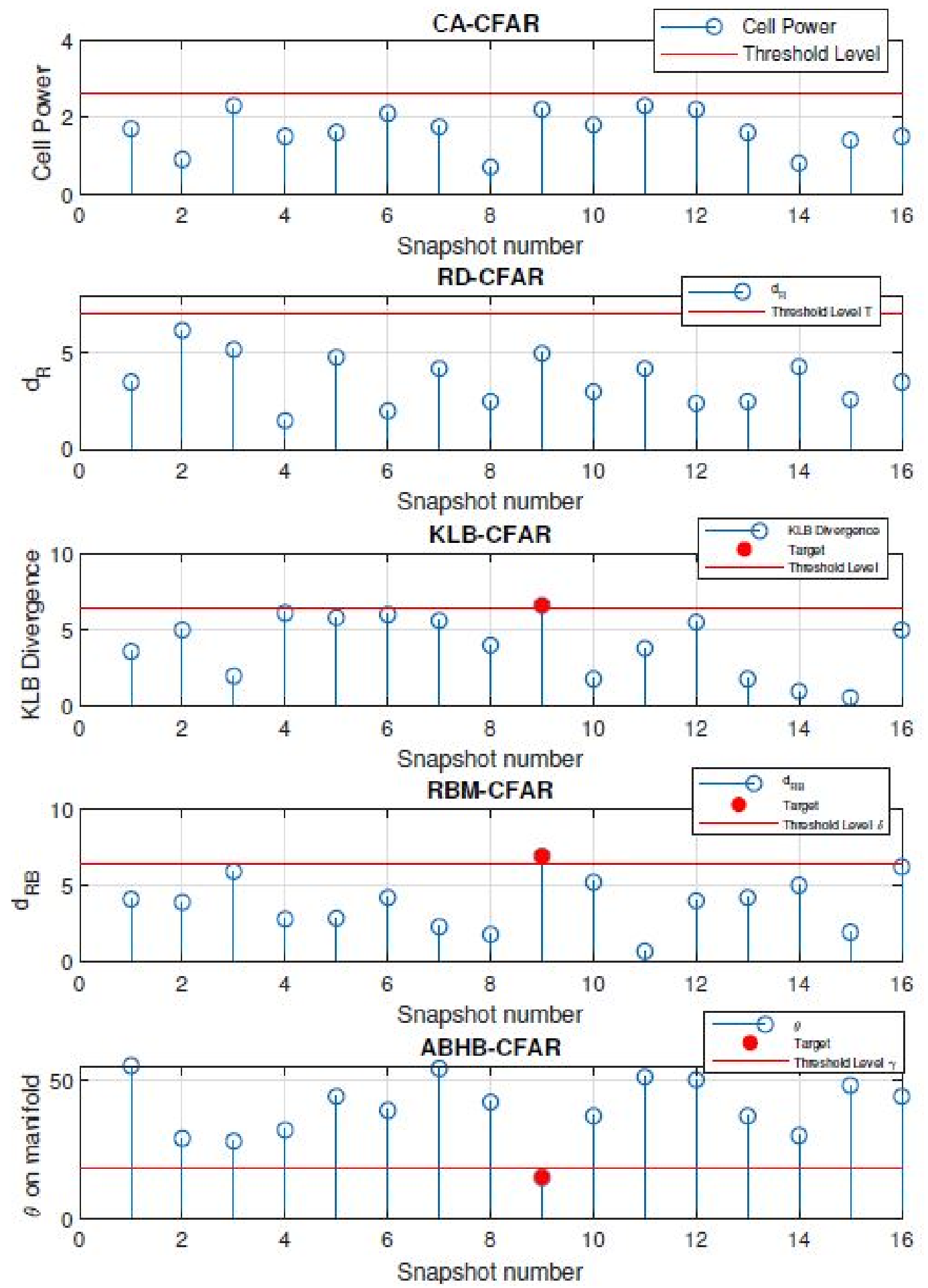

Figure 3.26: Detection performance of the CA-CFAR, the RD-CFAR, the KLBCFAR, the RBM-CFAR and the ABHB-CFAR detectors under scaled SNR for pulse 1. 


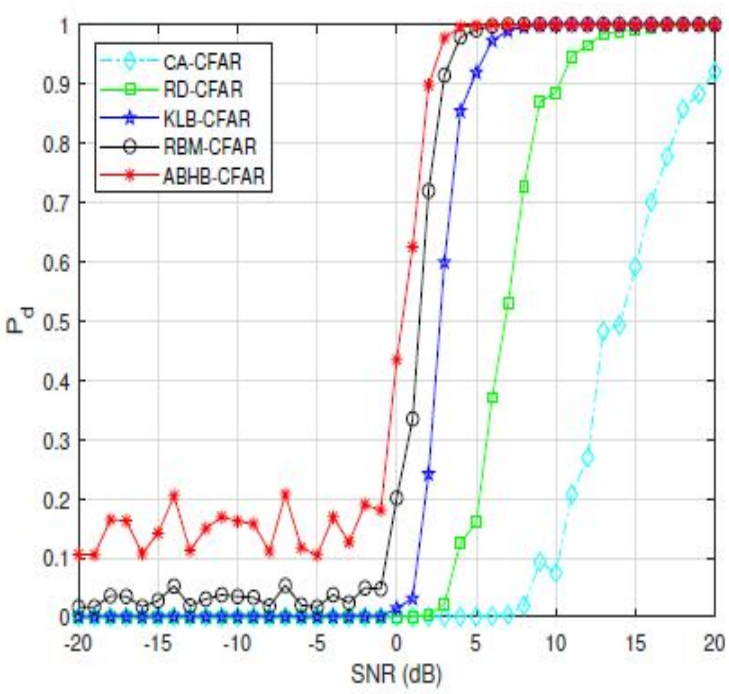

(a) $P_{f}=10^{-4}$

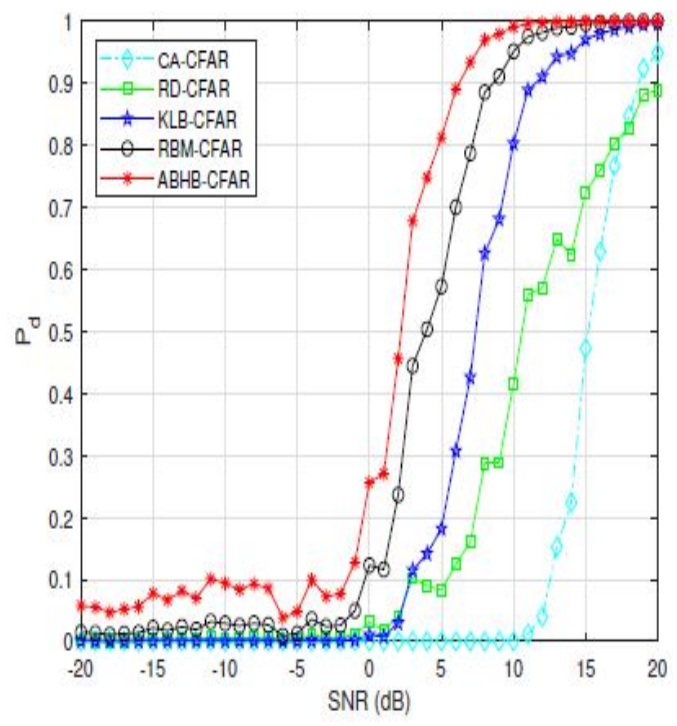

(c) $P_{f}=10^{-6}$

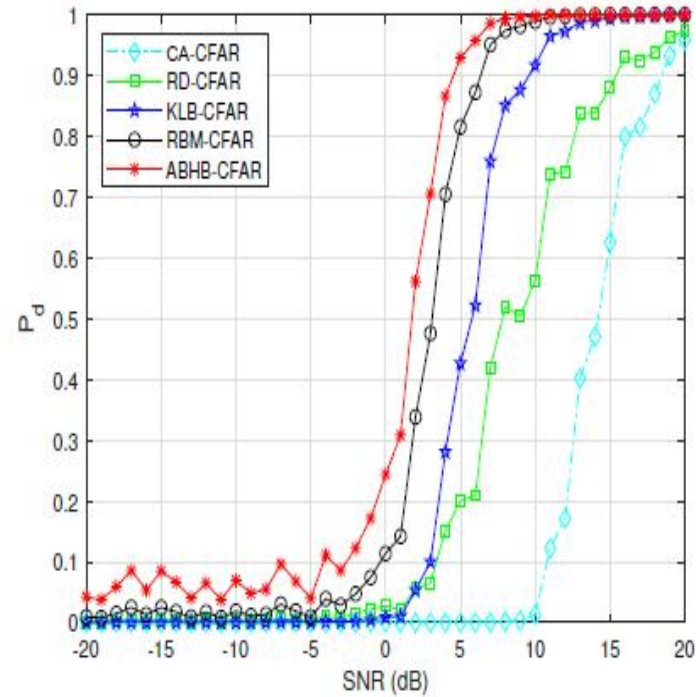

(b) $P_{f}=10^{-5}$

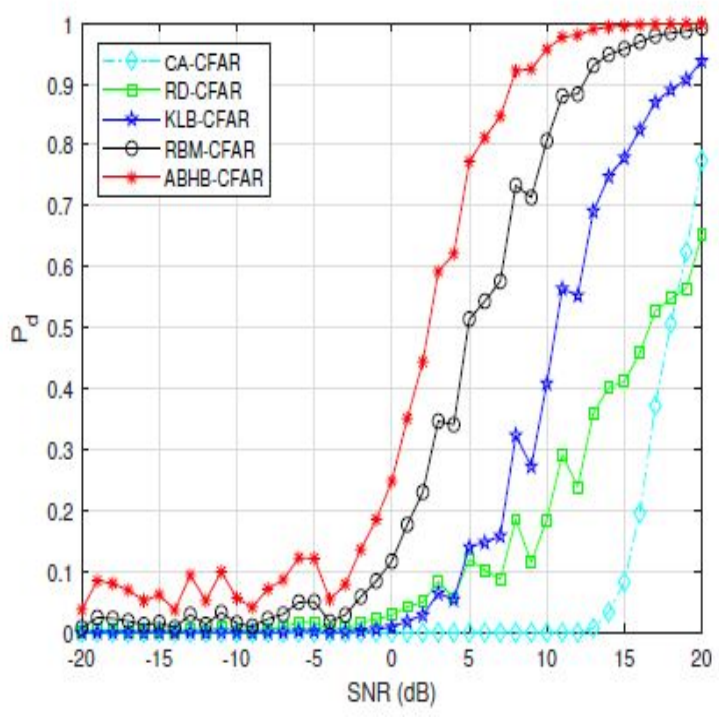

(d) $P_{f}=10^{-7}$

Figure 3.27: Performance comparison of the CA-CFAR, the RD-CFAR, the KLBCFAR, the RBM-CFAR and the ABHB-CFAR detectors for different $P_{f}$. 


\subsection{Conclusion}

The drone detection problem is viewed from a Riemannian geometry perspective. Two novel CFAR detection techniques,namely the RBM- and ABHB-CFAR techniques were formulated to improve the probability of detection under small sample size with low SCR. The RBM-CFAR and the ABHB-CFAR showed an improvement in probability of detection in comparison with the CA-CFAR, the RD-CFAR and the KLB-CFAR. In addition, the BD theorem provided robustness to the calculation of the Riemannian mean and median by excluding outliers from the Riemannian mean and median computations. Varying the spacing between antenna elements slightly or randomly affects the propagation delay between antennas, which affects the eigenvalues of the estimated covariance matrices. This resulted in 1 to 2.5 loss in the detection probability in comparison with ULA configuration detection. Even real data analysis proved that the proposed CFAR detectors can achieve better performance for low SCR, low sample size data and high probability of false alarm.

The Riemannian based approach exploited the information geometry present in every range bin and the BD theorem to distinguish between clutter-plus-noise and potential targets covariance matrices. Hence, increasing the number of clutter-plusnoise covariance matrices for estimating the clutter or noise power for limited number of range bins, led to better performance for RBM-CFAR and ABHB-CFAR when compared to the state of the art techniques. In addition, the ABHB-CFAR showed a better performance in comparison with the RBM-CFAR under low SCR. This is due to the fact that the RBM-CFAR detectors is based on Riemannian distance, which may encounter numerical difficulties in high dimensions, hence the angle on the Riemannian manifold provides better metric for detection. 


\section{Chapter 4}

\section{Direction of Arrival Estimation in the Riemannian Space}

Direction-of-arrival (DOA) estimation refers to the process of retrieving the direction information of several electromagnetic waves/sources from the outputs of a number of receiving antennas that form a sensor array. DOA estimation is a major problem in array signal processing and has wide applications in radar, sonar and wireless communications.

This chapter provides a brief survey of the various techniques for the estimation of DOA of radar signals using a uniform linear antenna array (ULA). The array-based DOA estimation techniques discussed in this chapter are divided into four different types: conventional techniques, subspace-based techniques, compressive sensing techniques and Riemannian geometry-based technique. In addition, this chapter proposes a new Riemannian geometry (RG) DOA estimation technique. This technique exploits the geometry present in the space of probability distributions parametrized by their covariance matrix where every covariance matrix represents a point on the Riemannian manifold. The RGDOA estimation problem is formulated as a linear search for minimum Riemannian distance between steering vector Hermitian positive define (HPD) matrices and the Riemannian mean.

This chapter also discusses the performance of the RGDOA estimation technique for ULA configuration and provides a performance comparison with the RSLA configuration. 


\subsection{Uniform Linear Antenna Array and System Signal Model}

The array antenna is a major player in various applications ranging from military use to commercial use, e.g., passive, active and airborne radar systems, mobile communication, and radio astronomy [85]. One of the main functions of an antenna array is to perform spatial sampling of the incoming signal sources for estimating the DOA of the signal sources, and the number of transmitted and interference signal sources [86]. DOA estimation is one of the major applications of the antenna array. The number of sources that can be resolved depends on the degree of freedom of the antenna array, which is closely related to array configuration. Also, DOA estimation accuracy with an antenna array depends on the type of the antenna array and its size.

The simplest and most frequently applied array structure is the uniform linear antenna (ULA). A ULA structure is very useful and easy to apply to any RF devices to be used for DOA estimation [87]. The number of elements in a ULA can be arbitrary and the spacing between any two consecutive antenna elements is constant $d$ as shown in Fig. 4.1. In general, the spacing $d$ is less than the half wavelength corresponding to the highest frequency of received signal sources in order to avoid sidelobes and grating lobes in the spatial spectrum.

The DOA estimation accuracy has been mostly confined to the case of ULA configurations [86]. Non uniform array configurations, such as nested arrays, can also be used to estimate the DOA, however this will lead to non-Toeplitz structure of the sample covariance matrix, which may affect the DOA estimation performance.

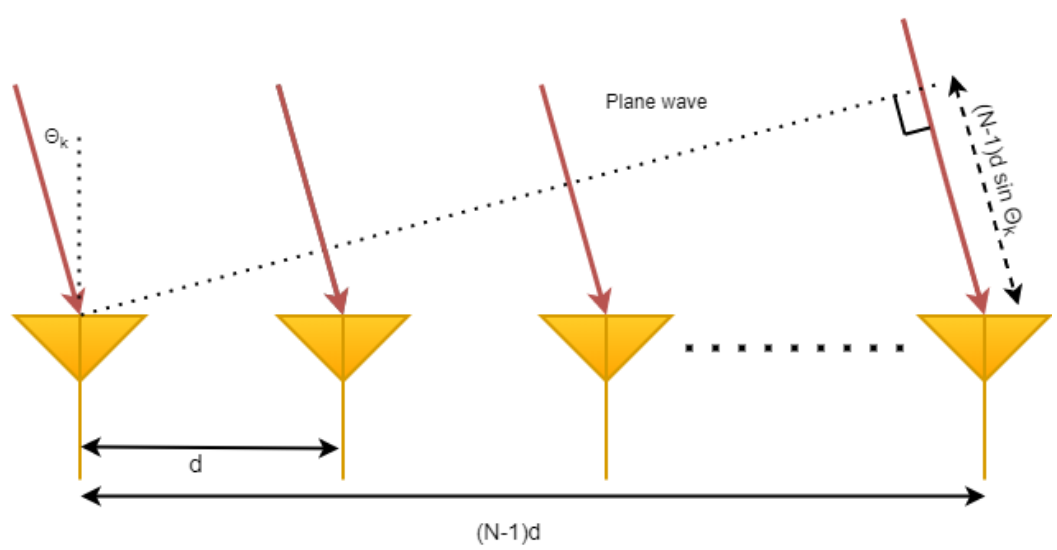

Figure 4.1: ULA structure with signal impinging from a particular direction. 
The narrowband system model is based on ULA with $N$ antennas and antenna spacing $d$ is equal to $\lambda / 2$, where $\lambda$ is the wavelength of the carrier frequency. Consider estimating the DOA of $L$ incoming signal sources, where $L \leq N$ [88]. The received signal at the $n t h$ antenna can be expressed as

$$
\mathbf{z}(t)=\mathbf{s}(t) \mathbf{A}(\theta)+\mathbf{n}(t)
$$

where $\mathbf{s}(t)=\left[s_{0}(t), s_{1}(t), \ldots, s_{L-1}(t)\right]^{T}$ denotes the signal source vector, $\mathbf{n}(t)$ denotes the additive white complex Gaussian noise and $\mathbf{A}(\theta)$ is the array manifold matrix, which can be expressed as

$$
\mathbf{A}(\theta)=\left[\mathbf{a}\left(\theta_{0}\right), \mathbf{a}\left(\theta_{1}\right), \ldots, \mathbf{a}\left(\theta_{L-1}\right)\right]^{T},
$$

where

$$
\mathbf{a}\left(\theta_{l}\right)=\left[1, \exp \left(-j \frac{2 \pi}{\lambda} d \sin \theta_{l}\right), \ldots, \exp \left(-j \frac{2 \pi}{\lambda} d(N-1) \sin \theta_{l}\right)\right] .
$$

The correlation matrix of $\mathbf{z}(t)$ can be expressed as

$$
\begin{aligned}
\mathbf{R}_{z} & =E\left[\mathbf{z z}^{H}\right] \\
& =\mathbf{A}(\theta) \mathbf{R}_{s} \mathbf{A}^{H}(\theta)+\sigma_{n}^{2} \mathbf{I},
\end{aligned}
$$

where $\mathbf{R}_{s}=E\left[\mathbf{s}(t) \mathbf{s}(t)^{H}\right], \sigma_{n}^{2}$ is the noise power and $\mathbf{I}$ is an $N \times N$ unit matrix.

\subsection{Overview of DOA Estimation Techniques}

There are many techniques used for estimating the DOA, such as delay and sum (DAS), minimum variance distortionless response (MVDR), subspace techniques like multiple signal classification and estimation of signal parameters via rotational invariance techniques (ESPRIT), compressive sensing, such as $\ell_{2,1}$ and weighted spatial filter (WSF) $\ell_{2,1}$, and the Riemannian geometry-based technique.

DAS and MVDR are two conventional methods used for DOA estimation. Unlike other DOA estimation methods, conventional DOA estimation methods does not exploit the nature of the received signal vector or the statistical model of the signals and noise. These techniques steer the beams electronically in all possible directions 
looking for peaks in the output power [89]. The DAS method, also known as the Fourier method, is based on combining the received signals linearly using complex weights (classical beamformer). The output power $P_{b}$ of the DAS method can be written in terms of the angle of arrival $\theta$ as

$$
P_{b}=\mathbf{w}^{H} \mathbf{R}_{z z} \mathbf{w}=\mathbf{a}^{H}(\theta) \mathbf{R}_{z z} \mathbf{a}(\theta)
$$

where $\mathbf{w}$ is the complex weights vector. The DAS method is easy to implement and does not require a lot of computations. On the other hand, the width of the beam and the height of the sidelobes from multiple directions limit its performance and reduce the resolution. In addition, the DAS technique requires a large number of antenna elements to achieve high resolution. More details are provided in Appendix A.1.1

Capon's method [90], also known as MVDR, can overcome these issues presented in the DAS technique and provide better resolution. MVDR uses some of the degrees of freedom to form a beam in the desired look direction while simultaneously using the remaining degrees of freedom to form nulls in the direction of interfering signals. It also minimizes the contribution of the undesired interferences and jammers by minimizing the output power while maintaining a unity gain along the look direction [91]. This techniques can be expressed as the following optimization problem [92]

$$
\begin{array}{ll}
\underset{\mathbf{w}}{\min } & \mathbf{w}^{H} \mathbf{R}_{z z} \mathbf{w} \\
\text { s.t. } & \mathbf{w}^{H} \mathbf{a}\left(\theta_{0}\right) .
\end{array}
$$

The solution for this optimization problem can be obtained by minimizing the variance (average power) of the output signal. The weight vector $\mathbf{w}$ can be shown to be [93]

$$
\mathbf{w}=\frac{\mathbf{R}_{z z}^{-1} \mathbf{a}(\theta)}{\mathbf{a}^{H}(\theta) \mathbf{R}_{z z}^{-1} \mathbf{a}(\theta)} .
$$

MVDR provides a better resolution when compared to the DAS method. However, the performance of MVDR degrades if other received signals are correlated with the signal of interest. In addition, MVDR suffers from self nulling in the case of DOA mismatch [94]. See Appendix A.1.2 for more details.

The conventional techniques have some limitations in resolution and they do not 
exploit the structure of the input signal model of the measurements. Subspace techniques, such as MUSIC and ESPRIT, exploit the structure of a data model [95]. The geometric concepts upon which MUSIC is founded form the basis for a much broader class of subspace-based algorithms [96]. In addition to the MUSIC algorithm, another subspace-based algorithm called estimation of signal parameters via rotational invariance technique (ESPRIT) was proposed by Roy and Kailath et al. [97]. The MUSIC algorithm is based on exploiting the eigen-structure of the input covariance matrix. The basic idea of the MUSIC algorithm is to conduct the characteristic decomposition of the covariance matrix of any array output data, resulting in a signal subspace orthogonal to a noise subspace corresponding to the signal components. These two orthogonal subspaces are used to form a spatial spectrum function where its peaks are the DOA estimates. The MUSIC algorithm has a high resolution, accuracy and stability under certain conditions. The direction of arrival of the multiple incident signals can be estimated by locating the peaks of a MUSIC spatial spectrum $P_{M U S I C}(\theta)$ given by [98]

$$
P_{M U S I C}(\theta)=\frac{1}{\mathbf{a}^{H}(\theta) \mathbf{V}_{n} \mathbf{V}_{n}^{H} \mathbf{a}(\theta)}
$$

where $\mathbf{V}_{n}$ is the matrix of non-principal eigenvectors associated with noise. In general, MUSIC has many advantages, such as the ability to simultaneously measure multiple signals, to provide spectrum with high precision and to produce high resolution for antenna beam signals [99]. For the derivation of MUSIC algorithm see Appendix A.2.1.

ESPRIT is another subspace based algorithm, which does not involve an exhaustive search through all possible steering vectors to estimate DOA and dramatically reduces the computational and storage requirements in comparison with the MUSIC algorithm. In addition, ESPRIT does not require that the array manifold vectors be precisely known, and hence the calibration requirements are not stringent. The goal of the ESPRIT technique is to exploit the rotational invariance in the signal subspace which is created by two equal size identical sub-arrays with a translational invariance structure, that is, the array should possess a displacement (translational) invariance, and the sensors should occur in matched pairs with identical displacement [97]. The direction of arrival of the $k^{t h}$ source signal can be estimated using the phased delay 
between the sub-array sensors and can be expressed as

$$
\theta_{k}=\cos ^{-1}\left[c \frac{\arg \left(\Phi_{k}\right)}{\omega_{0} \Delta}\right]
$$

where $\Phi_{k}$ is the eigenvalue of the phased delay matrix of the sub-array sensors, $\Delta$ is the translational vector, $c$ is the speed of propagation and $\omega_{0}$ is the carrier angular frequency. More details on ESPRIT algorithm see Appendix A.2.2.

Compressive sensing (CS) is defined as a method for acquisition of sparse signals by sampling well below the Nyquist rate and reconstruction of sparse signals from compressed measurements [100]. The DOA estimation is liked to the CS framework through the underlying spatial sparsity reconstruction problem. DOA estimation from the perspective of sparse signal reconstruction brings benefits such as smaller number of required data samples and ability to deal with highly correlated and coherent sources [101]. A lot of applications related to the direction finding problem under the CS framework with limited number of snapshots have been investigated $[102,103]$. The $\ell_{2,1}$-norm penalty can be used to estimate the DOA with a few snapshots. The matrix form of the received signals can be written as

$$
\mathbf{Z}=\mathbf{A X}+\mathbf{N}
$$

where $\mathbf{A}$ is the array manifold, $\mathbf{X}$ is the joint source signals matrix and $\mathbf{N}$ is the noise matrix. The DOA estimation problem can be formulated as an $\ell_{2,1}$-minimization problem, which can be expressed as

$$
\begin{array}{ll}
\min & \|\mathbf{X}\|_{2,1} \\
\text { subject to } & \|\mathbf{Z}-\mathbf{A X}\|_{F} \leqslant \epsilon,
\end{array}
$$

where $\epsilon$ is a small constant to limit the residual error and $\|\mathbf{X}\|_{2,1}=\sum_{p} \sqrt{\sum_{t} x^{2}\left(\theta_{p}, t\right)}$. Once the optimization problem converges, the peaks of the $\ell_{2}$-norm of each row in $\mathbf{X}$ are the estimated DOAs [104].

The $\ell_{2,1}$-minimization problem in (4.11) assumes time invariance of multiple snapshots. However, its performance can easily degrade in a low SNR environment, with fewer sensor elements and very small sample size. According to [103], exploiting a weighted spatial filter (WSF) matrix for sparse constraint by some prior information can improve the algorithm performance. The WSF- $\ell_{2,1}$ constructs the spatial filter 
weighting matrix $\mathbf{Q}$ by dividing the received signals into two parts: the reference signals which are received by the first sensor and the input signals which are received by other sensors [104]. Thus, the WSF- $\ell_{2,1}$-minimization problem can be formulated as

$$
\begin{array}{ll}
\min & \|\mathbf{Q X}\|_{2,1} \\
\text { subject to } & \|\mathbf{Y}-\mathbf{A X}\|_{F} \leqslant \epsilon .
\end{array}
$$

The WSF CS-based DOA estimation provides higher accuracy than the regular $\ell_{2,1^{-}}$ minimization since the weighting matrix $\mathbf{Q}$ can adjust the sparse penalty for each element in $\mathbf{X}$. WSF matrix does not require additional parameter settings in comparison with $\ell_{2,1}$-minimization, however it adds only one step to calculate the weighting matrix. More details can be found at Appendix A.3.1 and Appendix A.3.2.

\subsection{Riemannian Geometry-based DOA Estima- tion}

This section proposes a new DOA estimation technique based on Riemannian geometry (RG). The Riemannian geometry (RG)-based DOA estimation technique exploits the Riemannian mean of THPD covariance matrices and the Riemannian distance. The RGDOA estimation problem is formulated as a linear search optimization problem, where the estimated DOA is the reciprocal of the minimum Riemannian distance.

\subsubsection{Motivation}

DOA estimation is a principal task in array processing and radar signal processing since it is a precursor to beamforming, target localization and tracking. Several estimation techniques tackle the problem of estimating DOA for multiple sources by exploiting conventional methods like DAS and MVDR or subspace methods, such as MUSIC and ESPRIT or compressive sensing techniques like $\ell_{2,1}$ and WSF- $\ell_{2,1}$. These techniques can estimate DOAs for uncorrelated signals with high resolution [91]. However, the performance of these methods suffer degradation under a low SNR and small sample size [105].

DOA estimation based on information geometry exploits the geometry present in the space of probability distributions parametrized by their covariance matrix [106]. 
One aspect of information geometry focuses on considering probability density functions as structure of differential geometry and the geodesical distance, which is the shortest distance between two probability density functions. Specifically using Riemannian geometry [10], each density function parametrized by a Toeplitz Hermitian positive definite (THPD) covariance matrix represents a point on the statistical Riemannian manifold $[107,108]$. Using Karcher Barycenter [109], the Riemannian (geometric) mean of THPD covariance matrices can be calculated and the Riemannian distance can be defined by considering the curvature of the manifold.

The problem of DOA estimation is considered from a geometric point of view. In particular, a Riemannian geometry direction of arrival (RGDOA) estimation technique based on regularized Burg algorithm (RBA) in Sec. 2.4, the Riemannian mean and the Riemannian distance is proposed to maintain robust estimation under low SNR and small sample size. The RGDOA technique is formulated as an optimization problem by searching for the minimum Riemannian distance between the Riemannian mean and the steering vector Hermitian positive definite covariance matrices for all possible DOA angles. The solution for this optimization problem is a linear search for the minimum distance that corresponds to the DOA estimate.

\subsubsection{DOA Signal Model}

Let $L$ be the number of uncorrelated signals impinging from various directions given by $\boldsymbol{\theta}=\left[\theta_{1}, \ldots, \theta_{L}\right]$ on a uniform linear antenna array with $N$ elements. The received signals for the $k^{\text {th }}$ snapshot can be modeled as [110]

$$
\mathbf{z}[k]=\sum_{i=1}^{L} \mathbf{a}\left(\theta_{i}\right) \mathbf{s}_{i}[k]+\mathbf{n}[k]=\mathbf{A} \mathbf{s}[k]+\mathbf{n}[k]
$$

where $\mathbf{a}\left(\theta_{i}\right)=\left[1, \quad e^{-j 2 \pi \frac{d}{\lambda} \sin \left(\theta_{i}\right)}, \ldots, e^{-j 2 \pi(N-1) \frac{d}{\lambda} \sin \left(\theta_{i}\right)}\right]^{T}$ is the steering vector associated with the received signal at $\theta_{i}, d$ is the distance between two consecutive antennas, $\lambda$ is the wavelength, $\mathbf{A}=\left[\mathbf{a}\left(\theta_{1}\right), \ldots, \mathbf{a}\left(\theta_{L}\right)\right]$ is the array manifold matrix, $\mathbf{s}[k]=\left(s_{1}[k], \ldots, s_{M}[k]\right)$ is the received signal and $\mathbf{n}[k]$ is independent identically distributed additive complex Gaussian noise. The noise covariance matrix can be written as

$$
\mathbf{R}_{n}=\mathbb{E}\left[\mathbf{n}[k] \mathbf{n}^{H}[k]\right]=\operatorname{diag}\left(\sigma_{1}^{2}, \ldots, \sigma_{N}^{2}\right)
$$


where $\mathbb{E}$ is mathematical expectation and $\sigma_{i}^{2}$ is the noise variance of the $i^{\text {th }}$ antenna. The received signal covariance matrix can be defined as

$$
\mathbf{R}_{s}=\mathbb{E}\left[\mathbf{s}[k] \mathbf{s}^{H}[k]\right] .
$$

From (4.13), (4.14) and (4.15), it follows that the covariance matrix of $\mathbf{z}[k]$ can be expressed as

$$
\mathbf{R}_{z}=\mathbb{E}\left[\mathbf{z}[k] \mathbf{z}^{H}[k]\right]=\mathbf{A R}_{s} \mathbf{A}^{H}+\mathbf{R}_{n}
$$

which is the asymptotic covariance model.

\subsubsection{DOA Estimation in the Riemannian Space}

The idea of estimating the DOA in the Riemannian space is to consider the geometry present in the space of probability distributions parametrized by their covariance matrix. Each radar data snapshot is converted to a THPD covariance matrix which represents a point on the Riemannian manifold. These points can be manipulated on the Riemannian manifold using a Riemannian metric like the Riemannian distance. The Riemannian distance is based on the Fisher information matrix, which measures how close two distributions are. Using the Riemannian distance, the DOA estimation can be formulated as a linear search for the minimum Riemannian distance between steering vector HPD covariance matrices and the Riemannian mean.

The RBA in Sec. 2.4 is exploited to estimate the reflection coefficients for every snapshot of the radar observation data. From the reflection coefficients, we can estimate the THPD covariance matrix for each radar snapshot using (2.11). In addition, using (2.21), the Riemannian mean of the THPD covariance matrices residing on the Riemannian manifold can be computed. Also, we can generate an HPD covariance matrix for each of the steering vectors by using the following equation,

$$
\mathbf{R}_{\mathrm{sv}}\left(\theta_{i}\right)=\mathbf{a}\left(\theta_{i}\right) \mathbf{a}^{H}\left(\theta_{i}\right)+\gamma \mathbb{I}_{N \times N}, \quad \theta_{i} \in[-\pi / 2, \pi / 2]
$$

where $\gamma$ is the diagonal loading factor which is determined by the standard deviation of $\operatorname{diag}\left(\mathbf{a}\left(\theta_{i}\right) \mathbf{a}^{H}\left(\theta_{i}\right)\right)$ and $\mathbb{I}_{N \times N}$ is an $N \times N$ identity matrix.

The DOA estimation problem can be formulated as an optimization problem using 
(2.18), (2.21) and (4.17) by searching for the minimum Riemannian distance between the full rank $\mathbf{R}_{\mathrm{rm}}$ and full rank $\mathbf{R}_{\mathrm{sv}}(\theta)$.

The optimization problem can be expressed as [105]

$$
\begin{array}{ll}
\min & d_{\mathrm{R}}\left(\mathbf{R}_{\mathrm{rm}}, \mathbf{R}_{\mathrm{sv}}\left(\theta_{i}\right)\right) \\
\text { subject to } & \mathbf{R}_{\mathrm{rm}} \succ 0, \\
& \mathbf{R}_{\mathrm{sv}}\left(\theta_{i}\right) \succ 0, \theta \in[-\pi / 2, \pi / 2]
\end{array}
$$

where $\succ$ means positive definite matrix. The solution of the optimization problem in (4.18) is a linear search for the minimum distance $d_{\mathrm{R}}$. The reciprocal of $d_{\mathrm{R}}$ is associated with steering vector of the direction of arrival estimate.

\subsection{Simulation Results}

Two sets of simulation results are presented to evaluate the performance of the RGDOA technique in this section. The first set of simulation results are based on spatial spectrum presentation, root-mean-square error and closely spaced targets resolution. The second set of simulation results are based on investigating the effect of RSLA configuration on the Riemannian geometry-based DOA estimation. The RBA regularization parameter is set to $\gamma_{1}=0.01$ and the autoregressive order model is $N=8$. Also, the Riemannian mean step size is $\epsilon=0.01$ and the number of iterations is set to $t=1000$ with tolerance level set to $10^{-5}$. A collocated directional ULA is used with $M=8$ transmitters and $N=8$ receivers with the antenna elements spaced half a wavelength $\lambda$ apart from each other. The desired sources' DOAs are at $\theta=[-30,20]$ degrees. In addition, additive white Gaussian noise is considered with SNR of $10,0,-5$ and $-10 \mathrm{~dB}$ and the number of snapshots $K$ is $64,32,16$ and 8. The key point in this simulation is to measure the performance of the estimators as we decrease the SNR and the sample size. In addition, an omni-directional RSLA configuration with same size as the ULA and internal antenna elements spacing $[0.1 \lambda, 1.1 \lambda, 0.5 \lambda, 0.3 \lambda, 0.7 \lambda, 0.4 \lambda, 0.3 \lambda]$ is used to collect data at -15 degrees DOA. The goal of this simulation is to study the robustness of the RGDOA under RSLA configuration in comparison with ULA configuration.

The first simulation results evaluates the robustness of the Riemannian geometry DOA estimation technique for low SNR and small sample size. Figures 4.2 to 4.5 show the performance of DAS, MUSIC, MVDR and RGDOA for different sample 
sizes and SNRs. For $10 \mathrm{~dB}$ SNR and sample size $K=64$, we notice a similarity in performance for all DOA estimators, however, in terms of sidelobe levels, RGDOA outperformed the other DOA estimators. On the other hand, for $-10 \mathrm{~dB}$ SNR and sample size $K=8$ as shown in Fig. 4.5a, it can be noted that the performance of DAS, MUSIC and MVDR is significantly reduced in comparison with the RGDOA which still has robust estimates under low SNR and small sample size.

The second simulation results are based on root-mean-square error (RMSE) and Monte Carlo simulations to measure the DOA estimation performance of the RGDOA, WSF- $\ell_{2,1}, \ell_{2,1}$ and ESPRIT. The desired DOA source is at $\theta_{i}=-30$ degrees. The RMSE DOA estimation performance measure is based on

$$
R M S E=\sqrt{\frac{\sum_{i=1}^{N_{i}-1}\left\|\hat{\theta}_{i}-\theta_{i}\right\|_{2}^{2}}{M_{c}}}
$$

where $\theta_{i}$ is the vector of desired DOA sources, $\hat{\theta}_{i}$ is the DOA estimation and $M_{c}$ is the number of Monte Carlo experiments conducted. Figure 4.6, shows the performance of RGDOA, WSF- $\ell_{2,1}, \ell_{2,1}$ and ESPRIT for different sample sizes for $\mathrm{SNR}=0 \mathrm{~dB}$. The RGDOA maintained a robust performance at high and low sample sizes while the WSF- $\ell_{2,1}, \ell_{2,1}$ and ESPRIT suffer degradation (high DOA estimation RMSE) at low sample sizes. Additionally, the DOA estimation errors for different SNRs and $K=32$ snapshots, are provided in Fig. 4.7. The RGDOA outperformed WSF- $\ell_{2,1}, \ell_{2,1}$ and ESPRIT at lower SNR and maintained a robust superior performance at higher SNR.

The third simulation results demonstrate the performance of the proposed RGDOA, DAS, MUSIC and MVDR for closely spaced targets environment under low SNR and small sample size. Figure 4.8 shows the spatial spectrum of the RGDOA, DAS, MVDR and MUSIC for different spacing between closely spaced targets for 16 snapshots at $10 \mathrm{~dB}$ SNR. At 5 degrees spacing, all the techniques failed to resolve the two targets. However, as we increase the spacing between closely spaced targets, the MUSIC and MVDR techniques began resolving the two targets at 8 degrees. On the other hand, RGDOA began resolving the two targets at 12 degrees spacing as 
shown in Fig. 4.8d. As we decrease the SNR to $-10 \mathrm{~dB}$ and reduce the number of snapshots to 8, DAS, MVDR and MUSIC failed to resolve the closely spaced two targets as shown in Fig. 4.9. On the other hand, RGDOA began resolving the two closely spaced targets at 12 degrees as shown in Fig. 4.9d. Although RGDOA requires at least 12 degree of spacing to resolve two closely spaced targets at high SNR, it maintained a robust performance under low SNR and small small size, while the other techniques suffered performance degradation.

The next set of simulation results studies the performance of the RGDOA technique for RSLA configurations. Varying the spacing between antenna elements affects the eigenvalues of the estimated covariance matrices, hence affecting the Riemannian distance between the Riemannian mean and each of the THPD covariance matrices residing on the Riemannian manifold. Figure 4.10 shows the RGDOA estimates for 16 snapshots for different SNRs for ULA and RSLA configurations. At high SNR, we can notice a difference between RGDOA for ULA and RGDOA for RSLA. However, as we decrease the SNR, the DOA difference between ULA and RSLA is minimized. The performance of RGDOA for ULA and RSLA configurations can be measured using RMSE in 4.19. Figure 4.11 demonstrates the RMSE performance of RGDOA for ULA and RSLA configurations for different SNR values. We notice that at low SNR, the RGDOA estimation technique has similar performance for ULA and RSLA configurations. On the other hand, at higher SNR, we can see differences in performance between ULA and RSLA.

The random spacing between antenna elements in RSLA not only affects the propagation delay between antenna elements, but also varies the phase center of the antenna array, which degrades the DOA estimation performance for high SNR. In addition, ULA and RSLA suffer from ambiguities among the direction of arrival of the targets, for example the targets' angles at 0 and 180 degrees affect the steering vectors and manifold array matrix and lead to poor DOA estimation. The effect of phase center variation will be explored in future work. 


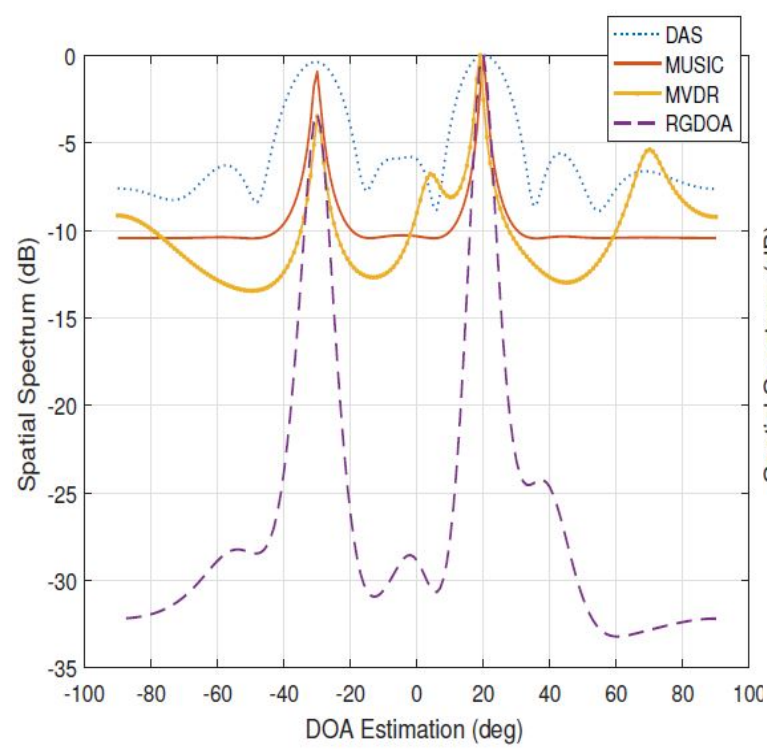

(a) $K=8$

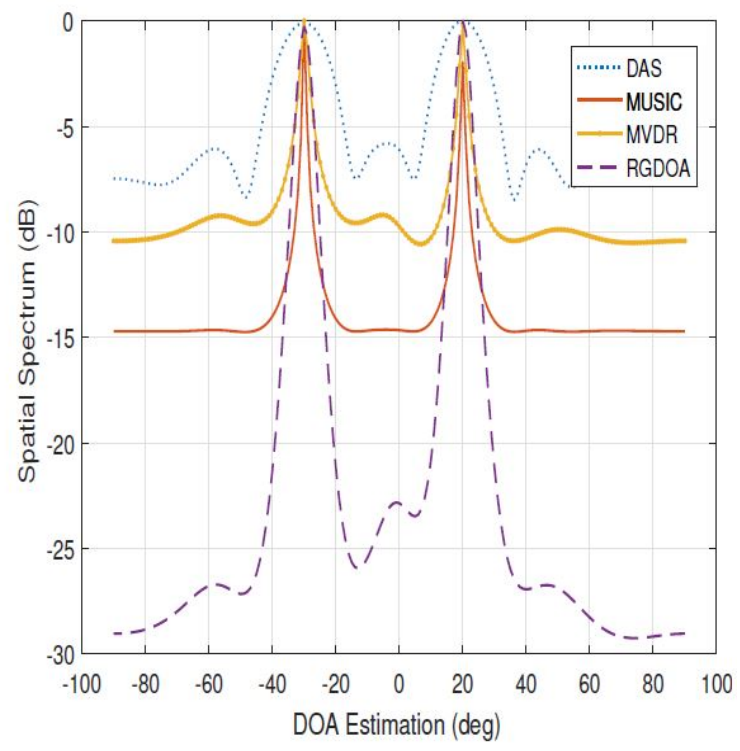

(c) $K=32$

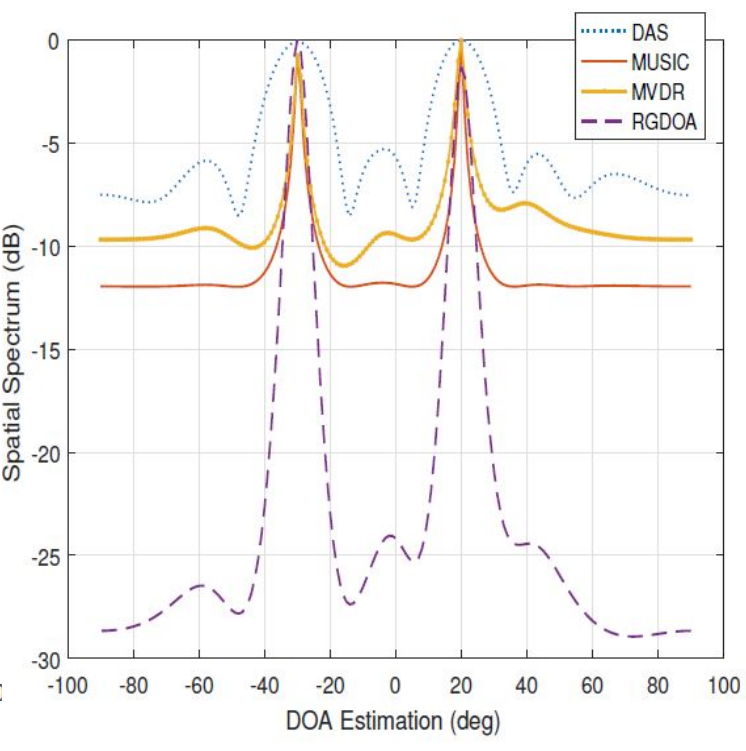

(b) $K=16$

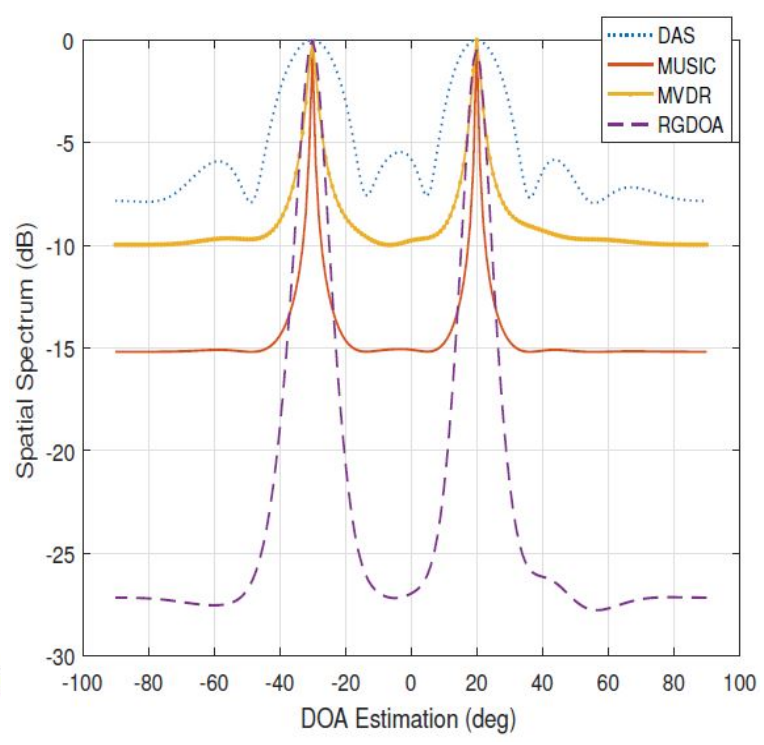

(d) $K=64$

Figure 4.2: Spatial spectrum for RGDOA, DAS, MVDR and MUSIC for SNR=10 $\mathrm{dB}$. 


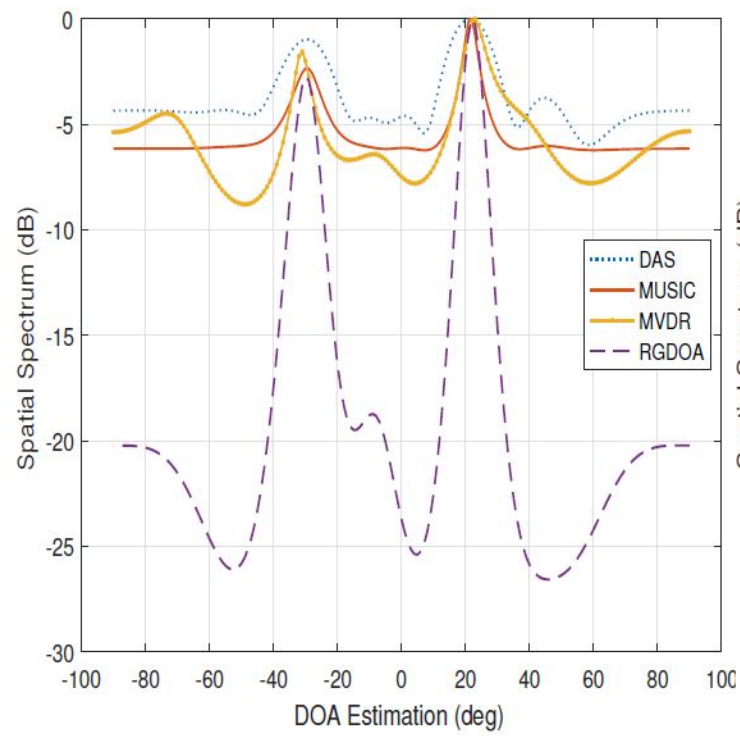

(a) $K=8$

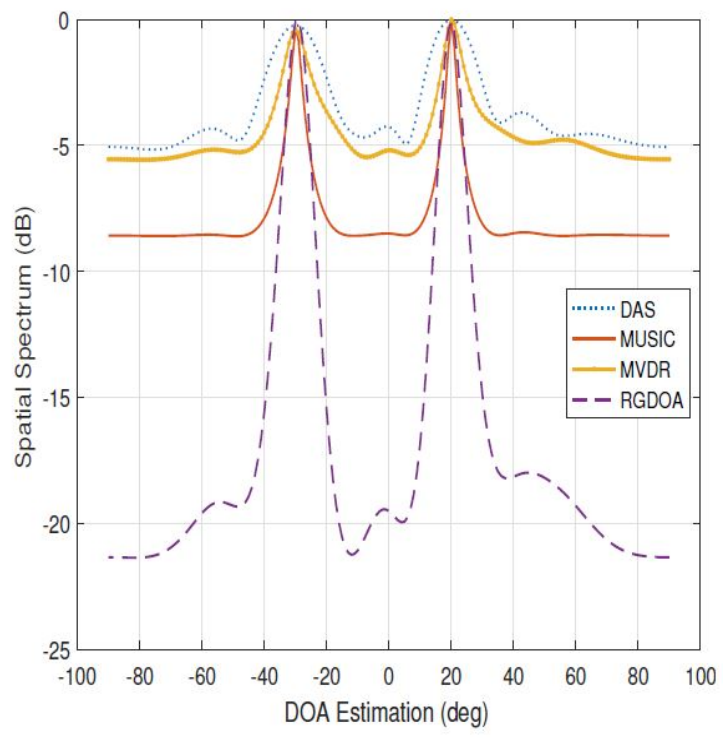

(c) $K=32$

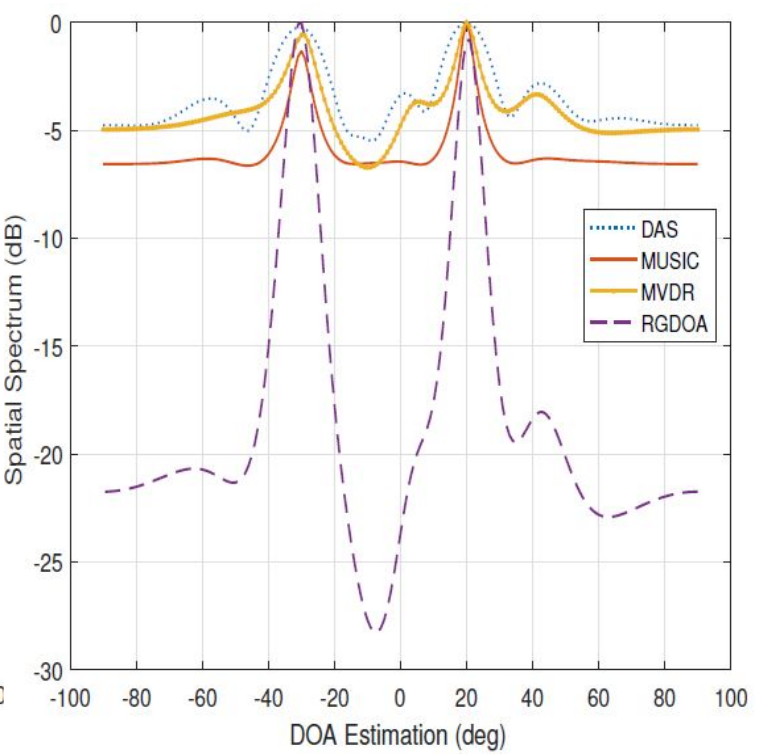

(b) $K=16$

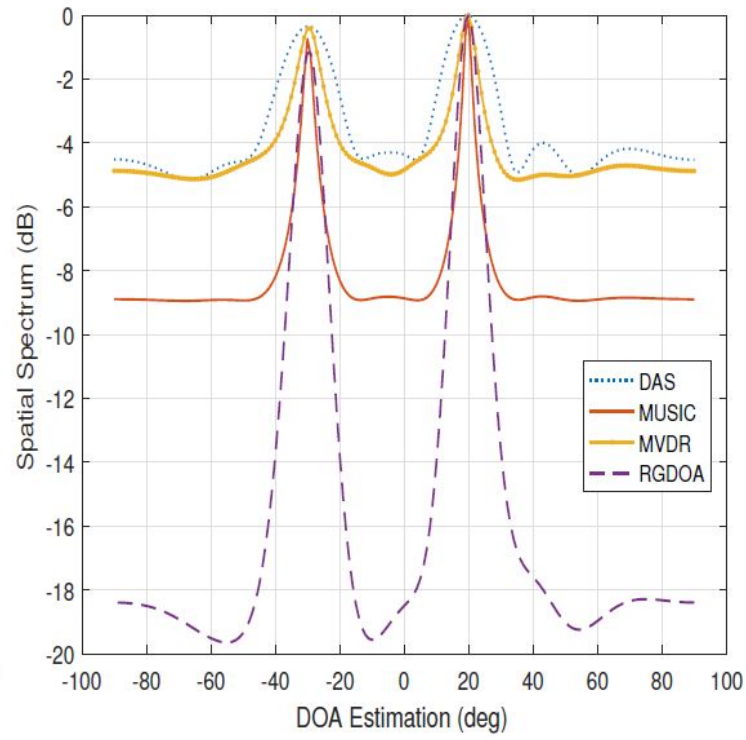

(d) $K=64$

Figure 4.3: Spatial spectrum for RGDOA, DAS, MVDR and MUSIC for $\mathrm{SNR}=0$ $\mathrm{dB}$. 


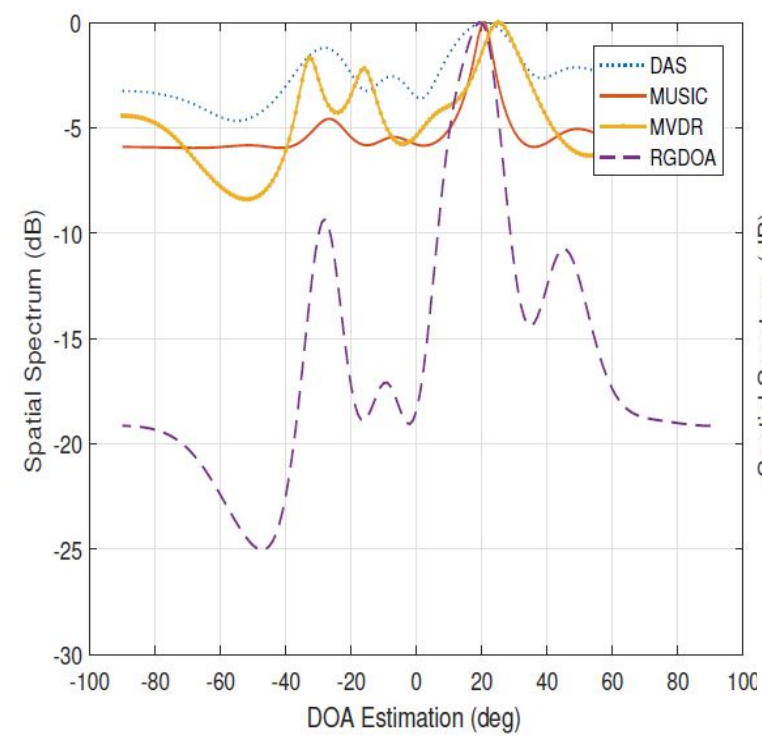

(a) $K=8$

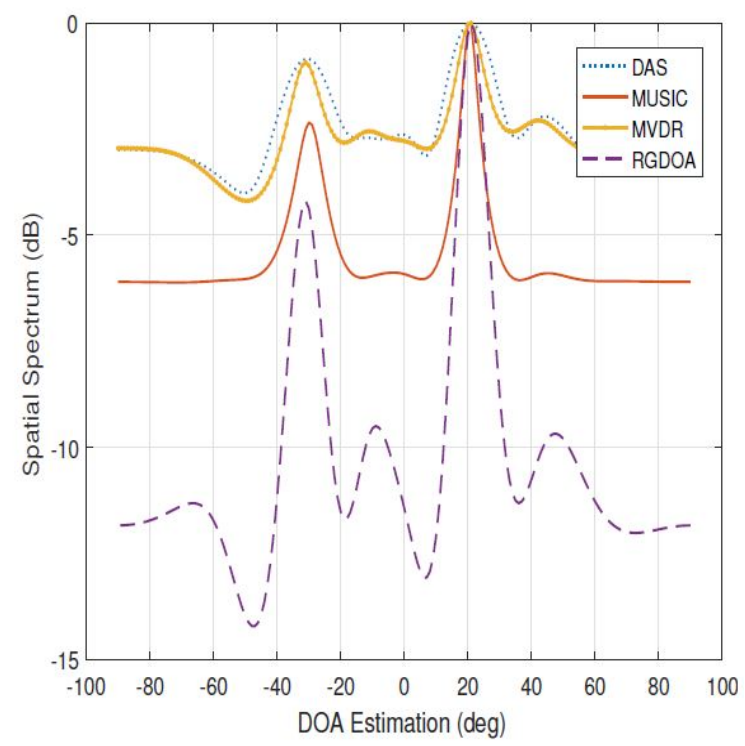

(c) $K=32$

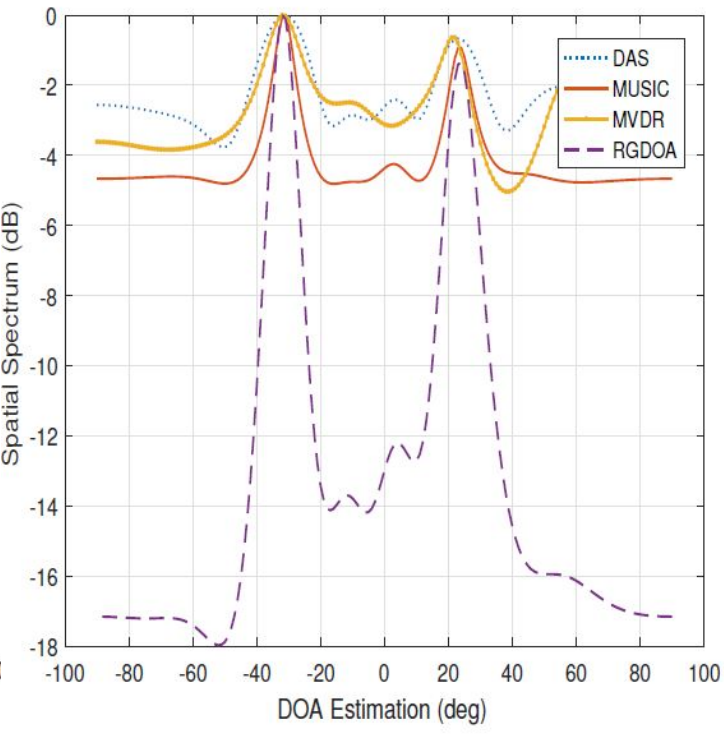

(b) $K=16$

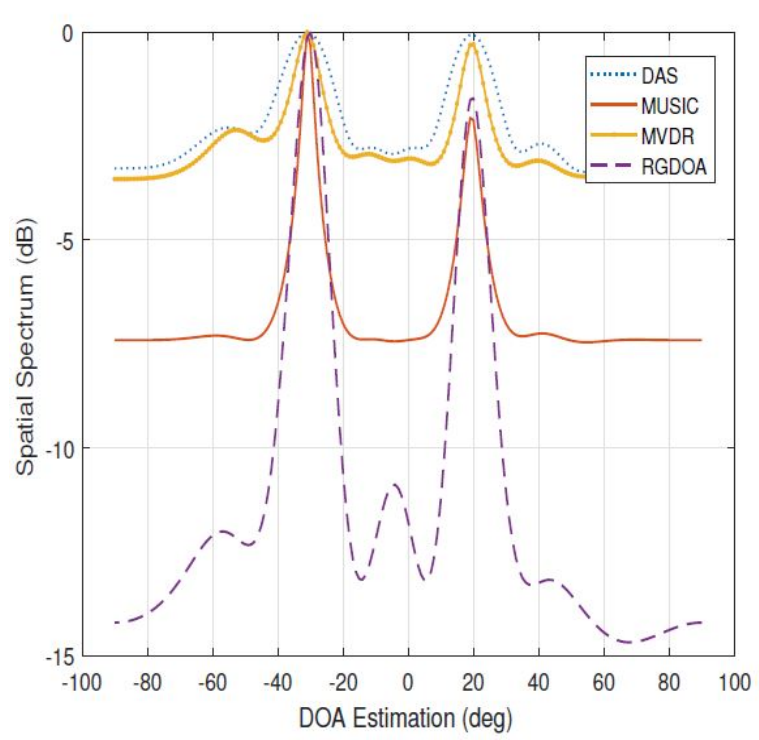

(d) $K=64$

Figure 4.4: Spatial spectrum for RGDOA, DAS, MVDR and MUSIC for SNR=-5 $\mathrm{dB}$. 


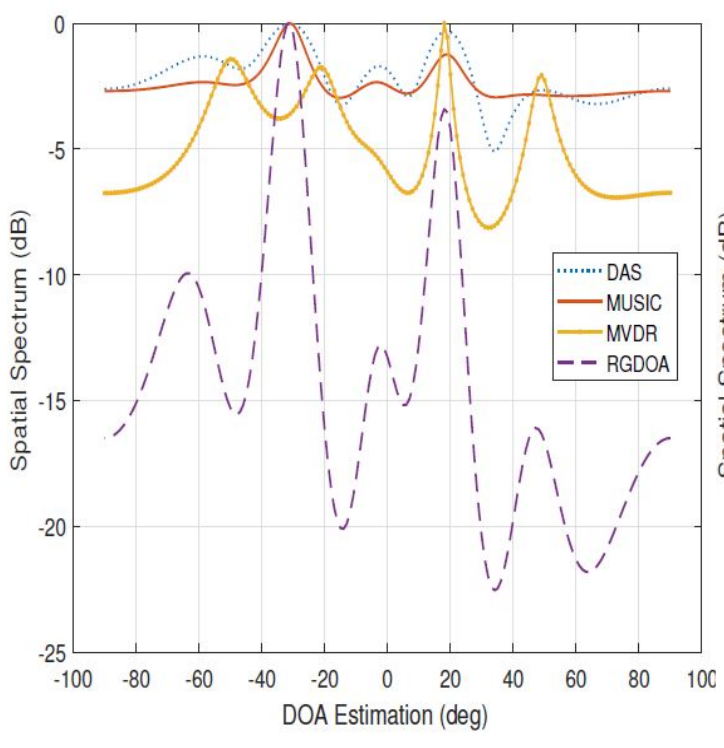

(a) $K=8$

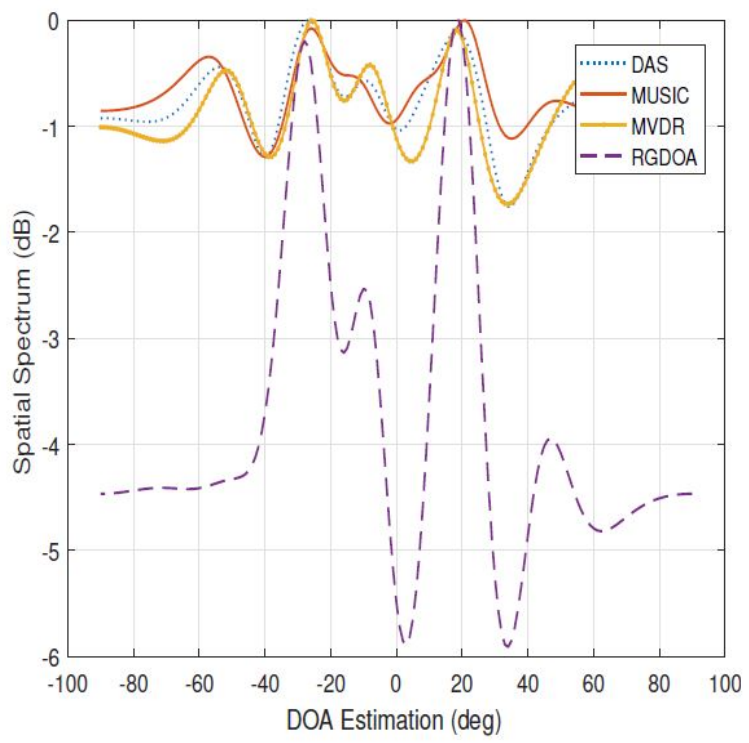

(c) $K=32$

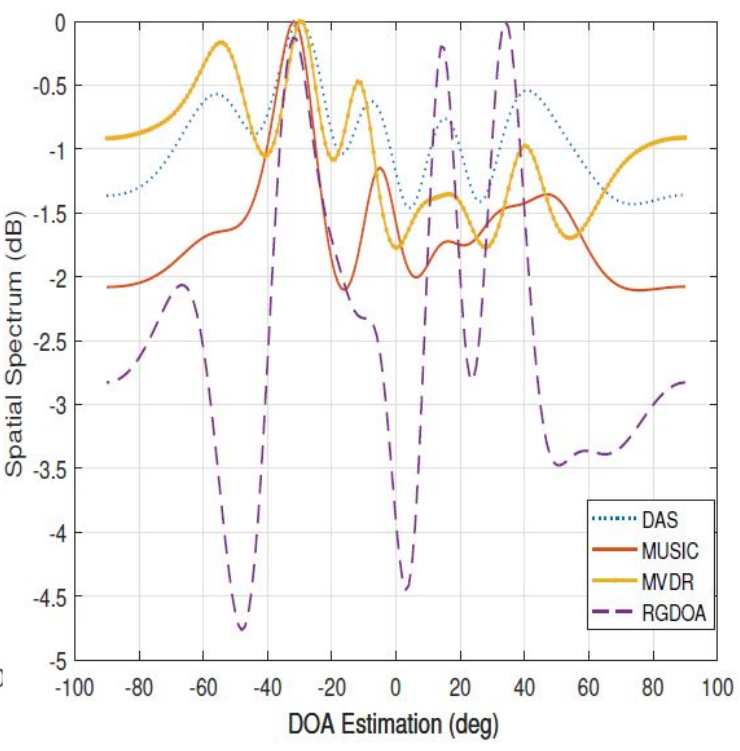

(b) $K=16$

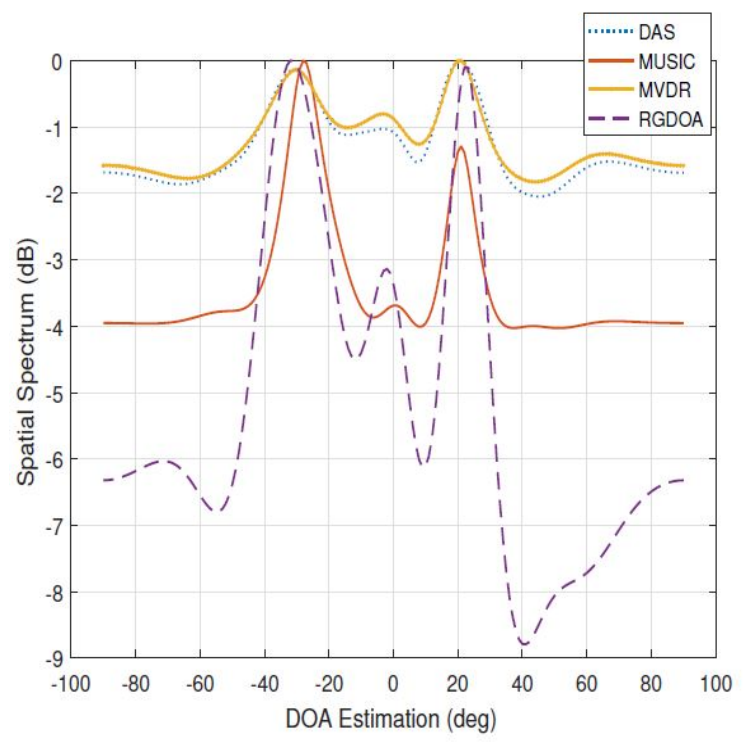

(d) $K=64$

Figure 4.5: Spatial spectrum for RGDOA, DAS, MVDR and MUSIC for SNR=-10 $\mathrm{dB}$. 


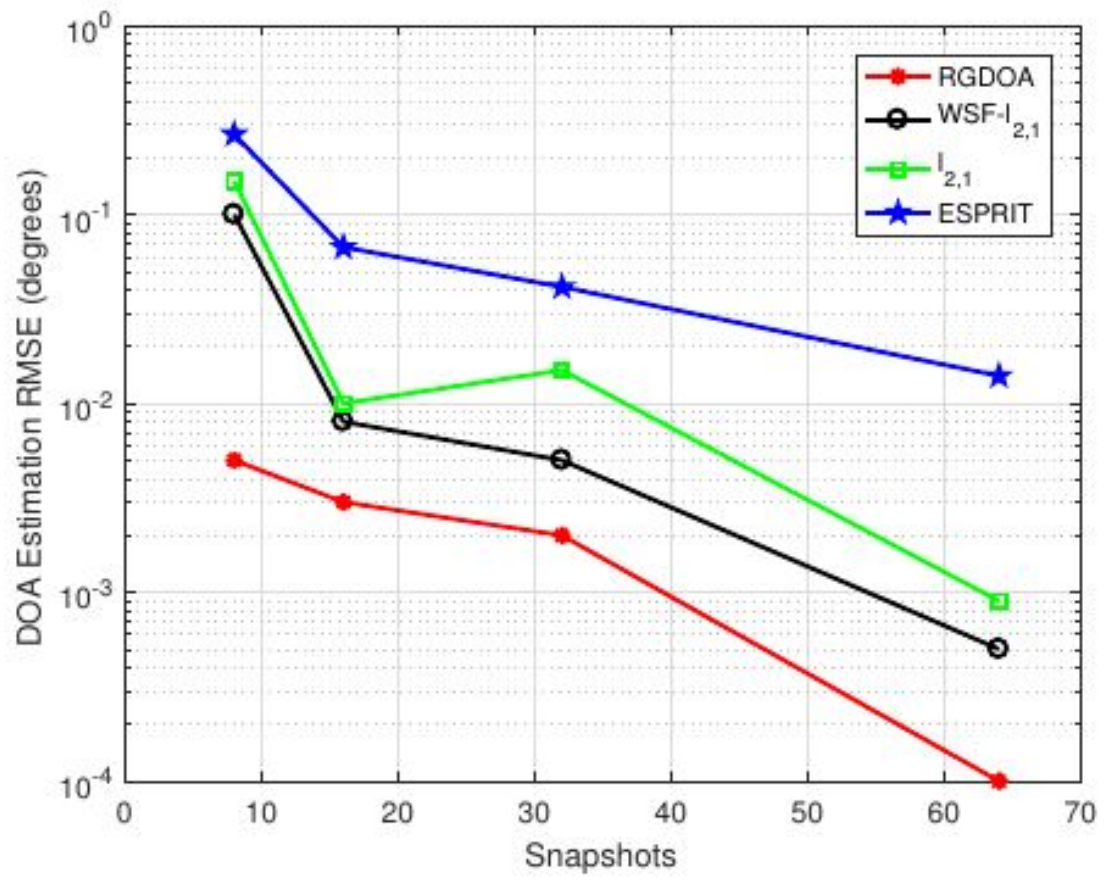

Figure 4.6: DOA estimation with different sample sizes at $\mathrm{SNR}=10 \mathrm{~dB}$.

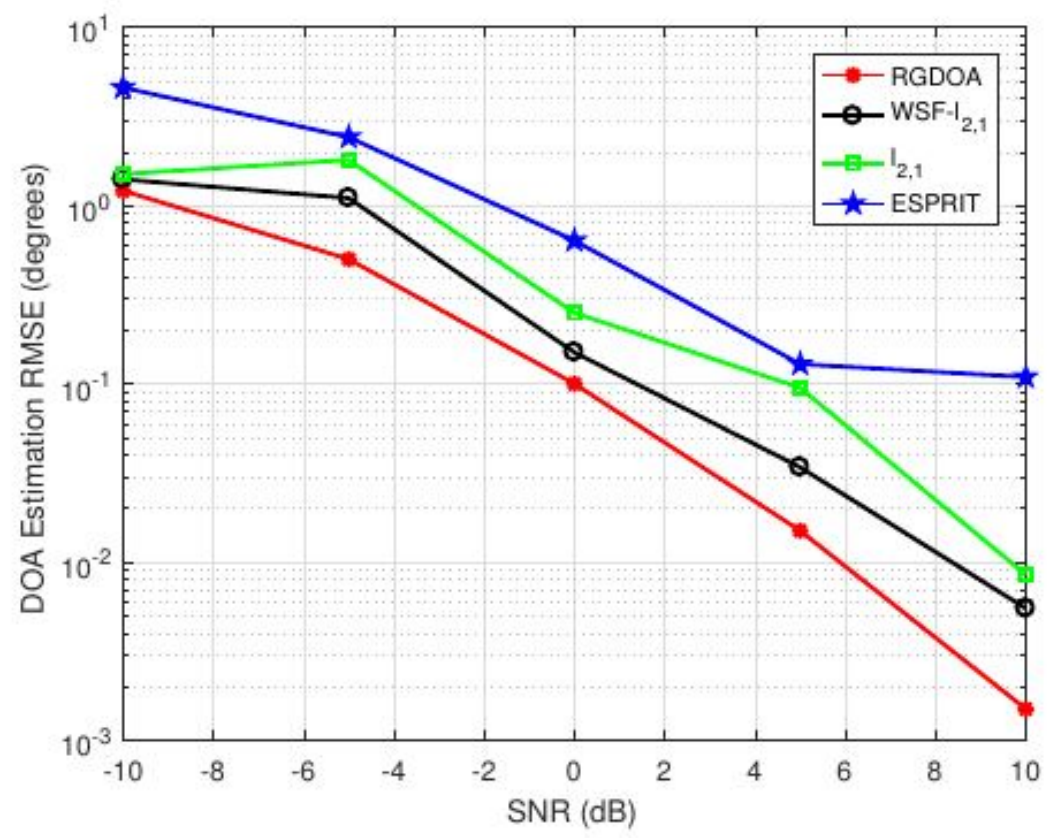

Figure 4.7: DOA estimation with different $\mathrm{SNR}$ at $\mathrm{K}=32$. 


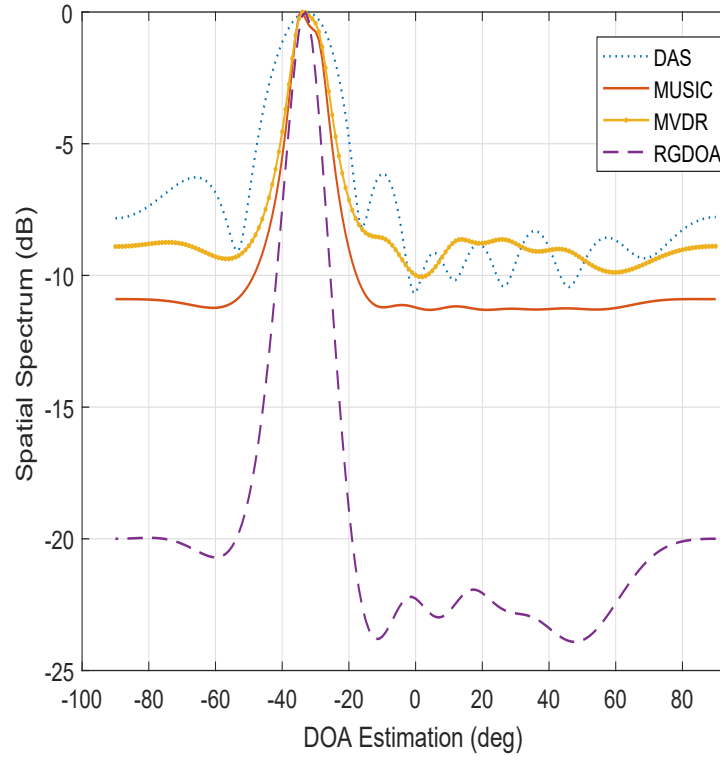

(a) Taroets snarino 5 deorees

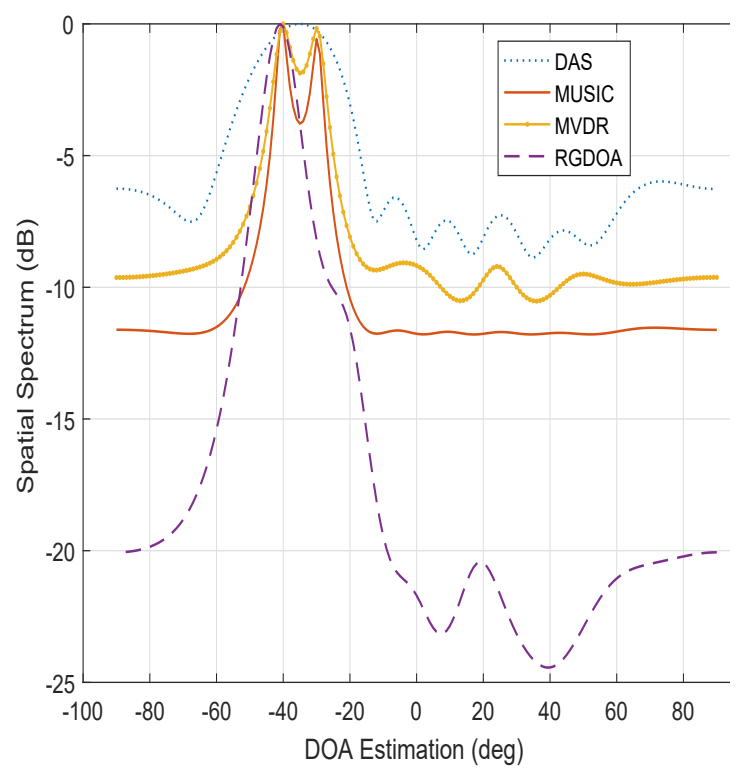

(c) Targets spacing 10 degrees

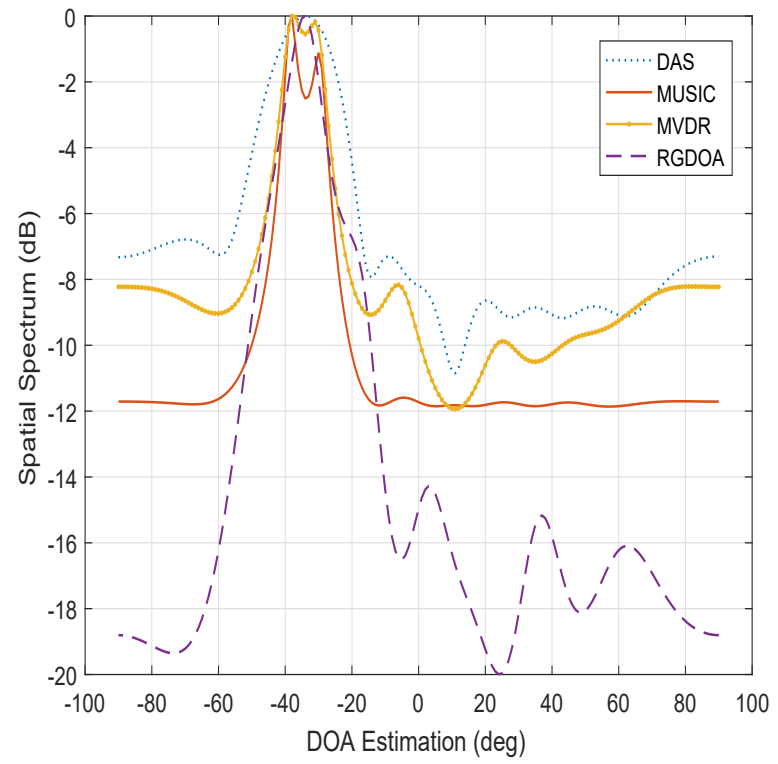

(h) Taroets snarino 8 deorees

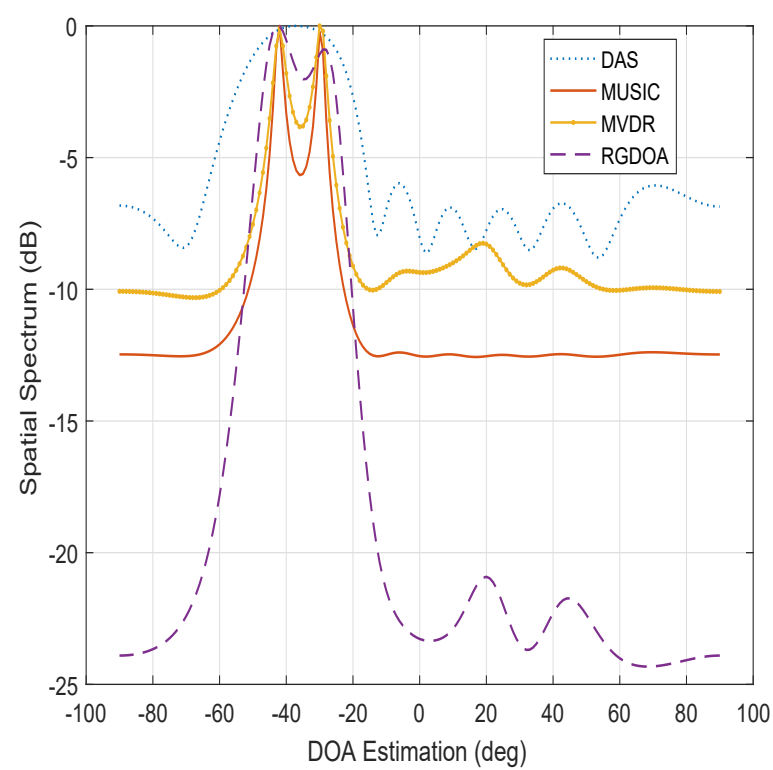

(d) Targets spacing 12 degrees

Figure 4.8: Spatial spectrum for RGDOA, DAS, MVDR and MUSIC for different closely spaced targets at $10 \mathrm{~dB}$ SNR and 16 snapshots. 


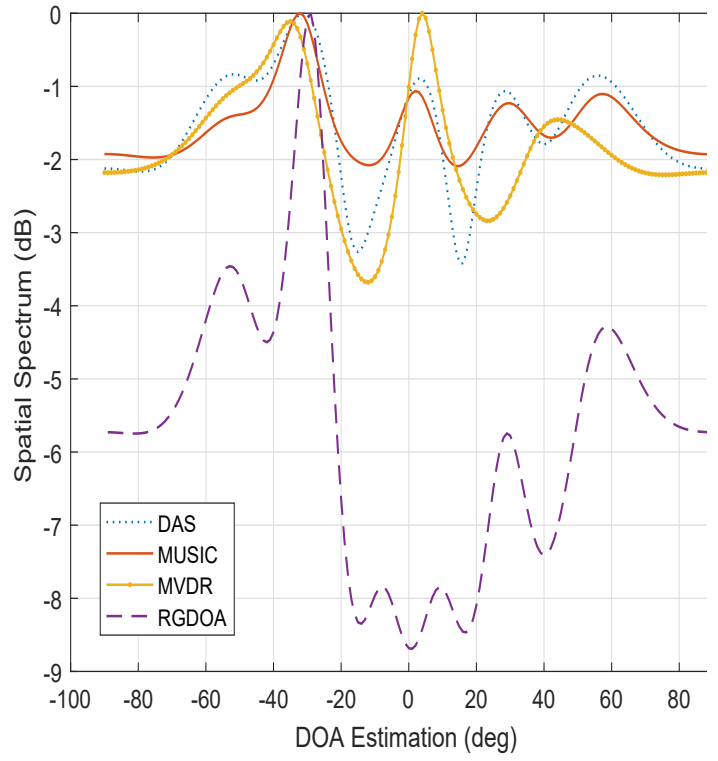

(a) Taroets snarino 5 deorees

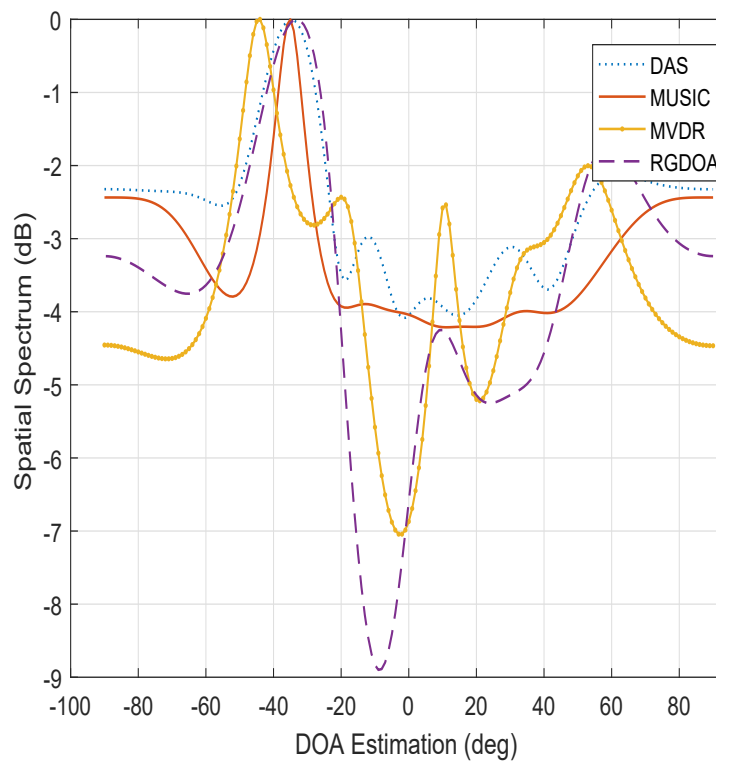

(c) Targets spacing 10 degrees

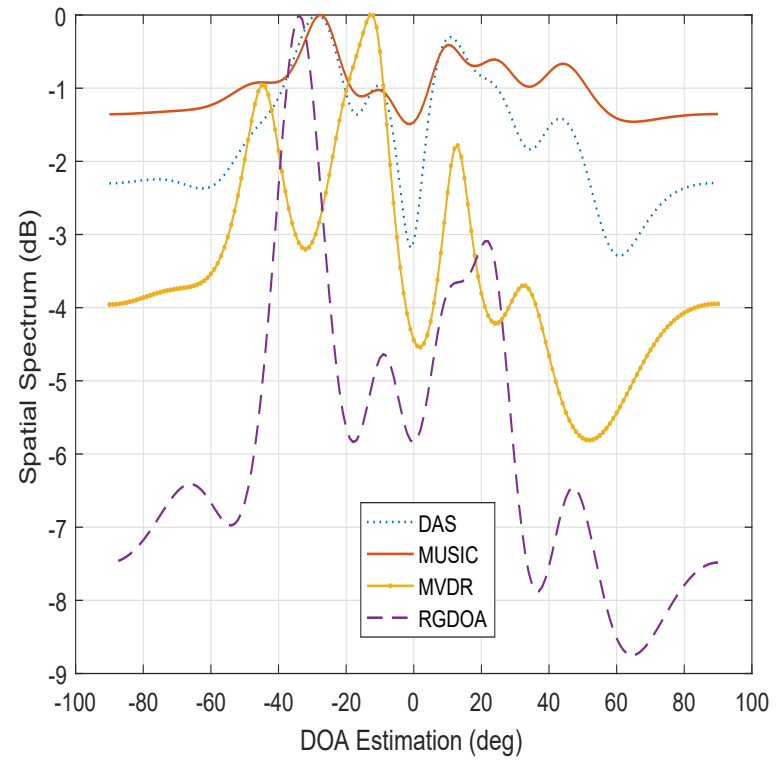

(h) Taroets snarino 8 deorees

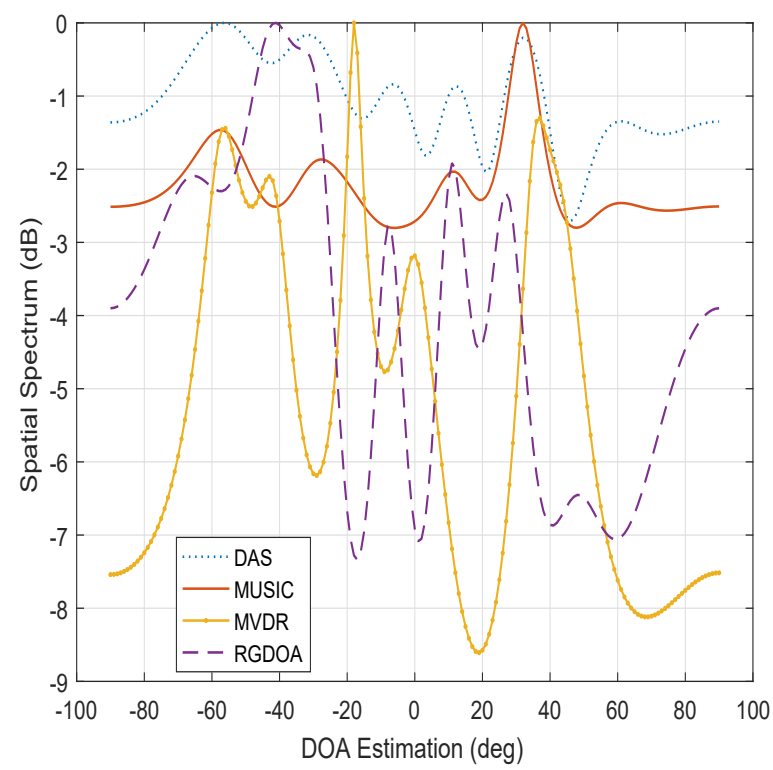

(d) Targets spacing 12 degrees

Figure 4.9: Spatial spectrum for RGDOA, DAS, MVDR and MUSIC for different closely spaced targets at $-10 \mathrm{~dB}$ SNR and 8 snapshots. 


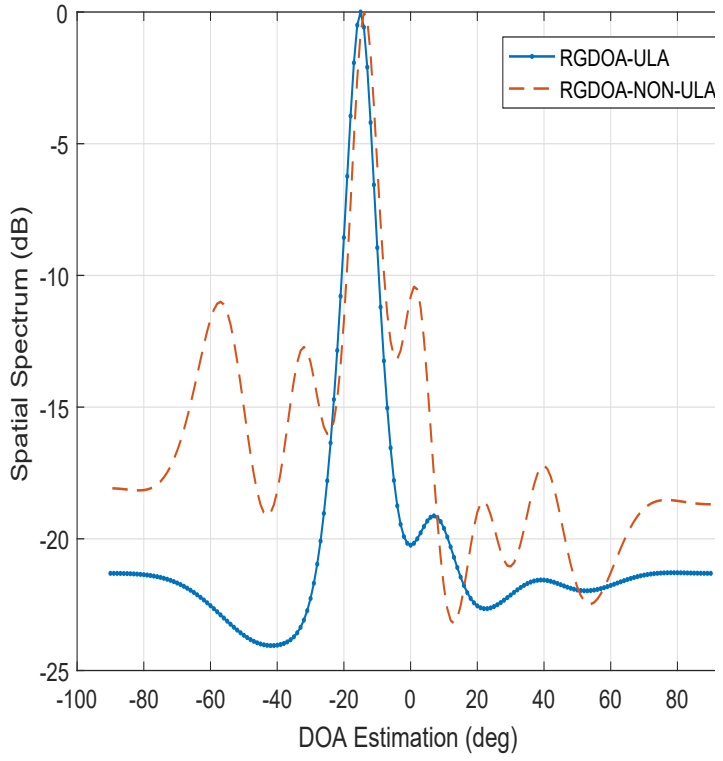

(a) $10 \mathrm{dR}$ SNR

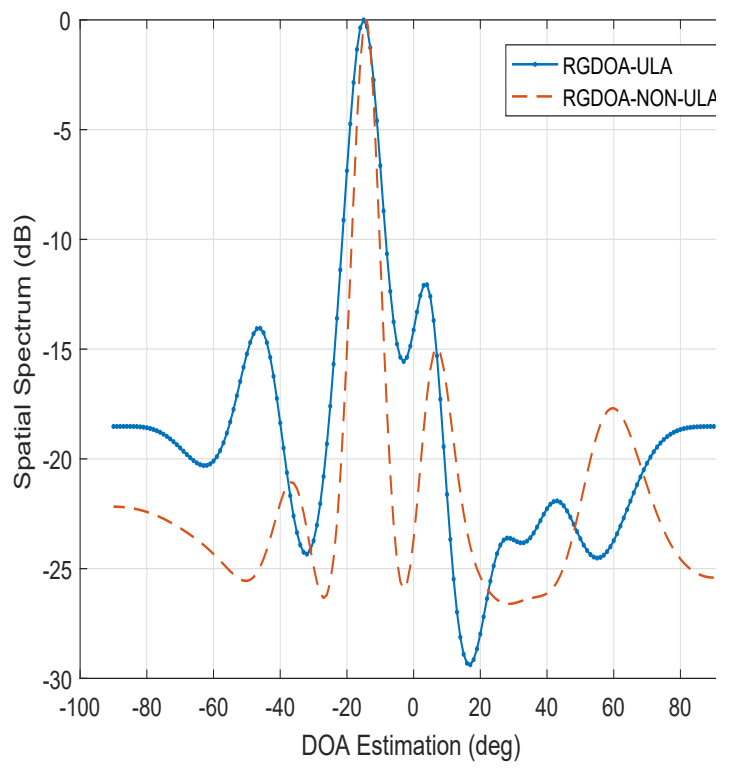

(c) $0 \mathrm{~dB}$ SNR

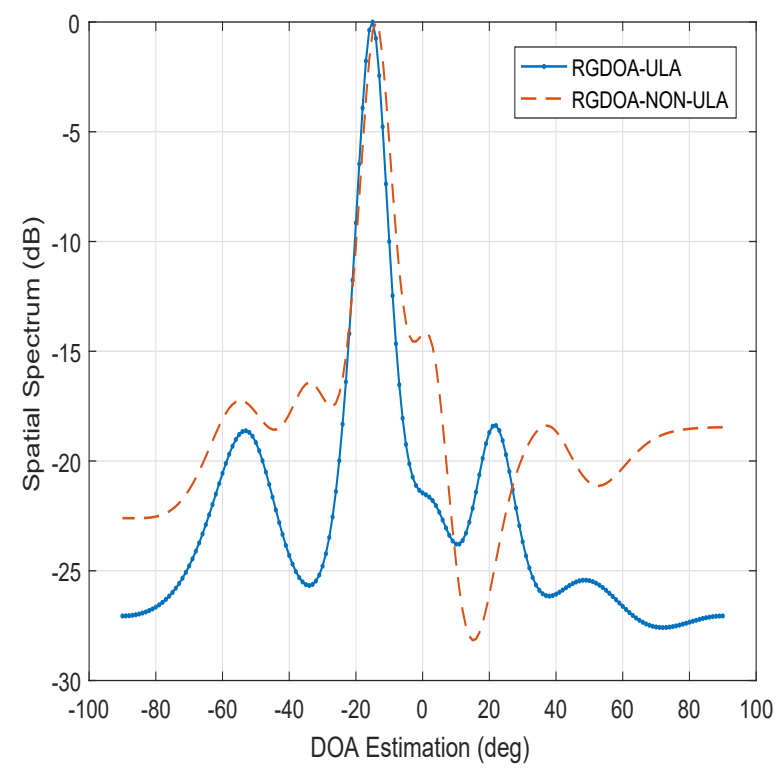

(h) $5 \mathrm{dR}$ SNR

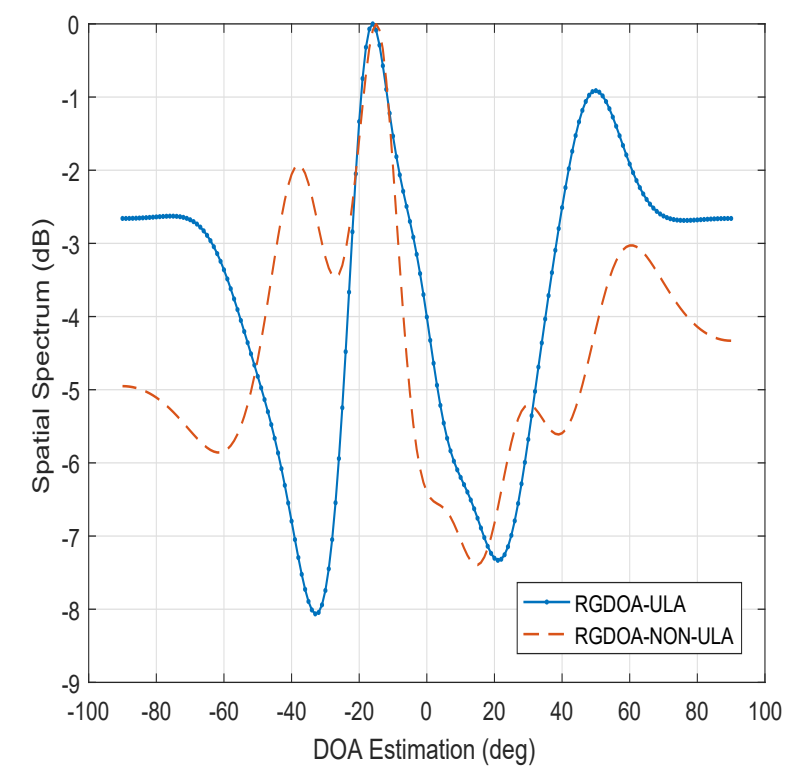

(d) $-10 \mathrm{~dB}$ SNR

Figure 4.10: Spatial spectrum of RGDOA for ULA and RSLA configurations for 16 snapshots under different SNRs. 


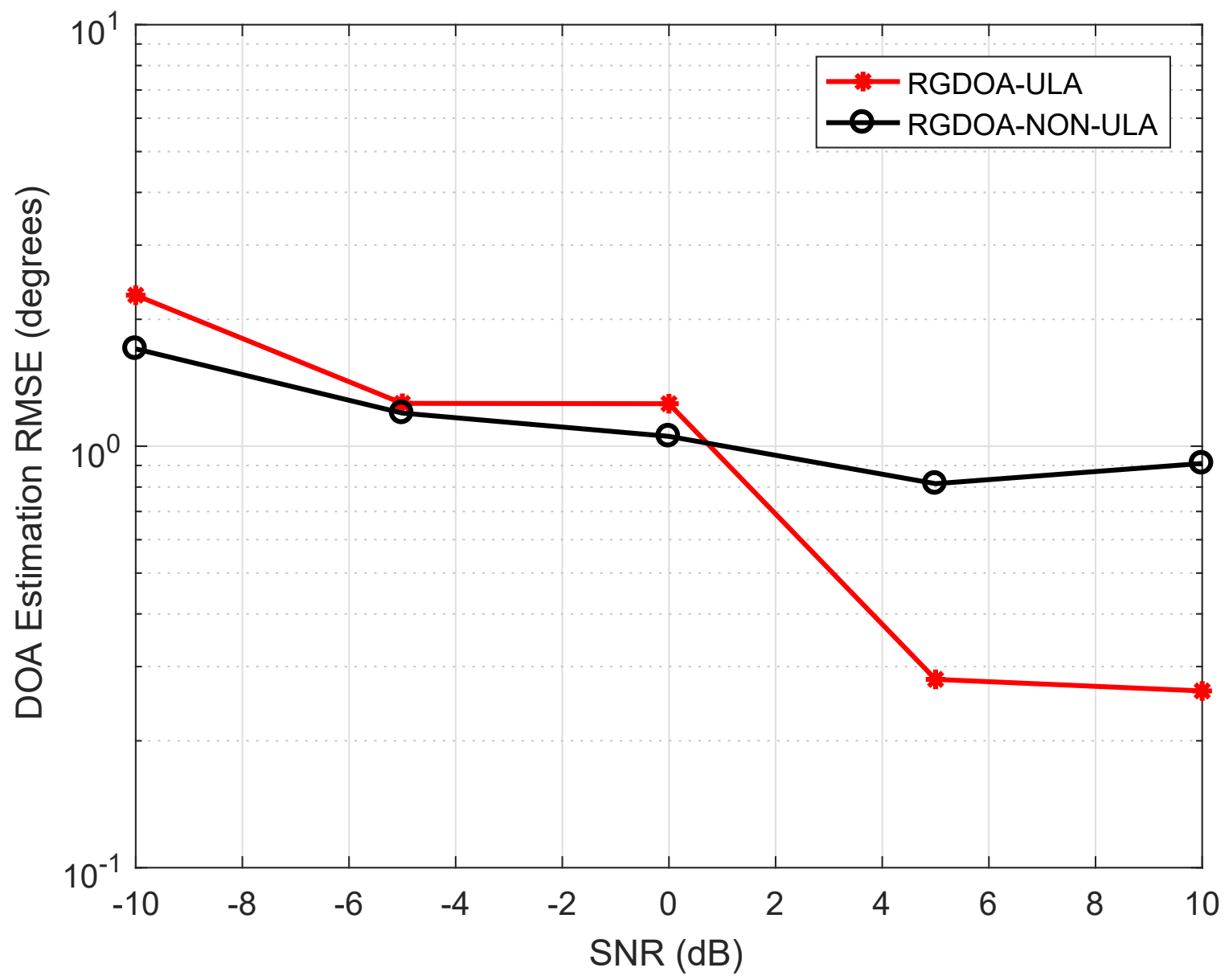

Figure 4.11: RMSE between true DOA and the RGDOA estimates for ULA and RSLA configurations for 8 snapshots for different SNRs. 


\subsection{Real Data Analysis}

The performance of the RGDOA technique is tested on real data from a drone experiment. The data was collected using the Texas Instruments uniform linear array AWR1642 automotive multiple input multiple output radar with a moving Yuneec Typhoon H drone target. The AWR1642 specifications are listed in Table 3.1.

The performance of the proposed technique is tested on $K=8,16,32$ and 64 snapshots extracted from the data set and compared to the DAS, MUSIC and MVDR DOA techniques. The analysis is based on the spatial spectrum of DAS, MUSIC, MVDR and RGDOA. The extracted snapshots are converted to THPD covariance matrices using RBA in Sec. 2.4. The Riemannian mean is calculated using all the THPD covariance matrices with regularization parameter set to $\gamma_{1}=0.1$ and the autoregressive order model is $N=4$. Also, the Riemannian mean step size is $\epsilon=$ 0.01 and the number of iterations is set to $t=1000$ with tolerance level set to $10^{-5}$. The Riemannian distance is computed between the Riemannian mean and each THPD covariance matrix residing on the Riemannian manifold. As we can see from Fig. 4.12, all techniques maintained good performance in estimating the DOA since the original data has high SNR. However, the RGDOA maintained low sidelobe levels in comparison with the other techniques.

The range of the drone can be extended by increasing the noise level of the original signal (scale the SNR by 0.2) to test the performance of the RGDOA in comparison with the other techniques. Figure 4.13 illustrates the DOA estimation performance of DAS, MUSIC, MVDR and RGDOA. The RGDOA technique maintained a robust estimate for different snapshot sizes, while DAS, MUSIC and MVDR suffered degradation in performance. 


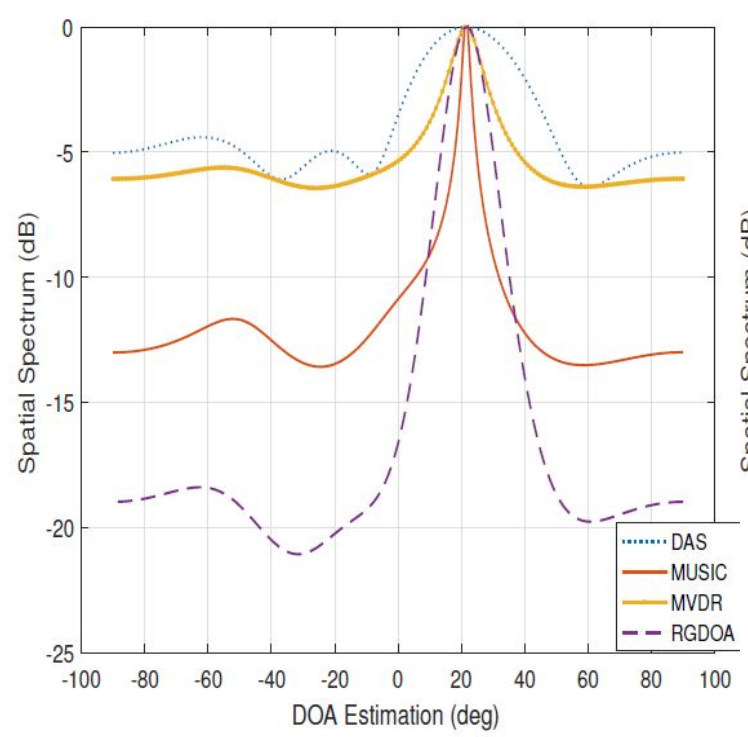

(a) $K=64$

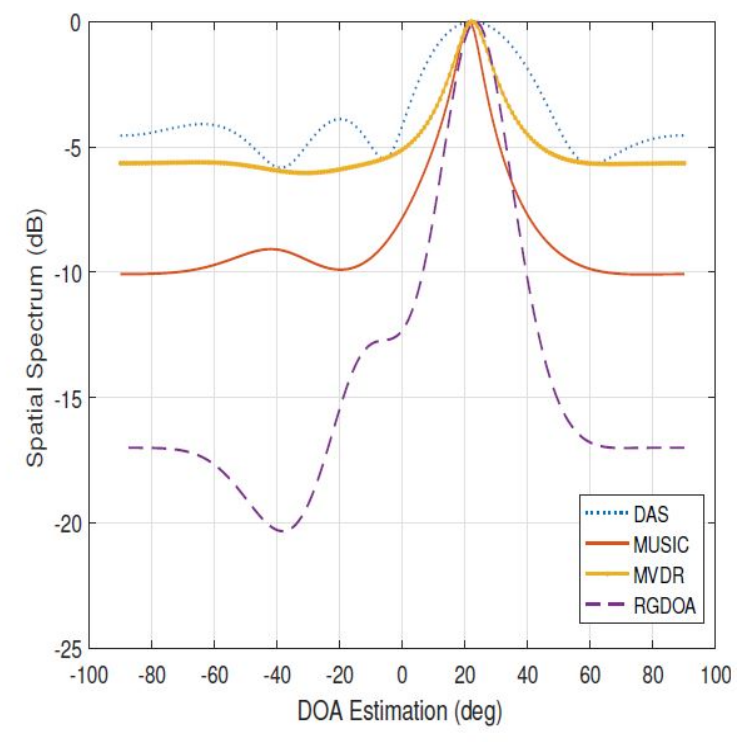

(c) $K=16$

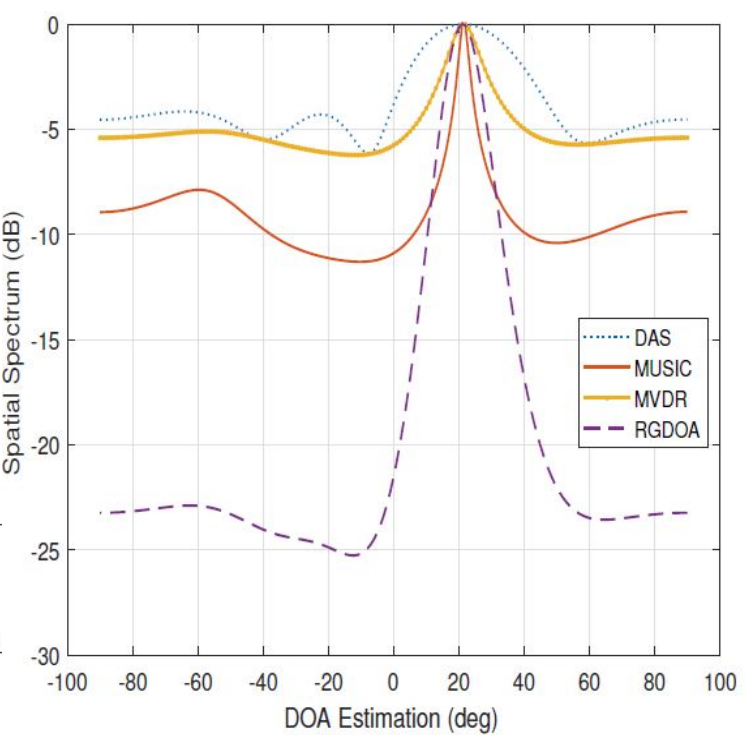

(b) $K=32$

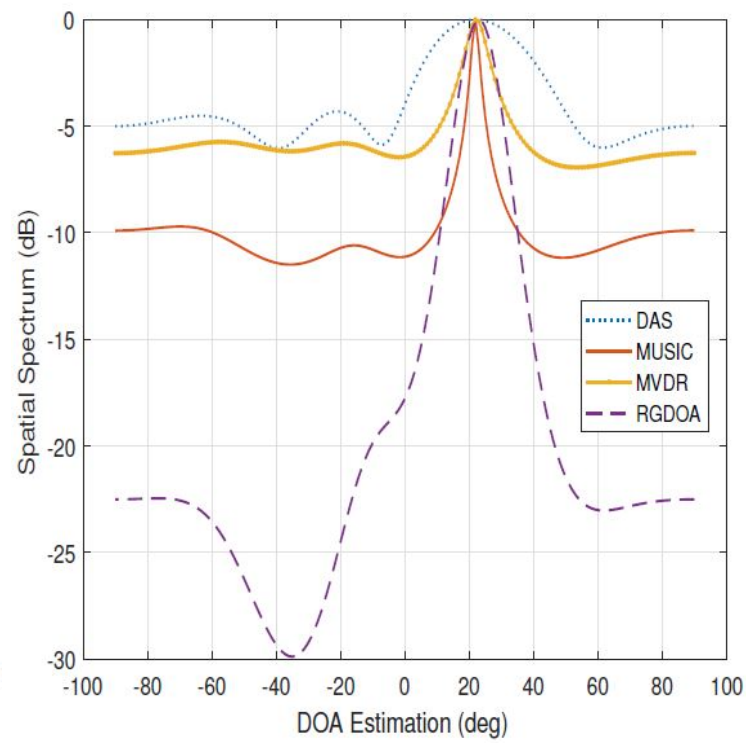

(d) $K=8$

Figure 4.12: Spatial spectrum for RGDOA, DAS, MVDR and MUSIC for original data. 


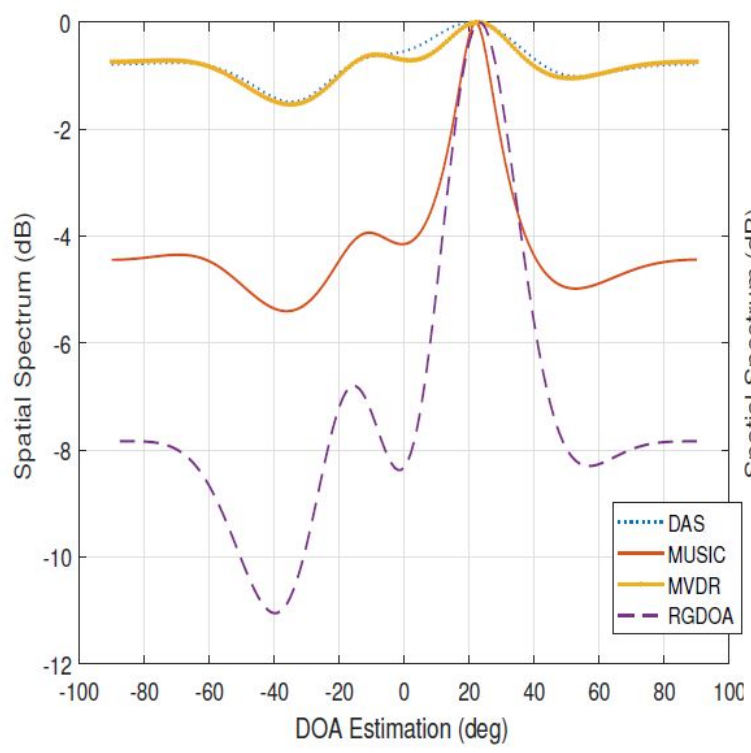

(a) $K=64$

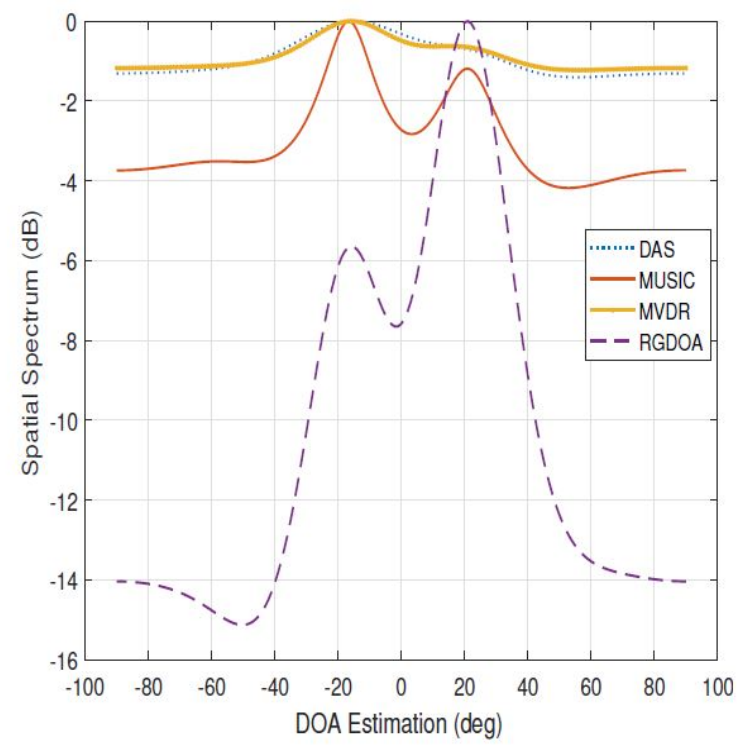

(c) $K=16$

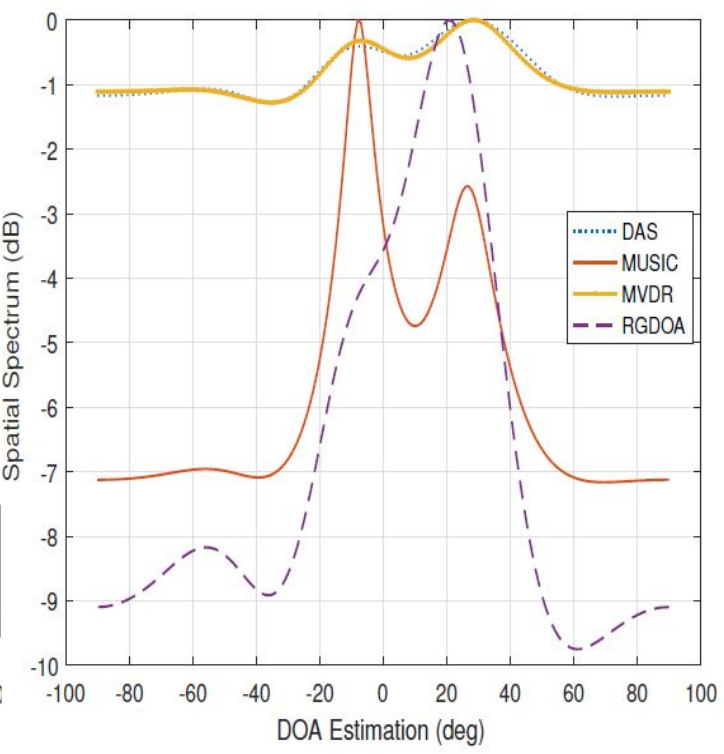

(b) $K=32$

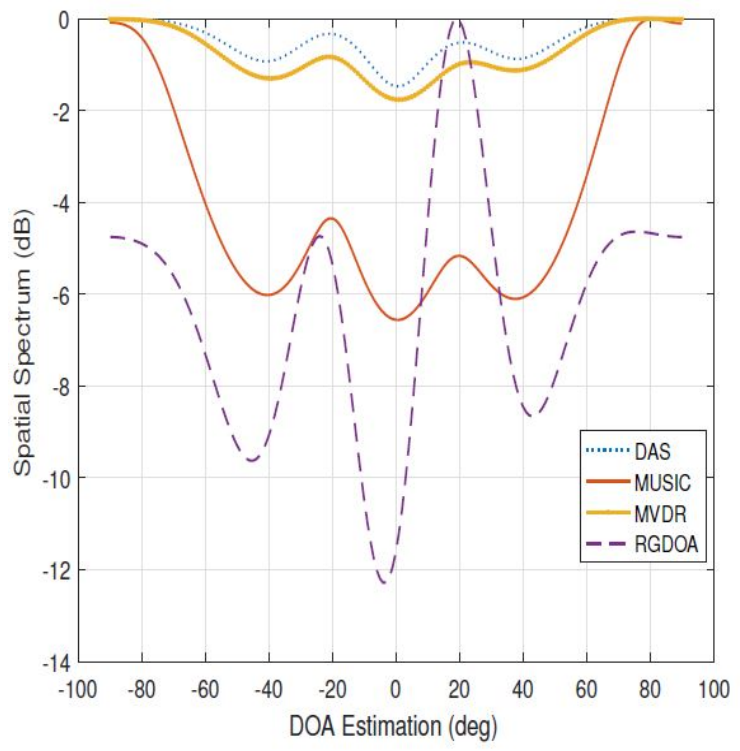

(d) $K=8$

Figure 4.13: Spatial spectrum for RGDOA, DAS, MVDR and MUSIC for scaled SNR. 


\subsection{Conclusion}

DOA estimation is a fundamental problem in a variety of applications including radar, sonar and wireless communications. Many different algorithms, such as DAS, MVDR, MUSIC, ESPRIT and compressive sensing techniques provide solution to DOA estimation problem. Most of these non-Riemannian techniques fail under small sample size and low SNR. The DOA estimation problem is projected into the Riemannian space where Riemannian distance and Riemannian mean can be exploited to formulate the DOA estimation problem as an optimization model. The solution of this optimization problem is a linear search for the minimum distance between the Riemannian mean and THPD for each steering vector. Simulation results and real data analysis showed that RGDOA maintained similar performance to DAS, MVDR, MUSIC, WSF- $\ell_{2,1}, \ell_{2,1}$ and ESPRIT at high SNR and large sample size and outperformed them at low SNR and small sample size. The RGDOA estimation for RSLA configuration has similar performance to ULA configuration at low SNR. On the other hand, at high SNR, the RGDOA for RSLA suffered slight degradation in performance in comparison with RGDOA estimation under ULA. 


\section{Chapter 5}

\section{Covariance Matrix Estimation for Beamforming Applications}

Adaptive beamforming has many applications in radar, sonar, seismology, microphone array speech processing, and, more recently, multiple input multiple output wireless communication $[85,111]$. As a major player in array processing, adaptive beamforming acts like a spatial filter that controls the beamforming weights to increase the signal strength while eliminating interference and noise. Unlike a classical conventional data-independent beamformer, adaptive beamforming relies on the received data to optimize the antenna array and achieve maximum gain in the direction of the desired signal while suppressing the interference directions [112]. Nevertheless, an adaptive beamformer like the MVDR algorithm is sensitive to model mismatch especially when the covariance matrix contains the desired target. For example, the estimated sample covariance matrix may have inaccuracy due to limited number of snapshots or training data. Also, another important challenge in adaptive beamforming is to maintain its performance even in the presence of uncertainty due to mismatch between the actual and the presumed steering vectors.

The estimation of the interference-plus-noise covariance matrix is a crucial step towards improving the performance of adaptive beamforming, as it leads to an increase in the target's signal-to-noise ratio and provides immunity against interferences. The sample covariance matrix, which is the maximum likelihood estimate of the data covariance matrix, does not always yield the desired estimation accuracy, especially with a small sample size, which leads to significant performance degradation [113].

This chapter provides brief summaries of the major techniques for estimating the 
interference-plus-noise covariance matrix, such as diagonal loading [114], shrinkage algorithm [115], and subspace [116] and sparse [117] techniques. In addition, it proposes a new Riemannian geometry-based interference-plus-noise covariance matrix estimation technique. This technique takes advantage of the Riemannian mean, the Riemannian distance and max-min normalization to generate an interference-plus-noise covariance matrix free of the desired signal and immune against model mismatch and steering vector mismatch [118].

\subsection{Array Signal Model and Adaptive Beamform- ing}

Adaptive beamforming is a technique used for combining the received signal of interest (SOI) from all receiving sensors from a specific direction while suppressing the interfering signals adaptively in other directions. The outputs of the individual sensors are linearly combined after being scaled with their corresponding weights. This technique can adaptively optimize the array pattern by adjusting the elemental control complex weights until a prescribed objective function is satisfied [119].

\subsubsection{Array Signal Model}

Consider a uniform linear array (ULA) of a collocated antenna system with $M$ signal sources impinging on $N$ receiving antennas. Let $\theta_{i}$ be the desired signal direction of arrival. The baseband received signal of the array at time instant $t$, $\mathbf{y}(t)=\left[y_{1}(t), y_{2}(t), \ldots, y_{N}(t)\right] \in \mathbb{C}^{M}$, can be represented as

$$
\mathbf{y}(t)=\mathbf{a}\left(\theta_{i}\right) s(t)+\mathbf{j}(t)+\mathbf{n}(t)
$$

where

$$
\mathbf{a}\left(\theta_{i}\right)=\left[1, \quad e^{-j 2 \pi \frac{d}{\lambda} \sin \left(\theta_{i}\right)}, \ldots, e^{-j 2 \pi(N-1) \frac{d}{\lambda} \sin \left(\theta_{i}\right)}\right]^{T}
$$

is the steering vector associated with $\theta_{i}, d$ is the distance between two consecutive antennas, $\lambda$ is the wavelength, $s(t)$ is the desired signal waveform, $\mathbf{j}(t)$ is the interference and $\mathbf{n}(k)$ is independent identically distributed additive Gaussian noise. For 
multiple received signals, the matrix form can be written as

$$
\mathbf{Y}=\mathbf{A}(\theta) \mathbf{S}+\mathbf{J}+\mathbf{N}
$$

where $\mathbf{A}=\left[\mathbf{a}\left(\theta_{1}\right), \mathbf{a}\left(\theta_{2}\right), \ldots, \mathbf{a}\left(\theta_{M}\right)\right]$ is the array manifold matrix, $\mathbf{S}=$ $\left[\mathbf{s}_{1}(t), \mathbf{s}_{2}(t), \ldots, \mathbf{s}_{M}(t)\right]$ are the received signals, $\mathbf{J}$ is the interference matrix and $\mathbf{N}$ is the noise matrix. The output of the beamformer for multiple received signals can expressed as

$$
\begin{aligned}
\boldsymbol{\omega}^{H} \mathbf{Y} & =\boldsymbol{\omega}^{H} \mathbf{S}+\boldsymbol{\omega}^{H} \mathbf{J}+\boldsymbol{\omega}^{H} \mathbf{N} \\
& =\boldsymbol{\omega}^{H} \mathbf{S}+\boldsymbol{\omega}^{H}\left(\mathbf{X}_{j+n}\right)
\end{aligned}
$$

where $\boldsymbol{\omega}=\left[\omega_{1}, \omega_{2}, \ldots, \omega_{N}\right]$ is the beamforming complex weights and $\mathbf{X}_{j+n}=\mathbf{J}+\mathbf{N}$ is the interference-plus-noise matrix. A typical adaptive beamforming block diagram is illustrated in Fig. 5.1.

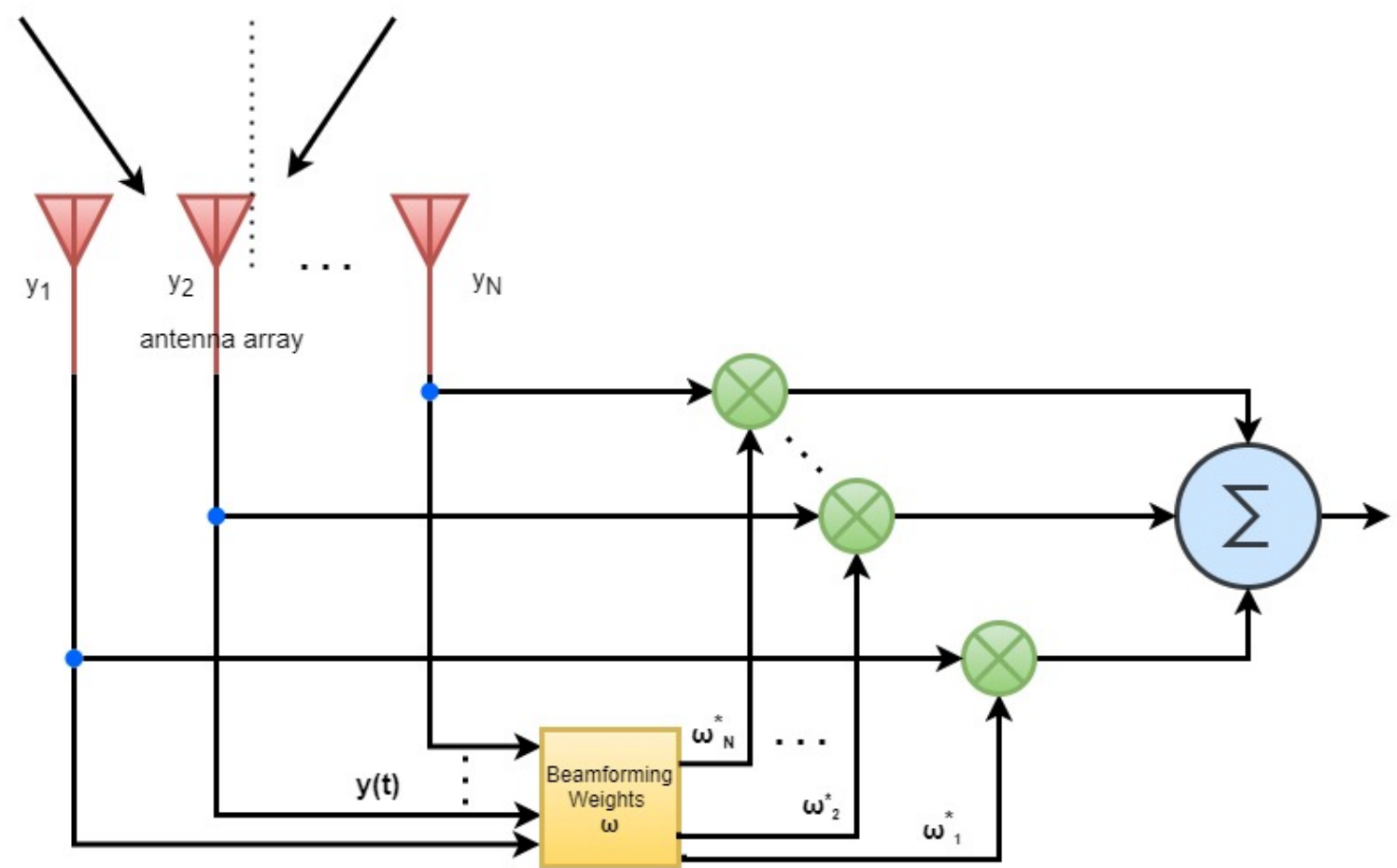

Figure 5.1: Block diagram of adaptive beamforming system. 


\subsubsection{MVDR Beamforming Algorithm}

The common goal of narrowband adaptive beamforming techniques is to maximize the output SNR. The maximization of SNR can be achieved by minimizing the total output power subject to the constraint that the desired signal is not distorted. This leads to the MVDR beamforming algorithm which adjusts the complex weight factors to minimize the interference and noise in the desired direction while maintaining a unity gain desired signal with minimal distortion [90]. Using (5.4), the power of the interference-plus-noise of the output can be express as

$$
\begin{array}{r}
\mathbb{E}\left\{\left|\boldsymbol{\omega}^{H} \mathbf{J}+\boldsymbol{\omega}^{H} \mathbf{N}\right|^{2}\right\}=\mathbb{E}\left\{\left|\boldsymbol{\omega}^{H} \mathbf{X}_{j+n}\right|^{2}\right\} \\
=\boldsymbol{\omega}^{H} \mathbb{E}\left\{\mathbf{X}_{j+n} \mathbf{X}_{j+n}^{H}\right\} \boldsymbol{\omega}=\boldsymbol{\omega}^{H} \mathbf{R}_{j+n} \boldsymbol{\omega}
\end{array}
$$

where $\mathbf{R}_{j+n}$ is the covariance matrix for the interference-plus-noise. Similarly, from (5.4), a necessary and sufficient condition is required to maximize the distortionless output in the desired direction, which can be expressed as

$$
\boldsymbol{\omega}^{H} \mathbf{a}=1
$$

where $\mathbf{a}=\mathbf{s}(\theta) / \sqrt{\mathbf{s}^{H}(\theta) \mathbf{s}(\theta)}$ is a normalized directional vector associated with the steering vector at angle $\theta$. Therefore, the MVDR beamforming algorithm can be written as the optimization problem

$$
\begin{array}{cc}
\min _{\boldsymbol{\omega}} & \boldsymbol{\omega}^{H} \mathbf{R}_{j+n} \boldsymbol{\omega} \\
\text { s.t. } & \boldsymbol{\omega}^{H} \mathbf{a}=1 .
\end{array}
$$

By applying the Lagrange multiplier $t,(5.7)$ can be changed into

$$
G(\boldsymbol{\omega})=\frac{1}{2} \boldsymbol{\omega}^{H} \mathbf{R}_{j+n} \boldsymbol{\omega}+t\left(\boldsymbol{\omega}^{H} \mathbf{a}-1\right)
$$

The optimal weight vector for the MVDR beamforming algorithm is expressed as [86]

$$
\boldsymbol{\omega}=\mathbf{R}_{j+n}^{-1}\left(\frac{\mathbf{a}}{\mathbf{a}^{H} \mathbf{R}_{j+n}^{-1} \mathbf{a}}\right)
$$




\subsection{Overview of Interference-plus-Noise Covari- ance Matrix Estimation Techniques}

The MVDR beamforming algorithm requires an accurate interference-plus-noise covariance matrix $\mathbf{R}_{j+n}$. Inadequate estimation of the covariance matrix results in degraded performance in the MVDR beamforming algorithm, which leads to antenna patterns with high sidelobes and distorted main-beams [114,120]. The sample covariance matrix suffers from large inaccuracy, especially when the number of snapshots are limited [121]. The diagonal loading technique can be used to correct the inaccuracy of the sample covariance matrix $\mathbf{R}_{s c m}$, which is defined as

$$
\mathbf{R}_{s c m}=\frac{1}{K} \sum_{k=1}^{K} \mathbf{y}(k) \mathbf{y}^{H}(k)
$$

where $K$ is the number of received snapshots. The diagonally loaded interferenceplus-noise covariance matrix $\widetilde{\mathbf{R}}_{j+n}^{-1}$ is derived to be

$$
\widetilde{\mathbf{R}}_{j+n}^{-1}=\left(\mathbf{R}_{s c m}+\iota \mathbf{I}\right)^{-1}\left\{\mathbf{I}-\frac{\varepsilon}{\iota+\sigma_{n}^{2}} \mathbf{B C}\right\}
$$

where

$$
\mathbf{C}=\left[\mathbf{I}-\mathbf{S}\left(\mathbf{S}^{H} \mathbf{S}+\left(\sigma_{n}^{2}+\iota\right) \Omega^{-1} \mathbf{S}^{-1}\right)\right]
$$

and where $\varepsilon=\operatorname{std}\left(\operatorname{diag}\left(\mathbf{R}_{s c m}\right)\right)$ is the inaccuracy of $\mathbf{R}_{s c m}$,std refers to the standard deviation, $\sigma_{n}$ is the noise power, $\mathbf{B}$ is a random matrix with zero mean and unit variance and all its elements are $\gg 1, \mathbf{S}$ is the array manifold matrix, $\Omega$ is a diagonal matrix associated with the interference signal power and $\iota$ is the diagonal loading bounded by $\varepsilon-\sigma_{n}^{2} \leqslant \iota \ll \mathbf{R}_{s c m}(i, i)$, where $\mathbf{R}_{s c m}(i, i)$ are the diagonal elements of the sample covariance matrix. For the complete derivation, see Appendix B.1.

The diagonally loaded sample covariance is a popular estimation technique for estimating unknown covariance matrices. However, while it is an unbiased estimator, it does not minimize the mean-squared error (MSE). On the other hand, the shrinkage algorithm can achieve better performance in comparison with the diagonal loading technique especially for small sample size $n$ and larger dimension $p$ [122]. Ledoit and Wolf (LW) [115] proposed a shrinkage estimator which asymptotically minimizes the 
MSE. The LW estimator is well conditioned for small sample sizes and can thus be applied to high dimensional problems. The LW estimator can be defined as

$$
\widehat{\mathbf{R}}_{L W}=\left(1-\hat{\rho}_{L W}\right) \mathbf{R}_{S C M}+\hat{\rho}_{L W} \widehat{\mathbf{F}}
$$

where

$$
\hat{\rho}_{L W}=\frac{\sum_{i=1}^{n}\left\|\mathbf{x}_{i} \mathbf{x}_{i}^{T}-\mathbf{R}_{S C M}\right\|_{F}^{2}}{n^{2}\left[\operatorname{tr}\left(\mathbf{R}_{S C M}^{2}\right)-\operatorname{tr}^{2}\left(\mathbf{R}_{S C M}\right) / p\right]}
$$

is the shrinkage coefficients, $\widehat{\mathbf{F}}$ is the shrinkage target, $\mathbf{x}_{i}$ is the data sample, $n$ is the number of snapshots, $p$ is the data dimension, $\operatorname{tr}$ is the trace of the matrix and $\|\cdot\|_{F}$ is the Frobenius norm. More details are provided in Appendix B.2.

The eigenspace decomposition of the sample covariance matrix technique is another major approach for robust adaptive MVDR beamforming. The main idea of this technique is to separate the interference subspace from the signal subspace by using Capon's spatial spectrum [90]. The desired signal eigenvector obtained from Capon's spatial spectrum is used to reconstruct the signal covariance matrix and subtract from the sample covariance matrix to acquire the interference-plus-noise covariance matrix [116]. The interference-plus-noise covariance matrix can be written as

$$
\widehat{\mathbf{R}}_{j+n}=\mathbf{R}_{S C M}-\hat{\lambda}_{z} \hat{\mathbf{v}}_{z} \hat{\mathbf{v}}_{z}^{H}
$$

where $\hat{\lambda}_{z}$ is the eigenvalue of the sample covariance matrix corresponding to the desired signal waveform $z(k)$ impinging from a specific angular sector and $\hat{\mathbf{v}}_{z}$ can be estimated using

$$
\hat{\mathbf{v}}_{z}=\underset{\mathbf{v} \in \mathbf{V}}{\operatorname{argmax}} \frac{\left\|\mathbf{V}_{z} \mathbf{V}_{z}^{H} \mathbf{v}\right\|}{\left\|\mathbf{I}-\mathbf{V}_{z} \mathbf{V}_{z}^{H} \mathbf{v}\right\|}
$$

where $\mathbf{V}_{z}$ is the column orthogonal matrix of the columns of the reconstructed desired signal covariance matrix. More details can be found in Appendix B.3.

Another important technique based on sparsity is used to estimate the interference-plus-noise covariance matrix free of desired signal. The key idea of the sparsity-based interference-plus-noise covariance matrix estimation is to exploit the sparsity of sources in the observed spatial domain and reconstruct the interferenceplus-noise covariance as a linear combination of the outer products of the interference 
steering vectors weighted by their individual power [117]. In comparison with the classical methods, the sparsity-constrained covariance matrix can be formulated as a convex optimization problem by relaxing the $\ell_{0}$-norm and approximately replacing it by an $\ell_{1}$-norm. This convex optimization problem can be reformulated as a basis pursuit de-noising problem [123] as

$$
\begin{aligned}
\min _{\boldsymbol{p}, \sigma_{n}^{2}} & \left\|\mathbf{R}_{S C M}-\mathbf{S P S}^{H}-\sigma_{n}^{2} \mathbf{I}\right\|_{F}+\varsigma\|\mathbf{p}\|_{1} \\
\text { s.t. } & \mathbf{p} \geqslant 0
\end{aligned}
$$

where $\mathbf{p} \in \mathbb{R}_{+}^{M}$ is the spatial spectrum distribution on the sample grid of the observed spatial domain $\left(e . g .,\left\{\theta_{1}, \theta_{2}, \ldots, \theta_{M}\right\} \in \Theta\right), \mathbf{P}=\operatorname{diag}(\mathbf{p})$ is the corresponding diagonal matrix, $\mathbf{S}$ is the array manifold matrix, $\sigma_{n}^{2}$ is the noise power, $\varsigma$ is a regularization parameter controlling the trade-off between the sparsity of the spatial spectrum and the residual norm of covariance matrix and $\|\cdot\|_{0}$ is the $\ell_{0}$-norm of a vector. The optimization problem is convex and can be solved using standard and highly efficient interior point methods. In comparison with the previously discussed techniques, the sparsity-based interference-plus-noise covariance matrix estimation does not require matrix inversion or eigen-decomposition. However, the solution of this optimization problem is not absolutely sparse because of the $\ell_{1}$-norm relaxation [117]. For more details see Appendix B.4.

\subsection{Interference-plus-Noise Covariance Matrix Es- timation in Riemannian Space}

The previous sections illustrated the state of the art in interference-plus-noise covariance matrix estimation. The sample covariance matrix is a maximum likelihood estimate of the data covariance matrix, which leads to an optimal adaptive beamformer when the sample size tends to infinity. However, the sample size is often limited, thus resulting in substantial performance degradation $[113,124]$. The most popular interference-plus-noise technique is the diagonal loading technique [121], which adds a scaled identity matrix to the sample covariance matrix to reduce the conditional number. However, the diagonal loaded sample covariance matrix is not free of target, which leads to degradation in performance under small sample size. The shrinkage estimation method [115] minimizes the mean square error and can compute the diagonal 
loading levels from the data sample. However, this technique estimates the statistical covariance matrix of the array received data rather than the required interferenceplus-noise covariance matrix. In such a case, the performance degradation becomes severe with the increase of the desired signal power. The eigenspace decomposition technique [125] is another popular method for estimating the interference-plus-noise covariance matrix. The main idea of this technique is to separate the interference subspace from the signal space by exploiting Capon's spatial spectrum. It is known that this approach suffers severe performance degradation under low SNR [116]. The sparsity based interference-plus-noise covariance matrix estimation technique exploits the $\ell_{1}$-norm to formulate a convex optimization. This optimization requires extra computations to converge to a solution and the regularization parameter can be difficult to determine in some situations [117].

The interference-plus-noise covariance matrix estimation can be viewed from the Riemannian geometry viewpoint where every snapshot is converted to a THPD covariance matrix that resides on the Riemannian manifold. These points on the Riemannian manifold can be manipulated using the Riemannian mean and the Riemannian distance. This section proposes a new technique to tackle the challenge of estimating the interference-plus-noise covariance matrix to improve the MVDR optimal beamforming.

The Riemannian geometry-based interference-plus-noise covariance matrix technique is formulated as a linear combination of all THPD covariance matrices residing on the Riemannian manifold. The weights of the linear combination can be determined from distance between the Riemannian mean and all the THPD covariance matrices. Using the max-min normalization, the weights for the further THPD points from the Riemannian mean are minimum and the weights for closer THPD points from the Riemannian mean are maximum. The largest Riemannian distance will have zero weight and the smallest Riemannian distance will have a weight of 1 .

\subsubsection{Signal Model}

Consider a ULA of collocated antenna system with $M$ sources impinging on an $N$ receiving antennas. Let $\theta_{i}$ be the desired signal direction of arrival. The received 
signals for the $k^{\text {th }}$ snapshot can be modelled as

$$
\mathbf{y}[k]=\sum_{i=1}^{M} \mathbf{a}\left(\theta_{i}\right) s_{i}[k]+\mathbf{j}[k]+\mathbf{n}[k]
$$

where $\mathbf{s}[k]=\left(s_{1}[k], \ldots, s_{M}[k]\right)$ is the received signal, $\mathbf{n}[k]$ is independent identically distributed additive Gaussian noise, $\mathbf{j}[k]$ is the interference from other sources and $\mathbf{A}=\left[\mathbf{a}\left(\theta_{1}\right), \ldots, \mathbf{a}\left(\theta_{M}\right)\right]$ is the array manifold matrix. The standard model for interference-plus-noise can be defined as [126]

$$
\mathbf{d}=\sum_{q=1}^{Q} \mathbf{a}\left(\theta_{q}\right) \mathbf{b}_{q}+\mathbf{n}
$$

where $Q$ is the number of interferers, $\theta_{q}$ is the interferer's angle of arrival and the $\mathbf{b}_{q}$ are the complex amplitudes of the interferers. Consequently, the asymptotic covariance matrix of the interference plus noise can be written

$$
\begin{aligned}
\mathbf{R}_{j+n} & =\mathbb{E}\left[\mathbf{d d}^{H}\right] \\
& =\mathbf{A}_{J} \mathbf{B} \mathbf{A}_{J}^{H}+\beta^{2} \mathbf{I}
\end{aligned}
$$

where $\mathbf{B}=\mathbb{E}\left[\mathbf{b b}^{H}\right], \mathbf{A}_{J}=\left[\mathbf{a}\left(\theta_{1}\right), \ldots, \mathbf{a}\left(\theta_{Q}\right)\right], \beta$ is the noise power and $\mathbf{I}$ is the identity matrix.

\subsubsection{Riemannian Geometry-based Interference-plus-Noise Covariance Matrix Estimation}

Consider $K$ received radar snapshots, where every snapshot can be converted to a THPD covariance matrix using the RBA algorithm in Sec. 2.4. The first step in estimating the interference-plus-noise covariance matrix $\tilde{\mathbf{R}}_{j+n}$ is determining the Riemannian mean $\mathbf{R}_{r m}$ of all the THPD covariance matrices points $\left(\mathbf{R}_{1}, \ldots, \mathbf{R}_{K}\right)$ on the Riemannian manifold using (2.21). Then, using (2.18) to determine the Riemannian distances between the Riemannian mean and all points on the Riemannian manifold,

$$
\mathbf{d}_{\mathbf{R}}=\left[d_{\mathrm{R}}\left(\mathbf{R}_{r m}, \mathbf{R}_{1}\right), \ldots, d_{\mathrm{R}}\left(\mathbf{R}_{r m}, \mathbf{R}_{K}\right)\right]
$$


where $\mathbf{d}_{\mathbf{R}}$ is a vector of Riemannian distances. According to [51], the Riemannian distance-based constant false alarm rate detector calculates the Riemannian distance between the Riemannian mean of the reference HPD covariance matrices and the HPD covariance matrix under test. The larger the Riemannian distance, the higher the probability of the HPD covariance matrix under test being a potential target. The estimated interference-plus-noise covariance matrix has to be free of targets, hence the next step is to remove the THPD covariance matrix associated with the largest

distance. The estimated $\tilde{\mathbf{R}}_{j+n}$ is formulated as a linear combination of the remaining THPD covariance matrices. The weights are based on the max-min normalization [127] of the Riemannian distance as

$$
\alpha_{k}=\frac{d_{\mathrm{R}}\left(\mathbf{R}_{r m}, \mathbf{R}_{k}\right)-\max \left(\mathbf{d}_{\mathbf{R}}\right)}{\min \left(\mathbf{d}_{\mathbf{R}}-\max \left(\mathbf{d}_{\mathbf{R}}\right)\right)} .
$$

The largest Riemannian distance, which is the farthest point from the Riemannian mean $\mathbf{R}_{r m}$, will have zero weight and the smallest distance which is the closest to the Riemannian mean will, have a weight of 1 . The estimated $\tilde{\mathbf{R}}_{j+n}$ can be defined as [118]

$$
\tilde{\mathbf{R}}_{j+n}=\alpha_{1} \mathbf{R}_{1}+\cdots+\alpha_{K} \mathbf{R}_{K} .
$$

This estimated $\tilde{\mathbf{R}}_{j+n}$ puts more weight on the points that are close to the mean and less weight on the points that are farther out from the mean.

\subsection{Simulation Results}

The simulation results measure the performance of the MVDR beamformer with diagonally loaded, shrinkage, subspace, sparse and the Riemannian geometry interferenceplus-noise covariance matrices. The simulations are based on a collocated uniform linear array with $N=8$ receivers and the antenna elements spaced half a wavelength apart from each other. The desired direction of arrival is $\theta_{i}=30$ degrees and two sources of interference are considered with directions of arrival $\{0,50\}$ degrees. The input signal to interference-plus-noise ratio varies from $-10 \mathrm{~dB}$ to $10 \mathrm{~dB}$ with different sample sizes. The main task in this simulation is to measure the performance of all techniques under different sample sizes at high and low SINR. The diagonal loading factor is calculated with every new input data. The solution for 
the sparse-based interference-plus-noise covariance optimization problem is obtained using CVX [128] in Matlab. In addition, the Riemannian geometry-based interferenceplus-noise covariance matrix estimation exploits RBA with regularization parameter set to $\gamma_{1}=0.01$ and the autoregressive order model is $N=8$. Also, the Riemannian mean step size is $\epsilon=0.01$ and the number of iterations is set to $t=1000$ with tolerance level set to $10^{-5}$.

The first part of this simulation emphasises on comparing the estimated interference-plus-noise covariance matrices for all presented techniques with the asymptotic interference-plus-noise covariance matrix. One way to compare the asymptotic covariance matrix with another covariance matrix is to use the Siegel distance in (2.19), which is the geodesical distance between two covariance matrices. Figure 5.2 shows the Siegel distance between the asymptotic interference-plus-noise covariance and diagonally loaded, shrinkage, subspace, sparse and Riemannian geometry interference-plus-noise covariance matrices. Using Siegel distance between the asymptotic interference plus noise covariance and the Riemannian geometry-based interference-plus-noise covariance matrix converges faster as we increase the number of snapshots in comparison with the other techniques where they require more snapshots to converge. This result indicates that the Riemannian geometry-based interference-plus-noise covariance matrix is very close to the asymptotic covariance matrix.

The second part of this simulation focuses on the MVDR beamforming performance of the presented techniques for large and small samples sizes and high and low SINR. Figures 5.3 to 5.5 show the beamforming performance for diagonally loaded, shrinkage, subspace, sparse and Riemannian geometry interference-plus-noise covariance matrices at different sample sizes and different SINR. At SNR of $10 \mathrm{~dB}$ and $\mathrm{K}=64$ snapshots, all techniques performed very well in terms of main-lobe at desired angle of arrival and notches at the interferences' angles. However, reducing the number of snapshots to $K=8$, both the shrinkage and diagonal loaded have poor performance in comparison with the other techniques. At SNR of $-10 \mathrm{~dB}$ and angle mismatch of 28 degrees (instead of 30 degrees), the Riemannian geometry-based interference-plusnoise covariance matrix estimation technique has superior performance in comparison with all other techniques. We notice the diagonal loading technique suffered from self-nulling. The shrinkage, subspace and sparse techniques have a degraded performance while the Riemannian geometry based techniques maintained a low sidelobe 
and showed immunity against steering vector mismatch.

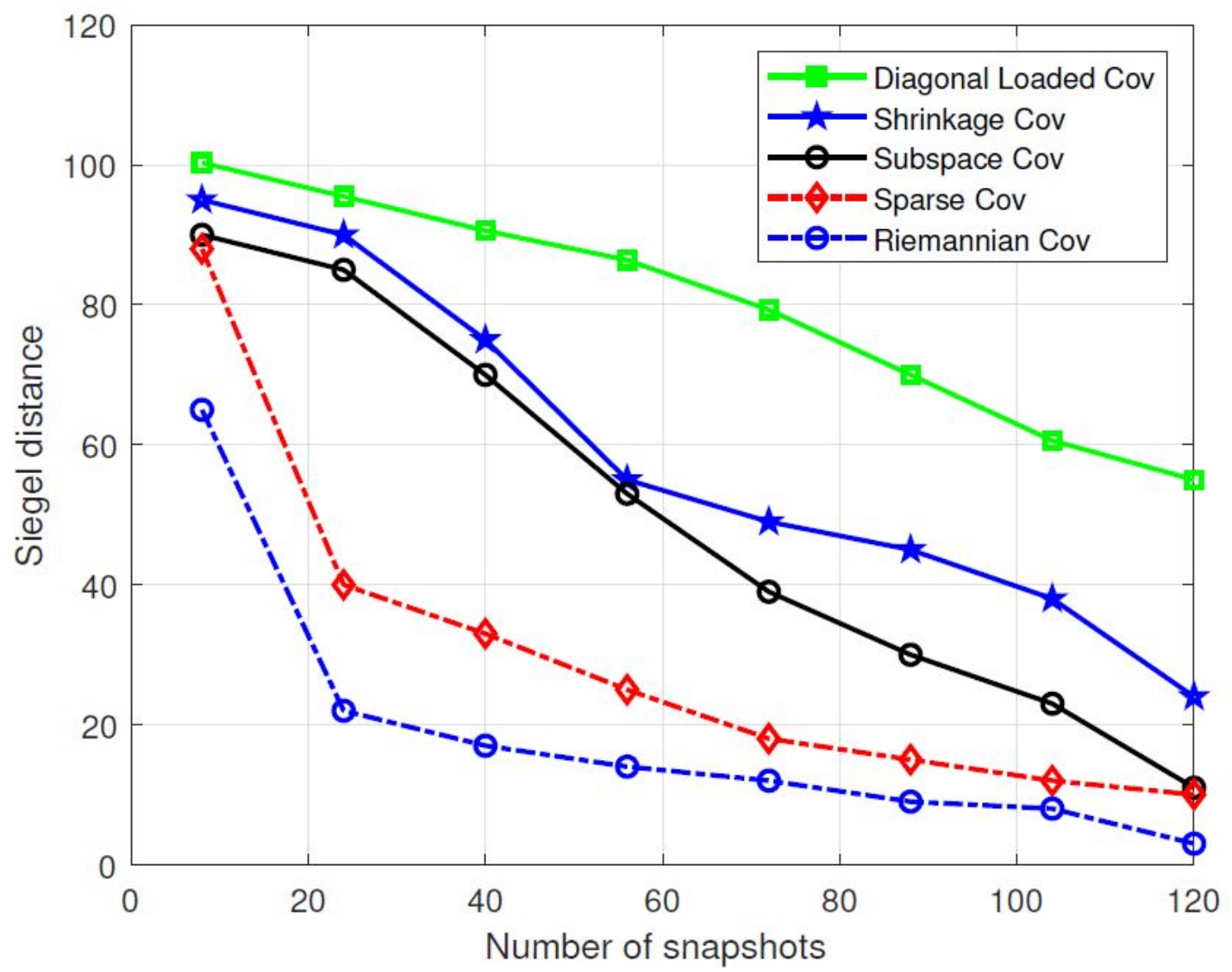

Figure 5.2: Siegel distance between the asymptotic interference-plus-noise covariance and diagonally loaded, shrinkage, subspace, sparse and Riemannian geometry interference-plus-noise covariance matrices. 


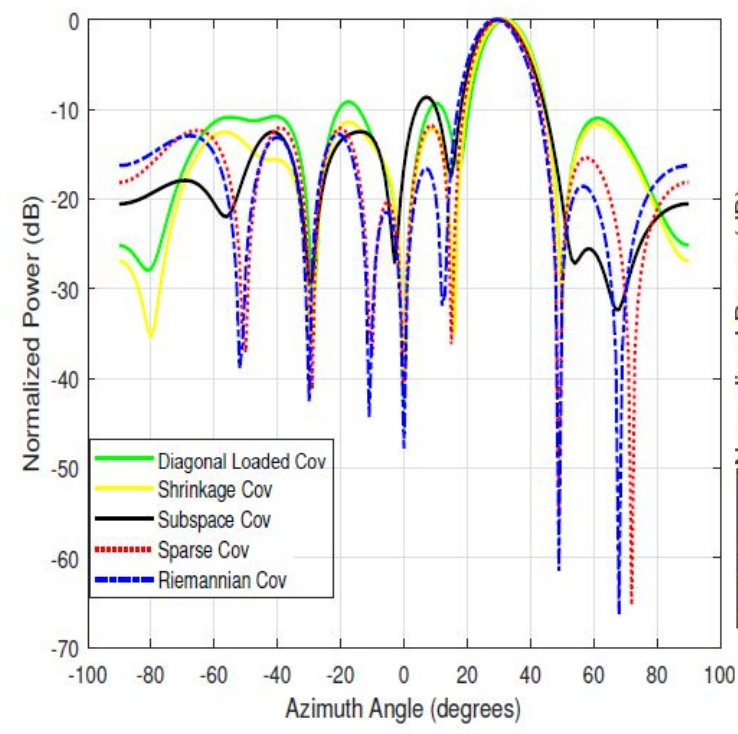

(a) $K=64$

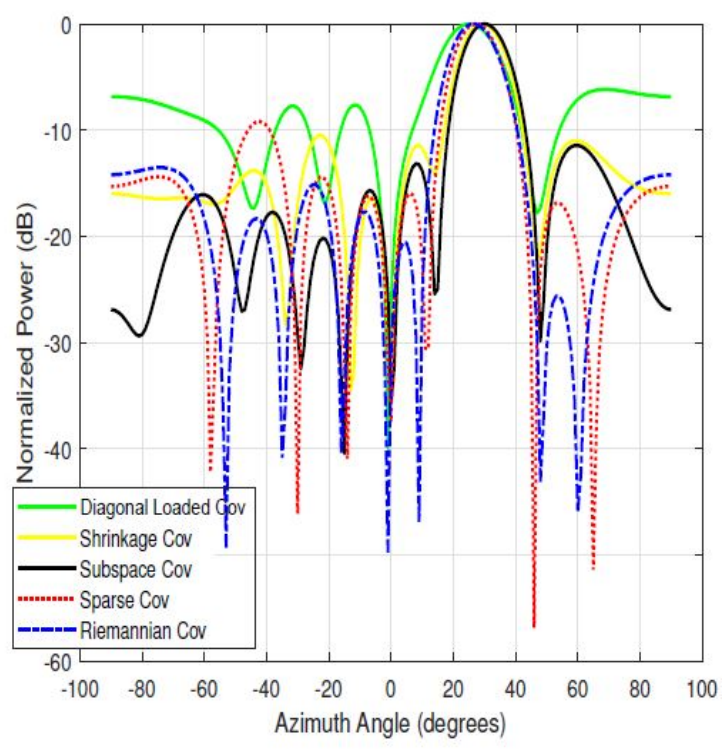

(c) $K=16$

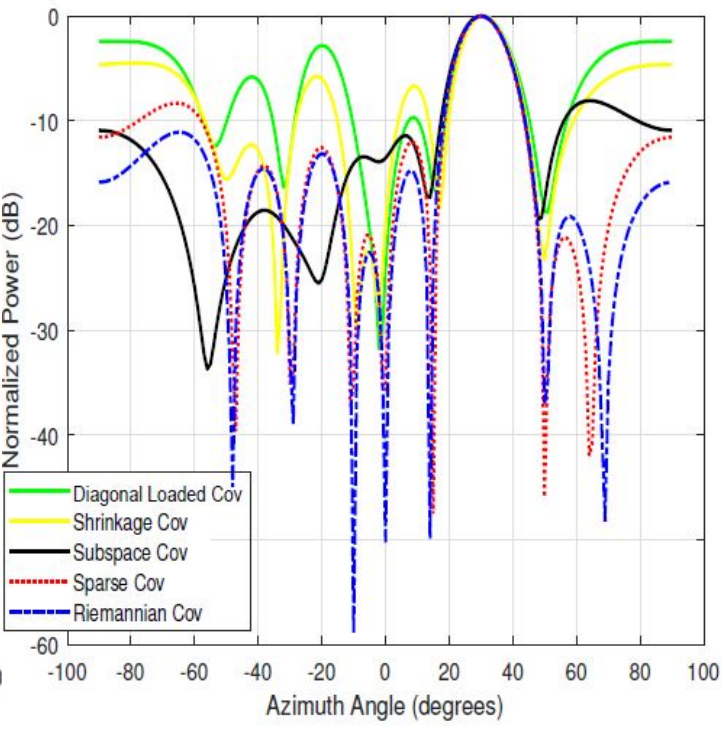

(b) $K=32$

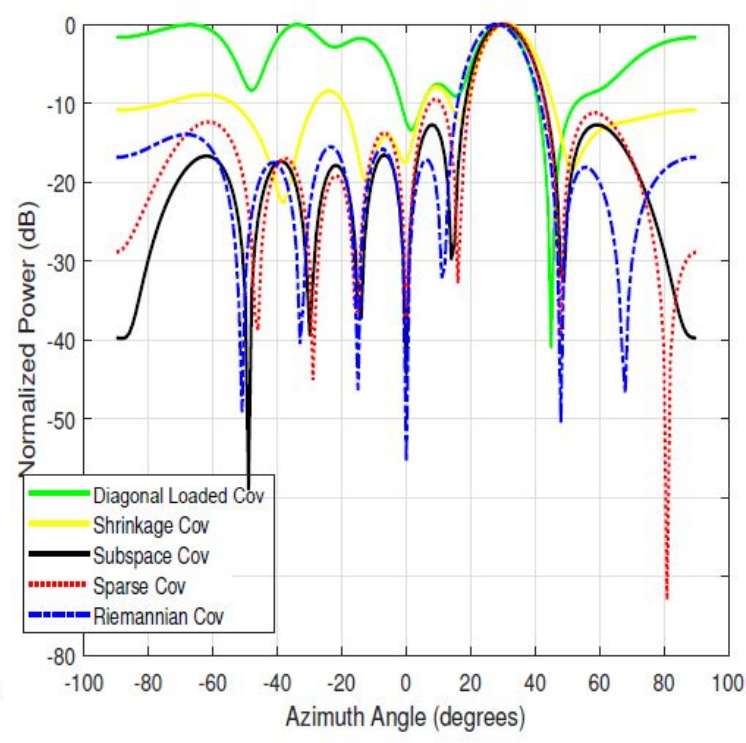

(d) $K=8$

Figure 5.3: MVDR beamforming for different interference-plus-noise covariance matrix estimation techniques at $\mathrm{SNR}=10 \mathrm{~dB}$. 


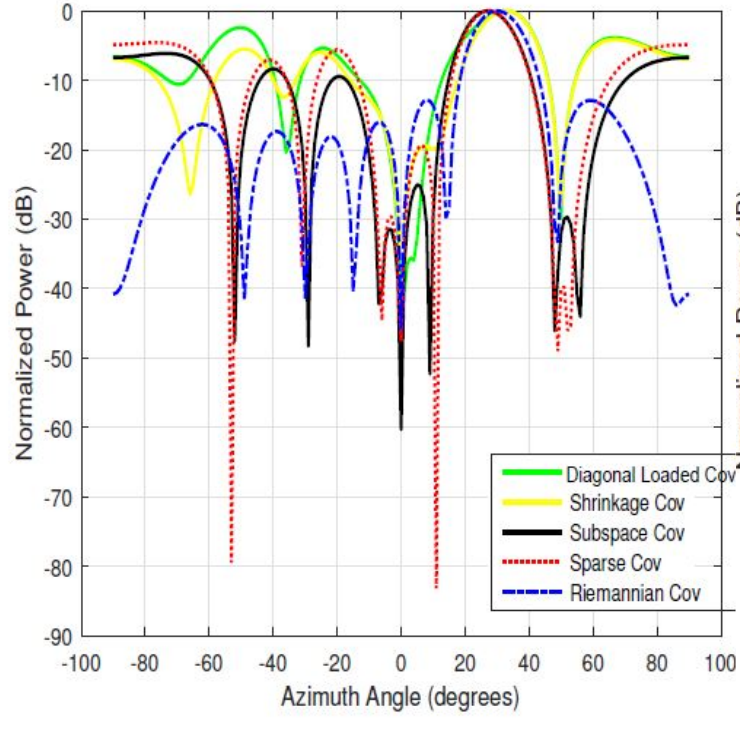

(a) $K=64$

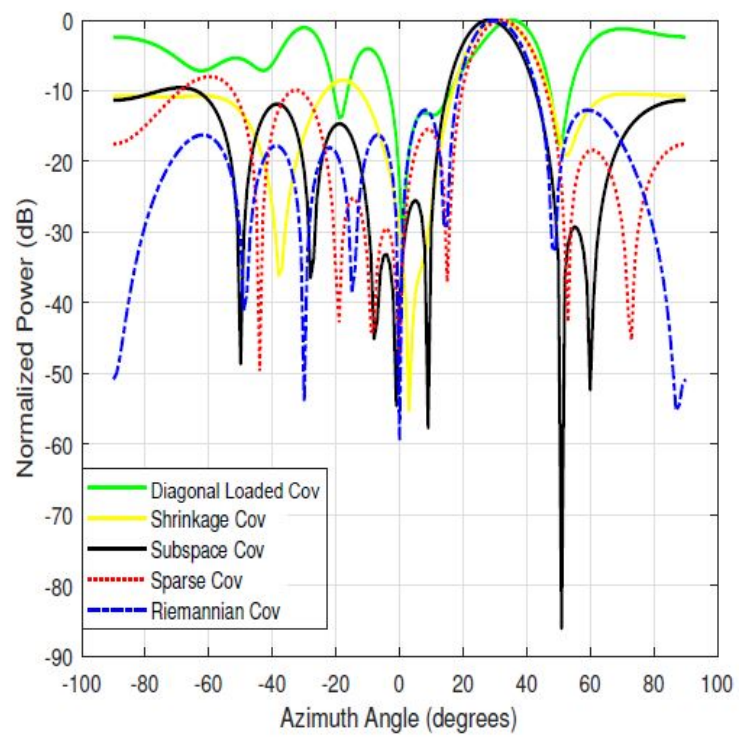

(c) $K=16$

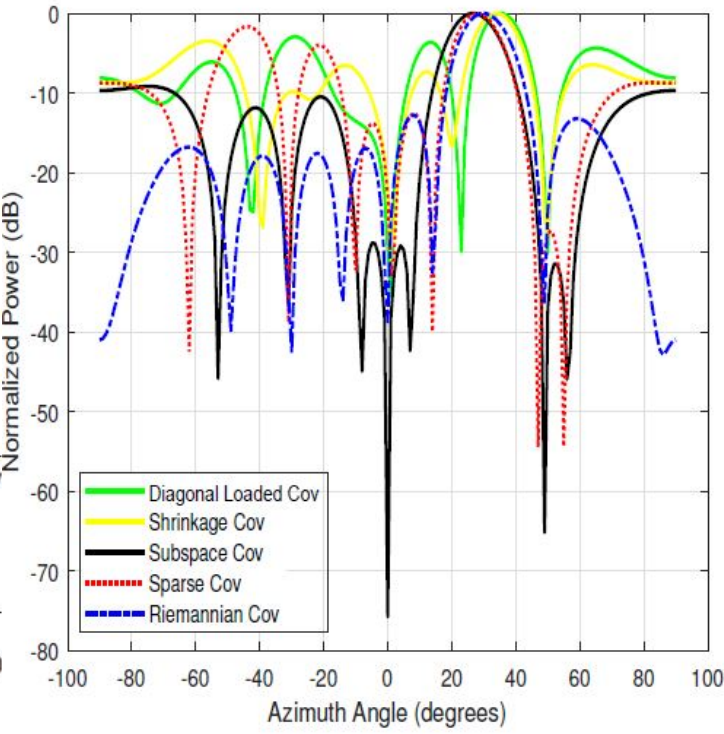

(b) $K=32$

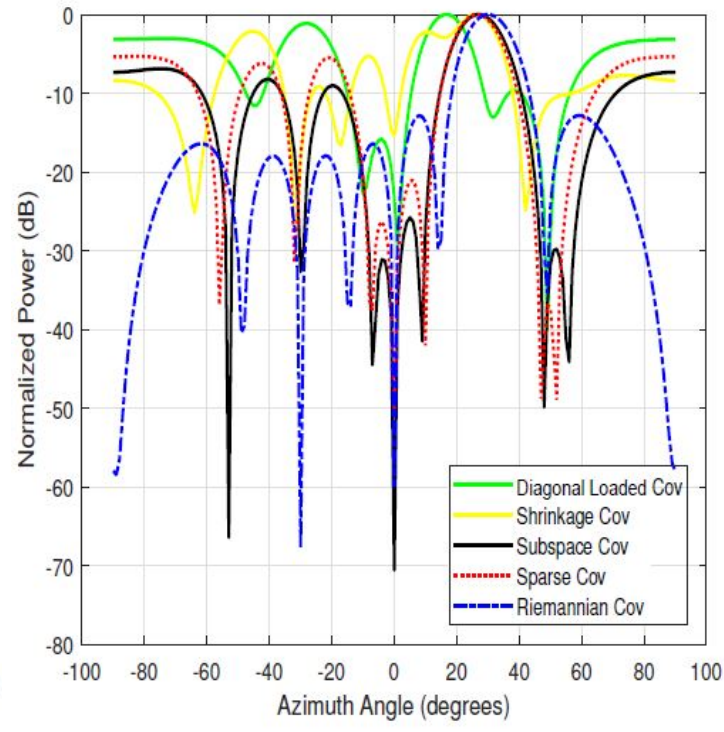

(d) $K=8$

Figure 5.4: MVDR beamforming for different interference-plus-noise covariance matrix estimation techniques at $\mathrm{SNR}=0 \mathrm{~dB}$. 


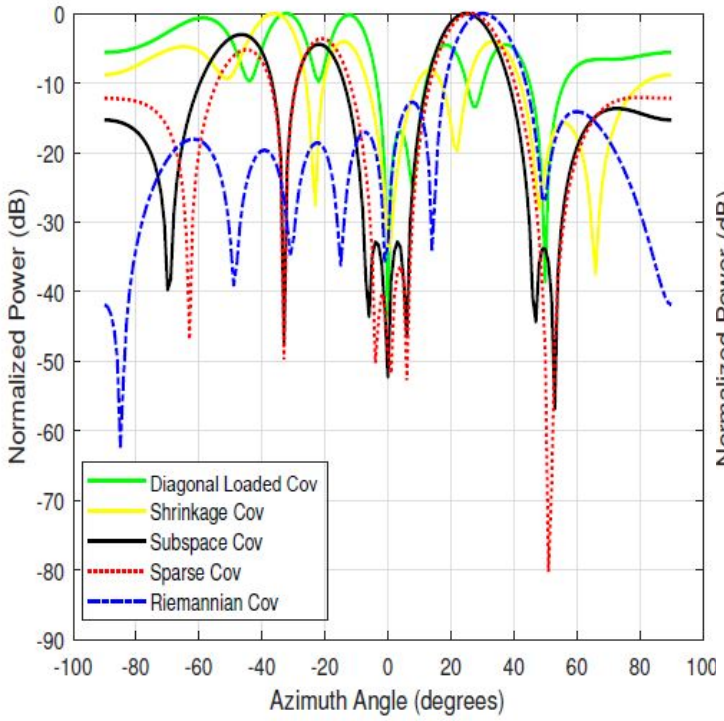

(a) $K=64$

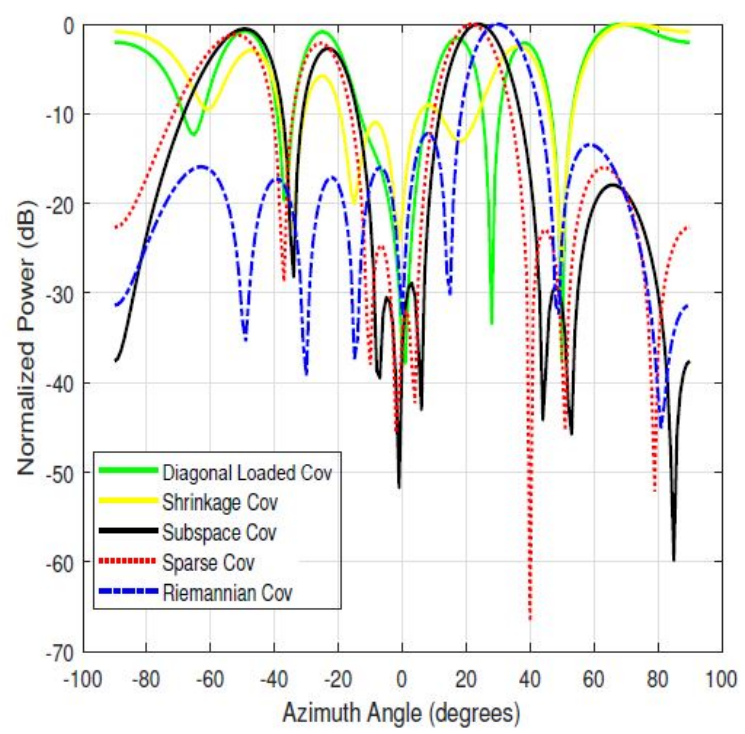

(c) $K=16$

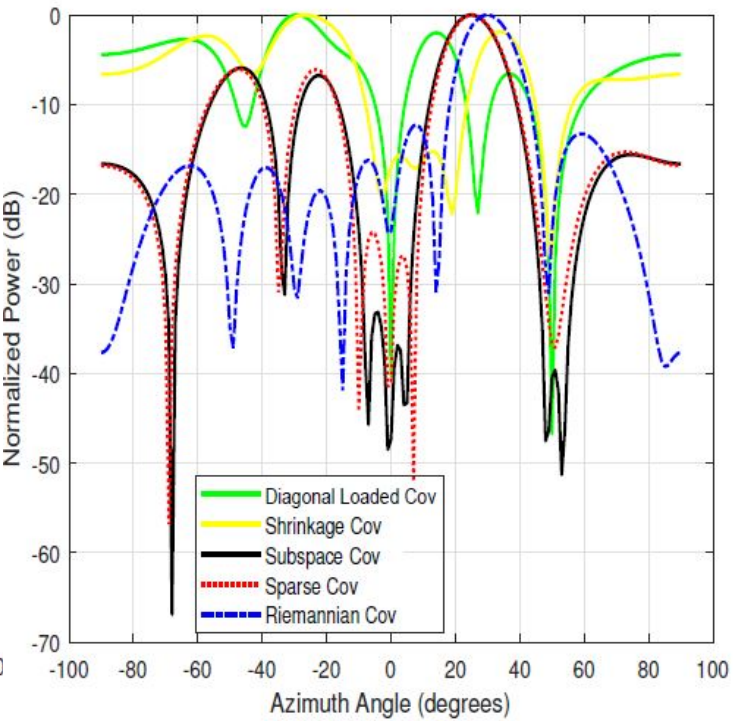

(b) $K=32$

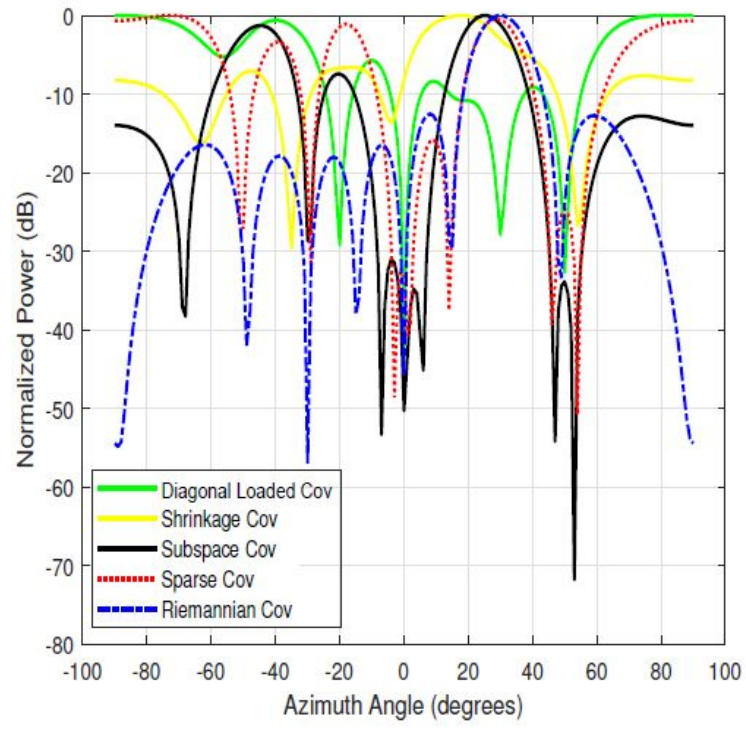

(d) $K=8$

Figure 5.5: MVDR beamforming for different interference-plus-noise covariance matrix estimation techniques at $\mathrm{SNR}=-10 \mathrm{~dB}$ with mismatch $\theta_{i}=28$ degrees. 


\subsection{Real Data Analysis}

The MVDR beamforming performance is tested on real data from a drone experiment. The data was collected using a Texas Instruments AWR1642 automotive radar with 28 degrees beam-width and a moving Yuneec Typhoon H drone target. The AWR1642 specification is listed in Table 3.1. The direction of arrival is estimated in Sec. 4.5.

The performance of the MVDR beamforming is examined on $K=8,16,32$ and 64 snapshots extracted from the original data set. The extracted snapshots are converted to THPD covariance matrices using RBA in Sec. 2.4 with regularization parameter set to $\gamma_{1}=0.1$ and the autoregressive order model is $N=4$. Also, the Riemannian mean step size is $\epsilon=0.01$ and the number of iterations is set to $t=1000$ with tolerance level set to $10^{-5}$. The MVDR beamforming for the Riemannian geometry-based interference-plus-noise covariance matrix is compared to diagonally loaded, shrinkage, subspace and sparse interference-plus-noise covariance matrices. Figure 5.6 shows the performance of the Riemannian, diagonally loaded, shrinkage, subspace and sparse interference-plus-noise covariance matrices. We notice that the performance of the diagonally loaded, shrinkage, subspace and sparse interference-plus-noise covariance matrices degrade as we decrease the number of snapshots. On the other hand, the Riemannian interference-plus-noise covariance matrix maintained the mainlobe at the estimated direction of arrival while keeping the sidelobes at $-10 \mathrm{~dB}$.

As an additional experiment, the SNR of the original data is scaled by 0.2 to examine the beamforming performance of the Riemannian interference-plus-noise covariance matrix in comparison with the other techniques. Figure 5.7 illustrates the MVDR beamforming performance of the Riemannian, diagonally loaded, shrinkage, subspace and sparse interference-plus-noise covariance matrices. The Riemannian interference-plus-noise covariance matrix maintained a robust performance as we decrease the number of snapshots with $-10 \mathrm{~dB}$ sidelobe levels. In addition, it conserved the mainlobe at the estimated direction of arrival. On the other hand, the other techniques suffered from severe degradation as we decrease the number of snapshots and the mainlobes diverted from the estimated direction of arrival. Hence, the MVDR beamforming under the Riemannian interference-plus-noise covariance matrix outperformed the other techniques for low SNR and small sample size. 


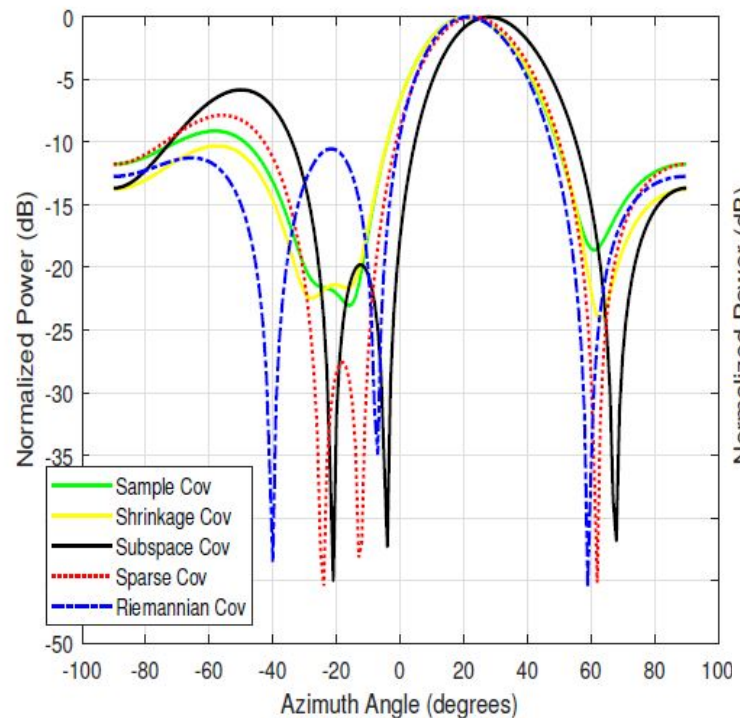

(a) $K=64$

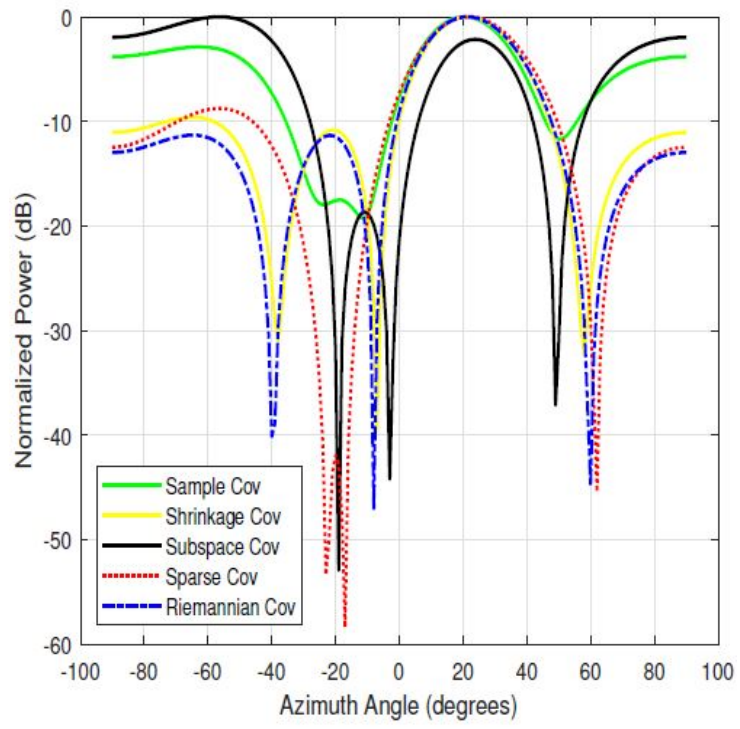

(c) $K=16$

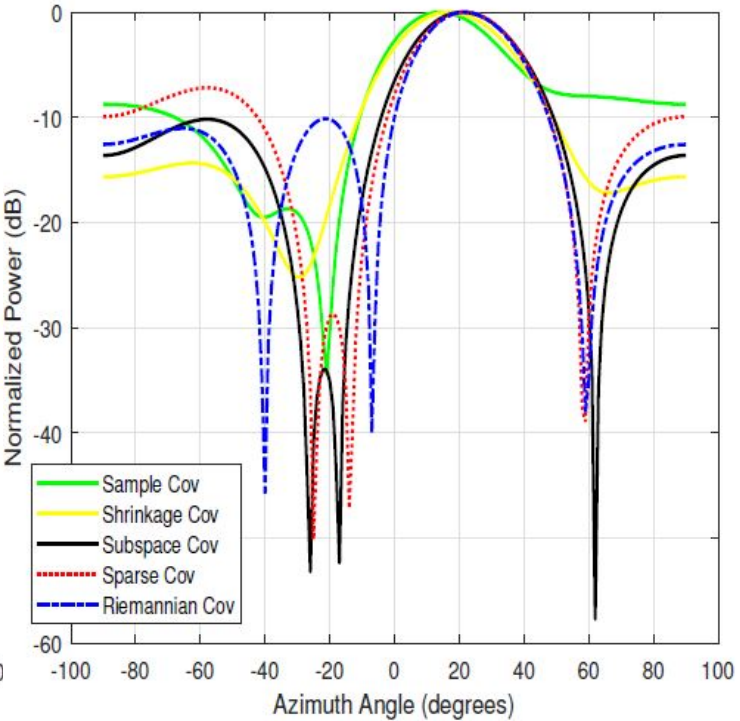

(b) $K=32$

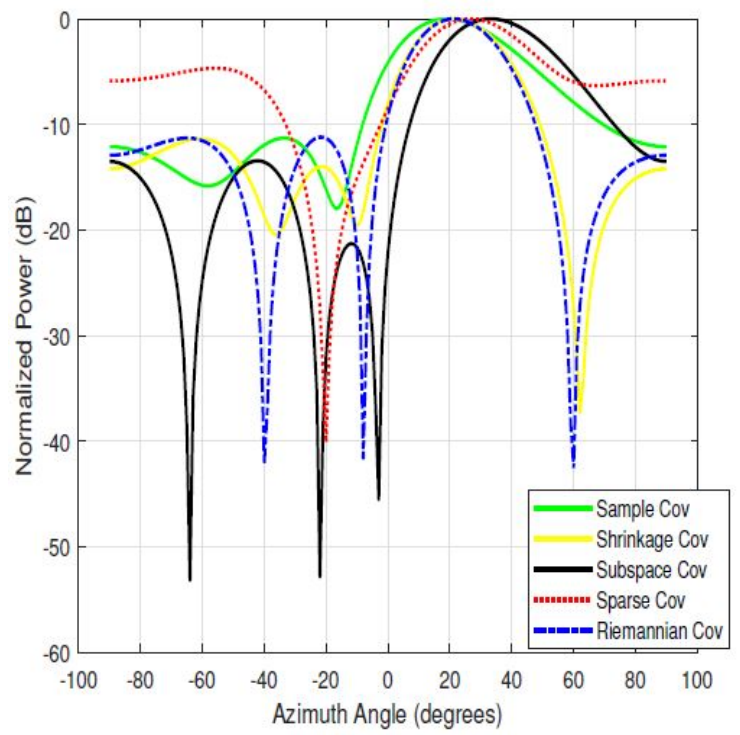

(d) $K=8$

Figure 5.6: MVDR beamforming for different interference-plus-noise covariance matrix estimation techniques for original data. 


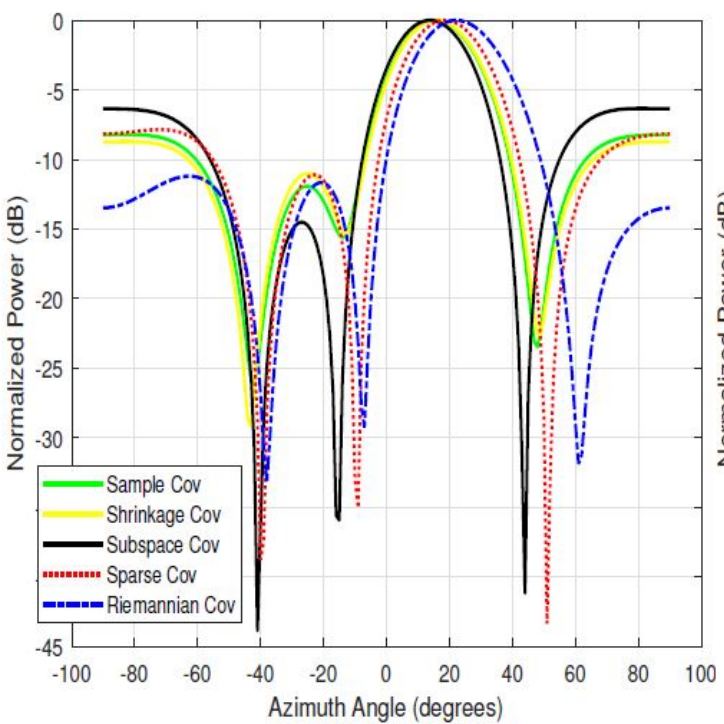

(a) $K=64$

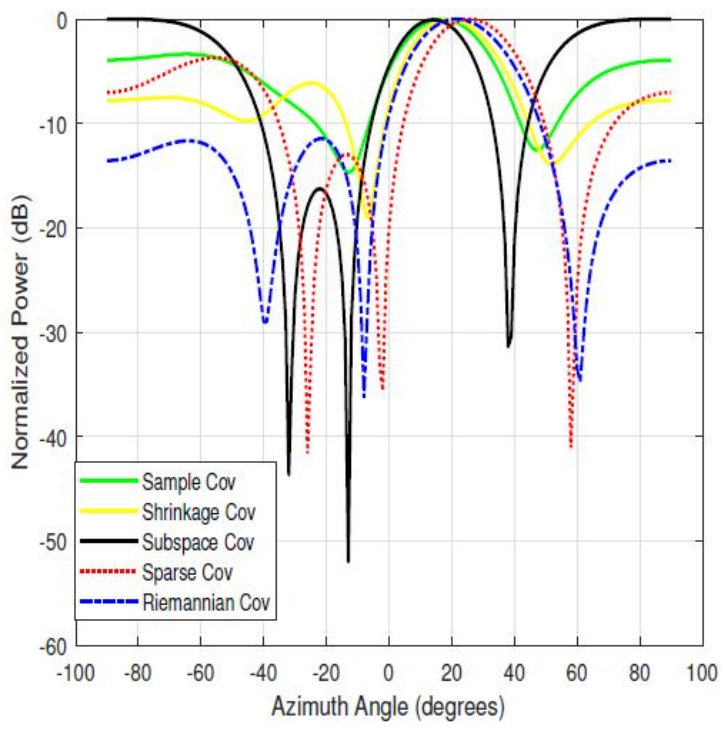

(c) $K=16$

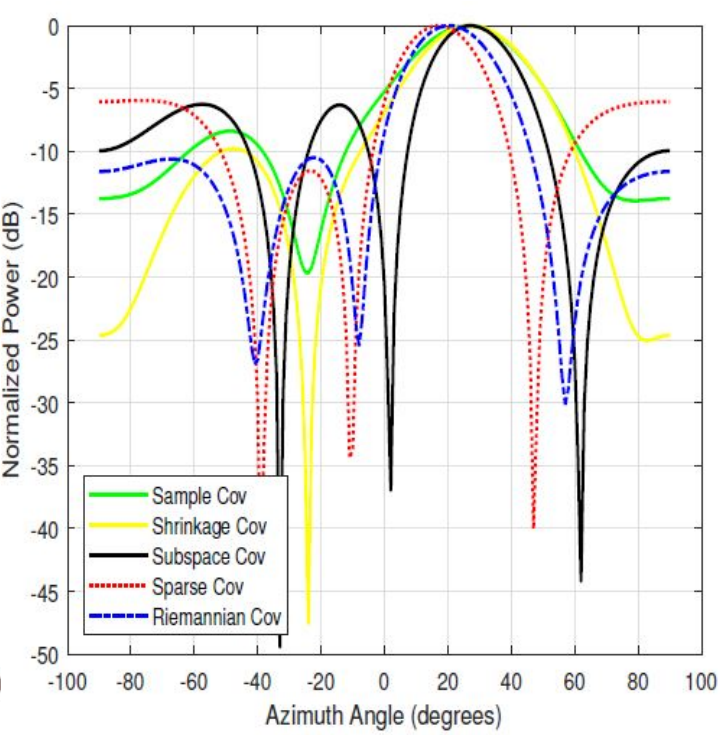

(b) $K=32$

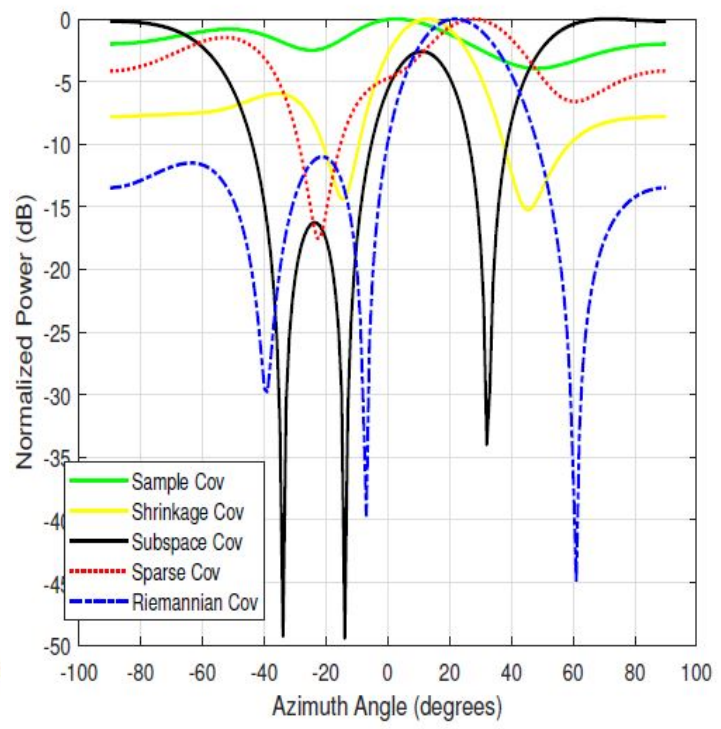

(d) $K=8$

Figure 5.7: MVDR beamforming for different interference-plus-noise covariance matrix estimation techniques for scaled SNR. 


\subsection{Conclusion}

In this chapter, the Riemannian geometry based interference plus noise covariance matrix estimation technique showed a superiority over the diagonally loaded, shrinkage, subspace and sparse based estimation techniques. Specifically, the interferenceplus-noise estimation problem is viewed from the Riemannian space perspective where every snapshot represent a point on the Riemannian manifold and can be manipulated using Riemannian metrics to generate an accurate interference plus noise covariance matrix with immunity against model mismatch and steering vector mismatch. Simulation results and real data analysis evidently demonstrate the effectiveness of the Riemannian geometry based technique under small number of snapshots and low SNR. 


\section{Chapter 6}

\section{Conclusion and Future Work}

\subsection{Conclusion}

Drones and micro-drones can be potential threats to public safety and military facilities. Thus, reliable drone detection and estimation techniques are much needed to allow countermeasures. The classical signal processing techniques may fail in presence of low radar cross section drones and limited number of received snapshots. The detection and estimation problems are projected into Riemannian space where the signal processing challenges can be mitigated.

In this dissertation, two Riemannian geometry-based CFAR detectors were developed to increase the probability of drone detection. The Riemannian-Brauer matrix $(\mathrm{RBM})$ and the angle-based hybrid Brauer (ABHB) CFAR detectors were presented as two stage approaches, exploiting the Brauer disc theorem, the Riemannian and Euclidean spaces. The two novel CFAR detectors have shown a superior performance in comparison with other CFAR detection techniques with both simulated and real data. Even with reduced SNR and limited the sample size, both the RBM and ABHB CFAR detectors maintained at least 2-5 dB gain over the other techniques while maintaining a low probability of false alarm under the uniform linear array (ULA) configuration. For non-ULA with random spacing between antenna elements and a slightly perturbed ULA configurations, the RBM and ABHB CFAR detectors suffered a loss of 1-2.5 dB when compared to the performance obtained with the ULA configuration.

Direction of arrival (DOA) estimation in the Riemannian space exploits the information geometry of each radar snapshot to enhance the DOA estimation. The DOA 
estimation problem in the Riemannian space is formulated as a linear search optimization model seeking the minimum Riemannian distance. The robust performance of the Riemannian space-based DOA estimation technique under low signal-to-interferenceplus-noise ratio (SINR) and small number of snapshots in comparison with the other comparator techniques has been validated with ULA simulated and real data. The performance of the Riemannian space-based DOA estimation for the non-ULA configuration suffered only a slight degradation for high SINR and still maintained similar performance like the ULA configuration operating in similar conditions.

The Riemannian space-based interference-plus-noise covariance (RS-INC) matrix estimation in the Riemannian space utilizes the covariance matrix for every snapshot to improve the INC estimation. The RS-INC is formulated as a linear combination of all the Hermitian positive definite matrices residing on the Riemannian manifold. The RS-INC matrix estimation technique eliminates the presence of a target in the INC covariance matrix and provides better minimum variance distortionless response (MVDR) beamforming performance in comparison with the other comparator techniques presented in this dissertation. Simulation results and results obtained with real data demonstrated the effectiveness of the Riemannian geometry-based technique under small number of snapshots and low signal-to-noise ratio scenarios.

\subsection{Limitations}

In this section, we discuss the limitations that may affect the performance of the Riemannian geometry based techniques presented in this dissertation. These limitations are : covariance matrix structure, antenna array configuration and minimum number of received range bins.

\subsubsection{Covariance Matrix Structure}

The regularized Burg algorithm discussed in this dissertation converts every range bin into Toeplitz Hermitian positive definite matrix. Any other matrix structure may affect the performance of the presented techniques. Also, other applications may have non-Toeplitz and non-Hermitian covariance matrices which make the proposed techniques not applicable. This limitation limits the Riemannian geometry based techniques to specific applications. 


\subsubsection{Antenna Array Configuration}

The Riemannian geometry based signal processing techniques presented in this dissertation are based on uniform linear antenna array. Some analysis were conducted on non-uniform linear antenna array, which resulted in 1-2 $d B$ loss in performance. Other antenna configurations like circular or planar arrays may affect the performance of the presented techniques and we may require an alternative covariance matrix estimation technique.

\subsubsection{Minimum Number of Range Bins}

The minimum number of range bins required for the Riemannian geometry based techniques to have an acceptable performance should be equal to the number of received antennas. This limitation allows the presented techniques to have sufficient sample data to estimate the covariance matrices and compute the clutter-plus-noise power. The performance of the discussed techniques may degrade if the number of received range bins is smaller than the number of received antennas.

\subsection{Future Work}

This dissertation focused on how to improve the detection and estimation in Riemannian space. The extensions of the research presented in this dissertation may focus on exploring other antenna configurations, different geometric measures, estimating the Cramér-Rao lower bound on the Riemannian manifold, identifying the micro-Doppler signature of a target in the Riemannian space and detecting and classifying targets using the Riemannian manifold learning.

\subsubsection{Antenna Configurations}

Other antenna configurations like circular, planar or 3D arrays may be explored in order to enhance the performance of the presented techniques. We may require an alternative covariance matrix estimation algorithm to convert range bins into covariance matrices and exploit other manifold structures. In addition, we may explore the variation of the phase center in randomly spaced array configurations and its effect on the direction of arrival estimation. 


\subsubsection{Geometric Means and Medians}

Other geometric measures can be used besides the Riemannian mean, median and distance. The target detection and parameter estimation in the Riemannian space could exploit different geometric measures, such as Log-Euclidean [129], Bhattacharyya [130] and Hellinger [131] distances. These geometric measures can be compared to the Riemannian measures in terms of detection and estimation performance for ULA, perturbed ULA and the non ULA configurations.

\subsubsection{Cramér-Rao Lower Bound}

The Cramér-Rao lower bound provides a fundamental limit on the accuracy of unbiased estimators. Cramér-Rao lower bound in the Riemannian space depends on the sectional curvatures of the Riemannian manifold, tensors and traces and coordinatefree extension of the Fisher information matrix [132]. It also involves theoretical aspects of the Riemannian-geometric framework. Future work could focus on deriving the Cramér-Rao lower bound on the Riemannian manifold.

\subsubsection{Micro-Doppler Signature in Riemannian Space}

Micro-Doppler analysis is often employed in identifying and recognizing drones. Micro-Doppler signatures can be studied in the Riemannian space by considering geodesic paths of the Hermitian positive definite covariance matrices on the Riemannian manifold. Fréchet's distance between geodesic paths on the Riemannian manifold could be used to estimate the micro-Doppler signature [133].

\subsubsection{Target Detection and Classification with Manifold Learning}

This dissertation considered target detection from a Riemannian space perspective and specifically, the work was focused on exploiting the Riemannian metrics to improve the probability of target detection. The Riemannian space-based target detection can be improved by using deep manifold learning which transforms the detection problem into a binary classification problem on a Riemannian manifold. Also, deep manifold learning takes advantage of the neural networks, Riemannian manifold mapping and the log-Euclidean metric. In addition, target classification can be enhanced 
by using manifold learning which exploits the statistical representations of Hermitian positive definite matrices that belong to the curved Riemannian manifold [134]. 


\section{References}

[1] D. Sathyamoorthy, "A review of security threats of unmanned aerial vehicles and mitigation steps," Journal of Defence and Security, vol. 6, no. 1, pp. 81-97, 2015 .

[2] A. H. Michel and D. Gettinger, The Drone Revolution Revisited: An Assessment of Military Unmanned Systems in 2016. Center for the Study of the Drone at Bard College, 2016.

[3] J. Mezei and A. Molnár, "Drone sound detection by correlation," in 2016 IEEE 11th International Symposium on Applied Computational Intelligence and Informatics (SACI). IEEE, May 2016, pp. 509-518.

[4] A. Mahmoud, D. Xu, and L. Xu, "Optical design of high resolution and shared aperture electro-optical/infrared sensor for UAV remote sensing applications," in 2016 IEEE International Geoscience and Remote Sensing Symposium (IGARSS), Dec. 2016, pp. 2921-2924.

[5] S. Basak and B. Scheers, "Passive radio system for real-time drone detection and DOA estimation," in 2018 International Conference on Military Communications and Information Systems (ICMCIS), Jun. 2018, pp. 1-6.

[6] T. Humphreys, "Statement on the security threat posed by unmanned aerial systems and possible countermeasures," Oversight and Management Efficiency Subcommittee, Homeland Security Committee, Washington, DC, US House, 2015.

[7] B. Nuss, L. Sit, M. Fennel, J. Mayer, T. Mahler, and T. Zwick, "MIMO OFDM radar system for drone detection," in 2017 18th International Radar Symposium (IRS), Jun. 2017, pp. 1-9.

[8] P. Wellig, P. Speirs, C. Schuepbach, R. Oechslin, M. Renker, U. Boeniger, and H. Pratisto, "Radar systems and challenges for C-UAV," in 2018 19th International Radar Symposium (IRS), Jun. 2018, pp. 1-8.

[9] R. Harmanny, J. De Wit, and G. P. Cabic, "Radar micro-Doppler feature extraction using the spectrogram and the cepstrogram," in 2014 11th European Radar Conference. IEEE, May 2014, pp. 165-168.

[10] R. Bhatia and J. Holbrook, "Riemannian geometry and matrix geometric means," Linear algebra and its applications, vol. 413, no. 2-3, pp. 594-618, 2006. 
[11] C. Li and Y. Li, "New regions including eigenvalues of Toeplitz matrices," Linear and Multilinear Algebra, vol. 62, no. 2, pp. 229-241, Feb. 2014.

[12] P. Poitevin, M. Pelletier, and P. Lamontagne, "Challenges in detecting UAS with radar," in 2017 International Carnahan Conference on Security Technology (ICCST), 2017, pp. 1-6.

[13] A. Hassanien and E. Aboutanios, "Single-snapshot beamforming using fast iterative adaptive techniques," in 2020 IEEE 11th Sensor Array and Multichannel Signal Processing Workshop (SAM), Jun. 2020, pp. 1-4.

[14] F. Barbaresco, "Information geometry manifold of Toeplitz Hermitian positive definite covariance matrices:Mostow/Berger fibration and Berezin quantization of Cartan-Siegel domains," Int. J. Emerging Trends in Signal Process. (IJETSP), vol. 1, no. 3, Mar. 2013.

[15] Y. Cheng, X. Hua, H. Wang, Y. Qin, and X. Li, "The geometry of signal detection with applications to radar signal processing," Entropy, vol. 18, no. 11, p. 381, Oct. 2016.

[16] B. Harvey and S. O'Young, "Acoustic detection of a fixed-wing UAV," Drones, vol. 2, no. 1, p. 4, 2018.

[17] L. Wang and A. Cavallaro, "Acoustic sensing from a multi-rotor drone," IEEE Sensors Journal, vol. 18, no. 11, pp. 4570-4582, 2018.

[18] L. Hauzenberger and E. Holmberg Ohlsson, "Drone detection using audio analysis," Master's thesis, Department of Electrical and Information Technology, Lund University, June 2015.

[19] S. Mandal, L. Chen, V. Alaparthy, and M. L. Cummings, "Acoustic detection of drones through real-time audio attribute prediction," in AIAA Scitech 2020 Forum, Jan 2020, pp. 1-13.

[20] A. Jara-Olmedo, W. Medina-Pazmiño, R. Mesías, B. Araujo-Villaroel, W. G. Aguilar, and J. A. Pardo, "Interface of optimal electro-optical/infrared for unmanned aerial vehicles," in International Conference of Research Applied to Defense and Security. Springer, 2018, pp. 372-380.

[21] D. Cardone and A. Meria, "New frontiers for applications of thermal infrared imaging devices: Computational psychopshysiology in the neurosciences," Sensors, vol. 17, no. 5, p. 1042, May 2017. [Online]. Available: http://dx.doi.org/10.3390/s17051042

[22] M. M. Azari, H. Sallouha, A. Chiumento, S. Rajendran, E. Vinogradov, and S. Pollin, "Key technologies and system trade-offs for detection and localization of amateur drones," IEEE Communications Magazine, vol. 56, no. 1, pp. 51-57, 2018.

[23] H. Shin, K. Choi, Y. Park, J. Choi, and Y. Kim, "Security analysis of FHSS-type drone controller," in International Workshop on Information Security Applications. Springer, 2015, pp. 240-253. 
[24] S. Alhaji Musa, R. Abdullah, R. S. Azmir, A. Sali, A. Ismail, and N. E. Abdul Rashid, "Low-slow-small (lss) target detection based on micro-Doppler analysis in forward scattering radar geometry," Sensors, vol. 19, no. 15, p. 3332, 2019 .

[25] V. Demirev, "Drone detection in urban environment the new challenge for the radar systems designers," Security \& Future, vol. 1, no. 3, pp. 114-116, 2017.

[26] M. I. Skolnik, Radar handbook. McGraw-Hill Professional, 1990.

[27] M. A. Richards, Fundamentals of radar signal processing. Tata McGraw-Hill Education, 2005.

[28] R. J. Burkholder, L. J. Gupta, and J. T. Johnson, "Comparison of monostatic and bistatic radar images," IEEE Antennas and Propagation Magazine, vol. 45, no. 3, pp. 41-50, 2003.

[29] B. R. Mahafza, Radar signal analysis and processing using MATLAB. CRC Press, 2016.

[30] V. C. Chen, The micro-Doppler effect in radar. Artech House, 2019.

[31] Richards, M.A, Fundamentals of Radar Signal Processing, 2nd ed. McGrawHill, 2014.

[32] E. Brookner, "Phased-array and radar breakthroughs," in 2007 IEEE Radar Conference, 2007, pp. 37-42.

[33] B. D. Van Veen and K. M. Buckley, "Beamforming: A versatile approach to spatial filtering," IEEE ASSP Magazine, vol. 5, no. 2, pp. 4-24, 1988.

[34] T. Jeffrey, Phased-Array Radar Design: Application of Radar Fundamentals. The Institution of Engineering and Technology, 2009.

[35] J. Li and P. Stoica, "MIMO radar with colocated antennas," IEEE Signal Processing Magazine, vol. 24, no. 5, pp. 106-114, 2007.

[36] D. Bliss and K. Forsythe, "Multiple-input multiple-output (MIMO) radar and imaging: degrees of freedom and resolution," in The Thrity-Seventh Asilomar Conference on Signals, Systems $\&$ Computers, 2003, vol. 1. IEEE, 2003, pp. $54-59$.

[37] I. Bekkerman and J. Tabrikian, "Target detection and localization using MIMO radars and sonars," IEEE Transactions on Signal Processing, vol. 54, no. 10, pp. 3873-3883, 2006.

[38] P. Sévigny, Multiple-Input Multiple Output (MIMO) Radar: Literature Survey of Papers Published between 2003 and September 2008. Defence R \& D CanadaOttawa, 2009.

[39] E. Brookner, "MIMO radars demystified - and their conventional equivalents," in 2016 IEEE International Symposium on Phased Array Systems and Technology (PAST). IEEE, 2016, pp. 1-10. 
[40] E. Conte, A. De Maio, and C. Galdi, "Signal detection in compound-Gaussian noise: Neyman-Pearson and CFAR detectors," IEEE Transactions on Signal Processing, vol. 48, no. 2, pp. 419-428, 2000.

[41] A. De Maio and S. Greco, Modern radar detection theory. The Institution of Engineering and Technology, 2016.

[42] L. E. Brennan and L. Reed, "Theory of adaptive radar," IEEE Transactions on Aerospace and Electronic Systems, no. 2, pp. 237-252, 1973.

[43] Richards, Mark A and Scheer, Jim and Holm, William A and Melvin, William L, Principles of modern radar. Citeseer, 2010.

[44] R. Austin, Unmanned aircraft systems: UAVS design, development and deployment. John Wiley \& Sons, 2011, vol. 54.

[45] J. Sharma, Applications of Small Unmanned Aircraft Systems: Best Practices and Case Studies. CRC Press, 2019.

[46] J. Burg, "Maximum entropy spectral analysis," Ph.D. dissertation, Stanford University, 1975.

[47] H. J. Landau, "Maximum entropy and maximum likelihood in spectral estimation," IEEE Transactions on Information Theory, vol. 44, no. 3, pp. 1332-1336, 1998.

[48] M. Emresoy and A. El-Jaroudi, "Evolutionary Burg spectral estimation," IEEE Signal Processing Letters, vol. 4, no. 6, pp. 173-175, 1997.

[49] F. Barbaresco, "Super-resolution spectrum analysis regularization: Burg, Capon amp; ago-antagonistic algorithms," in 1996 8th European Signal Processing Conference (EUSIPCO 1996), Sep. 1996, pp. 1-4.

[50] B. Balaji, F. Barbaresco, and A. Decurninge, "Information geometry and estimation of Toeplitz covariance matrices," in 2014 International Radar Conference, Oct. 2014, pp. 1-4.

[51] M. Arnaudon, F. Barbaresco and L. Yang, "Riemannian medians and means with applications to radar signal processing," IEEE Journal of Selected Topics in Signal Processing, vol. 7, no. 4, pp. 595-604, Aug. 2013.

[52] R. A. Horn and C. R. Johnson, Matrix analysis. Cambridge university press, 2012.

[53] F. Zhang, Matrix theory: basic results and techniques. Springer Science \& Business Media, 2011.

[54] H. Zhou, "On some trace inequalities for positive definite Hermitian matrices," Journal of Inequalities and Applications, vol. 2014, no. 1, p. 64, 2014.

[55] W. Kühnel, Differential geometry. American Mathematical Soc., 2015. 
[56] S. E. Kozlov, "Geometry of the real Grassmannian manifolds. parts I, II," $Z a$ piski Nauchnykh Seminarov POMI, vol. 246, pp. 84-107, 1997.

[57] B.-y. Chen, "Differential geometry of real submanifolds in a Kähler manifold," Monatshefte für Mathematik, vol. 91, no. 4, pp. 257-274, 1981.

[58] W. M. Boothby, An Introduction to Differentiable Manifolds and Reimannian Geometry. Academic Press, 2002.

[59] P. Gilkey, J. Park, and R. Vazquez-Lorenzo, Aspects of Differential Geometry I. Morgan \& Claypool, 2015.

[60] A. Barachant, S. Bonnet, M. Congedo, and C. Jutten, "Multiclass brain-computer interface classification by Riemannian geometry," IEEE Transactions on Biomedical Engineering, vol. 59, no. 4, pp. 920-928, Oct. 2012.

[61] M. Moakher, "A differential geometric approach to the geometric mean of symmetric positive-definite matrices," SIAM Journal on Matrix Analysis and Applications, vol. 26, no. 3, pp. 735-747, Jan. 2005.

[62] J. M. Lee, Introduction to Riemannian manifolds. Springer, 2018.

[63] M. Tang, Y. Rong, and J. Zhou, "An information geometric viewpoint on the detection of range distributed targets," Mathematical Problems in Engineering, vol. 2015, pp. 1-9, 2015.

[64] M. Oudin, J.-P. Delmas, F. Barbaresco, and L. Lupinski, "Siegel distance-based covariance matrix selection for space-time adaptive," in RADAR 2009: International Radar Conference" Surveillance for a safer world", 2009.

[65] X. Pennec, S. Sommer, and T. Fletcher, Riemannian geometric statistics in medical image analysis. Academic Press, 2019.

[66] P. T. Fletcher, S. Venkatasubramanian, and S. Joshi, "Robust statistics on Riemannian manifolds via the geometric median," in 2008 IEEE Conference on Computer Vision and Pattern Recognition, Jun. 2008, pp. 1-8.

[67] M.-T. Chien, S.-T. Fang, and Y.-X. Su, "A generalization of Gershgorin circles," Applied and Computational Mathematics, vol. 15, no. 1, pp. 101-111, Jan. 2016.

[68] W. Zhang and C. Li, "An eigenvalue inclusion set for matrices with a constant main diagonal entry," Symmetry, vol. 10, no. 12, p. 745, Dec. 2018.

[69] A. Melman, "Modified Gershgorin disks for companion matrices," SIAM Journal on Matrix Analysis and Applications, vol. 54, pp. 355-373, Mar. 2012.

[70] S. Li, C. Li, and Y. Li, "Exclusion sets for eigenvalues of matrices," arXiv preprint arXiv:1705.01758, May 2017.

[71] N. Amrouche, A. Khenchaf, and D. Berkani, "Tracking and detecting moving weak targets," Advances in Science, Technology and Engineering Systems Journal, vol. 3, no. 1, pp. 467-471, Jan. 2018. 
[72] R. Vicen-Bueno, M. Rosa-Zurera, L. Cuadra-Rodriguez, and D. De La MataMoya, "Models of radar clutter from the Weibull distribution," in Proc. of the 5th WSEAS Int. Conf. on Signal Process., Robot. and Automat., Feb. 2006, pp. $376-380$.

[73] K. D. Ward, R. J. A. Tough, and S. Watts, "Sea clutter: Scattering, the K-distribution and radar performance," Waves in Random and Complex Media, vol. 17, no. 2, pp. 233-234, 2007. [Online]. Available: https://doi.org/10.1080/17455030601097927

[74] F. D. Almeida García, A. C. Flores Rodriguez, G. Fraidenraich, and J. C. S. Santos Filho, "CA-CFAR detection performance in homogeneous Weibull clutter," IEEE Geoscience and Remote Sensing Letters, vol. 16, no. 6, pp. 887-891, 2019 .

[75] J. Lapuyade-Lahorgue and F. Barbaresco, "Radar detection using Siegel distance between autoregressive processes, application to HF and X-band radar," in 2008 IEEE Radar Conference, May 2008, pp. 1-6.

[76] J. H. Shin and Y. Choi, "Robust control for the detection threshold of CFAR process in cluttered environments," Sensors, vol. 20, no. 14, p. 3904, 2020.

[77] X. Hua, Y. Cheng, H. Wang, Y. Qin, Y. Li, and W. Zhang, "Matrix cfar detectors based on symmetrized KullbackLeibler and total KullbackLeibler divergences," Digit. Signal Process., vol. 69, no. C, p. 106-116, Oct. 2017.

[78] X. Hua, Y. Cheng, H. Wang, Y. Qin, and Y. Li, "Geometric means and medians with applications to target detection," IET Signal Processing, vol. 11, no. 6, pp. 711-720, Mar. 2017.

[79] T. M. Cover and J. A. Thomas, Elements of information theory. John Wiley \& Sons, 2012.

[80] S.-I. Amari, "Differential geometry of curved exponential families-curvatures and information loss," The Annals of Statistics, vol. 10, pp. 357-385, 1982.

[81] M. S. Pinsker, Information and information stability of random variables and processes. Holden-Day, 1964.

[82] S. Kullback, Information theory and statistics. Courier Corporation, 1997.

[83] Z. Chebbi and M. Moakher, "Means of Hermitian positive-definite matrices based on the log-determinant $\alpha$-divergence function," Linear Algebra and its Applications, vol. 436, no. 7, pp. 1872-1889, Feb. 2012.

[84] A. Daniilidis, R. Deville, E. Durand-Cartagena, L. Rifford, "Self-contracted curves in Riemannian manifolds," Journal of Mathematical Analysis and Applications, vol. 457, pp. 1333-1352, Jan. 2018.

[85] H. Krim and M. Viberg, "Two decades of array signal processing research: the parametric approach," IEEE Signal Processing Magazine, vol. 13, no. 4, pp. 67-94, Jul. 1996. 
[86] H. L. Van Trees, Optimum array processing: Part IV of detection, estimation, and modulation theory. John Wiley \& Sons, 2004.

[87] Z. Chen, G. Gokeda, and Y. Yu, Introduction to Direction-of-arrival Estimation. Artech House, 2010.

[88] E. Fishler, M. Grosmann, and H. Messer, "Detection of signals by information theoretic criteria: General asymptotic performance analysis," IEEE Transactions on Signal Processing, vol. 50, no. 5, pp. 1027-1036, Aug. 2002.

[89] S. Chandran, Advances in direction-of-arrival estimation. Artech House Boston, 2006.

[90] J. Capon, "High-resolution frequency-wavenumber spectrum analysis," Proceedings of the IEEE, vol. 57, no. 8, pp. 1408-1418, Aug. 1969.

[91] F. Akbari, S. S. Moghaddam, and V. T. Vakili, "MUSIC and MVDR DOA estimation algorithms with higher resolution and accuracy," in 2010 5th International Symposium on Telecommunications. IEEE, Dec. 2010, pp. 76-81.

[92] S. Shirvani-Moghaddam and S. Almasi-Monfared, "A comprehensive performance study of narrowband DOA estimation algorithms," International Journal on Communications Antenna and Propagation (IRECAP), vol. 1, pp. 396-405, Aug. 2011.

[93] S. S. Haykin, Adaptive filter theory. Pearson Education India, 2008.

[94] C. Vaidyanathan and K. Buckley, "Performance analysis of the MVDR spatial spectrum estimator," IEEE Transactions on Signal Processing, vol. 43, no. 6, pp. 1427-1437, Jun. 1995.

[95] G. Bienvenu and L. Kopp, "Principe de la goniométrie passive adaptative," in Proc. 7th GRESIT Symposium, vol. 106, May 1979, pp. 1-7.

[96] R. Schmidt, "Multiple emitter location and signal parameter estimation," IEEE Transactions on Antennas and Propagation, vol. 34, no. 3, pp. 276-280, Mar. 1986.

[97] R. Roy and T. Kailath, "ESPRIT estimation of signal parameters via rotational invariance techniques," IEEE Transactions on Acoustics, Speech, and Signal Processing, vol. 37, no. 7, pp. 984-995, Jul. 1989.

[98] N. Waweru , D. Konditi and P. Langat, "Performance analysis of MUSIC, root-MUSIC and ESPRIT DOA estimation algorithm," International Journal of Electronics and Communication Engineering, vol. 8, no. 1, pp. 209-216, Mar. 2014.

[99] Z. Xiaofei, L. Wen, S. Ying, Z. Ruina, and X. Dazhuan, "A novel DOA estimation algorithm based on eigen-space," in 2007 International Symposium on Microwave, Antenna, Propagation and EMC Technologies for Wireless Communications. IEEE, Aug. 2007, pp. 551-554. 
[100] M. Rani, S. B. Dhok, and R. B. Deshmukh, "A systematic review of compressive sensing: Concepts, implementations and applications," IEEE Access, vol. 6, pp. 4875-4894, Jan. 2018.

[101] Y. Wang, G. Leus, and A. Pandharipande, "Direction estimation using compressive sampling array processing," in 2009 IEEE/SP 15th Workshop on Statistical Signal Processing. IEEE, Aug. 2009, pp. 626-629.

[102] X. Xu, X. Wei, and Z. Ye, "DOA estimation based on sparse signal recovery utilizing weighted $\ell_{1}$-norm penalty," IEEE signal processing letters, vol. 19, no. 3, pp. 155-158, Jan. 2012.

[103] C. Zheng, G. Li, H. Zhang, and X. Wang, "An approach of DOA estimation using noise subspace weighted $\ell_{1}$-minimization," in 2011 IEEE International Conference on Acoustics, Speech and Signal Processing (ICASSP). IEEE, May 2011, pp. 2856-2859.

[104] B. Liu, S.-y. Matsushita, and L. Xu, "DOA estimation with small snapshots using weighted mixed norm based on spatial filter," IEEE Transactions on Vehicular Technology, vol. 69, no. 12, pp. 16183-16187, Oct 2020.

[105] H. Chahrour, R. Dansereau, S. Rajan, and B. Balaji, "Direction of arrival estimation using Riemannian mean and distance," in 2019 IEEE Radar Conference (RadarConf), May 2019, pp. 1-5.

[106] S. Amari, "Information geometry on hierarchy of probability distributions," IEEE Transactions on Information Theory, vol. 47, no. 5, pp. 1701-1711, July 2001.

[107] S. Lang, Fundamentals of Differential Geometry. Springer, 1999.

[108] K. M. Wong, J. Zhang, J. Liang and H. Jiang, "Mean and median of PSD matrices on a Riemannian manifold: Application to detection of narrow-band sonar signals," IEEE Transactions on Signal Processing, vol. 65, no. 24, pp. 6536-6550, Dec. 2017.

[109] M. Arnaudon, F. Barbaresco and L. Yang, Medians and Means in Riemannian Geometry: Existence Uniqueness and Computation Matrix Information Geometry. USA, NY, New York: Springer, 2012.

[110] M. Coutino, R. Pribic and G. Leus, "Direction of arrival estimation based on information geometry," in 2016 IEEE International Conference on Acoustics, Speech and Signal Processing (ICASSP), March 2016, pp. 3066-3070.

[111] J. Li and P. Stoica, Robust adaptive beamforming. Wiley Online Library, 2006.

[112] L. Du, J. Li, and P. Stoica, "Fully automatic computation of diagonal loading levels for robust adaptive beamforming," IEEE Transactions on Aerospace and Electronic Systems, vol. 46, no. 1, pp. 449-458, Feb. 2010.

[113] A. Gershman, "Robust adaptive beamforming: An overview of recent trends and advances in the field," in 4th International Conference on Antenna Theory and Techniques (Cat. No. 03EX699), vol. 1. IEEE, Sep. 2003, pp. 30-35. 
[114] Y. Xiao, J. Yin, H. Qi, H. Yin, and G. Hua, "MVDR algorithm based on estimated diagonal loading for beamforming," Mathematical Problems in Engineering, vol. 2017, Jan. 2017.

[115] O. Ledoit and M. Wolf, "A well-conditioned estimator for large-dimensional covariance matrices," Journal of multivariate analysis, vol. 88, no. 2, pp. 365411, Feb. 2004.

[116] M. Rahmani and G. K. Atia, "A subspace method for array covariance matrix estimation," in 2016 IEEE Sensor Array and Multichannel Signal Processing Workshop (SAM), Jul. 2016, pp. 1-5.

[117] Y. Gu, N. A. Goodman, Y. D. Zhang, A. De Maio, Y. Eldar, and A. Haimovich, Compressed Sensing in Radar Signal Processing. Cambridge Univ. Press, 2019.

[118] H. Chahrour, R. Dansereau, S. Rajan, and B. Balaji, "Improved covariance matrix estimation using Riemannian geometry for beamforming applications," in 2020 IEEE International Radar Conference (RADAR), May 2020, pp. 693697.

[119] G. Charis and N. Showme, "Beamforming in wireless communication standards: a survey," Indian Journal of Science and Technology, vol. 10, p. 5, Feb. 2017.

[120] T. S. Kiong, S. B. Salem, J. K. S. Paw, K. P. Sankar, and S. Darzi, "Minimum variance distortionless response beamformer with enhanced nulling level control via dynamic mutated artificial immune system," The Scientific World Journal, vol. 5, pp. 1-9, Jan. 2014.

[121] B. D. Carlson, "Covariance matrix estimation errors and diagonal loading in adaptive arrays," IEEE Transactions on Aerospace and Electronic systems, vol. 24, no. 4, pp. 397-401, Jul. 1988.

[122] Z. Xiao, J. Wang, L. Geng, F. Zhang, and J. Tong, "On the robustness of covariance matrix shrinkage-based robust adaptive beamforming," in Proceedings of the 2018 International Conference on Electronics and Electrical Engineering Technology, 2018, pp. 196-201.

[123] S. S. Chen, D. L. Donoho, and M. A. Saunders, "Atomic decomposition by basis pursuit," SIAM Journal on Matrix Analysis and Applications, vol. 43, no. 1, pp. 129-159, 2001.

[124] H. Cox, R. Zeskind, and M. Owen, "Robust adaptive beamforming," IEEE Transactions on Acoustics, Speech, and Signal Processing, vol. 35, no. 10, pp. 1365-1376, Oct. 1987.

[125] L. Chang and C. Yeh, "Performance of DMI and eigenspace-based beamformers," IEEE Transactions on Antennas and Propagation, vol. 40, no. 11, pp. 1336-1347, Nov. 1992.

[126] C. Gierull, "A fast subspace estimation method for adaptive beamforming based on covariance matrix transformation." AEU International Journal of Electronics and Communications., vol. 5, pp. 196-205, 1997. 
[127] X. Ru, Z. Liu, and W. Jiang, "Normalized residual-based outlier detection," in 2014 IEEE International Conference on Signal Processing, Communications and Computing (ICSPCC). IEEE, Aug. 2014, pp. 190-193.

[128] M. Grant and S. Boyd, "Cvx: Matlab software for disciplined convex programming, version 2.1," 2014.

[129] V. Arsigny, P. Fillard, X. Pennec, and N. Ayache, "Fast and simple calculus on tensors in the log-Euclidean framework," in International Conference on Medical Image Computing and Computer-Assisted Intervention. Springer, 2005, pp. $115-122$.

[130] M. Charfi, Z. Chebbi, M. Moakher, and B. C. Vemuri, "Using the Bhattacharyya mean for the filtering and clustering of positive-definite matrices," in International Conference on Geometric Science of Information. Springer, 2013, pp. $551-558$.

[131] H. M. Anver, Mean Hellinger distance as an error criterion in univariate and multivariate kernel density estimation. Southern Illinois University at Carbondale, 2010.

[132] J. Xavier and V. Barroso, "Intrinsic variance lower bound (IVLB): an extension of the Cramér-Rao bound to Riemannian manifolds," in Proceedings.(ICASSP'05). IEEE International Conference on Acoustics, Speech, and Signal Processing, 2005., vol. 5. IEEE, 2005, pp. v-1033.

[133] F. Barbaresco, "Radar detection for non-stationary time-Doppler signal based on Fréchet distance of geodesic curves on covariance matrix information geometry manifold," in 2013 14th International Radar Symposium (IRS), vol. 1. IEEE, 2013, pp. 307-312.

[134] D. Brooks, O. Schwander, F. Barbaresco, J.-Y. Schneider, and M. Cord, "Deep learning and information geometry for drone micro-Doppler radar classification," in 2020 IEEE Radar Conference (RadarConf20). IEEE, 2020, pp. 1-6.

[135] F. M. Han and X. D. Zhang, "An ESPRIT like algorithm for coherent DOA estimation," IEEE Antennas and Wireless Propagation Letters, vol. 4, no. 5, pp. 443-446, Dec. 2005.

[136] O. A. Oumar, M. F. Siyau, and T. P. Sattar, "Comparison between MUSIC and ESPRIT direction of arrival estimation algorithms for wireless communication systems," in The First International Conference on Future Generation Communication Technologies. IEEE, May 2012, pp. 99-103.

[137] L. Liu, X. Zhang, and P. Chen, "Compressed sensing-based DOA estimation with antenna phase errors," Electronics, vol. 8, no. 3, p. 294, Mar. 2019.

[138] D. J. Tylavsky and G. R. Sohie, "Generalization of the matrix inversion lemma," Proceedings of the IEEE, vol. 74, no. 7, pp. 1050-1052, Jul. 1986.

[139] C. Stein, "Estimation of a covariance matrix," in 39th Annual Meeting IMS, Atlanta, GA, 1975. 
[140] W. James and C. Stein, "Estimation with quadratic loss," in Breakthroughs in statistics. Springer, 1992, vol. 1, pp. 443-460.

[141] Y. Chen, A. Wiesel, Y. C. Eldar, and A. O. Hero, "Shrinkage algorithms for MMSE covariance estimation," IEEE Transactions on Signal Processing, vol. 58, no. 10, pp. 5016-5029, Jun. 2010.

[142] Y. Gu and Y. D. Zhang, "Adaptive beamforming based on interference covariance matrix estimation," in 53rd Asilomar Conference on Signals, Systems, and Computers. IEEE, Nov. 2019, pp. 619-623.

[143] D. Malioutov, M. Cetin, and A. S. Willsky, "A sparse signal reconstruction perspective for source localization with sensor arrays," IEEE Transactions on Signal Processing, vol. 53, no. 8, pp. 3010-3022, Jul. 2005. 


\section{Appendix A}

\section{DOA Estimation Techniques}

This Appendix provides a brief description of the DOA estimation techniques discussed in this thesis.

\section{A.1 Conventional DOA Estimation}

Beamforming and null steering are two conventional methods used for DOA estimation, which steer the beams electronically in all possible directions looking for peaks in the output power [89]. The conventional methods discussed here are the delayand-sum method (classical beamformer) and the Capon's minimum variance method.

\section{A.1.1 Delay and Sum Technique}

The delay and sum (DAS) method, also referred to as the classical beamformer method or Fourier method, is one of the simplest methods for DOA estimation [111]. The delay and sum technique is based on combining the received signals linearly using complex weights as shown in Fig. A.1. The output signal $\mathbf{y}(k)$ can be written as

$$
\mathbf{y}(k)=\mathbf{w}^{H} \mathbf{z}(k)
$$

The total output power $P_{b}$ of the DAS method can be expressed as

$$
\begin{aligned}
P_{b} & =E\left[|\mathbf{y}(k)|^{2}\right] \\
& =E\left[\left|\mathbf{w}^{H} \mathbf{z}(k)\right|^{2}\right]=\mathbf{w}^{H} E\left[\mathbf{z}(k) \mathbf{z}(k)^{H}\right] \mathbf{w} \\
& =\mathbf{w}^{H} \mathbf{R}_{z z} \mathbf{w} .
\end{aligned}
$$


Consider a signal $\mathbf{z}(k)$ impinging on the antenna array at an angle $\theta_{0}$. Using the system model (4.1) discussed in Sec. 4.1, the power of the beamformer output can expressed as

$$
\begin{aligned}
P_{b} & =E\left[\left|\mathbf{w}^{H}\left(\mathbf{a}\left(\theta_{0}\right) \mathbf{s}(k)+\mathbf{n}(k)\right)\right|^{2}\right] \\
& =\left(\left|\mathbf{w}^{H} \mathbf{a}\left(\theta_{0}\right)\right|\right)\left(\sigma_{s}^{2}+\sigma_{n}^{2}\right)
\end{aligned}
$$

where $\mathbf{a}\left(\theta_{0}\right)$ is the steering vector associated with the DOA angle $\theta_{0}, \mathbf{n}(k)$ is the noise vector at the array input, and $\sigma_{s}^{2}$ and $\sigma_{n}^{2}$ are the signal power and noise power respectively. It can be seen from (A.3) that the output power is maximized when $\mathbf{w}=\mathbf{a}\left(\theta_{0}\right)$. The receiver antenna has the highest gain in the direction of $\theta_{0}$ when $\mathbf{w}=\mathbf{a}\left(\theta_{0}\right)$ due to the fact that the signal phases are aligned which allow them to be added constructively.

The output power of the DAS method can be written in terms of the angle of arrival as

$$
P_{b}=\mathbf{w}^{H} \mathbf{R}_{z z} \mathbf{w}=\mathbf{a}^{H}(\theta) \mathbf{R}_{z z} \mathbf{a}(\theta)
$$

. The output power as a function of the angle of arrival $\theta$ is called the spatial spectrum and the DOA can be estimated by locating the peaks in the spatial spectrum in (A.4).

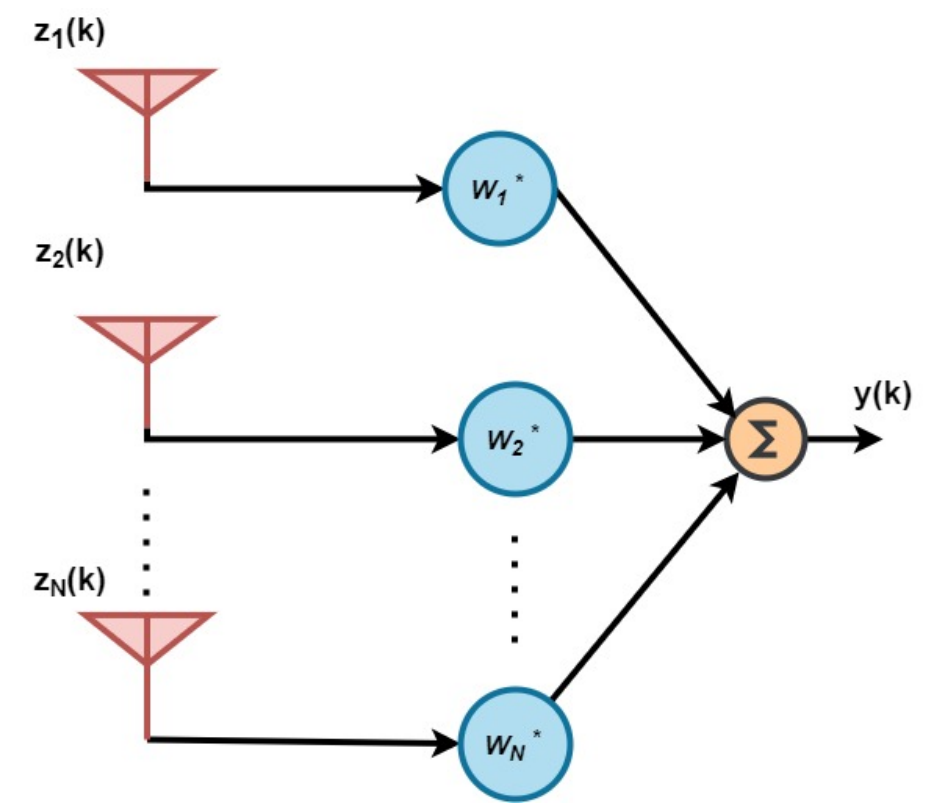

Figure A.1: Illustration of the classical beamforming structure. 
The delay and sum method is easy to implement and does not require a lot of computations. On the other hand, the width of the beam and the height of the sidelobes from multiple directions limits its performance and reduces the resolution. In addition, the DAS technique requires a large number of antenna elements to achieve high resolution.

\section{A.1.2 Minimum Variance Distortionless Response}

The MVDR technique can be expressed as the optimization problem [92]

$$
\begin{array}{lr}
\min _{\mathbf{w}} & \mathbf{w}^{H} \mathbf{R}_{z z} \mathbf{w} \\
\text { s.t. } & \mathbf{w}^{H} \mathbf{a}\left(\theta_{0}\right)=1 .
\end{array}
$$

The solution for this optimization problem can be obtained by minimizing the variance (average power) of the output signal while passing the signal arriving in the look direction without distortion with unity gain and zero phase shift. (A.5) represents a constraint optimization problem which can be solved using the method of Lagrange multipliers. This approach exploits least squares techniques to determine the solution. Using a Lagrange multiplier, the weight vector $w$ that can be shown to be [93]

$$
\mathbf{w}=\frac{\mathbf{R}_{z z}^{-1} \mathbf{a}(\theta)}{\mathbf{a}^{H}(\theta) \mathbf{R}_{z z}^{-1} \mathbf{a}(\theta)}
$$

The output power of the array as a function of the angle of arrival using the MVDR technique is given by the Capon's spatial spectrum [90]

$$
P_{m v d r}(\theta)=\frac{1}{\mathbf{a}^{H}(\theta) \mathbf{R}_{z z}^{-1} \mathbf{a}(\theta)}
$$

The DOAs are estimated by locating the peaks in Capon's spatial spectrum over the whole range of $\theta$. MVDR provides a better resolution when compared to the DAS method. However, the performance of MVDR degrades if other received signals are correlated with the signal of interest. In addition, MVDR suffers from self nulling in the case of DOA mismatch [94]. 


\section{A.2 Subspace Based DOA Estimation}

The geometric concepts upon which MUSIC is founded form the basis for a much broader class of subspace-based algorithms [96]. In addition to the MUSIC algorithm, another subspace-based algorithm called estimation of signal parameters via rotational invariance technique (ESPRIT) was proposed by Roy [97].

\section{A.2.1 MUSIC Algorithm}

The MUSIC algorithm is based on exploiting the eigen-structure of the input covariance matrix. Using the narrowband signal model in Section 4.1 and based on $L$ signals incident on the array, the received input data vector can be expressed as a linear combination of the $L$ incident waveforms and noise. That is [89],

$$
\begin{gathered}
{\left[\begin{array}{c}
z_{1} \\
\vdots \\
z_{N}
\end{array}\right]=\left[\mathbf{a}\left(\theta_{1}\right) \cdots \mathbf{a}\left(\theta_{L}\right)\right]\left[\begin{array}{c}
s_{1} \\
\vdots \\
s_{L}
\end{array}\right]+\left[\begin{array}{c}
n_{1} \\
\vdots \\
n_{N}
\end{array}\right]} \\
\mathbf{z}=\mathbf{A} \mathbf{s}+\mathbf{n}
\end{gathered}
$$

where $\mathbf{s}=\left[s_{1} \ldots s_{L}\right]$ is the vector of incident signals, $\mathbf{n}=\left[n_{1} \ldots n_{L}\right]$ is the noise vector, $\mathbf{a}\left(\theta_{l}\right)$ is the array steering vector corresponding to the direction of arrival of the $l^{\text {th }}$ signal and $\mathbf{A}$ is the array manifold matrix. In geometric terms, the received vector $\mathbf{z}$ and the steering vectors $\mathbf{a}\left(\theta_{l}\right)$ can be visualized as vectors in $N$ dimensional space. From (A.8), it can be seen that the received vector $\mathbf{z}$ is a linear combination of the array steering vectors, with $s_{1}, s_{2} \ldots, s_{L}$ being the weights of the linear combination operator. The input covariance matrix of the received data signal $\mathbf{R}_{z z}$ can be expressed as

$$
\begin{aligned}
\mathbf{R}_{z z} & =E\left[\mathbf{z z}^{H}\right]=\mathbf{A} E\left[\mathbf{s s}^{H}\right] \mathbf{A}^{H}+E\left[\mathbf{n n}^{H}\right] \\
& =\mathbf{A} \mathbf{R}_{s s} \mathbf{A}^{H}+\sigma_{n}^{2} \mathbf{I}
\end{aligned}
$$


where $\mathbf{R}_{s s}$ is the incident signal correlation matrix, $\sigma_{n}^{2}$ is the noise power and $\mathbf{I}$ is the identity matrix of size $N \times N$. The vector columns of $\mathbf{A}$ are linearly independent and $\mathbf{R}_{s s}$ is non singular since incident signals are uncorrelated or partially correlated.

The non-singularity of $\mathbf{R}_{s s}$ and the full rank of $\mathbf{A}$ guarantees that $\mathbf{A} \mathbf{R}_{s s} \mathbf{A}^{H}$ is positive semi-definite with rank $L$. The implies that $N-L$ of its eigenvalues are zeros. $\lambda_{1}, \lambda_{2} \ldots, \lambda_{N}$ and $\mathbf{v}_{1}, \mathbf{v}_{2}, \ldots, \mathbf{v}_{N}$ correspond to the eigenvalues and eigenvectors of $\mathbf{R}_{z z}$. It can be seen from (A.11) that the $N-L$ smallest eigenvalues of $\mathbf{R}_{z z}$ are all equal to $\sigma_{n}^{2}$, that is

$$
\lambda_{L+1}=\lambda_{L+2}=\cdots=\lambda_{M}=\lambda_{\min }=\sigma_{n}^{2} .
$$

All the eigenvalues corresponding to the noise power will not be identical since the autocorrelation $\mathbf{R}_{z z}$ is estimated from a finite data sample size. Once the multiplicity $K$ of the smallest eigenvalue is determined, an estimate of the number of signals, $\hat{L}$, can be obtained from the relation $N=L+K$. Therefore, the estimated number of signals is given by

$$
\hat{L}=N-K \text {. }
$$

From the definition of eigenvalues and eigenvectors, the eigenvector corresponding to the smallest eigenvalues (non principle eigenvalues) must satisfy

$$
\mathbf{R}_{z z} \mathbf{v}_{i}=\sigma_{n}^{2} \mathbf{v}_{i}, \quad i=L+1, \ldots, M .
$$

Substituting (A.14) in (A.11) gives

$$
\mathbf{A R}_{s s} \mathbf{A}^{H} \mathbf{v}_{i}=0, \quad i=L+1, \ldots, M .
$$

Since $\mathbf{A}$ is full rank and $\mathbf{R}_{s s}$ is nonsingular, it follows that

$$
\mathbf{A}^{H} \mathbf{v}_{i}=0, \quad i=L+1, \ldots, M
$$

Since the dot product of $\mathbf{A}^{H}$ and the eigenvectors $\mathbf{v}_{L+1}, \ldots, \mathbf{v}_{M}$ are zero, it implies that the column vectors of $\mathbf{A}^{H}$ are perpendicular to those eigenvectors such that

$$
\left\{\mathbf{a}\left(\theta_{1}\right), \ldots, \mathbf{a}\left(\theta_{L}\right)\right\} \perp\left\{\mathbf{v}_{L+1}, \ldots, \mathbf{v}_{M}\right\}
$$


This proves that the eigenvectors of the covariance matrix $\mathbf{R}_{z z}$ contains two orthogonal subspaces called the principal eigen-subspace (signal subspace) and the non-principal eigen-subspace (noise subspace). The steering vectors corresponding to the direction of arrivals of signals lie in the signal subspace and are orthogonal to the noise subspace. By searching through all possible array steering vectors to find those which are perpendicular to the space spanned by the non-principal eigenvectors, the direction of arrivals $\theta_{l}$ can be determined.

The direction of arrival of the multiple incident signals can be estimated by locating the peaks of a MUSIC spatial spectrum given by [98]

$$
P_{M U S I C}(\theta)=\frac{1}{\mathbf{a}^{H}(\theta) \mathbf{V}_{n} \mathbf{V}_{n}^{H} \mathbf{a}(\theta)}
$$

or

$$
P_{M U S I C}(\theta)=\frac{\mathbf{a}^{H}(\theta) \mathbf{a}(\theta)}{\mathbf{a}^{H}(\theta) \mathbf{V}_{n} \mathbf{V}_{n}^{H} \mathbf{a}(\theta)}
$$

where $\mathbf{V}_{n}=\mathbf{v}_{L+1}, \ldots, \mathbf{v}_{M}$. The denominator of (A.18) and (A.19) is essentially a scalar measure of the distance between the steering vectors $\mathbf{a}(\theta)$ in the array manifold and the estimated noise subspace spanned by the eigenvectors $\mathbf{V}_{n}$. The product $\mathbf{V}_{n} \mathbf{V}_{n}^{H}$ represents the projection matrix on the noise subspace. The orthogonality between $\mathbf{a}(\theta)$ and $\mathbf{V}_{n}$ will minimize the denominator and hence peaks will appear in the MUSIC spectrum defined in (A.18) and (A.19). The $\hat{L}$ largest peaks in the MUSIC spectrum correspond to the DOA of the signals impinging on the array.

Figure A.2 shows a comparison between the resolution performance of MUSIC and the MVDR method. As seen clearly from the plot, MUSIC can resolve closely spaced signals which cannot be detected by the MVDR method

It should be noted that, unlike the conventional methods, the MUSIC spatial spectrum does not estimate the signal power associated with each arrival angle. The peaks of $P_{M U S I C}(\theta)$ are guaranteed to correspond to the true angles of arrival.

\section{A.2.2 ESPRIT Algorithm}

The goal of the ESPRIT technique is to exploit the rotational invariance in the signal subspace which is created by two equal size identical arrays with a translational 


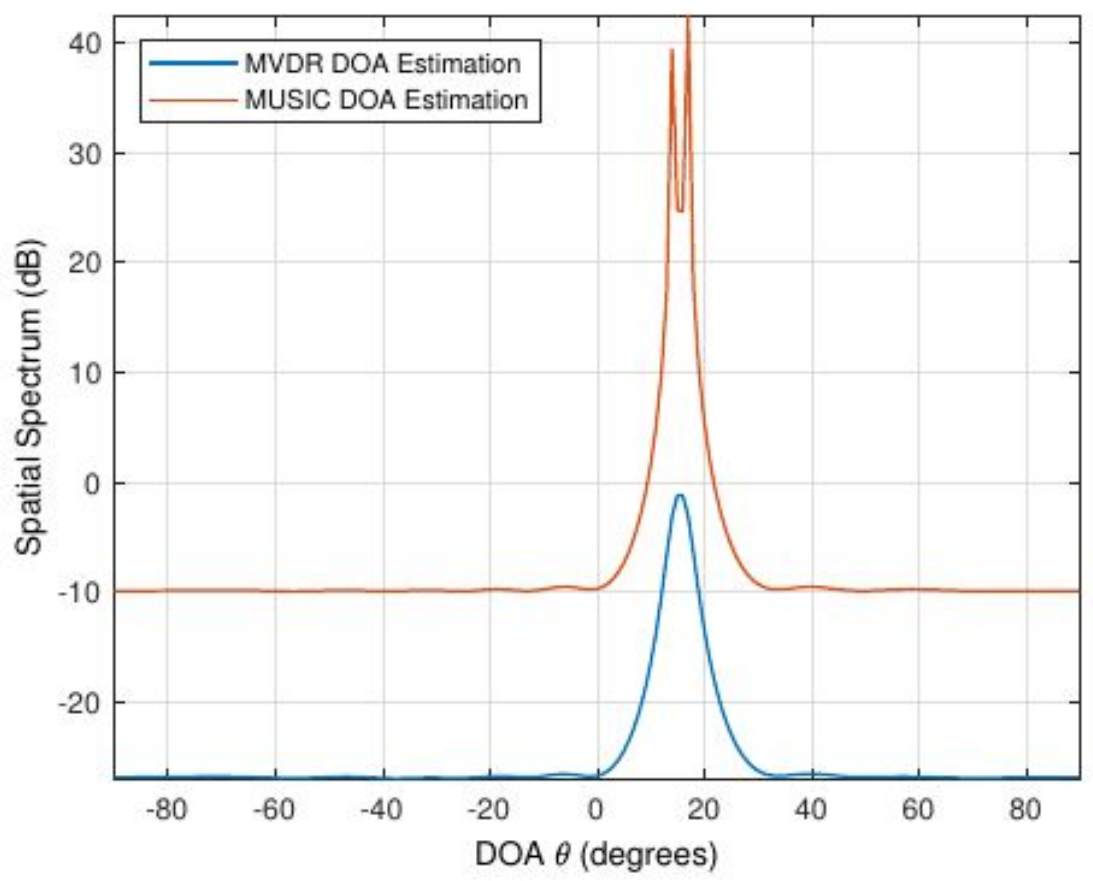

Figure A.2: Comparison of MVDR and MUSIC estimation methods. Two signals of equal power at an SNR of $20 \mathrm{~dB}$ arrive at an 8-element uniformly spaced array with an inter-element spacing equal to half a wavelength at angles 15 and 17 degrees respectively.

invariance structure. The array should possess a displacement (translational) invariance, and the sensors should occur in matched pairs with identical displacement [97]. There are many practical applications where these conditions are satisfied, such as in the case of a uniform linear array.

To describe mathematically the effect of the translational invariance of the sensor array, it is convenient to describe the array as being composed of two identical subarrays $\mathbf{Z}_{0}$ and $\mathbf{Z}_{1}$ physically displaced (not rotated) from each other by a known displacement (translational) vector $\delta$. Consider a ULA array consists of $N$ elements and can be composed of $n=N / 2$ sensor pairs or doublets. The signals received at the $i^{\text {th }}$ doublet can then be expressed as $[89,135]$

$$
z_{0 i}=\sum_{k=1}^{D} \mathbf{s}_{k}(t) \mathbf{a}_{i}\left(\theta_{k}\right)+\mathbf{n}_{0 i}(t)
$$




$$
z_{1 i}=\sum_{k=1}^{D} \mathbf{s}_{k}(t) \exp \left[j \omega_{0} \Delta \cos \left(\frac{\theta_{k}}{c}\right)\right] \mathbf{a}_{i}\left(\theta_{k}\right)+\mathbf{n}_{1 i}(t)
$$

where $\theta_{k}$ is the direction of arrival of the $k^{t h}$ source relative to the direction of the translational vector $\Delta$ and $D$ is the number of signals incident on the array. The received signal vector at the two subarrays can be written in matrix notation

$$
\begin{gathered}
\mathbf{z}_{0}=\mathbf{A} \mathbf{s}(t)+\mathbf{n}_{0}(t) \\
\mathbf{z}_{1}=\mathbf{A} \Phi \mathbf{s}(t)+\mathbf{n}_{1}(t)
\end{gathered}
$$

where $\boldsymbol{\Phi}$ is a $D \times D$ diagonal unitary matrix whose diagonal elements represent the phase delays between the doublet sensors for the $D$ signals. The matrix $\boldsymbol{\Phi}$ relates the measurements from subarray $\mathbf{Z}_{0}$ to those from subarray $\mathbf{Z}_{1}$ which can written as

$$
\Phi=\operatorname{diag}\left[\exp \left(j \gamma_{1}\right), \exp \left(j \gamma_{2}\right), \ldots, \exp \left(j \gamma_{D}\right)\right]
$$

where

$$
\gamma_{k}=\frac{\omega_{0} \Delta}{c} \cos \left(\theta_{k}\right)
$$

The total array vector $\mathbf{z}(t)$ can be written as

$$
\mathbf{z}(t)=\left[\begin{array}{l}
\mathbf{z}_{0}(t) \\
\mathbf{z}_{1}(t)
\end{array}\right]=\overline{\mathbf{A}} \mathbf{s}(t)+\mathbf{n}(t)
$$

where

$$
\overline{\mathbf{A}}=\left[\begin{array}{c}
\mathbf{A} \\
\mathbf{A} \boldsymbol{\Phi}
\end{array}\right] \text { and } \mathbf{n}(t)=\left[\begin{array}{l}
\mathbf{n}_{0}(t) \\
\mathbf{n}_{1}(t)
\end{array}\right] .
$$

ESPRIT exploits the rotational invariance of the underlying signal subspace induced by the translational invariance of the sensor array [97]. The signal subspace is the one that contains the outputs from the two subarrays $\mathbf{Z}_{0}$ and $\mathbf{Z}_{1}$. Sampling the output of the arrays simultaneously leads to two sets of vectors $\mathbf{V}_{0}$ and $\mathbf{V}_{1}$, that span the same signal subspace. 
The signal subspace can be obtained from the knowledge of the input covariance matrix $\mathbf{R}_{z z}=\mathbf{A} \mathbf{R}_{s s} \mathbf{A}^{H}+\sigma_{n}^{2} \mathbf{I}$. If $D \leqslant n$, the $M-D$ smallest eigenvalues of $\mathbf{R}_{z z}$ are equal to $\sigma_{n}^{2}$. The $D$ eigenvectors $\mathbf{V}_{s}$ corresponding to the $D$ largest eigenvalues satisfy the relation

$$
\operatorname{Range}\left(\mathbf{V}_{s}\right)=\operatorname{Range}(\overline{\mathbf{A}}) \text {. }
$$

There exists a unique nonsingular $\mathbf{T}$ such that $\mathbf{V}_{s}=\overline{\mathbf{A}} \mathbf{T}$. Further, the invariance structure of the array allows the decomposition of $\mathbf{V}_{s}$ into $\mathbf{V}_{0} \in \mathbb{C}^{m \times D}$ and $\mathbf{V}_{1} \in$ $\mathbb{C}^{m \times D}$ such that $\mathbf{V}_{0}=\mathbf{A T}$ and $\mathbf{V}_{1}=\mathbf{A} \Phi \mathbf{T}$. This implies that

$$
\operatorname{Range}\left(\mathbf{V}_{0}\right)=\operatorname{Range}\left(\mathbf{V}_{1}\right)=\operatorname{Range}(\mathbf{A}) \text {. }
$$

Since $\mathbf{V}_{0}$ and $\mathbf{V}_{1}$ share a common column space, the rank of $\mathbf{V}_{01}=\left[\mathbf{V}_{0} \mid \mathbf{V}_{1}\right]$ is $D$. This implies that there exists a unique rank- $D$ matrix $\mathbf{F} \in \mathbb{C}^{2 D \times D}$, such that

$$
\left[\mathbf{V}_{0} \mid \mathbf{V}_{1}\right] \mathbf{F}=\mathbf{V}_{0} \mathbf{F}_{0}+\mathbf{V}_{1} \mathbf{F}_{1}=\mathbf{A T F}_{0}+\mathbf{A} \Phi \mathbf{T} \mathbf{F}_{1}=0
$$

where $\mathbf{F}$ spans the null space of $\mathbf{V}_{01}$. By defining $\Psi=-\mathbf{F}_{0} \mathbf{F}_{1}$, (A.30) can be rearranged to obtain

$$
\mathrm{AT} \Psi=\mathrm{A} \Phi \mathrm{T}
$$

which implies

$$
\mathrm{AT} \Psi \mathrm{T}^{-1}=\mathrm{A} \Phi .
$$

Assuming A to be full rank, which is true as long as the directions of arrivals of each signal is distinct, (A.32) states that

$$
\mathrm{T} \Psi=\Phi \mathrm{T} .
$$

From (A.33), the eigenvalues of $\boldsymbol{\Psi}$ must be equal to the diagonal elements of $\boldsymbol{\Phi}$, and the columns of $\mathbf{T}$ are the eigenvectors of $\mathbf{\Psi}$. The signal parameters are obtained as non-linear functions of the eigenvalues of the operator $\boldsymbol{\Psi}$, that maps (rotates) one set of vectors $\mathbf{V}_{0}$ that span an m-dimensional signal subspace into another set of 
vectors $\mathbf{V}_{1}$.

In many applications where only limited number of noisy measurements available, the condition in equation (A.28) and (A.29) is not satisfied. Hence, finding a $\boldsymbol{\Psi}$ such that $\hat{\mathbf{V}}_{0} \boldsymbol{\Psi}=\hat{\mathbf{V}}_{1}$ is not possible. Therefore, it is required to resort to a least squares solution which minimizes the residual error. Assuming that the set of equations is overdetermined the least squares solution is given by

$$
\boldsymbol{\Psi}=\left(\hat{\mathbf{V}}_{0}^{H} \hat{\mathbf{V}}_{0}\right)^{-1} \hat{\mathbf{V}}_{0}^{H} \hat{\mathbf{V}}_{1} .
$$

Once $\boldsymbol{\Psi}$ is obtained, its eigenvalues which correspond to the diagonal elements of $\boldsymbol{\Phi}$ can be easily computed. Since the diagonal elements of $\boldsymbol{\Phi}$ are related to the angle of arrival via (A.25), they can then be directly computed using

$$
\theta_{k}=\cos ^{-1}\left[c \frac{\arg \left(\Phi_{k}\right)}{\omega_{0} \Delta}\right] .
$$

Unlike other conventional DOA estimation techniques, ESPRIT eliminates the search procedure and produces the DOA estimates directly in terms of the eigenvalues. However, the MUSIC algorithm provides better accuracy and stability in comparison with ESPRIT [136].

\section{A.3 Compressive Sensing and Weighted Spatial Filter DOA Techniques}

This section provides mathematical background on $\ell_{2,1}$ DOA estimation and weighted spatial filter $\ell_{2,1}$ estimation techniques.

\section{A.3.1 Compressive Sensing $\ell_{2,1}$ DOA estimation}

Considering a ULA with $N$ sensors with half wavelength spacing between antenna elements. There are $L$ far-field narrowband source signals with $K$ snapshots, which 
can be written as [137]

$$
\mathbf{S}=\left[\begin{array}{ccc}
S\left(\theta_{1}, 1\right) & \cdots & S\left(\theta_{1}, K\right) \\
\vdots & \ddots & \vdots \\
S\left(\theta_{L}, 1\right) & & S\left(\theta_{L}, K\right)
\end{array}\right]
$$

where $\theta_{l}$ is the direction of the $l^{\text {th }}$ source signal. The received signal can be defined as

$$
\begin{aligned}
\mathbf{Z} & =[\mathbf{z}(1) \cdots \mathbf{z}(K)] \\
& =\left[\begin{array}{ccc}
z(1,1) & \cdots & z(1, K) \\
\vdots & \ddots & \vdots \\
z(N, 1) & & z(N, K)
\end{array}\right],
\end{aligned}
$$

where

$$
\mathbf{z}=\sum_{l=1}^{L} \mathbf{a}\left(\theta_{l}\right) \mathbf{s}\left(\theta_{l}, t\right)+\mathbf{n}(t)
$$

is the received signal at time $t$ and $\mathbf{a}\left(\theta_{l}\right)$ is the steering vector corresponding to the direction $\theta_{l}$ and $\mathbf{n}$ is the noise vector. In the context of radar, the steering vector $\mathbf{a}\left(\theta_{l}\right)$ depends on the angle of arrival $\theta_{l}$. Hence, the DOA estimation problem can be defined as finding the unknown directions $\theta_{l}$ given $\mathbf{z}(t)$ and the mapping $\theta_{l} \mapsto \mathbf{a}\left(\theta_{l}\right)$ for any $l$. However, $\mathbf{a}\left(\theta_{l}\right)$ is still unknown since it is determined by $\theta_{l}$.

Using the CS framework with over-complete representation of $\mathbf{Z}$ which divides the search range into many DOAs of potential source locations and each potential DOA corresponds to a grid. In general, the number of potential directions $P$ is much larger than the number of the true sources $L$ and the number of the sensors $N$. The steering matrix with all potential DOAs can written as

$$
\mathbf{A}=\left[\mathbf{a}\left(\theta_{1}\right), \ldots, \mathbf{a}\left(\theta_{P}\right)\right]
$$

Therefore, the received signal $\mathbf{z}(t)$ can be formulated as the sparse representation 
problem

$$
\mathbf{z}(t)=\sum_{p=1}^{P} \mathbf{a}\left(\theta_{p}\right) \mathbf{x}\left(\theta_{p}, t\right)+\mathbf{n}(t)
$$

where $\mathbf{x}\left(\theta_{p}, t\right)$ is the source signal from $\theta_{p}$ at time $t$. The matrix form can be defined as

$$
\mathrm{Z}=\mathbf{A X}+\mathbf{N}
$$

where

$$
\mathbf{X}=\left[\begin{array}{ccc}
x\left(\theta_{1}, 1\right) & \cdots & x\left(\theta_{1}, K\right) \\
\vdots & \ddots & \vdots \\
x\left(\theta_{P}, 1\right) & \cdots & x\left(\theta_{P}, K\right)
\end{array}\right]
$$

The true direction can be treated as time-invariant during the period of observation. If $\theta_{p}=\theta_{l}$ for some $l$, the elements in $p^{t h}$ row of $\mathbf{X}$ is non-zero and equal to the elements in $k^{\text {th }}$ row of $\mathbf{S}$, otherwise all elements in the rows are zeros. Hence, if $\mathbf{X}$ is solved by (A.42), the $\theta_{p}$ corresponding to the non-zero rows in $\mathbf{X}$ leads to the DOA estimation. It can be found that only $L$ rows of $\mathbf{X}$ are non-zero, and such an $\mathbf{X}$ is called jointly sparse [9]. The CS-based DOA estimation can be modified to solve the jointly sparse $\mathrm{X}$ by solving the following optimization problem

$$
\begin{array}{ll}
\min & \|\mathbf{X}\|_{2,1} \\
\text { subject to } & \|\mathbf{Y}-\mathbf{A X}\|_{F} \leqslant \epsilon,
\end{array}
$$

where $\epsilon$ is a small constant to limit the residual error and $\|\mathbf{X}\|_{2,1}=\sum_{p} \sqrt{\sum_{t} x^{2}\left(\theta_{p}, t\right)}$. The $\epsilon$ can be bounded by $\epsilon \geq \mathbf{N}$. The regular $\ell_{2,1}$-minimization term ensures the number of the non-zero rows in $\mathbf{X}$ be least, while $\ell_{F}$-minimization term ensures the error be least. Once the optimization problem converges, the peaks of $\ell_{2}$-norm of each row in $\mathbf{X}$ are the estimated DOAs [104]. 


\section{A.3.2 Weighted Spatial Filter $\ell_{2,1}$ DOA Estimation}

In this section, the spatial filter is used to design a weighting matrix to improve the performance by adjusting the sparse constraint. In order to construct the spatial filter, the received signals are divided into two parts: the reference signals which are received by the first sensor and the input signals which are received by other sensors which are expressed as [104]

$$
\mathbf{Z}=\left[\begin{array}{l}
\mathbf{Z}_{1} \\
\mathbf{Z}_{2}
\end{array}\right]
$$

where

$$
\mathbf{Z}_{1}=[z(1,1), \cdots, z(1, K)]
$$

is the reference signal and

$$
\mathbf{Z}_{2}=\left[\begin{array}{ccc}
z(2,1) & \cdots & z(2, K) \\
\vdots & \ddots & \vdots \\
z(N, 1) & \cdots & z(N, K)
\end{array}\right]
$$

is the input signal. According to [18], the parameters in the spatial filter $\mathbf{w}^{H}=$ $\left[w_{1}, \ldots, w_{N-1}\right]$ can be found to make the output signals $u(t)$ of the spatial filter be equal to the reference signals $y(1, t)$

$$
e(t)=u(t)-y(1, t)=\mathbf{w}^{H} \mathbf{Z}_{2}(t)-z(1, t)
$$

where $\mathbf{Z}_{2}(t)$ is the $t$ th column in $\mathbf{Z}_{2}$. The least-square (LS) cost function is used to find the optimal parameters $\mathbf{w}$ which can be defined as

$$
\mathbf{J}(w)=\left|\sum_{t=1}^{K} e(t)\right|^{2}=\left|\sum_{t=1}^{K} \mathbf{w}^{H} \mathbf{Z}_{2}(t)-z(1, t)\right|^{2}
$$


The gradient of (A.49) is:

$$
\nabla \mathbf{J}(w)=2 \sum_{i=1}^{K} \sum_{j=1}^{K} \mathbf{Z}_{2}(i) \mathbf{Z}_{2}^{H}(j) \mathbf{w}-2 \sum_{i=1}^{K} \sum_{j=1}^{K} \mathbf{Z}_{2}(i) z^{*}(1, j) .
$$

Setting $\nabla \mathbf{J}(w)=0$, the spatial filter parameters can be determined

$$
\mathbf{w}^{H}=\mathbf{Z}_{1} \mathbf{Z}_{2}^{H}\left[\mathbf{Z}_{2} \mathbf{Z}_{2}^{H}\right]^{-1} .
$$

Hence, the received signals can be eliminated by

$$
\left[\begin{array}{ll}
-1 & \mathbf{w}^{H}
\end{array}\right]\left[\begin{array}{l}
\mathbf{Z}_{1} \\
\mathbf{Z}_{2}
\end{array}\right]=-\mathbf{Z}_{1}+\mathbf{w}^{H} \mathbf{Z}_{2}=0,
$$

where 0 denotes a null matrix of suitable dimension. (A.52) can be simplified to

$$
\mathbf{w}_{c}^{H} \mathbf{Z}=0
$$

where

$$
\mathbf{w}_{c}^{H}=\left[\begin{array}{ll}
-1 & \mathbf{w}^{H}
\end{array}\right]
$$

is the spatial filter which can eliminate the signals from the directions $\theta_{k}$. Thus, the space spectrum of the spatial filter can be achieved by

$$
Q\left(\theta_{p}\right)=\left|\mathbf{w}_{c}^{H} \mathbf{a}\left(\theta_{p}\right)\right|
$$

The space spectrum $Q\left(\theta_{p}\right)$ is close to 0 when $\theta_{p}$ is close to $\theta_{l}$. Consider only one signal from direction $\theta_{k}$ coming from a noisy environment. Thus, (A.53) can be rewritten as

$$
\mathbf{w}_{c}^{H} \mathbf{Z}=\mathbf{w}_{c}^{H}\left[\mathbf{a}\left(\theta_{p}\right) \mathbf{X}+\mathbf{N}\right]=0 .
$$

It yields that

$$
\mathbf{w}_{c}^{H}\left[\mathbf{a}\left(\theta_{p}\right)+\mathbf{N X}^{+}\right]=0
$$

where $(.)^{+}$denotes the pseudo-inverse of a matrix. The accuracy of $\mathbf{Q}\left(\theta_{p}\right)$ in (A.57) 
depends on two main factors: the power of $\mathbf{N}$ and the correlation between $\mathbf{N}$ and $\mathbf{X}^{+}$. The power of $\mathbf{N}$ is reflected in the signal to noise ratio (SNR) and the correlation between $\mathbf{N}$ and $\mathbf{X}^{+}$can be reflected in the number of data (i.e., snapshots and sensors). The directions of the source signals are nulled by the spatial filter. Therefore, the weighting matrix can be written as

$$
\mathbf{Q}=\left[\begin{array}{ccc}
Q\left(\theta_{1}\right) / Q_{\max } & & 0 \\
& \ddots & \\
0 & & Q\left(\theta_{P}\right) / Q_{\max }
\end{array}\right]
$$

where $\mathbf{Q}_{\max }$ is the max value of $\mathbf{Q}\left(\theta_{p}\right)$. Thus, the weighted $\ell_{2,1}$-minimization problem can stated as

$$
\begin{array}{ll}
\min & \|\mathbf{Q X}\|_{2,1} \\
\text { subject to } & \|\mathbf{Y}-\mathbf{A X}\|_{F} \leqslant \epsilon,
\end{array}
$$

The WSF CS-based DOA estimation provides higher accuracy than the regular $\ell_{2,1^{-}}$ minimization since the weighting matrix $\mathbf{Q}$ can adjust the sparse penalty for each element in $\mathbf{X}$. WSF matrix does not require additional parameter settings in comparison with $\ell_{2,1}$-minimization, however it adds only one step to calculate the weighting matrix. This step mainly consists of solving the pseudo-inverse in (A.51). 


\section{Appendix B}

\section{Interference plus Noise Covariance matrix Estimation}

This Appendix provides a brief description of the interference-plus-noise covariance matrix estimation techniques discussed in this thesis.

\section{B.1 Sample Covariance Matrix with Adaptive Di- agonal Loading}

The sample covariance matrix can be defined as

$$
\mathbf{R}_{s c m}=\frac{1}{K} \sum_{k=1}^{K} \mathbf{y}(k) \mathbf{y}^{H}(k)
$$

where $K$ is the number of received snapshots. However, the interference-plus-noise covariance matrix can be defined in term of $\mathbf{R}_{s c m}$ and inaccuracy factor [114]

$$
\widehat{\mathbf{R}}_{j+n}=\mathbf{R}_{s c m}+\varepsilon \mathbf{B}
$$

where $\varepsilon \mathbf{B}$ is an inaccuracy random matrix with zero mean and unit variance and all its

elements are $\gg 1$. The inaccuracy of $\widehat{\mathbf{R}}_{j+n}$ can be rectified by adding a constant value to its diagonal elements. The diagonally loaded interference-plus-noise covariance matrix $\widetilde{\mathbf{R}}_{j+n}$ can be expressed as

$$
\widetilde{\mathbf{R}}_{j+n}=\mathbf{R}_{s c m}+\varepsilon \mathbf{B}+\iota \mathbf{I}
$$


where $\iota$ is the diagonal loading factor.

Adaptive diagonal loading can change the diagonal loading value according to the received snapshot, which decreases the interval of diagonal loading value depending on the relation between the inaccuracy of covariance matrix and the diagonal loading value. Using matrix identity, the diagonally loaded interference-plus-noise covariance matrix $\widetilde{\mathbf{R}}_{j+n}$ can be written as

$$
\mathbf{R}_{s c m}+\varepsilon \mathbf{B}+\iota \mathbf{I}=\left(\mathbf{R}_{s c m}+\iota \mathbf{I}\right)\left[\mathbf{I}+\varepsilon \mathbf{B}\left(\mathbf{R}_{s c m}+\iota \mathbf{I}\right)^{-1}\right]
$$

The inverse of $\tilde{\mathbf{R}}_{j+n}$ can be expressed as

$$
\widetilde{\mathbf{R}}_{j+n}^{-1}=\left[\mathbf{I}+\varepsilon \mathbf{B}\left(\mathbf{R}_{s c m}+\iota \mathbf{I}\right)^{-1}\right]^{-1}\left(\mathbf{R}_{s c m}+\iota \mathbf{I}\right)^{-1} .
$$

The $\left[\mathbf{I}+\varepsilon \mathbf{B}\left(\mathbf{R}_{s c m}+\iota \mathbf{I}\right)^{-1}\right]$ can be unfolded using the matrix identity $(\mathbf{I}+\mathbf{A})(\mathbf{I}-\mathbf{A})=$ $\mathbf{I}-\mathbf{A}^{2}$ and written as

$$
\left[\mathbf{I}+\varepsilon \mathbf{B}\left(\mathbf{R}_{s c m}+\iota \mathbf{I}\right)^{-1}\right]\left[\mathbf{I}-\varepsilon \mathbf{B}\left(\mathbf{R}_{s c m}+\iota \mathbf{I}\right)^{-1}\right]=\mathbf{I}-\left[\varepsilon \mathbf{B}\left(\mathbf{R}_{s c m}+\iota \mathbf{I}\right)^{-1}\right]^{2} .
$$

Since the $\varepsilon \mathbf{B}$ is very small in comparison with $\mathbf{R}_{s c m}+\iota \mathbf{I}$, hence (B.6) is reduce to $\left[\mathbf{I}+\varepsilon \mathbf{B}\left(\mathbf{R}_{s c m}+\iota \mathbf{I}\right)^{-1}\right]\left[\mathbf{I}-\varepsilon \mathbf{B}\left(\mathbf{R}_{s c m}+\iota \mathbf{I}\right)^{-1}\right] \approx \mathbf{I}$ and $\left[\mathbf{I}+\varepsilon \mathbf{B}\left(\mathbf{R}_{s c m}+\iota \mathbf{I}\right)^{-1}\right]=$ $\left[\mathbf{I}-\varepsilon \mathbf{B}\left(\mathbf{R}_{s c m}+\iota \mathbf{I}\right)^{-1}\right]^{-1}$, so taking the inverse, we have

$$
\left[\mathbf{I}+\varepsilon \mathbf{B}\left(\mathbf{R}_{s c m}+\iota \mathbf{I}\right)^{-1}\right]^{-1}=\mathbf{I}-\varepsilon \mathbf{B}\left(\mathbf{R}_{s c m}+\iota \mathbf{I}\right)^{-1} .
$$

The interference-plus-noise in (B.5) can be rewritten as

$$
\widetilde{\mathbf{R}}_{j+n}^{-1}=\varepsilon \mathbf{B}\left(\mathbf{R}_{s c m}+\iota \mathbf{I}\right)^{-1}\left[\mathbf{I}-\varepsilon \mathbf{B}\left(\mathbf{R}_{s c m}+\iota \mathbf{I}\right)^{-1}\right] .
$$

The sample covariance matrix without the desired signal can be expressed in terms of interference-plus-noise as

$$
\mathbf{R}_{s c m}=\sum_{j=1}^{J} \sigma_{j}^{2} \mathbf{s}\left(\theta_{j}\right) \mathbf{s}^{H}\left(\theta_{j}\right)+\sigma_{n}=\mathbf{S}_{j} \boldsymbol{\Omega}_{j} \mathbf{S}_{j}^{H}
$$

where $\sigma_{j}^{2}$ is the interference signal power corresponding to the $k^{\text {th }}$ interference, $\sigma_{n}^{2}$ is the noise power, $J$ is the number of interference signals and $\Omega_{j}=\left[\sigma_{1}^{2}, \ldots, \sigma_{J}^{2}\right]$ is a diagonal matrix associated with the interference signal power. Using the matrix 
inversion lemma [138], and substituting (B.9) into (B.8), we have [114]

$$
\widetilde{\mathbf{R}}_{j+n}^{-1}=\left(\mathbf{R}_{s c m}+\iota \mathbf{I}\right)^{-1}\left\{\mathbf{I}-\frac{\varepsilon}{\iota+\sigma_{n}^{2}} \mathbf{B C}\right\}
$$

where

$$
\mathbf{C}=\left[\mathbf{I}-\mathbf{S}\left(\mathbf{S}^{H} \mathbf{S}+\left(\sigma_{n}^{2}+\iota\right) \Omega^{-1} \mathbf{S}^{-1}\right)\right]
$$

The diagonal loading factor $\iota$ should be very small in comparison with the diagonal elements of the sample covariance matrix $\mathbf{R}_{s c m}$. From (B.10), the degradation factor of the MVDR beamforming is directly proportional to $\varepsilon /\left(\iota+\sigma_{n}^{2}\right) \mathbf{B C}$. By setting $\mathbf{I}-\varepsilon /\left(\iota+\sigma_{n}^{2}\right) \mathbf{B C} \approx \mathbf{I}$, hence the diagonal loading factor can be controlled by

$$
\varepsilon-\sigma_{n}^{2} \leqslant \iota \ll \mathbf{R}_{s c m}(i, i) .
$$

The inaccuracy factor $\varepsilon$ of the sample covariance matrix can be obtained by the standard deviation of the diagonal elements of the sample covariance matrix, $\varepsilon=$ $\operatorname{std}\left(\operatorname{diag}\left(\mathbf{R}_{s c m}\right)\right)$. The MVDR beamforming optimization problem with an adaptive diagonally loaded interference-plus-noise covariance matrix $\widetilde{\mathbf{R}}_{j+n}$ can written as

$$
\begin{array}{ll}
\min _{\boldsymbol{\omega}} & \boldsymbol{\omega}^{H} \widetilde{\mathbf{R}}_{j+n} \boldsymbol{\omega} \\
\text { s.t. } & \boldsymbol{\omega}^{H} \mathbf{a}=1 .
\end{array}
$$

And the optimal solution of the MVDR optimization problem is expressed as

$$
\boldsymbol{\omega}=\frac{\widetilde{\mathbf{R}}_{j+n}^{-1} \mathbf{a}}{\mathbf{a}^{H} \widetilde{\mathbf{R}}_{j+n}^{-1} \mathbf{a}} .
$$

\section{B.2 Shrinkage Algorithm for Covariance Matrix Estimation}

Let $\left\{\mathbf{x}_{i}\right\}_{i=1}^{n}$ be a sample of independent identical distributed (i.i.d.) $p$-dimensional Gaussian vectors with zero mean and covariance $\mathbf{R}_{x}$. The objective is to find an 
estimator $\widehat{\mathbf{R}}_{x}\left(\left\{\mathbf{x}_{i}\right\}_{i=1}^{n}\right)$ which minimizes the mean square error (MSE)

$$
\mathbb{E}\left\{\left\|\widehat{\mathbf{R}}_{x}-\mathbf{R}_{x}\right\|_{F}^{2}\right\}
$$

It is difficult to compute the mean squared error (MSE) of $\hat{\mathbf{R}}_{x}$ without additional constraints, so shrinkage $[139,140]$ can be employed as a specific class estimator. The unstructured classical estimator of $\mathbf{R}_{x}$ is the sample covariance matrix shown in (5.10). The estimator is unbiased $\mathbb{E}\left\{\mathbf{R}_{S C M}\right\}$ and it is also the maximum likelihood estimation if $n \geq p$. We consider a naive but well-conditioned estimate for $\mathbf{R}_{x}$

$$
\widehat{\mathbf{F}}=\frac{\operatorname{tr}\left(\mathbf{R}_{S C M}\right)}{p} \mathbf{I}
$$

This structured estimate will lead to a lower variance at the cost of increasing the bias. A trade-off between low bias and low variance is achieved by shrinkage of $\mathbf{R}_{S C M}$ toward $\widehat{\mathbf{F}}$, which can be defined in the following estimator

$$
\widehat{\mathbf{R}}_{x}=(1-\hat{\rho}) \mathbf{R}_{S C M}+\hat{\rho} \widehat{\mathbf{F}}
$$

The estimator $\widehat{\mathbf{R}}_{x}$ is characterized by the shrinkage coefficient $\hat{\rho}$, which is a parameter between 0 and 1 and can be a function of the snapshots $\left\{\mathbf{x}_{i}\right\}_{i=1}^{n}$. The $\widehat{\mathbf{F}}$ is referred to as shrinkage target.

The objective is to find a shrinkage coefficient $\hat{\rho}$ that minimizes the MSE in (B.15). The optimal $\hat{\rho}$ minimizing the MSE depends in general on the unknown covariance $\mathbf{R}_{x}$ and therefore we have to employ Oracle estimator [141] and Ledoit-Wolf estimators to approximate the optimal shrinkage coefficient.

Clairvoyant oracle estimator [141], which exploits the optimal non-random coefficient to minimize the mean-squared error can be defined as

$$
\begin{array}{lr}
\min _{\hat{\rho}} & \mathbb{E}\left\{\left\|\widehat{\mathbf{R}}_{O}-\mathbf{R}_{x}\right\|_{F}^{2}\right\} \\
\text { s.t. } & \widehat{\mathbf{R}}_{O}=(1-\hat{\rho}) \mathbf{R}_{S C M}+\hat{\rho} \widehat{\mathbf{F}}
\end{array}
$$

where $\widehat{\mathbf{R}}_{O}$ is the Oracle estimator. The solution for this optimization problem is provided in the following theorem.

Theorem B.2.1 [141] let $\mathbf{R}_{S C M}$ be the sample convariance of a set of $p$-dimensional 
vectors $\left\{\mathbf{x}_{i}\right\}_{i=1}^{n}$. If $\left\{\mathbf{x}_{i}\right\}_{i=1}^{n}$ are i.i.d Gaussian vectors with covariance matrix $\mathbf{R}_{x}$, then the solution to (B.18) is

$$
\begin{aligned}
\hat{\rho}_{O} & =\frac{\operatorname{tr}\left(\left(\mathbf{R}_{x}-\mathbf{R}_{S C M}\right)\left(\widehat{\mathbf{F}}-\mathbf{R}_{S C M}\right)\right)}{\mathbb{E}\left\{\left\|\mathbf{R}_{S C M}-\widehat{\mathbf{F}}\right\|_{F}^{2}\right\}} \\
& =\frac{(1-2 / p) \operatorname{tr}\left(\mathbf{R}_{x}^{2}\right)+t r^{2}\left(\mathbf{R}_{x}\right)}{(n+1-2 / p) \operatorname{tr}\left(\mathbf{R}_{x}^{2}\right)+(1-n / p) \operatorname{tr}^{2}\left(\mathbf{R}_{x}\right)} .
\end{aligned}
$$

The proof of this theorem is established in [115]. (B.20) holds for Gaussian distribution. The Oracle estimator defined by (B.18) is optimal but cannot be implemented since the solution specified depends on unknown $\mathbf{R}_{x}$. Without any knowledge of the sample distribution, LW approximated the oracle using the following consistent estimate of (B.19)

$$
\hat{\rho}_{L W}=\frac{\sum_{i=1}^{n}\left\|\mathbf{x}_{i} \mathbf{x}_{i}^{T}-\mathbf{R}_{S C M}\right\|_{F}^{2}}{n^{2}\left[\operatorname{tr}\left(\mathbf{R}_{S C M}^{2}\right)-\operatorname{tr}^{2}\left(\mathbf{R}_{S C M}\right) / p\right]} .
$$

The LW estimator $\widehat{\mathbf{R}}_{L W}$ is then defined by plugging the $\hat{\rho}_{L W}$ into (B.17) we get

$$
\widehat{\mathbf{R}}_{L W}=\left(1-\hat{\rho}_{L W}\right) \mathbf{R}_{S C M}+\hat{\rho}_{L W} \widehat{\mathbf{F}} .
$$

The value of $\rho_{L W}$ always lies between 0 and 1 .

\section{B.3 Subspace Based Interference-plus-Noise Co- variance Matrix Estimation}

The eigen-decomposition of the theoretical data covariance matrix can be split into two covariance matrices as

$$
\mathbf{R}_{S C M}=\mathbf{R}_{z}+\mathbf{R}_{j+n}
$$

where $\mathbf{R}_{z}$ is the covariance for the signal only. Further decomposing (B.23) yields

$$
\mathbf{R}_{S C M}=\mathbf{V} \boldsymbol{\Lambda} \mathbf{V}=\mathbf{V}_{z} \boldsymbol{\Lambda}_{z} \mathbf{V}_{z}^{H}+\mathbf{V}_{j+n} \Lambda_{j+n} \mathbf{V}_{j+n}^{H}
$$


where the unitary matrix $\mathbf{V}=\left[\mathbf{v}_{1}, \mathbf{v}_{2}, \ldots, \mathbf{v}_{n}\right]$ contians all the eigenvectors, and the diagonal matrix $\boldsymbol{\Lambda}=\operatorname{diag}\left[\lambda_{1}, \lambda_{2}, \ldots, \lambda_{n}\right]$ contains the corresponding eigenvalues. The eigen-decomposition of (B.24) can be written as the expanded form of the covariance matrix

$$
\mathbf{R}_{S C M}=\sigma_{z}^{2} \mathbf{s}_{z} \mathbf{s}_{z}^{H}+\sum_{j=1}^{J} \sigma_{j}^{2} \mathbf{s}_{j} \mathbf{s}_{j}^{H}+\sigma_{n}^{2} \mathbf{I} .
$$

Therefore, the interference-plus-noise covariance matrix $\mathbf{R}_{j+n}$ can be estimated by removing the desired signal component from the sample covariance matrix using

$$
\widehat{\mathbf{R}}_{j+n}=\mathbf{R}_{S C M}-\hat{\lambda}_{z} \hat{\mathbf{v}}_{z} \hat{\mathbf{v}}_{z}^{H}
$$

where $\hat{\lambda}_{z}$ and $\hat{\mathbf{v}}_{z}$ are the eigenvalue and eigenvector of the sample covariance matrix corresponding to the desired signal waveform $z(k)$ impinging from a specific angular sector.

Using Capon spatial spectrum, we can identify the desired signal eigenvector by reconstructing the signal covariance matrix as [90]

$$
\widetilde{\mathbf{R}}_{z}=\int_{\Theta} \hat{p}(\theta) \mathbf{d}(\theta) \mathbf{d}^{H}(\theta) d \theta
$$

where

$$
\hat{p}(\theta)=\frac{1}{\mathbf{d}(\theta) \mathbf{R}_{S C M}^{-1} \mathbf{d}^{H}(\theta)}
$$

is the Capon spatial spectrum estimator and the angular sector $\Theta$ contains the desired signal direction $\theta_{z}$ only while DOAs of the interferers do not belong to the angular sector $\Theta$ and $\mathbf{d}(\theta)$ is the steering vector associated with angle $\theta$. $\widetilde{\mathbf{R}}_{z}$ collects the information of the desired signal and noise in the angular sector and excludes the effect of the interference.

The signal subspace of the reconstructed signal covariance matrix $\widetilde{\mathbf{R}}_{z}$ is spanned by the columns of the column orthogonal matrix $\mathbf{V}_{z}$. The actual steering vector of the desired signal belong to the signal subspace of $\widetilde{\mathbf{R}}_{z}$ spanned by $\mathbf{V}_{z}$. Also, the signal steering vector belongs to the signal subspace $\mathbf{P}_{z}=\mathbf{V}_{z} \mathbf{V}_{z}^{H}$ and is orthogonal to the projection subspace $\mathbf{P}_{z}^{\perp}=\mathbf{I}-\mathbf{V}_{z} \mathbf{V}_{z}^{H}$, hence the eigenvector associated with 
the desired signal can be estimated according to

$$
\hat{\mathbf{v}}_{z}=\underset{\mathbf{v} \in \mathbf{V}}{\operatorname{argmax}} \frac{\left\|\mathbf{P}_{z} \mathbf{v}\right\|}{\left\|\mathbf{P}_{z}^{\perp} \mathbf{v}\right\|}=\underset{\mathbf{v} \in \mathbf{V}}{\operatorname{argmax}} \frac{\left\|\mathbf{V}_{z} \mathbf{V}_{z}^{H} \mathbf{v}\right\|}{\left\|\mathbf{I}-\mathbf{V}_{z} \mathbf{V}_{z}^{H} \mathbf{v}\right\|} .
$$

Substituting $\hat{\mathbf{v}}_{z}$ into (B.26), the estimated interference-plus-noise covariance matrix $\widehat{\mathbf{R}}_{j+n}$ can be estimated with the desired signal component effectively removed from the sample covariance matrix. Avoiding rank deficiency, $\widehat{\mathbf{R}}_{j+n}$ is diagonal loading with the smallest eigenvalue of the sample covariance matrix $\lambda_{\min }$.

The adaptive MVDR beamforming weights in (5.9) can be rewritten with the subspace based estimated interference-plus-noise covariance matrix as [142]

$$
\boldsymbol{\omega}=\frac{\left[\widetilde{\mathbf{R}}_{j+n}+\lambda_{\min } \mathbf{I}\right]^{-1} \hat{\mathbf{v}}_{z}}{\hat{\mathbf{v}}_{z}^{H}\left[\widetilde{\mathbf{R}}_{j+n}+\lambda_{\min } \mathbf{I}\right]^{-1} \hat{\mathbf{v}}_{z}}
$$

where the signal steering vector $\mathbf{v}$ is replaced with estimated signal eigenvector $\hat{\mathbf{v}}_{z}$.

\section{B.4 Sparsity-Based Interference-plus-Noise Co- variance Matrix Estimation}

From (B.25), the interference-plus-noise covariance matrix has the form of

$$
\mathbf{R}_{j+n}=\sum_{j=1}^{J} \sigma_{j}^{2} \mathbf{s}_{j} \mathbf{s}_{j}^{H}+\sigma_{n}^{2} \mathbf{I}
$$

where $J$ is the number of interferers, $\mathbf{s}_{j}$ is the steering vector of the $j$ th interference impinging on the antenna array and $\sigma_{j}^{2}$ is the corresponding interference power. The sparsity condition is based on the assumption that the number of array sensors is typically larger than the number of sources impinging on the array.

According to (B.31), the interference-plus-noise covariance matrix is a function of the directions and power of interferers, as well as the noise power. The sparsityconstrained covariance matrix estimation problem can be formulated according to 
(B.25) as

$$
\begin{array}{ll}
\min _{p, \sigma_{n}^{2}} & \left\|\mathbf{R}_{S C M}-\mathbf{S P S}^{H}-\sigma_{n}^{2} \mathbf{I}\right\|_{F} \\
\text { s.t. } & \|\mathbf{p}\|_{0}=J+1 \\
& \mathbf{p} \geqslant 0 \\
& \sigma_{n}^{2}>0
\end{array}
$$

where $\mathbf{p} \in \mathbb{R}_{+}^{M}$ is the spatial spectrum distribution on the sample grid of the observed spatial domain (e.g., $\left.\left\{\theta_{1}, \theta_{2}, \ldots, \theta_{M}\right\} \in \Theta\right), \mathbf{p}=\operatorname{diag}\left(\mathbf{p} \in \mathbb{R}_{+}^{M \times M}\right)$ is the corresponding diagonal matrix, $\mathbf{S}$ is the array manifold matrix and $\|\cdot\|_{0}$ is the $\ell_{0}$ norm of a vector. The main goal of (B.32) is to find the sparsest spatial spectrum distribution $\mathbf{p}$ and the noise power $\sigma_{n}^{2}$ such that the difference between the resulting covariance matrix $\mathbf{S P S}^{H}-\sigma_{n}^{2} \mathbf{I}$ and the sample covariance matrix $\mathbf{R}_{S C M}$ is minimized. The non-convex nature of $\ell_{0}$-norm in (B.32) makes it a difficult combinatorial optimization problem [143]. By relaxing the $\ell_{0}$-norm and approximately replace it by $\ell_{1}$-norm, (B.32) can be formulates as a convex optimization problem

$$
\begin{array}{ll}
\min _{\boldsymbol{p}, \sigma_{n}^{2}} & \left\|\mathbf{R}_{S C M}-\mathbf{S P S}^{H}-\sigma_{n}^{2} \mathbf{I}\right\|_{F} \\
\text { s.t. } & \|\mathbf{p}\|_{0} \leqslant \sigma_{z}^{2}+\sum_{k=1}^{K} \sigma_{k}^{2}+\sigma_{n}^{2}+\eta \\
& \mathbf{p} \geqslant 0 \\
& \sigma_{n}^{2}>0
\end{array}
$$

where $\ell_{1}$-norm of $\mathbf{p}$ equals the total power of all sources (e.g., $\left.\sigma_{z}^{2}+\sum_{k=1}^{K} \sigma_{k}^{2}+\sigma_{n}^{2}+\eta\right)$, and a small number $\eta>0$ is added to the power constraint to allow the optimization problem to search for $\mathbf{p}$. This convex optimization problem can be reformulated as a basis pursuit de-noising (BPDN) problem [123] as

$$
\begin{aligned}
\min _{\boldsymbol{p}, \sigma_{n}^{2}} & \left\|\mathbf{R}_{S C M}-\mathbf{S P S}^{H}-\sigma_{n}^{2} \mathbf{I}\right\|_{F}+\varsigma\|\mathbf{p}\|_{1} \\
\text { s.t. } & \mathbf{p} \geqslant 0
\end{aligned}
$$

where $\varsigma$ is a regularization parameter controlling the trade-off between the sparsity of the spatial spectrum and the residual norm of covariance matrix fitting. The optimization problem is convex and can be solved using standard and highly efficient interior point methods. However, the solution of this optimization problem is not 
absolutely sparse because of the $\ell_{1}$-norm relaxation. In addition, the determination of the regularization parameter $\varsigma$ can be challenging in some scenarios [117]. 